\title{
Rh(III)-Catalyzed Annulation of 2-Biphenylboronic Acid with Diverse Activated Alkenes
}

\author{
Bingxian Liu ${ }^{1 *}$, Lingyun Yang $^{1}$, Zhenzhen Dong $^{1}$, Junbiao Chang $^{1 *}$, Xingwei $\mathrm{Li}^{1,2^{*}}$ \\ ${ }^{1}$ NMPA Key Laboratory for Research and Evaluation of Innovative Drug, Collaborative \\ Innovation Center of Henan Province forGreen Manufacturing of Fine Chemicals, School \\ of Chemistry and Chemical Engineering, Henan Normal University,Xinxiang, \\ Henan453007, China \\ ${ }^{2}$ School of Chemistry and Chemical Engineering, Shaanxi Normal University (SNNU), \\ $X i$ 'an 710062, China
}

\section{Table of Contents}

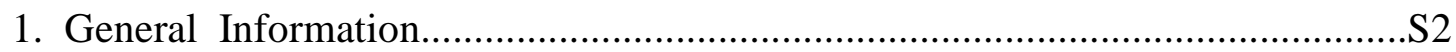

2. General Procedure for the Preparation of Substrates..........................S2

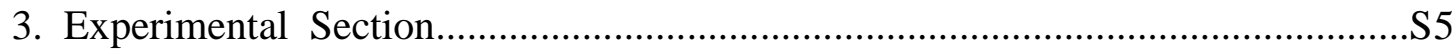

4. Possible mechnisms for formation of 3wa and 5.........................S12

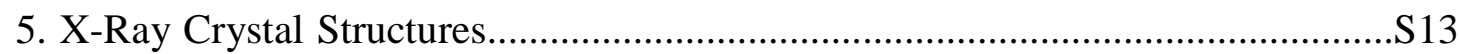

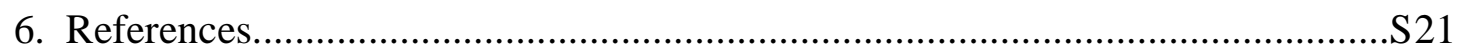

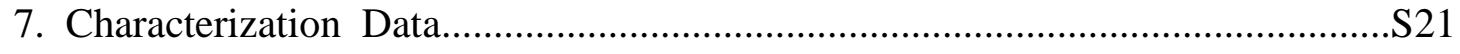

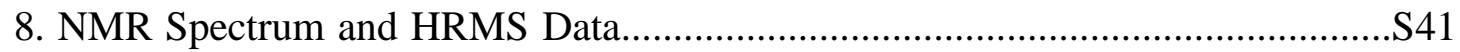




\section{General Information}

All the reactions were carried out under air atmosphere unless otherwise mentioned. The ${ }^{1} \mathrm{H}$ NMR spectra were recorded on a $400 \mathrm{MHz}$ or $600 \mathrm{MHz}$ NMR spectrometer. The ${ }^{13} \mathrm{C}$ NMR spectra were recorded at $100 \mathrm{MHz}$ or $150 \mathrm{MHz}$. The ${ }^{19} \mathrm{~F}$ NMR spectra were recorded at 376 or $565 \mathrm{MHz}$. The residual solvent signals were used as references and the chemical shifts were converted to the TMS scale $\left(\mathrm{CDCl}_{3}: \delta \mathrm{H}=7.26 \mathrm{ppm}, \delta \mathrm{C}=77.16 \mathrm{ppm}\right)$. The coupling constants were given in Hz. HRMS data were obtained using ESI-TOF mode. Single crystal XRD analysis of 3ae, 3af, 3-I, 5ab were recorded on a SuperNova Dual diffractometer using graphitemonochromated Co K $\alpha$ radiation, $\lambda=$ $0.71073 \AA$ and Single crystal XRD analysis of 3wa and $\mathbf{1 1}$ were recorded on a SuperNova Dual diffractometer using graphitemonochromated $\mathrm{Cu} \mathrm{K} \alpha$ radiation, $\lambda=1.54184 \AA$. All single crystals were prepared in DCM / $\mathrm{n}$-hexane (dissolve the compound in DCM and place in a penicillin bottle. Slowly dribble n-hexane into the bottle so that DCM and n-hexane form discreet layers). The conversion of starting materials was monitored by thin layer chromatography (TLC) using silica gel plates (silica gel 60 F254 $0.25 \mathrm{~mm}$ ), and components were visualized under UV light (254 and $365 \mathrm{~nm}$ ). Column chromatography was performed on silica gel 200-300 mesh. Reactions were heated by metal sand bath. (WATTCAS, Z100500). Unless otherwise noted, all other compounds have been reported in the literature or are commercially available. Commercial reagents were used without further purification. Arylboronic acids ${ }^{1}, \alpha, \beta$-unsaturated ketones ${ }^{2}$, cyclopropenones ${ }^{3}$, catalyst ${ }^{4}\left[\mathrm{Cp}^{\mathrm{tBu}} \mathrm{RhI}_{2}\right]_{2}$ were prepared according to published procedures.

\section{General Procedures for the Preparation of Substrates}

(a) Synthesis of 2-Bromobiphenyls ${ }^{1}$

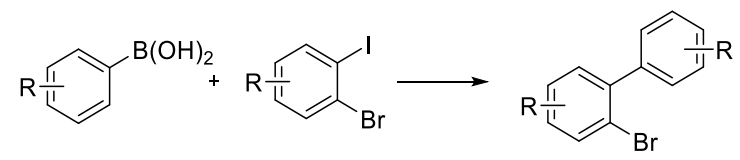

Following a reported procedure, a flask equipped with a reflux condenser is charged with the corresponding iodobenzene derivative $(1.00-1.13 \mathrm{eq})$, the corresponding boronic acid (1.00 - $1.11 \mathrm{eq})$, potassium carbonate $(1.14-1.38 \mathrm{eq})$, and tetrakis(triphenylphosphine)- palladium(0) $(1.99-2.13$ $\mathrm{mol} \%)$. The reactants are dissolved in a degassed mixture of benzene/ethanol/water $(0.125 \mathrm{M}, \mathrm{v}: \mathrm{v}: \mathrm{v}=$ 15:7:2), and the reaction mixture is stirred at $100{ }^{\circ} \mathrm{C}$ for $3-27 \mathrm{~h}$. After cooling to room temperature, water is added, and the aqueous phase is extracted with diethyl ether. The combined organic phases are dried over $\mathrm{MgSO}_{4}$, and the solvents are evaporated under reduced pressure. The residue is purified by flash column chromatography on silica gel using the indicated mixture of solvents as eluent.

(b) Synthesis of Arylboronic Acids ${ }^{1}$

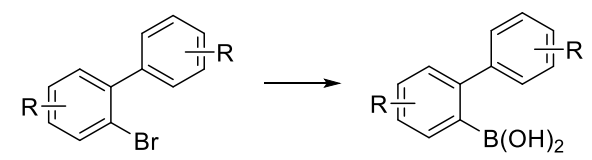

In a $50 \mathrm{~mL}$ Schlenk reaction flask, 2-bromo-1,1'-biphenyl $(10 \mathrm{mmol})$ soluted into $20 \mathrm{~mL}$ THF, and n-BuLi $(1.6 \mathrm{~mol} / \mathrm{L}, 1.5 \mathrm{eq})$ was added slowly at $-78^{\circ} \mathrm{C}$. The mixture was stirred for 2 hours at $-78^{\circ} \mathrm{C}$. Then $\mathrm{B}(\mathrm{OMe})_{3}(3.0 \mathrm{eq})$ was added slowly at $-78^{\circ} \mathrm{C}$. The mixture was stirred for $10 \mathrm{~h}$ from $-78^{\circ} \mathrm{C}$ to 
room temperature. $1 \mathrm{M} \mathrm{HCl}$ was added slowly to control the $\mathrm{pH}$ of the mixture at 6-7. The mixture was then diluted with $20 \mathrm{~mL}$ EtOAc, washed with $40 \mathrm{~mL}$ water, and the aqueous layer was extracted with EtOAc $(3 \times 20 \mathrm{ml})$. All organic extracts were pooled, dried over $\mathrm{MgSO}_{4}$, and concentrated under reduced pressure to leave a residue which was purified by silica gel chromatography with petroleum and ethyl acetate as eluent (PE: EA = 3: 1) to afford arylboronic acids.

Characterizations of substrates:<smiles>FC(F)(F)c1cccc(-c2cccc(C(F)(F)F)c2)c1</smiles>

White solid, $12 \%$ yield for two steps without optimization of the reaction conditions $(0.40 \mathrm{~g})$;

${ }^{1} \mathrm{H}$ NMR (400 MHz, DMSO) $\delta 8.30$ (s, 2H), $7.86-7.65$ (m, 7H). ${ }^{13} \mathrm{C}$ NMR (100 MHz, DMSO) $\delta 143.6$, 142.7, 133.3, 132.5, 129.5, 129.3 (q, $J=31.6 \mathrm{~Hz}, 1 \mathrm{C}), 125.0$ (q, $J=3.9 \mathrm{~Hz}, 1 \mathrm{C}), 124.6$ (q, $J=3.6 \mathrm{~Hz}$, 1C), 124.28 (q, $J=272.4 \mathrm{~Hz}, 1 \mathrm{C}), 124.27$ (q, $J=272.4 \mathrm{~Hz}, 1 \mathrm{C}), 124.2$ (q, $J=3.6 \mathrm{~Hz}, 1 \mathrm{C}), 123.3$ (q, $J$ $=3.7 \mathrm{~Hz}, 1 \mathrm{C}) .{ }^{19} \mathrm{~F}$ NMR $(565 \mathrm{MHz}, \mathrm{DMSO}) \delta-61.16,-61.25$.<smiles>COc1ccc(C)cc1-c1cccc(C)c1</smiles>

White solid, 25\% yield for two steps without optimization of the reaction conditions $(0.56 \mathrm{~g})$; ${ }^{1} \mathrm{H}$ NMR (400 MHz, DMSO) $\delta 7.77$ (s, 2H), 7.33 (d, $\left.J=7.4 \mathrm{~Hz}, 1 \mathrm{H}\right), 7.29-7.18(\mathrm{~m}, 3 \mathrm{H}), 7.15(\mathrm{~s}, 1 \mathrm{H})$, $7.14-7.08$ (m, 2H), 2.34 (s, 3H), 2.33 (s, 3H). $\left.{ }^{13} \mathrm{C} \mathrm{NMR} \mathrm{(100} \mathrm{MHz,} \mathrm{CDCl}_{3}\right) \delta 144.5,143.3,137.3$, 137.0, 132.4, 128.9, 128.9, 128.0, 127.3, 126.6, 125.4, 21.2, 21.0.<smiles>COc1cccc(-c2c(OC)cccc2OC)c1</smiles>

White solid, $4 \%$ yield for two steps without optimization of the reaction conditions $(0.11 \mathrm{~g})$; ${ }^{1} \mathrm{H}$ NMR (600 MHz, DMSO) $\delta 7.38$ (s, 2H), $7.26-7.22(\mathrm{~m}, 2 \mathrm{H}), 7.09$ (dd, $\left.J=7.4,1.7 \mathrm{~Hz}, 1 \mathrm{H}\right), 7.04(\mathrm{~d}$, $J=7.0 \mathrm{~Hz}, 1 \mathrm{H}), 7.00(\mathrm{~d}, J=8.1 \mathrm{~Hz}, 1 \mathrm{H}), 6.96(\mathrm{~d}, J=8.2 \mathrm{~Hz}, 1 \mathrm{H}), 6.89(\mathrm{t}, J=7.4 \mathrm{~Hz}, 1 \mathrm{H}), 3.64(\mathrm{~s}, 3 \mathrm{H})$, 3.62 (s, 3H). ${ }^{13} \mathrm{C}$ NMR $(150 \mathrm{MHz}$, DMSO) $\delta 156.9,156.2,131.4,130.0,128.2,128.0,127.4,124.2$, $119.6,111.4,110.9,55.4,55.1$.<smiles>COc1ccc(Cl)cc1-c1cccc(Cl)c1</smiles>

White solid, 22\% yield for two steps without optimization of the reaction conditions $(0.60 \mathrm{~g})$; ${ }^{1} \mathrm{H}$ NMR (400 MHz, DMSO) $\delta 8.13$ (s, 2H), $7.53-7.36$ (m, 7H). ${ }^{13} \mathrm{C}$ NMR (151 MHz, DMSO) $\delta 144.9$, $143.8,134.2,133.2,132.9,130.1,128.0,127.8,127.2,127.1,126.5$. 
<smiles>COc1ccc(F)cc1-c1cccc(F)c1</smiles>

White solid, $31 \%$ yield for two steps without optimization of the reaction conditions $(0.72 \mathrm{~g})$;

${ }^{1} \mathrm{H}$ NMR (400 MHz, DMSO) $\delta 8.06$ (s, 2H), $7.53-7.44(\mathrm{~m}, 2 \mathrm{H}), 7.31-7.14(\mathrm{~m}, 5 \mathrm{H}) .{ }^{13} \mathrm{C}$ NMR (100 $\left.\mathrm{MHz}, \mathrm{CDCl}_{3}\right) \delta 162.3(\mathrm{~d}, J=244.4 \mathrm{~Hz}, 1 \mathrm{C}), 162.0(\mathrm{~d}, J=243.1 \mathrm{~Hz}, 1 \mathrm{C}), 145.5(\mathrm{dd}, J=7.3,1.9 \mathrm{~Hz}$, 1C), 144.4 (dd, $J=7.7,2.1 \mathrm{~Hz}, 1 \mathrm{C}), 134.6(\mathrm{~d}, J=7.9 \mathrm{~Hz}, 1 \mathrm{C}), 130.2$ (d, $J=8.5 \mathrm{~Hz}, 1 \mathrm{C}), 124.5,124.5$, $115.1(\mathrm{~d}, J=21.8 \mathrm{~Hz}, 1 \mathrm{C}), 115.0(\mathrm{~d}, J=20.6 \mathrm{~Hz}, 1 \mathrm{C}), 114.1(\mathrm{~d}, J=21.0 \mathrm{~Hz}, 1 \mathrm{C}), 113.4$ (d, $J=19.6 \mathrm{~Hz}$, 1C). ${ }^{19} \mathrm{~F}$ NMR $\left(376 \mathrm{MHz}, \mathrm{CDCl}_{3}\right) \delta-108.53--108.60(\mathrm{~m}),-108.79--108.85(\mathrm{~m})$.<smiles>COc1cccc(OC)c1-c1ccccc1F</smiles>

White solid, 5\% yield for two steps without optimization of the reaction conditions $(0.12 \mathrm{~g})$; ${ }^{1} \mathrm{H}$ NMR (600 MHz, DMSO) $\delta 7.98$ (s, 2H), $7.44-7.7 .39$ (m, 2H), 7.377 .37 - 7.36 (m, 1H), $7.34-$ $7.31(\mathrm{~m}, 1 \mathrm{H}), 7.26-7.22(\mathrm{~m}, 3 \mathrm{H}) .{ }^{13} \mathrm{C}$ NMR (151 MHz, DMSO) $\delta 159.5(\mathrm{~d}, J=245.2 \mathrm{~Hz}, 1 \mathrm{C}), 159.0$ (d, $J=245.4 \mathrm{~Hz}, 1 \mathrm{C}), 131.9$ (d, $J=3.1 \mathrm{~Hz}, 1 \mathrm{C}), 129.7$ (d, $J=8.4 \mathrm{~Hz}, 1 \mathrm{C}), 129.3$ (d, $J=7.7 \mathrm{~Hz}, 1 \mathrm{C}$ ), 128.5 (d, $J=3.1 \mathrm{~Hz}, 1 \mathrm{C}), 125.4,125.3,124.0(\mathrm{~d}, J=17.2 \mathrm{~Hz}, 1 \mathrm{C}), 123.9$ (d, $J=3.2 \mathrm{~Hz}, 1 \mathrm{C}), 115.3$ (d, $J=29.9 \mathrm{~Hz}, 1 \mathrm{C}), 115.1(\mathrm{~d}, J=29.5 \mathrm{~Hz}, 1 \mathrm{C}) .{ }^{19} \mathrm{~F}$ NMR (565 MHz, DMSO) $\delta-114.04--114.10(\mathrm{~m})$, $-115.34--115.39(\mathrm{~m})$.

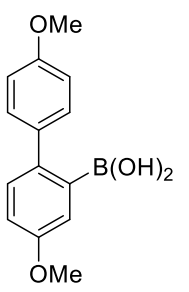

White solid, 16\% yield for two steps without optimization of the reaction conditions $(0.41 \mathrm{~g})$;

${ }^{1} \mathrm{H}$ NMR (600 MHz, MeOD) $\delta 7.32-7.27$ (m, 3H), $6.97-6.92(\mathrm{~m}, 4 \mathrm{H}), 3.797-3.795(\mathrm{~m}, 6 \mathrm{H}) .{ }^{13} \mathrm{C}$ NMR (150 MHz, MeOD) $\delta$ 160.1, 159.4, 138.3, 136.6, 130.5, 130.2, 130.1, 129.7, 121.0, 118.1, 115.4, 114.9, 55.52, 55.47.

(c) Synthesis of trifluoromethyl alkene derivatives ${ }^{2}$

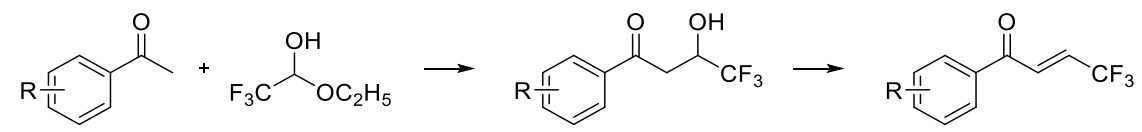

Trifluoroacetaldehyde ethyl hemiacetal was dissolved in toluene, pyrrolidine was added and stirred at room temperature for $30 \mathrm{~min}$. finally, acetophenone compounds were added. The reaction mixture was stirred at $100^{\circ} \mathrm{C}$ for $24 \mathrm{~h}$. After cooling to room temperature, water is added, and the product was extracted into EtOAc. The combined organic extracts were washed with brine, dried over $\mathrm{MgSO} 4$, and concentrated under vacuum. The residue was then purified by flash chromatography on silica gel to 
provide the intermediate, Dissolve intermediate the product in DCM at room temperature, add $4.1 \mathrm{~mL}$ trifluoroacetic anhydride, and finally add $6 \mathrm{~mL}$ triethylamine dropwise, the reaction mixture is stirred at room temperature for $12 \mathrm{~h}$, The residue was then purified by flash chromatography on silica gel to provide the corresponding product.

Characterizations of substrates:<smiles>CS(=O)(=O)c1ccc(C(=O)C=CC(F)(F)F)cc1</smiles>

White solid, $67 \%$ yield $(1.87 \mathrm{~g})$;

${ }^{1} \mathrm{H}$ NMR $\left(400 \mathrm{MHz}, \mathrm{CDCl}_{3}\right) \delta 8.21-8.03(\mathrm{~m}, 4 \mathrm{H}), 7.53-7.48(\mathrm{~m}, 1 \mathrm{H}), 6.92-6.83(\mathrm{~m}, 1 \mathrm{H}), 3.10(\mathrm{~s}$, $3 \mathrm{H}) .{ }^{13} \mathrm{C}$ NMR $\left(100 \mathrm{MHz}, \mathrm{CDCl}_{3}\right) \delta 187.2,145.2,140.2,132.0(\mathrm{q}, J=35.6 \mathrm{~Hz}, 1 \mathrm{C}), 130.4(\mathrm{q}, J=5.5$ $\mathrm{Hz}, 1 \mathrm{C}), 129.7,128.3,122.4$ (q, $J=270.6 \mathrm{~Hz}, 1 \mathrm{C}), 44.4 .{ }^{19} \mathrm{~F}$ NMR $\left(565 \mathrm{MHz}, \mathrm{CDCl}_{3}\right) \delta-65.24$ (d).<smiles>O=C(/C=C/C(F)(F)F)c1ccccc1C(F)(F)F</smiles>

yellow oily liquid, $19 \%$ yield $(0.50 \mathrm{~g})$;

${ }^{1} \mathrm{H}$ NMR (600 MHz, $\left.\mathrm{CDCl}_{3}\right) \delta 7.79-7.75(\mathrm{~m}, 1 \mathrm{H}), 7.69-7.63(\mathrm{~m}, 2 \mathrm{H}), 7.47-7.46(\mathrm{~m}, 1 \mathrm{H}), 7.05-$ $7.02(\mathrm{~m}, 1 \mathrm{H}), 6.49-6.43(\mathrm{~m}, 1 \mathrm{H}) .{ }^{13} \mathrm{C} \mathrm{NMR}\left(150 \mathrm{MHz}, \mathrm{CDCl}_{3}\right) \delta 192.2,137.0(\mathrm{q}, J=1.8 \mathrm{~Hz}, 1 \mathrm{C})$, $134.71(\mathrm{q}, J=5.5 \mathrm{~Hz}, 1 \mathrm{C}), 134.71$ (q, $J=5.6 \mathrm{~Hz}, 1 \mathrm{C}) .132 .2$ (q, $J=35.6 \mathrm{~Hz}, 1 \mathrm{C}), 132.2,131.3,128.28$, 128.25 (q, $J=32.6 \mathrm{~Hz}, 1 \mathrm{C}), 127.2$ (q, $J=4.8 \mathrm{~Hz}, 1 \mathrm{C}), 123.5$ (q, $J=273.8 \mathrm{~Hz}, 1 \mathrm{C}), 122.3(\mathrm{q}, J=270.7$ $\mathrm{Hz}, 1 \mathrm{C}) .{ }^{19} \mathrm{~F}$ NMR $\left(565 \mathrm{MHz}, \mathrm{CDCl}_{3}\right) \delta-57.89,-65.52(\mathrm{~d})$.

\section{Experimental Section}

\section{(1) Optimizations of the model reaction}

Table S1 Optimizations of the model reaction ${ }^{\mathrm{a}}$
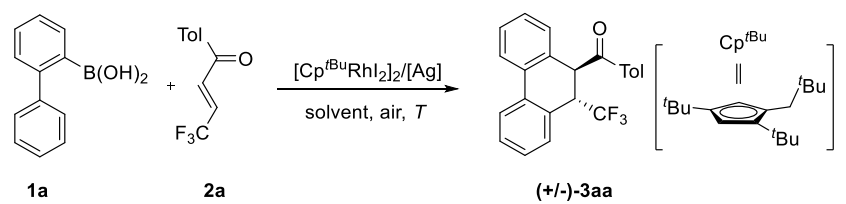

$\begin{array}{cccc}\text { entry } & \text { oxidant (x equiv) } & \text { solvent }(1 \mathrm{~mL}) & \text { yield } \% \\ 1 & \operatorname{AgOAc}(2.0) & \text { EtOAc } & 39 \\ 2 & \operatorname{AgOAc}(2.0) & \mathrm{TFE} . \\ 3 & \operatorname{AgOAc}(2.0) & \mathrm{DCE} & 38 \\ 4 & \operatorname{AgOAc}(2.0) & \mathrm{THF} & 26 \\ 5 & \mathrm{Ag}_{2} \mathrm{CO}_{3}(2.0) & \text { EtOAc } & 54 \\ 6 & \mathrm{Ag}_{2} \mathrm{O}(2.0) & \text { EtOAc } & 68\end{array}$




$\begin{array}{cccc}7 & \operatorname{AgOAc}(0.25), \mathrm{Ag}_{2} \mathrm{O}(1.0) & \text { EtOAc } & 77 \\ 8^{\mathrm{b}} & \operatorname{AgOAc}(0.25), \mathrm{Ag}_{2} \mathrm{O}(1.0) & \text { EtOAc } & 38(28)^{\mathrm{c}} \\ 9^{\mathrm{d}} & \operatorname{AgOAc}(0.25), \mathrm{Ag}_{2} \mathrm{O}(1.0) & \text { EtOAc } & 90 \\ 10^{\mathrm{d}, \mathrm{e}} & \operatorname{AgOAc}(0.25), \mathrm{Ag}_{2} \mathrm{O}(1.0) & \text { EtOAc } & 94 \\ 11^{\mathrm{f}} & \operatorname{AgOAc}(0.25), \mathrm{Ag}_{2} \mathrm{O}(1.0) & \text { EtOAc } & \text { n.r. }\end{array}$

${ }^{\mathrm{a}}$ Reaction conditions: 1a $(0.1 \mathrm{mmol}), \mathbf{2 a}(0.1 \mathrm{mmol}),\left[\mathrm{Cp}^{t \mathrm{Bu}} \mathrm{RhI}_{2}\right]_{2}(4 \mathrm{~mol} \%)$, oxidant (x equiv), solvent (1 $\mathrm{mL}), 60{ }^{\circ} \mathrm{C}, 12 \mathrm{~h}$, under air, isolated yield. ${ }^{\mathrm{b}}\left[\mathrm{Cp}^{*} \mathrm{RhCl}_{2}\right]_{2}(4 \mathrm{~mol} \%)$ was used instead of $\left[\mathrm{Cp}^{\mathrm{t} B \mathrm{Ru}} \mathrm{RhI}_{2}\right]_{2} \cdot{ }^{\mathrm{c}} \mathrm{Yield}$ of the non-annulative 1,4 -addition product. ${ }^{\mathrm{d}} 80{ }^{\circ} \mathrm{C} .{ }^{\mathrm{e}} \mathbf{1} \mathbf{a}(0.12 \mathrm{mmol}) .{ }^{\mathrm{f}}$ Without $\left[\mathrm{Cp}^{\mathrm{tBu}} \mathrm{RhI}_{2}\right]_{2}$, n.r. $=$ no reaction.

\section{(2) General procedures for the synthesis of products 3.}

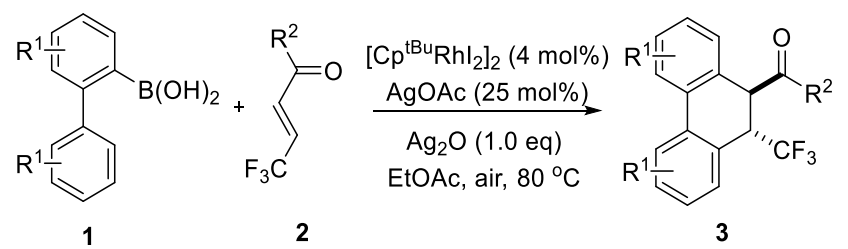

A mixture of $1(0.12 \mathrm{mmol}), 2(0.1 \mathrm{mmol}),\left[\mathrm{Cp}^{t{ }^{\mathrm{Bu}}} \mathrm{RhI}_{2}\right]_{2}$ (4.9 mg, $\left.0.002 \mathrm{mmol}, 2 \mathrm{~mol} \%\right), \operatorname{AgOAc}(4.2$ $\mathrm{mg}, 0.25 \mathrm{mmol}, 25 \mathrm{~mol} \%), \mathrm{Ag}_{2} \mathrm{O}(23.2 \mathrm{mg}, 0.1 \mathrm{mmol}, 1.0 \mathrm{eq})$, and EtOAc (1.0 mL) were charged into a reaction tube. The reaction mixture was stirred at $80{ }^{\circ} \mathrm{C}$ heated by metal sand bath for $12 \mathrm{~h}$. After the solvent was removed under reduced pressure, the residue was purified by silica gel chromatography to afford 3 .

(3) General procedures for the synthesis of products 5.
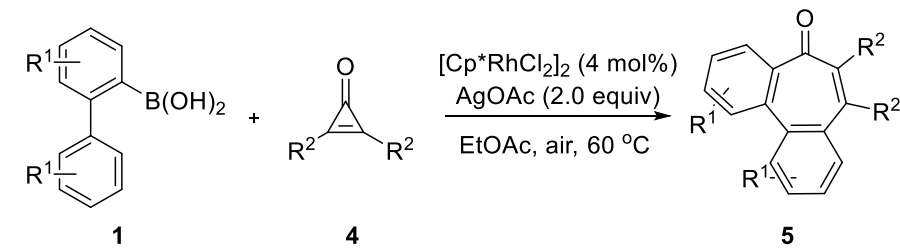

A mixture of 1 (0.15 mmol), 4 (0.1 mmol), [Cp $\left.{ }^{*} \mathrm{RhCl}_{2}\right]_{2}(2.5 \mathrm{mg}, 0.004 \mathrm{mmol}, 4 \mathrm{~mol} \%), \mathrm{AgOAc}(33.4$ $\mathrm{mg}, 0.2 \mathrm{mmol}, 2.0 \mathrm{eq})$ and EtOAc $(1.0 \mathrm{~mL})$ were charged into a reaction tube. The reaction mixture was stirred at $60{ }^{\circ} \mathrm{C}$ heated by metal sand bath for $12 \mathrm{~h}$. After the solvent was removed under reduced pressure, the residue was purified by silica gel chromatography to afford $\mathbf{5}$.

(4) Scale-up Synthesis of the Product 3aa.

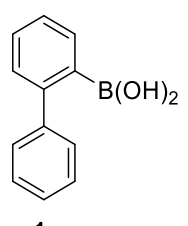

$1 \mathrm{a}$

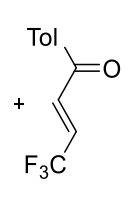

$2 \mathrm{a}$

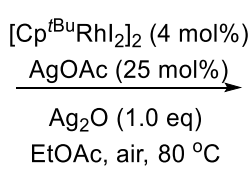

EtOAc, air, $80^{\circ} \mathrm{C}$

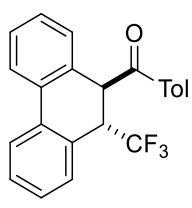

3aa

A mixture of $\mathbf{1 a}(800.0 \mathrm{mg}, 4.0 \mathrm{mmol}), \mathbf{2 a}(428.0 \mathrm{mg}, 2.0 \mathrm{mmol}),\left[\mathrm{Cp}^{t \mathrm{Bu}^{2}} \mathrm{RhI}_{2}\right]_{2}(49.0 \mathrm{mg}, 0.04 \mathrm{mmol}, 2$ mol\%), AgOAc (84.0 mg, $0.5 \mathrm{mmol}, 25 \mathrm{~mol} \%), \mathrm{Ag}_{2} \mathrm{O}$ (464.0 mg, $\left.2.0 \mathrm{mmol}, 1.0 \mathrm{eq}\right)$, and EtOAc (40.0 $\mathrm{mL}$ ) were charged into a reaction tube. The reaction mixture was stirred at $80{ }^{\circ} \mathrm{C}$ heated by metal sand bath for $12 \mathrm{~h}$. After the solvent was removed under reduced pressure, the residue was purified by silica 
gel chromatography using PE/ EtOAc $=10: 1$ to afford 3aa $(600 \mathrm{mg}, 82 \%$ yiled; $106 \mathrm{mg}$, 29\% yiled,

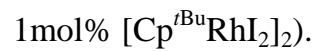

(5) Scale-up Synthesis of the Product 5aa.

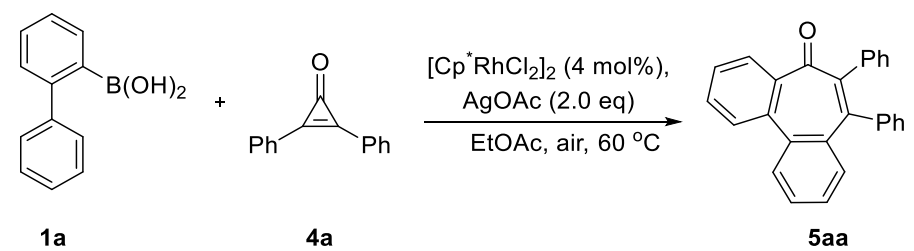

A mixture of $\mathbf{1 a}(300.0 \mathrm{mg}, 1.5 \mathrm{mmol}), \mathbf{4 a}(206.0 \mathrm{mg}, 1.0 \mathrm{mmol}),\left[\mathrm{Cp}^{*} \mathrm{RhCl}_{2}\right]_{2}(25.0 \mathrm{mg}, 0.4 \mathrm{mmol}, 4$ mol\%), AgOAc (334.0 mg, $2.0 \mathrm{mmol}, 2.0 \mathrm{eq})$ and EtOAc $(10.0 \mathrm{~mL})$ were charged into a reaction tube. The reaction mixture was stirred at $60{ }^{\circ} \mathrm{C}$ heated by metal sand bath for $4.5 \mathrm{~h}$. After the solvent was removed under reduced pressure, the residue was purified by silica gel chromatography using PE/ EtOAc $=30: 1$ to afford 5aa $\left(304.3 \mathrm{mg}, 85 \%\right.$ yield; $298 \mathrm{mg}, 83 \%, 2 \mathrm{~mol} \%\left[\mathrm{Cp}^{*} \mathrm{RhCl}_{2}\right]_{2} ; 184 \mathrm{mg}, 51 \%, 1$ $\left.\mathrm{mol} \%\left[\mathrm{Cp}^{*} \mathrm{RhCl}_{2}\right]_{2}\right)$.

(6) Synthetic Transformation of the Product 3aa.

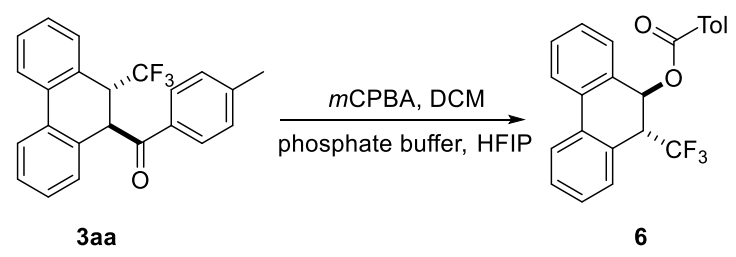

3aa $(36.6 \mathrm{mg}, 0.1 \mathrm{mmol}), m \mathrm{CPBA}(83.0 \mathrm{mg}, 0.5 \mathrm{mmol})$ were dissolved in $\mathrm{CH}_{2} \mathrm{Cl}_{2}(1.0 \mathrm{~mL})$, Then phosphate buffer $(\mathrm{PH}=7,0.4 \mathrm{~mL})$ and HFIP $(1.0 \mathrm{~mL})$ were added and the room temperature was violently stirred for $36 \mathrm{~h}$. The organic phase was quenched with sat. aq. $\mathrm{Na}_{2} \mathrm{~S}_{2} \mathrm{O}_{3}(3.0 \mathrm{~mL})$, extracted with $\mathrm{CH}_{2} \mathrm{Cl}_{2}$, dried over $\mathrm{Na}_{2} \mathrm{SO}_{4}$, filtered, and the solvent removed in vacuo. Purification by PE/ EtOAc $=30: 1$ afforded $\mathbf{6}(14.1 \mathrm{mg}, 37 \%$ yield $)$.

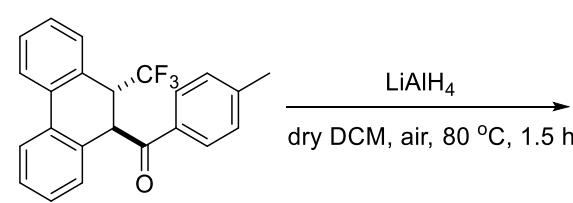

$3 a a$

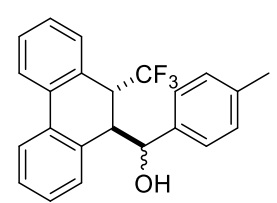

$7+7$

3aa (36.6mg, $0.1 \mathrm{mmol})$ was dissolved in dry DCM $(2.0 \mathrm{~mL})$ and $\mathrm{LiAlH}_{4}(29.0 \mathrm{mg}, 1.0 \mathrm{mmol})$ was added and the reaction mixture was stirred at $80{ }^{\circ} \mathrm{C}$ for another $4 \mathrm{~h}$ and then quenched with water $(2.0$ $\mathrm{mL}$ ) by slow addition. The reaction mixture was firstly filtered, Evaporation of the solvent followed by purification by flash chromatography on silica gel (PE) gave the corresponding product 7+7' (7, 17.7 mg, $48 \%$ yiled as off-white solid; 7', $16.6 \mathrm{mg}, 45 \%$ yiled as off-white solid).

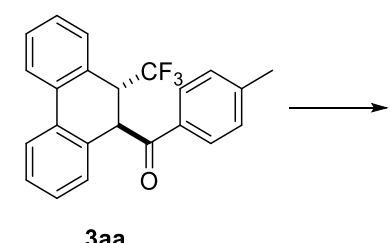

$3 a a$

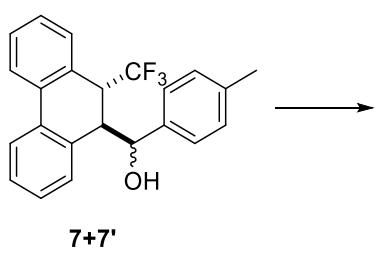

$\mathbf{7 + 7}^{\prime}$<smiles>FC(F)(F)c1c(CCl)c2ccccc2c2ccccc12</smiles>

8 
7+7' (35.6 mg, $0.1 \mathrm{mmol}$ ) were dissolved in dry DCM, then trifluorosulfonic acid (15.0 mg, $0.1 \mathrm{mmol})$ was add along the tube wall. The reaction was heated at $80{ }^{\circ} \mathrm{C}$ and stirred for $12 \mathrm{~h}$ and then cooled to room temperature. Solvents were removed in vacuo and Evaporation of the solvent followed by purification by flash chromatography on silica gel (hexane) gave the corresponding product $\mathbf{8}(29.3 \mathrm{mg}$, $84 \%$ yiled).

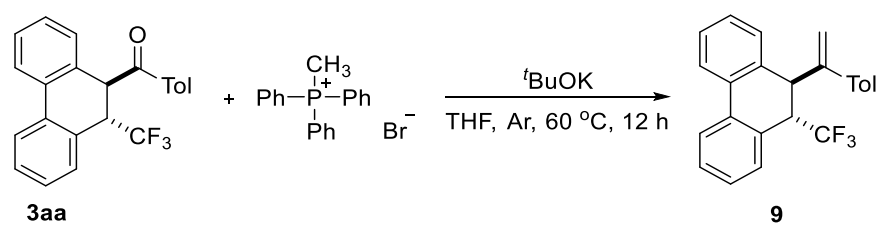

To a solution of methyltriphenyl phosphonium bromide $(124.0 \mathrm{mg}, 0.4 \mathrm{mmol})$ and potassium tert-butoxide $(44.9 \mathrm{mg}, 0.4 \mathrm{mmol})$ in THF $(2.0 \mathrm{~mL})$ under Ar atmosphere was added the acyl derivative (36.6 mg, $0.1 \mathrm{mmol}$ ). The reaction was heated at $30{ }^{\circ} \mathrm{C}$ and stirred for $12 \mathrm{~h}$ and then cooled to room temperature. Solvents were removed in vacuo and the resulting mixture was extracted with diethyl ether. The combined organic layers were washed with brine and dried over anhydrous sodium sulfate. Evaporation of the solvent followed by purification by flash chromatography on silica gel (PE/ EtOAc $=30: 1)$ gave the corresponding product 9 (29.2 $\mathrm{mg}, 80 \%$ yiled, white solide $)$.

\section{(7) Synthetic Transformation of the Product 5aa.}

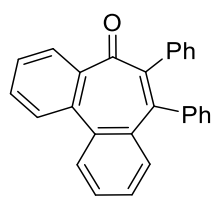

5 aa

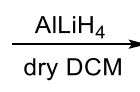

5aa $(35.8 \mathrm{mg}, 0.1 \mathrm{mmol})$ was dissolved in dry DCM $(1.0 \mathrm{~mL})$ and $\mathrm{LiAlH}_{4}(29.0 \mathrm{mg}, 0.1 \mathrm{mmol})$ was added and the reaction mixture was stirred at $80{ }^{\circ} \mathrm{C}$ for another $4 \mathrm{~h}$ and then quenched with water $(2.0$ $\mathrm{mL}$ ) by slow addition. The reaction mixture was firstly filtered, Evaporation of the solvent followed by purification by flash chromatography on silica gel chromatography (PE) gave the corresponding product $10(35.5 \mathrm{mg}, 99 \%)$.

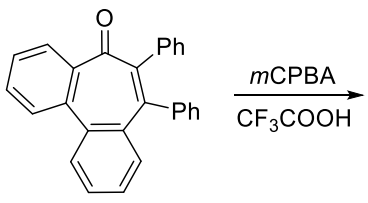

5aa

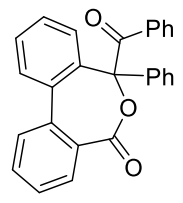

11

5aa $(35.8 \mathrm{mg}, 0.1 \mathrm{mmol}), m \mathrm{CPBA}(83.0 \mathrm{mg}, 0.5 \mathrm{mmol}), \mathrm{CF}_{3} \mathrm{COOH}(16 \mu \mathrm{L}, 0.2 \mathrm{mmol}$ were dissolved in $\mathrm{CH}_{2} \mathrm{Cl}_{2}(1.0 \mathrm{~mL})$ and the reaction mixture was stirred for another $15 \mathrm{~h}$. Evaporation of the solvent followed by purification by silica gel chromatography using $\mathrm{PE} / \mathrm{EtOAc}=30: 1$ to gave the corresponding product 11 (20.1 mg, 54\% yiled).

(8) General procedures for the synthesis of intermediate 3-I. 


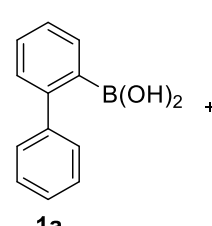

$1 \mathrm{a}$

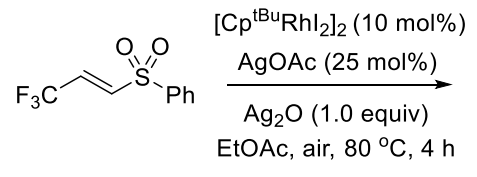
EtOAc, air, $80^{\circ} \mathrm{C}, 4 \mathrm{~h}$

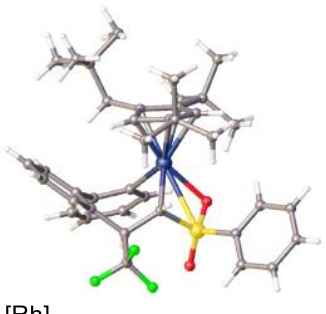

3-I, $40 \%$ based on [Rh]

A mixture of 1a (23.8 mg, $0.12 \mathrm{mmol})$, ((3,3,3-trifluoroprop-1-en-1-yl)sulfonyl)benzene (23.6 mg, 0.1 mmol), $\left[\mathrm{Cp}^{t \mathrm{Bu}} \mathrm{RhI}_{2}\right]_{2}(12.25 \mathrm{mg}, 0.01 \mathrm{mmol}, 10 \mathrm{~mol} \%)$, AgOAc (4.2 mg, $\left.0.25 \mathrm{mmol}, 25 \mathrm{~mol} \%\right), \mathrm{Ag}_{2} \mathrm{O}$ $(23.2 \mathrm{mg}, 0.1 \mathrm{mmol}, 1.0 \mathrm{eq})$, and EtOAc $(1.0 \mathrm{~mL})$ were charged into a reaction tube. The reaction mixture was stirred at $80{ }^{\circ} \mathrm{C}$ heated by metal sand bath for $4 \mathrm{~h}$. After the solvent was removed under reduced pressure, the residue was purified by silica gel chromatography to afford 3-I (6.1 $\mathrm{mg}, 40 \%$ yield).

\section{(9) Mechanistic Studies}

(a) H/D Exchange experiment of 1a with 2a<smiles>Oc1ccccc1-c1ccccc1</smiles>

$1 a$<smiles>O=C(Cl)/C=C/C(F)(F)F</smiles>

2a

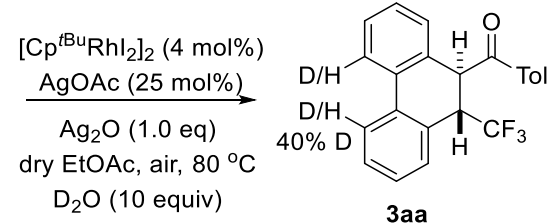

3 aa

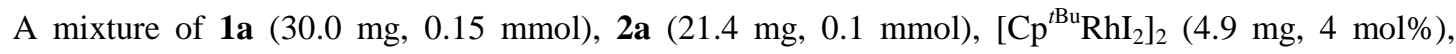
AgOAc (4.2 mg, $0.025 \mathrm{mmol}, 0.25 \mathrm{eq}), \mathrm{Ag}_{2} \mathrm{O}$ (23.2 mg, $\left.0.1 \mathrm{mmol}, 1.0 \mathrm{eq}\right), \mathrm{D}_{2} \mathrm{O}$ (18.0 mg, 1.0 mmol,10.0 eq) and dry EtOAc $(1.0 \mathrm{~mL})$ were charged into a reaction tube. The reaction mixture was stirred under air at $80{ }^{\circ} \mathrm{C}$ heated by metal sand bath for $12 \mathrm{~h}$. After the solvent was removed under reduced pressure, the residue was purified by silica gel chromatography using PE/ EtOAc $=(20: 1$ to 10:1) to afford 3aa in 39\% yield . ${ }^{1} \mathrm{H}$ NMR analysis indicated $40 \%$ deuteration at the ortho position of the biphenyl rings.

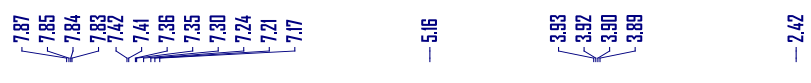

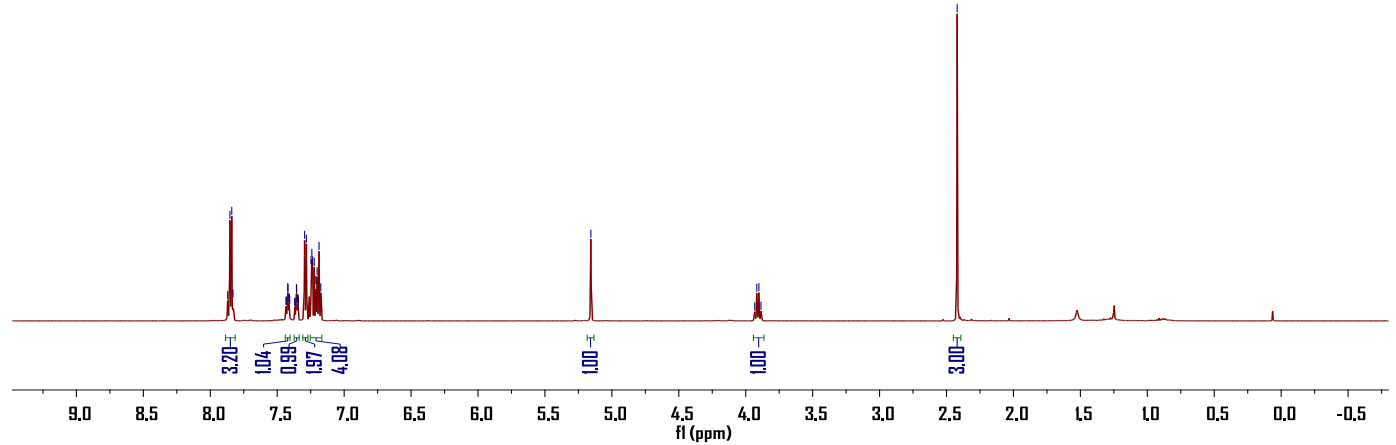

(b) H/D Exchange experiment of 1c with 2a 

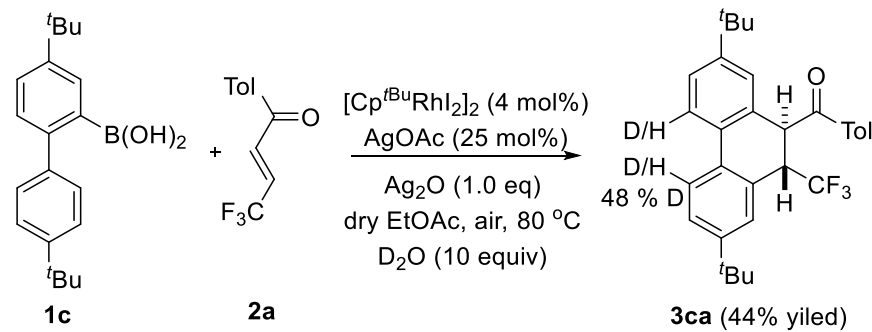

A mixture of $1 \mathbf{c}(46.5 \mathrm{mg}, 0.15 \mathrm{mmol}), \mathbf{2 a}(21.4 \mathrm{mg}, 0.1 \mathrm{mmol}),\left[\mathrm{Cp}^{t \mathrm{Bu}} \mathrm{RhI}_{2}\right]_{2}(4.9 \mathrm{mg}, 4 \mathrm{~mol} \%)$, $\mathrm{AgOAc}(4.2 \mathrm{mg}, 0.025 \mathrm{mmol}, 0.25 \mathrm{eq}), \mathrm{Ag}_{2} \mathrm{O}(23.2 \mathrm{mg}, 0.1 \mathrm{mmol}, 1.0 \mathrm{eq}), \mathrm{D}_{2} \mathrm{O}$ (18.0 mg, 1.0 $\mathrm{mmol}, 10.0 \mathrm{eq})$ and dry EtOAc $(1.0 \mathrm{~mL})$ were charged into a reaction tube. The reaction mixture was stirred under air at $80{ }^{\circ} \mathrm{C}$ heated by metal sand bath for $12 \mathrm{~h}$. After the solvent was removed under reduced pressure, the residue was purified by silica gel chromatography using PE/ EtOAc $=(20: 1$ to 10:1) to afford 3ca in $44 \%$ yield ${ }^{1} \mathrm{H}$ NMR analysis indicated $48 \%$ deuteration at the ortho position of the biphenyl rings.
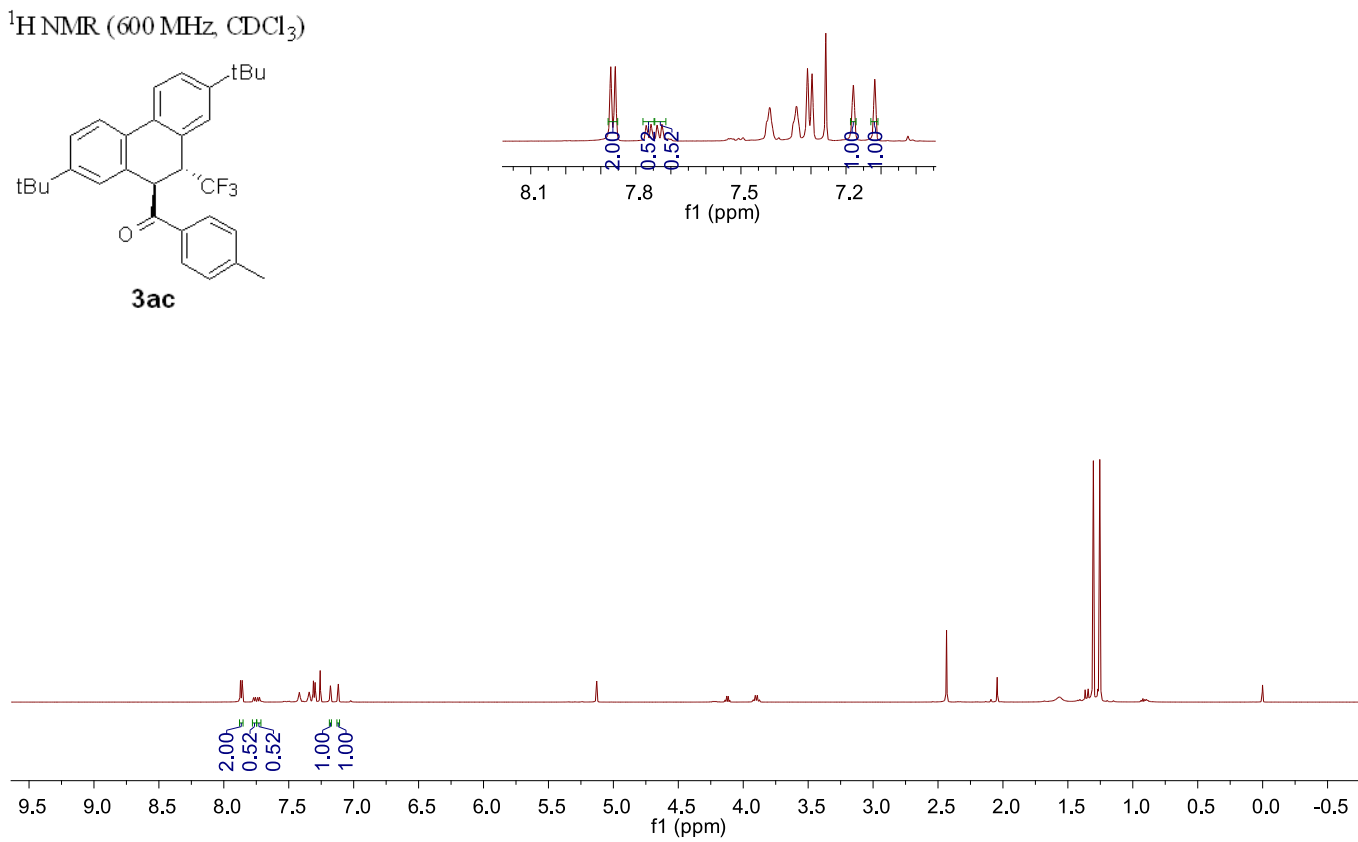

(c) Determination of Kinetic Isotope Effects

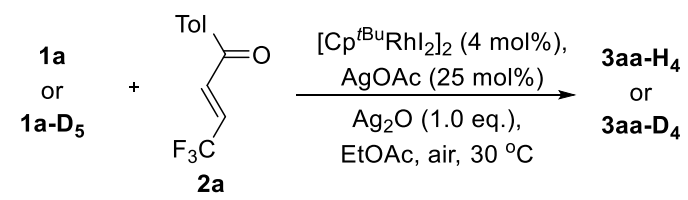

For the cyclization of [1a-H $\mathbf{5}]$ : a $25-\mathrm{mL}$ tube equipped with a magnetic stir bar was charged with $\mathbf{2 a}$ (21.4 mg, $0.1 \mathrm{mmol}, 1.0 \mathrm{eq})\left[\mathrm{Cp}^{t \mathrm{Bu}} \mathrm{RhI}_{2}\right]_{2}(4.9 \mathrm{mg}, 4 \mathrm{~mol} \%), \mathrm{Ag}_{2} \mathrm{O}$ (23.2 mg, $\left.0.1 \mathrm{mmol}, 1.0 \mathrm{eq}\right)$, $\mathrm{AgOAc}(4.2 \mathrm{mg}, 0.025 \mathrm{mmol}, 0.25 \mathrm{eq})$ and $1 \mathrm{~mL}$ EtOAc, and $\mathbf{1 a - \mathbf { H } _ { 5 }}(0.12 \mathrm{mmol}, 1.2$ equiv) were added finally. The tube was capped with a Teflon-lined screw cap. The sealed tube was placed in metal sand bath at $30{ }^{\circ} \mathrm{C}$. After 10 minutes of reaction, the tube was removed from the metal sand bath, the resulting solution was filtered with a PTE filter and the solvent was removed under reduced pressure. the resulting mixture were diluted with $\mathrm{CDCl}_{3}, 10 \mu \mathrm{L}$ of benzotrifluoride was added and The mixed 
solution was transferred to an NMR tube and the sample was analyzed by ${ }^{19} \mathrm{~F}$ NMR. The amount of 3aa- $\mathbf{H}_{4}$ was determined by the relative integration of the characteristic signals of benzotrifluoride and 3aa- $\mathbf{H}_{4}$, The experiment was repeated at 20,30 and 40 minutes. The experiments were performed by duplicate.

For the cyclization of [1a-D $\mathbf{5}]$ : a $25-\mathrm{mL}$ tube equipped with a magnetic stir bar was charged with $\mathbf{2 a}$ (21.4 mg, $0.1 \mathrm{mmol}, 1.0 \mathrm{eq}$ ), [C $\left.{ }^{\mathrm{tBu}} \mathrm{RhI}_{2}\right]_{2}$ (4.9 mg, $4 \mathrm{~mol} \%$ ), $\mathrm{Ag}_{2} \mathrm{O}$ (23.2 mg, $0.1 \mathrm{mmol}, 1.0 \mathrm{eq}$ ), $\mathrm{AgOAc}(4.2 \mathrm{mg}, 0.025 \mathrm{mmol}, 0.25 \mathrm{eq})$ and $1 \mathrm{~mL}$ EtOAc and $\mathbf{1} \mathbf{a}-\mathbf{D}_{\mathbf{5}}$ ( $0.12 \mathrm{mmol}, 1.2$ equiv) were added finally. The tube was capped with a Teflon-lined screw cap. The sealed tube was placed in metal sand bath at $30{ }^{\circ} \mathrm{C}$. After 10 minutes of reaction, the tube was removed from the metal sand bath, the resulting solution was filtered with a PTE filter and the solvent was removed under reduced pressure. the resulting mixture were diluted with $\mathrm{CDCl}_{3}, 10 \mu \mathrm{L}$ of benzotrifluoride was added and The mixed solution was transferred to an NMR tube and the sample was analyzed by ${ }^{19} \mathrm{~F}$ NMR. The amount of 3aa- $\mathbf{D}_{4}$ was determined by the relative integration of the characteristic signals of benzotrifluoride and 3aa- $\mathbf{D}_{4}$, The experiment was repeated at 20,30 and 40 minutes. The experiments were performed by duplicate.

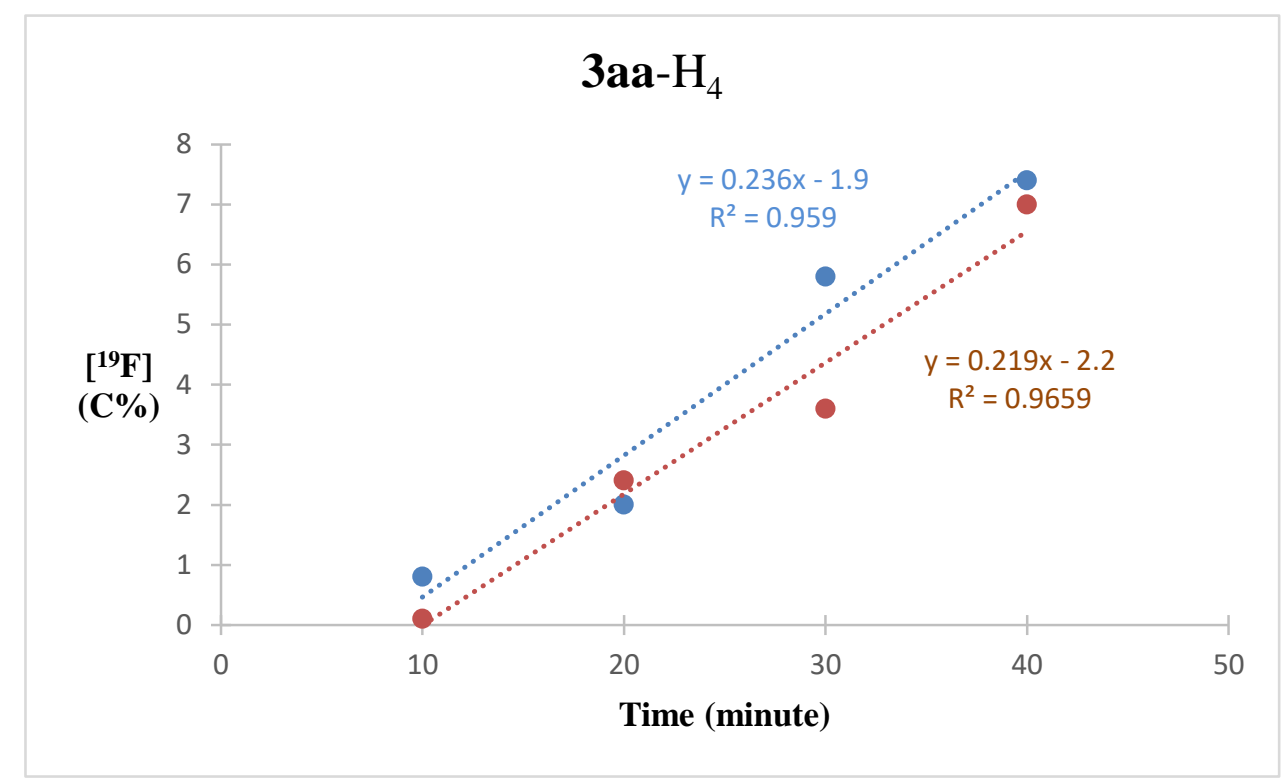

Initial rates (two parallel reactions) determined by plots of [3aa- $\left.\mathbf{H}_{4}\right]$ versus time, which gave an average value of $0.2275 \times 10^{-4} \mathrm{M} / \mathrm{min}$. 


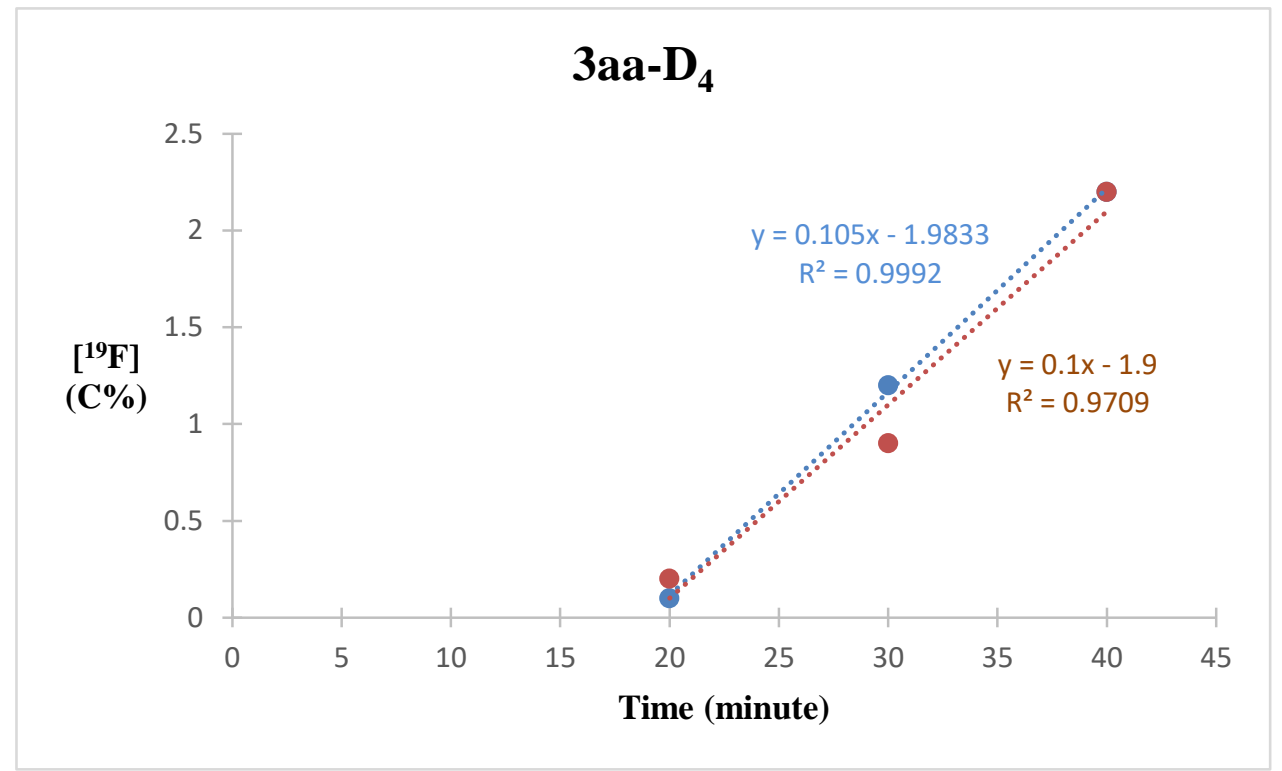

Initial rates (two parallel reactions) determined by plots of [3aa- $\left.\mathbf{D}_{\mathbf{4}}\right]$ versus time, which gave an average value of $0.1 \times 10^{-4} \mathrm{M} / \mathrm{min}$.

The KIE value measured based on the above experiments is $\mathbf{2 . 3}$.

\section{Possible mechnisms for formation of 3 wa and 5}
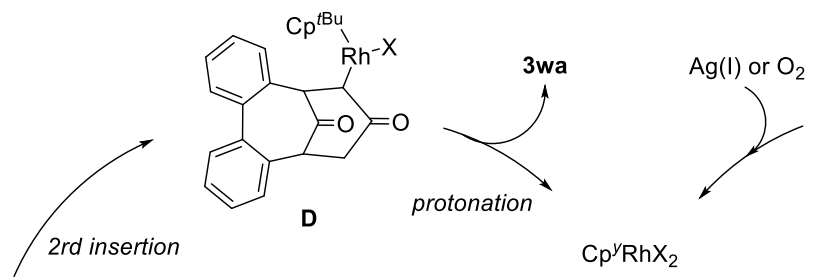

$\mathrm{Cp}{ }^{*} \mathrm{Rh}(\mathrm{I})$
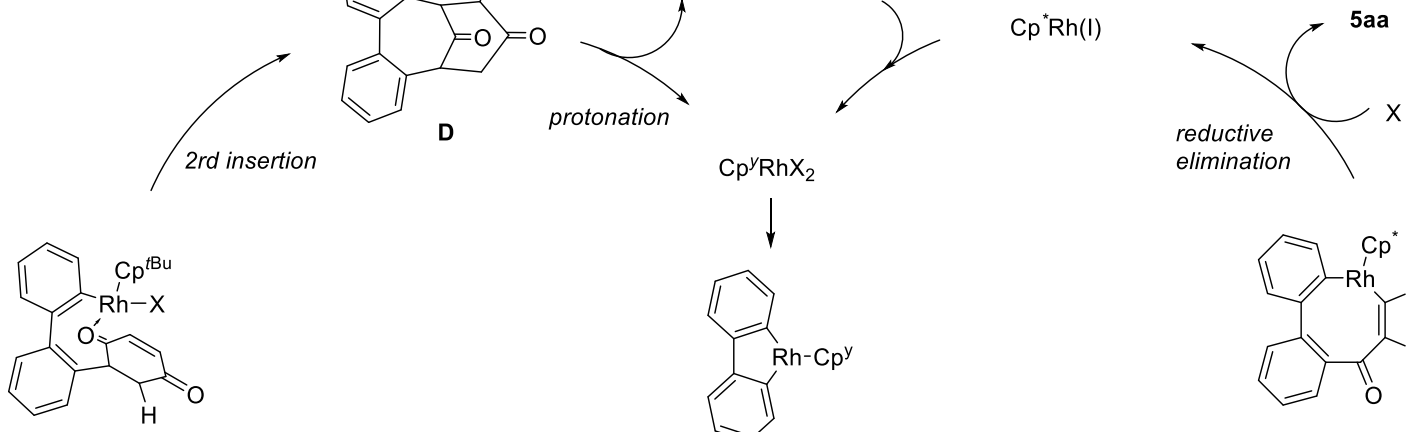

$\mathrm{Cp}^{y} \mathrm{RhX}_{2}$

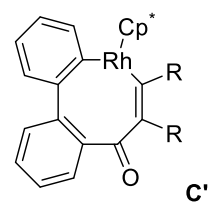

C
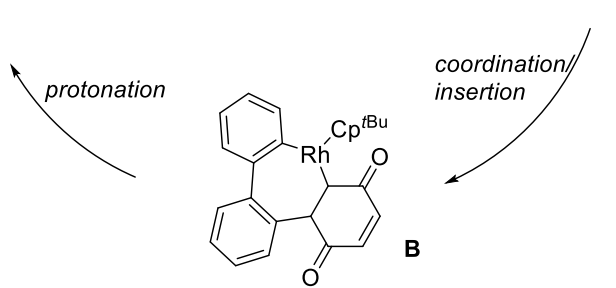

A

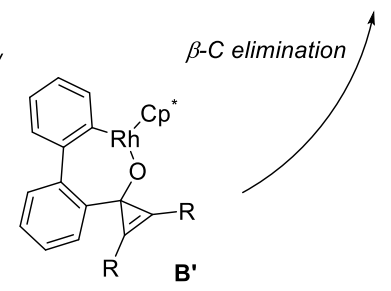




\section{X-Ray Crystal Structures}

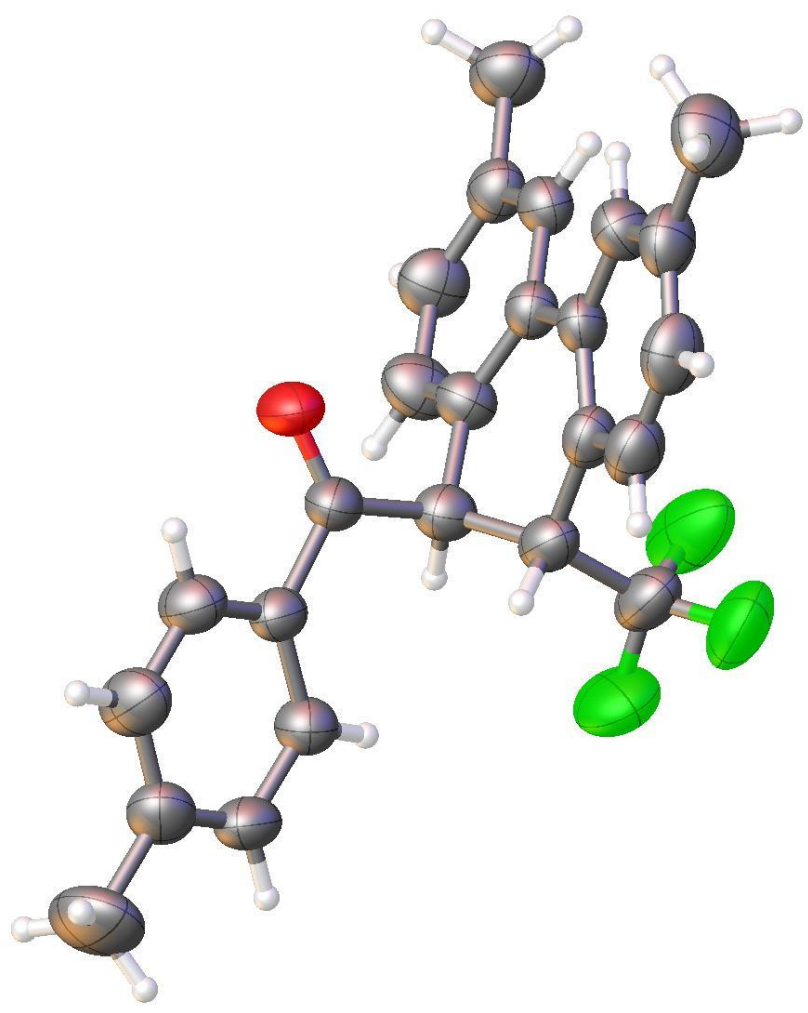

Thermal ellipsoids are set at the 50\% probability level.

Table S2 Crystal data and structure refinement for 3ae.

Identification code

Empirical formula

Formula weight

Temperature/K

Crystal system

Space group

$\mathrm{a} / \AA$

$\mathrm{b} / \AA$

$\mathrm{c} / \AA$

$\alpha /{ }^{\circ}$

$\beta /{ }^{\circ}$

$\gamma /{ }^{\circ}$

Volume/ $\AA^{3}$

Z

$\rho_{\text {calc }} \mathrm{g} / \mathrm{cm}^{3}$

$\mu / \mathrm{mm}^{-1}$

$\mathrm{F}(000)$
YLY-1-20210506

$\mathrm{C}_{25} \mathrm{H}_{21} \mathrm{~F}_{3} \mathrm{O}$

394.42

293(2)

monoclinic

$\mathrm{P} 2{ }_{1} / \mathrm{c}$

12.5924(13)

13.7442(10)

12.8939(15)

90

113.804(13)

90

2041.7(4)

4

1.283

0.096

824.0 
Crystal size $/ \mathrm{mm}^{3}$

Radiation

$2 \Theta$ range for data collection ${ }^{\circ}$

Index ranges

Reflections collected

Independent reflections

Data/restraints/parameters

Goodness-of-fit on $\mathrm{F}^{2}$

Final $\mathrm{R}$ indexes $[\mathrm{I}>=2 \sigma(\mathrm{I})]$

Final $\mathrm{R}$ indexes [all data]

Largest diff. peak/hole / e $\AA^{-3}$
$0.5 \times 0.3 \times 0.2$

Mo $\operatorname{K} \alpha(\lambda=0.71073)$

6.862 to 58.096

$-16 \leq \mathrm{h} \leq 16,-18 \leq \mathrm{k} \leq 18,-17 \leq 1 \leq 16$

13688

$4800\left[\mathrm{R}_{\mathrm{int}}=0.1305, \mathrm{R}_{\text {sigma }}=0.1454\right]$

$4800 / 0 / 265$

0.966

$\mathrm{R}_{1}=0.0838, \mathrm{wR}_{2}=0.2008$

$\mathrm{R}_{1}=0.1649, \mathrm{wR}_{2}=0.2440$

$0.24 /-0.24$

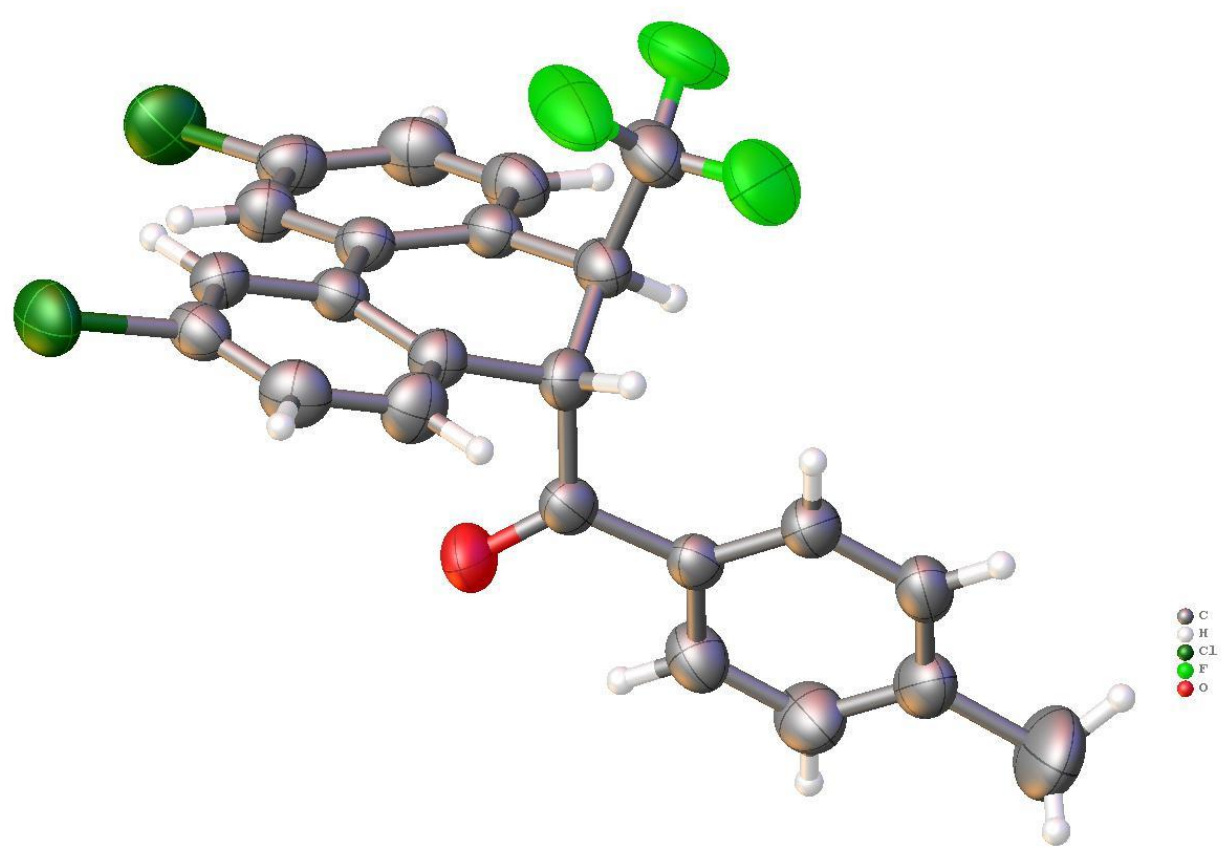

Thermal ellipsoids are set at the $50 \%$ probability level.

Table S3 Crystal data and structure refinement for 3af.

Identification code

Empirical formula

Formula weight

Temperature/K

Crystal system

Space group

$\mathrm{a} / \AA$

b/A

c/

$\alpha /^{\circ}$
YLY-20210507

$\mathrm{C}_{23} \mathrm{H}_{15} \mathrm{Cl}_{2} \mathrm{~F}_{3} \mathrm{O}$

435.25

293.0

monoclinic

$\mathrm{P} 2{ }_{1} / \mathrm{c}$

$12.2926(8)$

13.8685(6)

12.6728(7)

90 
$\beta /{ }^{\circ}$

$\gamma^{\circ}$

Volume $/ \AA^{3}$

$\mathrm{Z}$

$\rho_{\text {calc }} \mathrm{g} / \mathrm{cm}^{3}$

$\mu / \mathrm{mm}^{-1}$

$\mathrm{F}(000)$

Crystal size $/ \mathrm{mm}^{3}$

Radiation

$2 \Theta$ range for data collection $/{ }^{\circ}$

Index ranges

Reflections collected

Independent reflections

Data/restraints/parameters

Goodness-of-fit on $\mathrm{F}^{2}$

Final $\mathrm{R}$ indexes $[\mathrm{I}>=2 \sigma(\mathrm{I})]$

Final $\mathrm{R}$ indexes [all data]

Largest diff. peak/hole / e $\AA^{-3}$
113.194(7)

90

1985.8(2)

4

1.456

0.366

888.0

$0.55 \times 0.35 \times 0.25$

$\operatorname{Mo} \mathrm{K} \alpha(\lambda=0.71073)$

6.618 to 58

$-16 \leq \mathrm{h} \leq 16,-18 \leq \mathrm{k} \leq 16,-16 \leq 1 \leq 15$

15134

$4674\left[R_{\text {int }}=0.0302, R_{\text {sigma }}=0.0408\right]$

$4674 / 0 / 263$

1.036

$\mathrm{R}_{1}=0.0450, \mathrm{wR}_{2}=0.0996$

$\mathrm{R}_{1}=0.0713, \mathrm{wR}_{2}=0.1150$

$0.21 /-0.25$

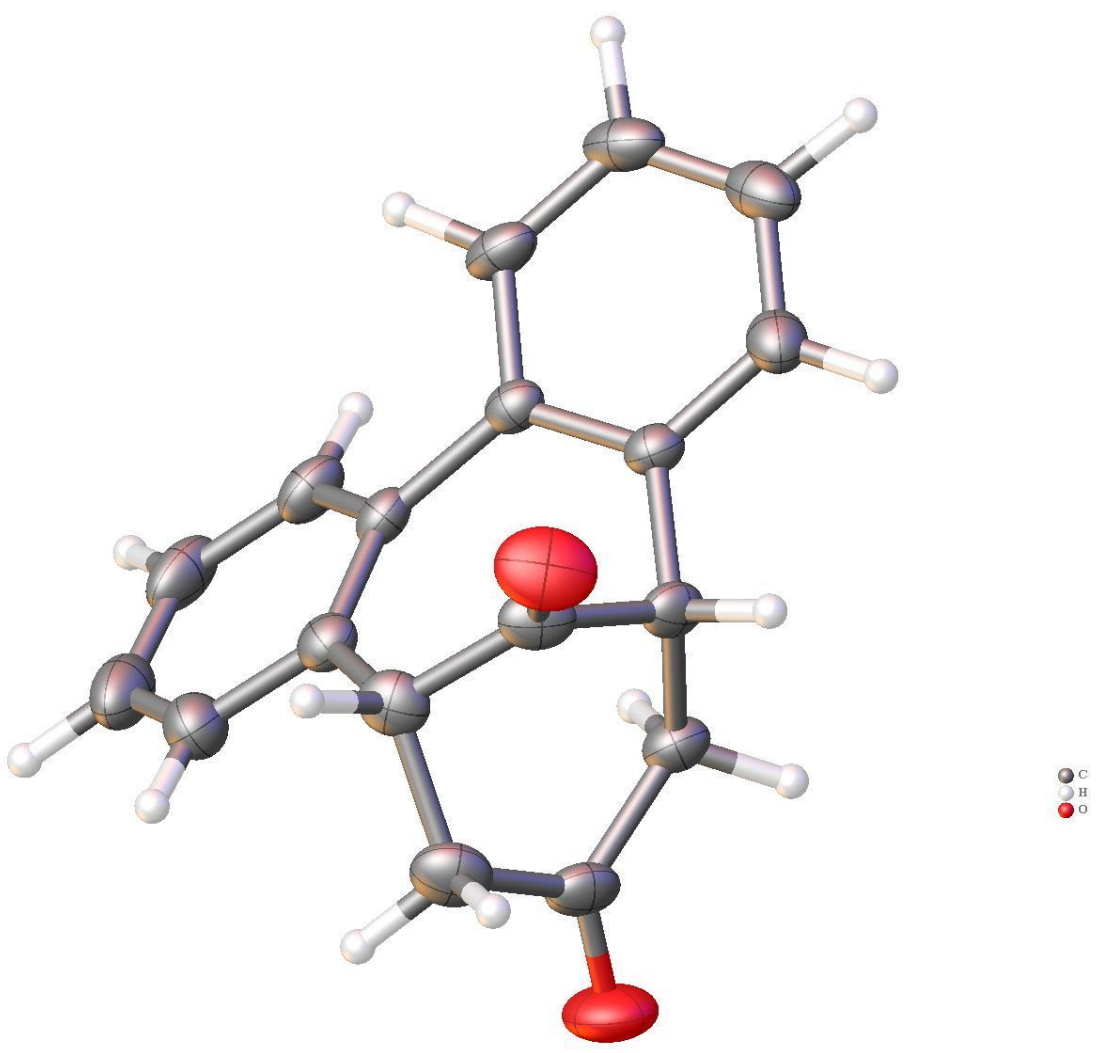

Thermal ellipsoids are set at the 50\% probability level. 
Table S4 Crystal data and structure refinement for 3wa .

Identification code

yly-20200808

Empirical formula

$\mathrm{C}_{18} \mathrm{H}_{14} \mathrm{O}_{2}$

Formula weight

262.29

Temperature/K

296.24(10)

Crystal system

orthorhombic

Space group

Pbca

$\mathrm{a} / \AA$

$11.6068(5)$

$\mathrm{b} / \AA$

$7.2724(3)$

$\mathrm{c} / \AA$

31.0721(13)

$\alpha /{ }^{\circ}$

90

$\beta /^{\circ}$

90

$\gamma /{ }^{\circ}$

90

Volume $/ \AA^{3}$

2622.77(19)

Z

8

$\rho_{\text {calc }} \mathrm{g} / \mathrm{cm}^{3}$

1.329

$\mu / \mathrm{mm}^{-1}$

0.682

$\mathrm{F}(000)$

1104.0

Crystal size $/ \mathrm{mm}^{3}$

$0.1 \times 0.05 \times 0.05$

Radiation

$\mathrm{Cu} \operatorname{K} \alpha(\lambda=1.54184)$

$2 \Theta$ range for data collection ${ }^{\circ}$

9.512 to 142.636

Index ranges

$-10 \leq \mathrm{h} \leq 14,-8 \leq \mathrm{k} \leq 8,-38 \leq 1 \leq 13$

Reflections collected

6518

Independent reflections

$2509\left[\mathrm{R}_{\mathrm{int}}=0.0425, \mathrm{R}_{\text {sigma }}=0.0385\right]$

Data/restraints/parameters

$2509 / 0 / 237$

Goodness-of-fit on $\mathrm{F}^{2}$

1.125

Final R indexes $[\mathrm{I}>=2 \sigma(\mathrm{I})]$

$\mathrm{R}_{1}=0.0727, \mathrm{wR}_{2}=0.2129$

Final $\mathrm{R}$ indexes [all data]

$\mathrm{R}_{1}=0.0847, \mathrm{wR}_{2}=0.2223$

Largest diff. peak/hole / e $\AA^{-3}$

$0.29 /-0.31$ 


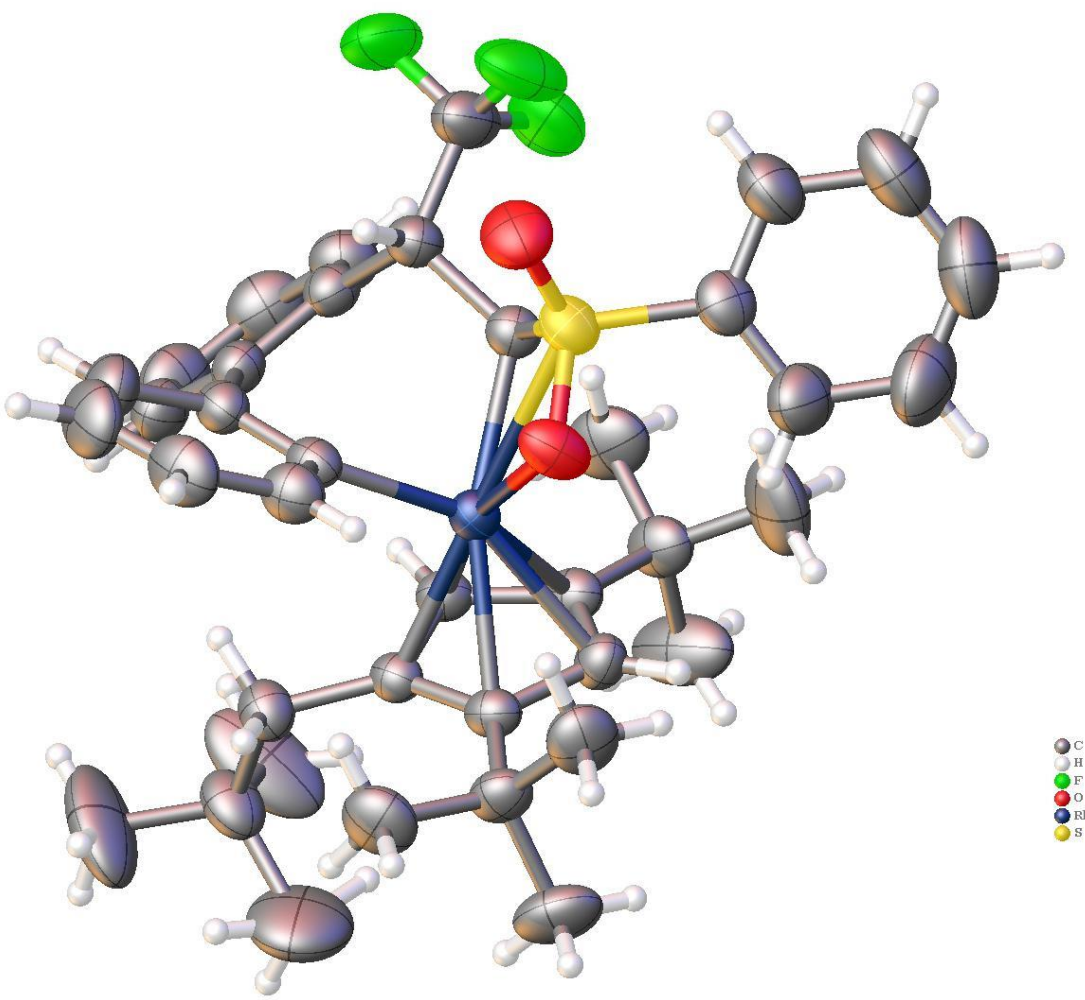

Thermal ellipsoids are set at the $50 \%$ probability level.

Table S5 Crystal data and structure refinement for 3-I.

Identification code

Empirical formula

Formula weight

Temperature/K

Crystal system

Space group

a/Å

$\mathrm{b} / \AA$

c/Å

$\alpha{ }^{\circ}$

$\beta /{ }^{\circ}$

$\gamma /{ }^{\circ}$

Volume $/ \AA^{3}$

Z

$\rho_{\text {calc }} \mathrm{g} / \mathrm{cm}^{3}$

$\mu / \mathrm{mm}^{-1}$

$\mathrm{F}(000)$

Crystal size/ $/ \mathrm{mm}^{3}$

Radiation
YLY-20201106

$\mathrm{C}_{39} \mathrm{H}_{46} \mathrm{~F}_{3} \mathrm{O}_{2} \mathrm{RhS}$

738.73

293(2)

monoclinic

$\mathrm{P} 2{ }_{1} / \mathrm{c}$

9.4589(4)

40.5067(12)

$10.4502(4)$

90

$116.353(5)$

90

3587.9(3)

4

1.368

0.582

1536.0

$0.5 \times 0.3 \times 0.2$

$\operatorname{Mo~K\alpha }(\lambda=0.71073)$ 
$2 \Theta$ range for data collection/ ${ }^{\circ}$

Index ranges

Reflections collected

Independent reflections

Data/restraints/parameters

Goodness-of-fit on $\mathrm{F}^{2}$

Final $\mathrm{R}$ indexes $[\mathrm{I}>=2 \sigma(\mathrm{I})]$

Final R indexes [all data]

Largest diff. peak/hole / e $\AA^{-3}$
6.95 to 57.942

$-11 \leq \mathrm{h} \leq 12,-54 \leq \mathrm{k} \leq 50,-8 \leq$

$1 \leq 14$

16027

$7728\left[\mathrm{R}_{\mathrm{int}}=0.0312, \mathrm{R}_{\mathrm{sigma}}=\right.$

0.0560]

$7728 / 0 / 435$

1.067

$\mathrm{R}_{1}=0.0470, \mathrm{wR}_{2}=0.0819$

$\mathrm{R}_{1}=0.0684, \mathrm{wR}_{2}=0.0911$

$0.34 /-0.48$

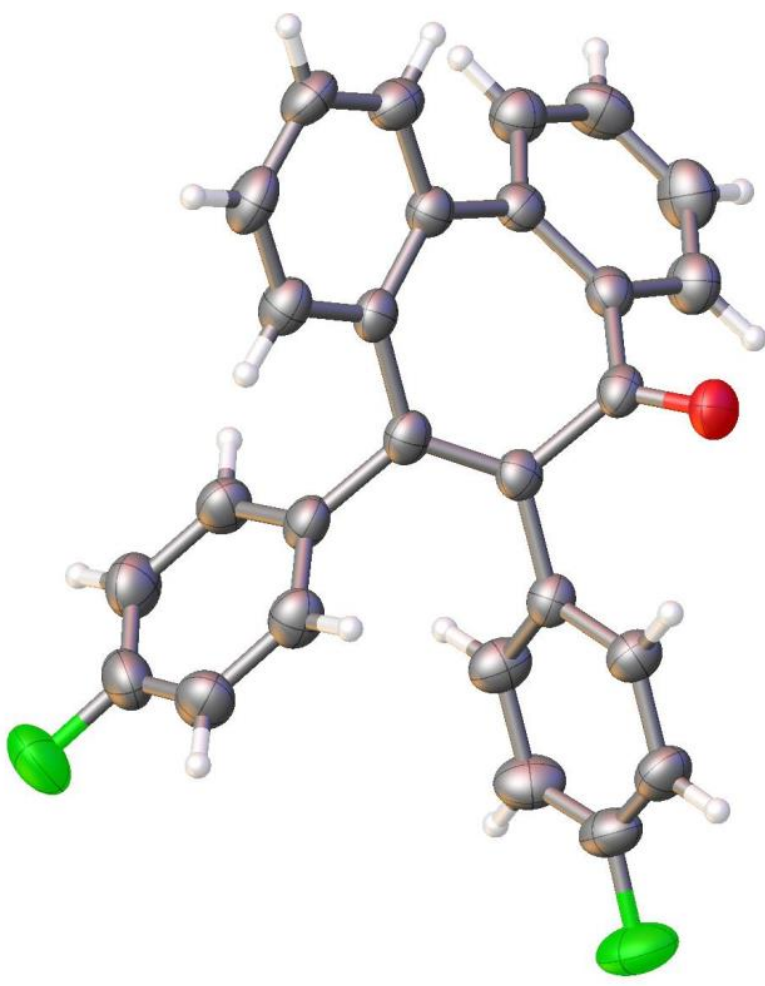

Thermal ellipsoids are set at the $50 \%$ probability level.

Table S6 Crystal data and structure refinement for $5 \mathbf{a b}$.

Identification code

Empirical formula

Formula weight

Temperature/K

Crystal system

Space group

$\mathrm{a} / \AA$

$\mathrm{b} / \AA$
YLY-2-20200825

C27H16F2O

394.40

293(2)

triclinic

P-1

9.2182(14)

10.0895(17) 


\begin{tabular}{|c|c|}
\hline $\mathrm{c} / \AA$ & $10.8958(17)$ \\
\hline$\alpha /{ }^{\circ}$ & $109.297(15)$ \\
\hline$\beta /{ }^{\circ}$ & $95.254(13)$ \\
\hline$\gamma /{ }^{\circ}$ & $93.990(13)$ \\
\hline Volume/Å3 & $947.0(3)$ \\
\hline $\mathrm{Z}$ & 2 \\
\hline pcalcg/cm3 & 1.383 \\
\hline$\mu / \mathrm{mm} 1$ & 0.096 \\
\hline $\mathrm{F}(000)$ & 408.0 \\
\hline Crystal size/mm3 & $0.5 \times 0.3 \times 0.2$ \\
\hline Radiation & $\operatorname{MoK} \alpha(\lambda=0.71073)$ \\
\hline $2 \Theta$ range for data collection $/{ }^{\circ}$ & 6.79 to 58.032 \\
\hline Index ranges & $-12 \leq \mathrm{h} \leq 11,-13 \leq \mathrm{k} \leq 12,-14 \leq 1 \leq 14$ \\
\hline Reflections collected & 11620 \\
\hline Independent reflections & $4428[$ Rint $=0.1020$, Rsigma $=0.0970]$ \\
\hline Data/restraints/parameters & $4428 / 0 / 271$ \\
\hline Goodness-of-fit on F2 & 1.070 \\
\hline Final $R$ indexes $[I>=2 \sigma(I)]$ & $\mathrm{R} 1=0.0850, \mathrm{wR} 2=0.2265$ \\
\hline Final $\mathrm{R}$ indexes [all data] & $\mathrm{R} 1=0.1393, \mathrm{wR} 2=0.3175$ \\
\hline Largest diff. peak/hole / e Å-3 & $0.39 /-0.50$ \\
\hline
\end{tabular}

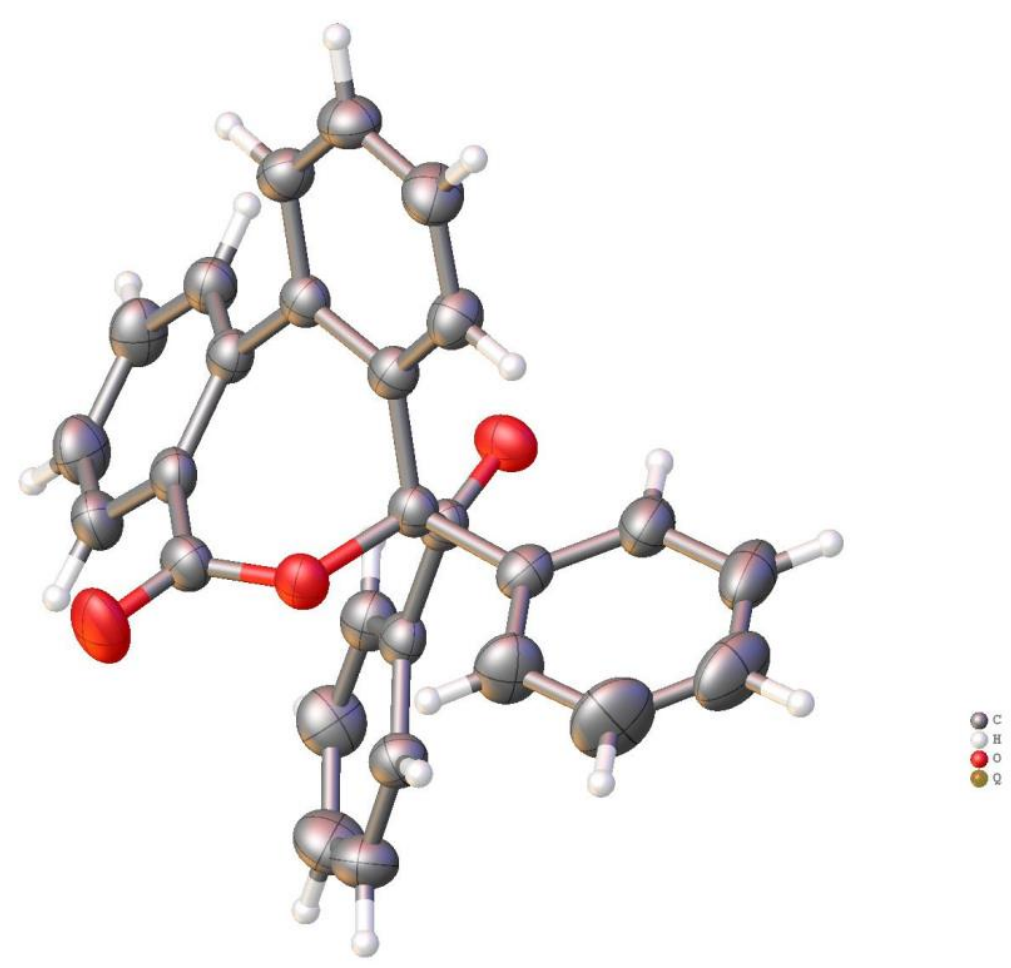

Thermal ellipsoids are set at the $50 \%$ probability level. 
Table S7 Crystal data and structure refinement for 11.

\begin{tabular}{|c|c|}
\hline Identification code & YLY-20210607 \\
\hline Empirical formula & $\mathrm{C} 27 \mathrm{H} 18 \mathrm{O} 3$ \\
\hline Formula weight & 390.41 \\
\hline Temperature/K & 293 \\
\hline Crystal system & monoclinic \\
\hline Space group & $\mathrm{P} 21 / \mathrm{n}$ \\
\hline $\mathrm{a} / \AA$ & $13.0969(2)$ \\
\hline $\mathrm{b} / \AA$ & $11.2557(2)$ \\
\hline $\mathrm{c} / \AA$ & $14.1863(2)$ \\
\hline$\alpha /^{\circ}$ & 90 \\
\hline$\beta /{ }^{\circ}$ & $111.439(2)$ \\
\hline$\gamma /{ }^{\circ}$ & 90 \\
\hline Volume/Å3 & 1946.57(6) \\
\hline $\mathrm{Z}$ & 4 \\
\hline pcalcg/cm3 & 1.332 \\
\hline$\mu / \mathrm{mm} 1$ & 0.689 \\
\hline $\mathrm{F}(000)$ & 816.0 \\
\hline Crystal size/mm3 & $0.5 \times 0.3 \times 0.2$ \\
\hline Radiation & $\mathrm{Cu} \mathrm{K \alpha}(\lambda=1.54184)$ \\
\hline $2 \Theta$ range for data collection $/^{\circ}$ & 10.326 to 142.89 \\
\hline Index ranges & $-15 \leq \mathrm{h} \leq 16,-13 \leq \mathrm{k} \leq 13,-17 \leq 1 \leq 13$ \\
\hline Reflections collected & 8750 \\
\hline Independent reflections & $3723[$ Rint $=0.0175$, Rsigma $=0.0201]$ \\
\hline Data/restraints/parameters & $3723 / 0 / 271$ \\
\hline Goodness-of-fit on F2 & 1.067 \\
\hline Final $R$ indexes $[\mathrm{I}>=2 \sigma(\mathrm{I})]$ & $\mathrm{R} 1=0.0421, \mathrm{wR} 2=0.1069$ \\
\hline Final $\mathrm{R}$ indexes [all data] & $\mathrm{R} 1=0.0461, \mathrm{wR} 2=0.1104$ \\
\hline Largest diff. peak/hole / e $\AA-3$ & $0.17 /-0.25$ \\
\hline
\end{tabular}




\section{References}

1. (a) Suzuki, N.; Fujita, T.; Ichikawa, J. Org. Lett. 2015, 17, 4984-4987. (b) Omann, L.; Oestreich, M. Angew. Chem. Int. Ed. 2015, 54, 10276-10279. (c) Xu, S.-Y.; Huang, B.-L.; Qiao, G.-Y.; Huang, Z.-Y.; Zhang, Z.; Li, Z.-Y.; Wang, P.; Zhang, Z.-H. Org. Lett. 2018, 20, 5578-5582. (d) Zhang, X.; Huang, X.-B.; Gu, W.-X.; Zhang, Y.-B.; Liu, M.-C.; Wu, H.-Y. Adv. Synth. Catal. 2020, 24, 5639-5644. (e) Tokoro, Y.; Oishi, A.; Fukuzawa, S.; Chem. Eur. J. 2016, 22, 13908-13915. (f) Huang, Y.; Liu, L.-J.; Feng, W.-H. ChemistrySelect. 2016, 1, 630-634. (g) Chen, C.-T.; Chao, W.-S.; Liu, H.-W.; Wei, Y.; Jou, J.-H. ; Kumar, S. RSC adv. 2013, 3, 9381-9390.

2. (a) Chai, G.-L.; Sun, A.-Q.; Zhai, D.; Wang, J.; Deng, W.Q.; Wong, H.-C.; Chang, J.-B. Org. Lett. 2019, 21, 5040-5045. (b) Jiang, Q.; Guo, T.; Wu, K.; Yu, Z. Chem. Commun. 2016, 52, 2913-2915; (c) Xiong, H.-Y.; Yang, Z.-Y.; Chen, Z.; Zeng, J.-L.; Nie, J.; Ma, J.-A. Chem. Eur. J. 2014, 20, 8325-8329. (d) Chaudhary, B.; Auti, P.; Shinde, S.D.; Anjaneyulu, P.; Yakkala; Giri, D.; Sharma, S. Org. Lett. 2019, 21, 2763-2767. (e) Jiang, Q.-B.; Guo, T.-L; Yu, Z.-K. J. Org. Chem. 2017, 82, 1951-1960.

3. Bai, D.; Yu. Y.; Guo, H.; Chang, J.; Li, X. Angew. Chem. Int. Ed. 2020, 59, 2740-2744.

4. Trifonova, EA.; Ankudinov, N. M.; Mikhaylov, A. A.; Chusov, D. A.; Nelyubina, Y. V.; Perekalin, D. S. Angew. Chem. Int. Ed. 2018, 57, $7714-7718$.

\section{Characterization Data}

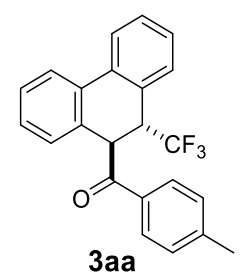

p-Tolyl(10-(trifluoromethyl)-9,10-dihydrophenanthren-9-yl)methanone $(3 \mathrm{aa})$. Eluent: $\mathrm{PE} / \mathrm{EA}=$ 15:1, white solid (34.2 mg, 94\%, m.p. $\left.96-97^{\circ} \mathrm{C}\right) .{ }^{1} \mathrm{H}$ NMR (600 MHz, $\left.\mathrm{CDCl}_{3}\right) \delta 7.80-7.73(\mathrm{~m}, 4 \mathrm{H})$, $7.34-7.31(\mathrm{~m}, 1 \mathrm{H}), 7.28-7.25(\mathrm{~m}, 1 \mathrm{H}), 7.19(\mathrm{~d}, J=8.0 \mathrm{~Hz}, 2 \mathrm{H}), 7.17-7.08(\mathrm{~m}, 4 \mathrm{H}), 5.08(\mathrm{~s}, 1 \mathrm{H})$, $3.84(\mathrm{q}, J=9.4 \mathrm{~Hz}, 1 \mathrm{H}), 2.32(\mathrm{~s}, 3 \mathrm{H}) .{ }^{13} \mathrm{C} \mathrm{NMR}\left(100 \mathrm{MHz}, \mathrm{CDCl}_{3}\right) \delta 196.0,144.6,134.9,134.3,132.6$, $131.3,130.7,129.8,129.7,129.6,128.9,128.7,128.3,128.0,126.4(\mathrm{~d}, J=1.0 \mathrm{~Hz}, 1 \mathrm{C}), 126.3(\mathrm{q}, J=$ $281.8 \mathrm{~Hz}, 1 \mathrm{C}), 124.2,124.1,45.9(\mathrm{~d}, J=1.1 \mathrm{~Hz}, 1 \mathrm{C}), 45.7$ (q, $J=27.3 \mathrm{~Hz}, 1 \mathrm{C}), 21.8 .{ }^{19} \mathrm{~F} \mathrm{NMR}(376$ $\mathrm{MHz}, \mathrm{CDCl}_{3}$ ) $\delta-70.09$ (d). HRMS (ESI) m/z: $[\mathrm{M}+\mathrm{Na}]^{+}$Calcd for $\mathrm{C}_{23} \mathrm{H}_{17} \mathrm{~F}_{3} \mathrm{NaO}$ 389.1124; Found: 389.1124 .

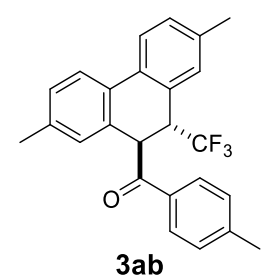

(2,7-Bimethyl-10-(trifluoromethyl)-9,10-dihydrophenanthren-9-yl)(p-tolyl)methanone(3ab).

Eluent: PE/EA = 15:1, white solid $\left(23.0 \mathrm{mg}, 55 \%\right.$, m.p. $\left.114-116{ }^{\circ} \mathrm{C}\right) .{ }^{1} \mathrm{H} \mathrm{NMR}\left(400 \mathrm{MHz}, \mathrm{CDCl}_{3}\right) \delta$ $7.86(\mathrm{~d}, J=8.1 \mathrm{~Hz}, 2 \mathrm{H}), 7.72-7.67(\mathrm{~m}, 2 \mathrm{H}), 7.27$ (d, $J=8.0 \mathrm{~Hz}, 2 \mathrm{H}), 7.18(\mathrm{~d}, J=8.8 \mathrm{~Hz}, 1 \mathrm{H}), 7.13(\mathrm{~d}$, $J=7.8 \mathrm{~Hz}, 1 \mathrm{H}), 6.96(\mathrm{~m}, 2 \mathrm{H}), 5.10(\mathrm{~s}, 1 \mathrm{H}), 3.82(\mathrm{q}, J=9.4 \mathrm{~Hz}, 1 \mathrm{H}), 2.40(\mathrm{~s}, 3 \mathrm{H}), 2.28(\mathrm{~s}, 3 \mathrm{H}), 2.27(\mathrm{~s}$, 
3H). ${ }^{13} \mathrm{C}$ NMR $\left(100 \mathrm{MHz}, \mathrm{CDCl}_{3}\right) \delta 196.1,144.5,137.7,137.4,132.5,132.3,131.9,131.8,130.5$, $130.4,130.1,129.8,129.4,129.0,126.4$ (q, $J=281.9 \mathrm{~Hz}, 1 \mathrm{C}), 125.8,123.7,123.7,45.84,45.83$ (q, $J=$ $27.1 \mathrm{~Hz}, 1 \mathrm{C}), 21.8,21.2,21.1 .{ }^{19} \mathrm{~F}$ NMR $\left(376 \mathrm{MHz}, \mathrm{CDCl}_{3}\right) \delta-70.06(\mathrm{~d}) . \mathrm{HRMS}(\mathrm{ESI}) \mathrm{m} / \mathrm{z}:[\mathrm{M}+\mathrm{Na}]^{+}$ Calcd for $\mathrm{C}_{25} \mathrm{H}_{21} \mathrm{~F}_{3} \mathrm{NaO}$ 417.1437; Found: 417.1437.<smiles>Cc1ccc(C(=O)C2c3cc(Br)ccc3-c3ccc(Br)cc3C2C(C)(F)Br)cc1</smiles>

(2,7-Di-tert-butyl-10-(trifluoromethyl)-9,10-dihydrophenanthren-9-yl)(p-tolyl)methanone(3ac). Eluent: PE/EA = 15:1, white solid $\left(29.3 \mathrm{mg}, 61 \%\right.$, m.p. $\left.105-106{ }^{\circ} \mathrm{C}\right) .{ }^{1} \mathrm{H}$ NMR $\left(400 \mathrm{MHz}, \mathrm{CDCl}_{3}\right) \delta$ $7.88(\mathrm{~d}, J=8.1 \mathrm{~Hz}, 2 \mathrm{H}), 7.77(\mathrm{~d}, J=8.3 \mathrm{~Hz}, 1 \mathrm{H}), 7.74(\mathrm{~d}, J=8.3 \mathrm{~Hz}, 1 \mathrm{H}), 7.43(\mathrm{dd}, J=8.3,1.9 \mathrm{~Hz}$, $1 \mathrm{H}), 7.35(\mathrm{dd}, J=8.2,1.9 \mathrm{~Hz}, 1 \mathrm{H}), 7.31(\mathrm{~d}, J=8.0 \mathrm{~Hz}, 2 \mathrm{H}), 7.19(\mathrm{~s}, 1 \mathrm{H}), 7.13(\mathrm{~d}, J=1.7 \mathrm{~Hz}, 1 \mathrm{H}), 5.14$ (s, 1H), 3.91 (q, $J=9.5 \mathrm{~Hz}, 1 \mathrm{H}), 2.44$ (s, 3H), $1.31(\mathrm{~s}, 9 \mathrm{H}), 1.26(\mathrm{~s}, 9 \mathrm{H}) .{ }^{13} \mathrm{C} \mathrm{NMR}\left(100 \mathrm{MHz}, \mathrm{CDCl}_{3}\right)$ $\delta 196.5,150.8,150.6,144.3,132.9,132.0,131.5,130.0,129.7,129.0,128.3,126.7,126.6,126.5$ (q, $J$ $=281.8 \mathrm{~Hz}, 1 \mathrm{C}), 125.7,125.6,123.5,123.4,45.9$ (q, $J=26.8 \mathrm{~Hz}, 1 \mathrm{C}), 46.5,34.6,31.4,31.3,21.8 .{ }^{19} \mathrm{~F}$ NMR (565 MHz, $\mathrm{CDCl}_{3}$ ) $\delta-70.19$ (d). HRMS (ESI) m/z: [M + Na] ${ }^{+}$Calcd for $\mathrm{C}_{31} \mathrm{H}_{33} \mathrm{~F}_{3} \mathrm{NaO} 501.2376$; Found: 501.2373 .<smiles>COc1ccc(C(=O)C2c3ccc(C)cc3-c3ccc(OC)cc3C2C(=O)c2ccc(C)cc2)cc1</smiles>

(2,7-Dimethoxy-10-(trifluoromethyl)-9,10-dihydrophenanthren-9-yl)(p-tolyl)methanone(3ad). Eluent: PE/EA = 15:1, white solid $\left(25.1 \mathrm{mg}, 29 \%\right.$, m.p. $\left.146-147^{\circ} \mathrm{C}\right) .1 \mathrm{H} \mathrm{NMR}\left(600 \mathrm{MHz}, \mathrm{CDCl}_{3}\right) \delta$ $7.87(\mathrm{~d}, J=8.2 \mathrm{~Hz}, 2 \mathrm{H}), 7.71(\mathrm{~d}, J=8.7 \mathrm{~Hz}, 1 \mathrm{H}), 7.69(\mathrm{~d}, J=8.7 \mathrm{~Hz}, 1 \mathrm{H}), 7.31(\mathrm{~d}, J=8.0 \mathrm{~Hz}, 2 \mathrm{H})$, $6.95(\mathrm{dd}, J=8.7,2.7 \mathrm{~Hz}, 1 \mathrm{H}), 6.88(\mathrm{dd}, J=8.6,2.6 \mathrm{~Hz}, 1 \mathrm{H}), 6.73(\mathrm{~d}, J=2.3 \mathrm{~Hz}, 1 \mathrm{H}), 6.72(\mathrm{~d}, J=2.6$ $\mathrm{Hz}, 1 \mathrm{H}), 5.09$ (s, 1H), 3.85 (q, $J=9.4 \mathrm{~Hz}, 1 \mathrm{H}), 3.79$ (s, 3H), 3.76 (s, 3H), 2.44 (s, 3H). ${ }^{13} \mathrm{C}$ NMR $(100$ $\left.\mathrm{MHz}, \mathrm{CDCl}_{3}\right) \delta 196.0,158.9,158.7,144.6,132.6,131.3,129.8,129.0,127.7,127.3,127.0(\mathrm{~d}, J=0.9$ $\mathrm{Hz}, 1 \mathrm{C}), 126.3$ (q, $J=282.2 \mathrm{~Hz}, 1 \mathrm{C}), 124.7,124.6,116.7,115.19,115.16,113.9,55.42,55.35,46.2$, $46.0(\mathrm{q}, J=26.8 \mathrm{~Hz}, 1 \mathrm{C}), 21.8 .{ }^{19} \mathrm{~F} \mathrm{NMR}\left(376 \mathrm{MHz}, \mathrm{CDCl}_{3}\right) \delta-70.02(\mathrm{~d}) . \mathrm{HRMS}(\mathrm{ESI}) \mathrm{m} / \mathrm{z}:[\mathrm{M}+\mathrm{Na}]^{+}$ Calcd for $\mathrm{C}_{25} \mathrm{H}_{21} \mathrm{~F}_{3} \mathrm{NaO}_{3}$ 449.1335; Found: 449.1331 .<smiles>Cc1ccc(C(=O)C2c3ccc(C)cc3-c3cc(C)ccc3C2C(=O)c2ccccc2)cc1</smiles>

(3,6-Dimethyl-10-(trifluoromethyl)-9,10-dihydrophenanthren-9-yl)(p-tolyl)methanone(3ae). Eluent: PE/EA = 15:1, white solid (32.6 mg, 83\%, m.p. $\left.133-134^{\circ} \mathrm{C}\right) .{ }^{1} \mathrm{H}$ NMR $\left(600 \mathrm{MHz}, \mathrm{CDCl}_{3}\right) \delta$ $7.88(\mathrm{~d}, J=8.2 \mathrm{~Hz}, 2 \mathrm{H}), 7.71(\mathrm{~s}, 1 \mathrm{H}), 7.69(\mathrm{~s}, 1 \mathrm{H}), 7.31(\mathrm{~d}, J=8.0 \mathrm{~Hz}, 2 \mathrm{H}), 7.12-7.05(\mathrm{~m}, 4 \mathrm{H}), 5.15$ 
(s, 1H), 3.90 (q, $J=9.4 \mathrm{~Hz}, 1 \mathrm{H}), 2.44$ (s, 3H), 2.43 (s, 3H), 2.42 (s, 3H). ${ }^{13} \mathrm{C}$ NMR (100 MHz, $\mathrm{CDCl}_{3}$ ) $\delta$ 196.3, 144.4, 139.4, 138.2, 134.7, 134.2, 132.6, 131.1, 129.7, 129.4, 129.0, 128.9, 128.8, 127.8,126.4 $(\mathrm{q}, J=281.7 \mathrm{~Hz}, 1 \mathrm{C}), 124.9,124.8,123.6,45.6,45.4(\mathrm{q}, J=27.1 \mathrm{~Hz}, 1 \mathrm{C}), 21.8,21.7,21.6 .{ }^{19} \mathrm{~F}$ NMR $\left(565 \mathrm{MHz}, \mathrm{CDCl}_{3}\right) \delta-70.23$ (d). HRMS (ESI) m/z: $[\mathrm{M}+\mathrm{Na}]^{+}$Calcd for $\mathrm{C}_{25} \mathrm{H}_{21} \mathrm{~F}_{3} \mathrm{NaO}^{+}$417.1437; Found: 417.1432.<smiles>Cc1ccc(C(=O)C2c3ccc(Cl)cc3-c3cc(Cl)ccc3C2C(F)(F)F)cc1</smiles>

(3,6-Dichloro-10-(trifluoromethyl)-9,10-dihydrophenanthren-9-yl)(p-tolyl)methanone(3af).

Eluent: PE/EA = 15:1, white solid (16.3 mg, 37\%, m.p. $\left.140-141^{\circ} \mathrm{C}\right) .{ }^{1} \mathrm{H}$ NMR $\left(400 \mathrm{MHz}, \mathrm{CDCl}_{3}\right) \delta$ $7.84(\mathrm{~d}, J=8.2 \mathrm{~Hz}, 2 \mathrm{H}), 7.80(\mathrm{~d}, J=2.0 \mathrm{~Hz}, 1 \mathrm{H}), 7.78(\mathrm{~d}, J=2.0 \mathrm{~Hz}, 1 \mathrm{H}), 7.32(\mathrm{~d}, J=8.1 \mathrm{~Hz}, 2 \mathrm{H})$, $7.28-7.23(\mathrm{~m}, 2 \mathrm{H}), 7.17-7.14(\mathrm{~m}, 2 \mathrm{H}), 5.15(\mathrm{~s}, 1 \mathrm{H}), 3.91(\mathrm{q}, J=9.2 \mathrm{~Hz}, 1 \mathrm{H}), 2.45(\mathrm{~s}, 3 \mathrm{H}) .{ }^{13} \mathrm{C}$ NMR $\left(100 \mathrm{MHz}, \mathrm{CDCl}_{3}\right) \delta 195.4,145.1,136.1,135.5,135.0,134.8,132.6,132.1,130.9,130.0,129.3,128.9$, 128.7, 125.9 (q, $J=281.1 \mathrm{~Hz}, 1 \mathrm{C}), 124.9,124.6,124.5,45.23,45.20$ (q, $J=27.8 \mathrm{~Hz}, 1 \mathrm{C}), 21.9 .{ }^{19} \mathrm{~F}$ NMR (376 MHz, $\left.\mathrm{CDCl}_{3}\right) \delta-70.22$ (d). HRMS (ESI) m/z: $[\mathrm{M}+\mathrm{Na}]^{+}$Calcd for $\mathrm{C}_{23} \mathrm{H}_{15} \mathrm{Cl}_{2} \mathrm{~F}_{3} \mathrm{NaO}$ 457.0344; Found:457.0341.<smiles>Cc1ccc(C(=O)C2c3ccc(C(F)(F)F)cc3-c3cc(C(F)(F)F)ccc3C2C(F)(F)F)cc1</smiles>

p-Tolyl(3,6,10-tris(trifluoromethyl)-9,10-dihydrophenanthren-9-yl)methanone(3ag). Eluent: PE/EA = 15:1, white solid $\left(14.4 \mathrm{mg}, 29 \%\right.$, m.p. $\left.130-131{ }^{\circ} \mathrm{C}\right) .{ }^{1} \mathrm{H}$ NMR $\left(600 \mathrm{MHz}, \mathrm{CDCl}_{3}\right) \delta 8.12(\mathrm{~s}$, 2H), 8.09 (s, 1H), $7.85(\mathrm{~d}, J=8.1 \mathrm{~Hz}, 2 \mathrm{H}), 7.58(\mathrm{~d}, J=7.8 \mathrm{~Hz}, 1 \mathrm{H}), 7.55(\mathrm{~d}, J=7.8 \mathrm{~Hz}, 1 \mathrm{H}), 7.41-$ $7.36(\mathrm{~m}, 2 \mathrm{H}), 7.34(\mathrm{~d}, J=8.0 \mathrm{~Hz}, 2 \mathrm{H}), 5.28(\mathrm{~s}, 1 \mathrm{H}), 4.02(\mathrm{q}, J=9.1 \mathrm{~Hz}, 1 \mathrm{H}), 2.45(\mathrm{~s}, 3 \mathrm{H}) .{ }^{13} \mathrm{C} \mathrm{NMR}$ $\left(150 \mathrm{MHz}, \mathrm{CDCl}_{3}\right) \delta 194.8,145.4,134.8,134.5,134.0,132.4$ (q, $\left.J=32.7 \mathrm{~Hz}, 1 \mathrm{C}\right), 132.1,131.8,131.4$ (q, $J=32.6 \mathrm{~Hz}, 1 \mathrm{C}), 130.3,130.2,130.1,129.0,126.7,125.8(\mathrm{q}, J=3.5 \mathrm{~Hz}, 1 \mathrm{C}), 125.6$ (q, $J=3.8 \mathrm{~Hz}$, 1C), $124.0(\mathrm{q}, J=272.4 \mathrm{~Hz}, 1 \mathrm{C}), 123.9(\mathrm{q}, J=273.1 \mathrm{~Hz}, 1 \mathrm{C}), 121.3(\mathrm{q}, J=3.9 \mathrm{~Hz}, 1 \mathrm{C}), 121.2(\mathrm{q}, J=$ $3.9 \mathrm{~Hz}, 1 \mathrm{C}), 45.52,45.52$ (q, $J=27.7 \mathrm{~Hz}, 1 \mathrm{C}), 21.9 .{ }^{19} \mathrm{~F} \mathrm{NMR}\left(376 \mathrm{MHz}, \mathrm{CDCl}_{3}\right) \delta-62.80$ (d), -70.16 (d). HRMS (ESI) m/z: [M + Na] $]^{+}$Calcd for $\mathrm{C}_{25} \mathrm{H}_{15} \mathrm{~F}_{9} \mathrm{NaO}$ 525.0871; Found: 525.0871.<smiles>Cc1ccc(C(=O)C2c3cccc(F)c3-c3c(F)cccc3C2C(C)C)cc1</smiles>

(4,5-Difluoro-10-(trifluoromethyl)-9,10-dihydrophenanthren-9-yl)(p-tolyl)methanone(3ah).

Eluent: PE/EA = 15:1, white solid (25.1 mg, 38\%, m.p. $\left.76-77^{\circ} \mathrm{C}\right) .{ }^{1} \mathrm{H}$ NMR $\left(400 \mathrm{MHz}, \mathrm{CDCl}_{3}\right) \delta 7.72$ $(\mathrm{d}, J=8.2 \mathrm{~Hz}, 2 \mathrm{H}), 7.24-7.19(\mathrm{~m}, 4 \mathrm{H}), 7.13(\mathrm{~d}, J=9.9 \mathrm{~Hz}, 1 \mathrm{H}), 7.08(\mathrm{~d}, J=10.1 \mathrm{~Hz}, 1 \mathrm{H}), 7.04(\mathrm{~m}$, 
$1 \mathrm{H}), 6.97(\mathrm{~d}, J=7.4 \mathrm{~Hz}, 1 \mathrm{H}), 5.07(\mathrm{~s}, 1 \mathrm{H}), 3.93(\mathrm{q}, J=9.2 \mathrm{~Hz}, 1 \mathrm{H}), 2.36(\mathrm{~s}, 3 \mathrm{H}) .{ }^{13} \mathrm{C}$ NMR $(100 \mathrm{MHz}$, $\left.\mathrm{CDCl}_{3}\right) \delta 195.0,159.8(\mathrm{~d}, J=249.3 \mathrm{~Hz}, 1 \mathrm{C}), 159.6(\mathrm{~d}, J=246.8 \mathrm{~Hz}, 1 \mathrm{C}), 144.8,134.5(\mathrm{~d}, J=3.8 \mathrm{~Hz}$, 1C), 132.4, 130.3, 129.94 (d, $J=9.2 \mathrm{~Hz}, 1 \mathrm{C}), 129.85,129.6$ (d, $J=9.0 \mathrm{~Hz}, 1 \mathrm{C}), 128.8,126.5$ (d, $J=$ $2.8 \mathrm{~Hz}, 1 \mathrm{C}), 125.7$ (q, $J=281.6 \mathrm{~Hz}, 1 \mathrm{C}), 124.9$ (d, $J=2.8 \mathrm{~Hz}, 1 \mathrm{C}), 119.7$ (d, $J=15.3 \mathrm{~Hz}, 1 \mathrm{C}), 119.4$ (d, $J=14.9 \mathrm{~Hz}, 1 \mathrm{C}), 117.8(\mathrm{~d}, J=23.5 \mathrm{~Hz}, 1 \mathrm{C}), 116.8(\mathrm{~d}, J=23.7 \mathrm{~Hz}, 1 \mathrm{C}), 47.0,46.3(\mathrm{q}, J=26.0 \mathrm{~Hz}, 1 \mathrm{C})$, 21.8. ${ }^{19} \mathrm{~F}$ NMR (376 MHz, $\mathrm{CDCl}_{3}$ ) $\delta-69.19$ (d), -104.97 - -105.01 (m), -105.25 - -105.30 (m), -105.90 - -105.94 (m), -106.18 - -106.22 (m). HRMS (ESI) m/z: $[\mathrm{M}+\mathrm{Na}]^{+}$Calcd for $\mathrm{C}_{23} \mathrm{H}_{15} \mathrm{~F}_{5} \mathrm{NaO} 425.0935$; Found: 425.0936.<smiles>COc1cccc(OC)c1-c1c(OC)cccc1C(C(=O)c1ccc(C)cc1)C(F)(F)F</smiles>

(4,5-Dimethoxy-10-(trifluoromethyl)-9,10-dihydrophenanthren-9-yl)(p-tolyl)methanone(3ai).Elue nt: PE/EA = 15:1, yellow solid $\left(11.1 \mathrm{mg}, 26 \%\right.$, m.p. $\left.111-112^{\circ} \mathrm{C}\right) .{ }^{1} \mathrm{H}$ NMR $\left(600 \mathrm{MHz}, \mathrm{CDCl}_{3}\right) \delta 7.86$ $(\mathrm{d}, J=8.2 \mathrm{~Hz}, 2 \mathrm{H}), 7.71(\mathrm{~d}, J=8.7 \mathrm{~Hz}, 1 \mathrm{H}), 7.68(\mathrm{~d}, J=8.7 \mathrm{~Hz}, 1 \mathrm{H}), 7.30(\mathrm{~d}, J=8.1 \mathrm{~Hz}, 2 \mathrm{H}), 6.95$ $(\mathrm{dd}, J=8.7,2.6 \mathrm{~Hz}, 1 \mathrm{H}), 6.87(\mathrm{dd}, J=8.6,2.7 \mathrm{~Hz}, 1 \mathrm{H}), 6.72(\mathrm{~d}, J=2.4 \mathrm{~Hz}, 1 \mathrm{H}), 6.71(\mathrm{~d}, J=2.6 \mathrm{~Hz}$, 1H), $5.09(\mathrm{~s}, 1 \mathrm{H}), 3.84(\mathrm{q}, J=9.6 \mathrm{~Hz}, 1 \mathrm{H}), 3.79(\mathrm{~s}, 3 \mathrm{H}), 3.76(\mathrm{~s}, 3 \mathrm{H}), 2.44(\mathrm{~s}, 3 \mathrm{H}) .{ }^{13} \mathrm{C} \mathrm{NMR}(150 \mathrm{MHz}$, $\left.\mathrm{CDCl}_{3}\right) \delta 196.0,158.9,158.7,144.6,132.6,131.3,129.8,129.0,127.9,127.7,127.3,127.0,126.3(\mathrm{q}, J$ $=281.7 \mathrm{~Hz}, 1 \mathrm{C}), 124.7,124.6,116.7,115.19,115.16,114.3,113.9,55.44,55.37,46.2,46.1$ (q, $J=$ $27.1 \mathrm{~Hz}, 1 \mathrm{C}), 21.8 .{ }^{19} \mathrm{~F}$ NMR $\left(376 \mathrm{MHz}, \mathrm{CDCl}_{3}\right) \delta-70.04$ (d). HRMS (ESI) m/z: $[\mathrm{M}+\mathrm{Na}]^{+}$Calcd for $\mathrm{C}_{25} \mathrm{H}_{21} \mathrm{~F}_{3} \mathrm{NaO}_{3} 449.1335$; Found: 449.1330 .<smiles>O=C(c1ccc(F)cc1)C(c1ccccc1)c1ccccc1C(F)(F)F</smiles>

(4-Fluorophenyl)(10-(trifluoromethyl)-9,10-dihydrophenanthren-9-yl)methanone(3ba). Eluent: PE/EA = 15:1, white solid (36.6 mg, 99\%, m.p. $\left.93-94{ }^{\circ} \mathrm{C}\right) .{ }^{1} \mathrm{H}$ NMR $\left(400 \mathrm{MHz}, \mathrm{CDCl}_{3}\right) \delta 7.95-7.91$ (m, 2H), $7.85-7.80(\mathrm{~m}, 2 \mathrm{H}), 7.42-7.38(\mathrm{~m}, 1 \mathrm{H}), 7.36-7.32(\mathrm{~m}, 1 \mathrm{H}), 7.24-7.18(\mathrm{~m}, 3 \mathrm{H}), 7.17-$ $7.10(\mathrm{~m}, 3 \mathrm{H}), 5.09$ (s, 1H), 3.88 (q, $J=9.3 \mathrm{~Hz}, 1 \mathrm{H}) .{ }^{13} \mathrm{C} \mathrm{NMR}\left(100 \mathrm{MHz}, \mathrm{CDCl}_{3}\right) \delta 194.9,166.0(\mathrm{~d}, J$ $=256.0 \mathrm{~Hz}, 1 \mathrm{C}), 134.7,131.6(\mathrm{~d}, J=3.0 \mathrm{~Hz}, 1 \mathrm{C}), 131.5(\mathrm{~d}, J=9.4 \mathrm{~Hz}, 2 \mathrm{C}), 134.3,131.3,130.3,129.9$, 129.6, 128.9, 128.4, 128.2, 126.3 (q, $J=1.0 \mathrm{~Hz}, 1 \mathrm{C}), 126.2$ (q, $J=281.8 \mathrm{~Hz}, 1 \mathrm{C}), 124.3,124.1,116.4$, $116.3(\mathrm{~d}, J=21.9 \mathrm{~Hz}, 2 \mathrm{C}), 116.2,46.1,45.6(\mathrm{q}, J=27.4 \mathrm{~Hz}, 1 \mathrm{C}) .{ }^{19} \mathrm{~F} \mathrm{NMR}\left(376 \mathrm{MHz}, \mathrm{CDCl}_{3}\right) \delta$ -70.13 (d), $-104.17--104.24$ (m). HRMS (ESI) m/z: $[\mathrm{M}+\mathrm{Na}]^{+}$Calcd for $\mathrm{C}_{22} \mathrm{H}_{14} \mathrm{~F}_{4} \mathrm{NaO}$ 393.0873; Found: 393.0872.<smiles>Cc1ccccc1-c1ccccc1C(=O)C(C)c1ccc(Cl)cc1</smiles> 
(4-Chlorophenyl)(10-(trifluoromethyl)-9,10-dihydrophenanthren-9-yl)methanone(3ca).

Eluent:PE/EA = 15:1, white solid (33.5 mg, 87\%, m.p. $\left.120-121{ }^{\circ} \mathrm{C}\right) .{ }^{1} \mathrm{H}$ NMR $\left(400 \mathrm{MHz}, \mathrm{CDCl}_{3}\right) \delta$ $7.93-7.89(\mathrm{~m}, 4 \mathrm{H}), 7.57-7.46(\mathrm{~m}, 3 \mathrm{H}), 7.45-7.41(\mathrm{~m}, 1 \mathrm{H}), 7.35-7.27$ (m, 3H), 7.23 (dd, $J=7.5$, $1.3 \mathrm{~Hz}, 1 \mathrm{H}), 5.16(\mathrm{~s}, 1 \mathrm{H}), 3.97(\mathrm{q}, J=9.4 \mathrm{~Hz}, 1 \mathrm{H}) .{ }^{13} \mathrm{C} \mathrm{NMR}\left(150 \mathrm{MHz}, \mathrm{CDCl}_{3}\right) \delta 195.4,140.1,134.7$, $134.3,133.6,131.3,130.2,130.1,129.9,129.7,129.4,128.9,128.4,128.2,126.4,126.2$ (q, $J=281.7$ $\mathrm{Hz}, 1 \mathrm{C}), 124.3,124.2,46.2$ (s, 1H), 45.5 (q, $J=27.3 \mathrm{~Hz}, 1 \mathrm{C}) .{ }^{19} \mathrm{~F} \mathrm{NMR}\left(376 \mathrm{MHz}, \mathrm{CDCl}_{3}\right) \delta-70.12$ (d). HRMS (ESI) m/z: [M + Na $]^{+}$Calcd for $\mathrm{C}_{22} \mathrm{H}_{14} \mathrm{~F}_{3} \mathrm{ClNaO} 409.0577$; Found: 406.0572.<smiles>O=C(c1ccc(Br)cc1)C1c2ccccc2-c2ccccc2C1C(F)(F)F</smiles>

(4-Bromophenyl)(10-(trifluoromethyl)-9,10-dihydrophenanthren-9-yl)methanone(3da). Eluent: $\mathrm{PE} / \mathrm{EA}=15: 1$, white solid $\left(42.3 \mathrm{mg}, 98 \%\right.$, m.p. $\left.123-125{ }^{\circ} \mathrm{C}\right) .{ }^{1} \mathrm{H} \mathrm{NMR}\left(400 \mathrm{MHz}, \mathrm{CDCl}_{3}\right) \delta 7.77(\mathrm{~m}$, 2H), $7.69(\mathrm{~d}, J=8.6 \mathrm{~Hz}, 2 \mathrm{H}), 7.54(\mathrm{~d}, J=8.6 \mathrm{~Hz}, 2 \mathrm{H}), 7.35-7.33(\mathrm{~m}, 1 \mathrm{H}), 7.287 .31-7.26(\mathrm{~m}, 1 \mathrm{H})$, $7.21-7.13(\mathrm{~m}, 3 \mathrm{H}), 7.09(\mathrm{~d}, J=7.4 \mathrm{~Hz}, 1 \mathrm{H}), 5.01(\mathrm{~s}, 1 \mathrm{H}), 3.83(\mathrm{q}, J=9.3 \mathrm{~Hz}, 1 \mathrm{H}) .{ }^{13} \mathrm{C} \mathrm{NMR}(150$ $\left.\mathrm{MHz}_{\mathrm{CDCl}}\right) \delta 195.6,134.7,134.3,134.1,132.4,131.3,130.2,130.1,129.9,129.7,128.9,128.8$, 128.4, 128.2, 126.4, 126.2 (q, $J=281.6 \mathrm{~Hz}, 1 \mathrm{C}), 124.3,124.1,46.2,45.5$ (q, $J=27.4 \mathrm{~Hz}, 1 \mathrm{C}) .{ }^{19} \mathrm{~F} \mathrm{NMR}$ $\left(376 \mathrm{MHz}, \mathrm{CDCl}_{3}\right) \delta-70.06$ (d). HRMS (ESI) m/z: $[\mathrm{M}+\mathrm{Na}]^{+}$Calcd for $\mathrm{C}_{22} \mathrm{H}_{14} \mathrm{~F}_{3} \mathrm{BrNaO} 453.0072$; Found: 453.0066 .<smiles>COc1ccc(C(=O)C2c3ccccc3-c3ccccc3[C@H]2C(F)(F)F)cc1</smiles>

(4-methoxyphenyl)(10-(trifluoromethyl)-9,10-dihydrophenanthren-9-yl)methanone (3ea). Eluent: $\mathrm{PE} / \mathrm{EA}=15: 1$, white solid $\left(33.9 \mathrm{mg}, 89 \%\right.$, m.p. $\left.165-167{ }^{\circ} \mathrm{C}\right) .{ }^{1} \mathrm{H}$ NMR $\left(600 \mathrm{MHz}, \mathrm{CDCl}_{3}\right) \delta 7.96(\mathrm{~d}, J$ $=8.7 \mathrm{~Hz}, 2 \mathrm{H}), 7.87(\mathrm{~d}, J=7.9 \mathrm{~Hz}, 1 \mathrm{H}), 7.84(\mathrm{~d}, J=7.9 \mathrm{~Hz}, 1 \mathrm{H}), 7.43-7.41(\mathrm{~m}, 1 \mathrm{H}), 7.37-7.34(\mathrm{~m}$, 1H), $7.25-7.21(\mathrm{~m}, 2 \mathrm{H}), 7.19(, 2 \mathrm{H}), 6.97(\mathrm{~d}, J=8.7 \mathrm{~Hz}, 2 \mathrm{H}), 5.15(\mathrm{~s}, 1 \mathrm{H}), 3.92-3.86(\mathrm{~m}, 4 \mathrm{H}) .{ }^{13} \mathrm{C}$ NMR (100 MHz, $\left.\mathrm{CDCl}_{3}\right) \delta 194.8,164.0,134.9,134.4,131.3,131.2,131.0,129.8,129.5,128.7,128.3$, 128.0, 127.8, 126.4, 126.3 (q, $J=281.6 \mathrm{~Hz}, 1 \mathrm{C}), 124.2,124.1,114.3,55.7,45.9$ (q, $J=27.2 \mathrm{~Hz}, 1 \mathrm{C}$ ), 45.5. ${ }^{19} \mathrm{~F}$ NMR (565 MHz, $\left.\mathrm{CDCl}_{3}\right) \delta-70.21$ (d). HRMS (ESI) m/z: $[\mathrm{M}+\mathrm{Na}]^{+}$Calcd for $\mathrm{C}_{23} \mathrm{H}_{17} \mathrm{~F}_{3} \mathrm{NaO}_{2}$ 405.1073; Found: 405.1066.<smiles>O=C(c1ccc(C(F)(F)F)cc1)C1c2ccccc2-c2ccccc2[C@H]1F</smiles>

(10-(Trifluoromethyl)-9,10-dihydrophenanthren-9-yl)(4-(trifluoromethyl)phenyl)methanone(3fa). Eluent: PE/EA = 15:1, white solid $\left(38.5 \mathrm{mg}, 92 \%\right.$, m.p. $\left.95-97^{\circ} \mathrm{C}\right) .{ }^{1} \mathrm{H}$ NMR $\left(400 \mathrm{MHz}, \mathrm{CDCl}_{3}\right) \delta 7.91$ $(\mathrm{d}, J=8.2 \mathrm{~Hz}, 2 \mathrm{H}), 7.84-7.76(\mathrm{~m}, 2 \mathrm{H}), 7.69(\mathrm{~d}, J=8.2 \mathrm{~Hz}, 2 \mathrm{H}), 7.43-7.36(\mathrm{~m}, 1 \mathrm{H}), 7.34-7.31(\mathrm{~m}$, $1 \mathrm{H}), 7.25-7.15(\mathrm{~m}, 3 \mathrm{H}), 7.12(\mathrm{~d}, J=7.4 \mathrm{~Hz}, 1 \mathrm{H}), 5.06(\mathrm{~s}, 1 \mathrm{H}), 3.89(\mathrm{q}, J=9.3 \mathrm{~Hz}, 1 \mathrm{H}) .{ }^{13} \mathrm{C} \mathrm{NMR}$ 
$\left(150 \mathrm{MHz}, \mathrm{CDCl}_{3}\right) \delta 196.0,138.5,134.8(\mathrm{q}, J=33.0 \mathrm{~Hz}, 1 \mathrm{C}), 134.6,134.3,131.3,129.9,129.8,129.6$, 129.1, 129.0, 128.5, 128.3, 126.2 (d, $J=281.9 \mathrm{~Hz}, 1 \mathrm{C}), 126.1$ (q, $J=4.0 \mathrm{~Hz}, 2 \mathrm{C}), 124.4,124.2,123.6$ $(\mathrm{q}, J=272.8 \mathrm{~Hz}, 1 \mathrm{C}), 46.9,45.3(\mathrm{q}, J=27.5 \mathrm{~Hz}, 1 \mathrm{C}) .{ }^{19} \mathrm{~F}$ NMR $\left(376 \mathrm{MHz}, \mathrm{CDCl}_{3}\right) \delta-63.18,-70.04(\mathrm{~d})$. HRMS (ESI) m/z: [M + Na]+ Calcd for $\mathrm{C}_{23} \mathrm{H}_{14} \mathrm{~F}_{6} \mathrm{NaO} 443.0841$; Found: 443.0834<smiles>O=C(c1ccc([N+](=O)[O-])cc1)C1c2ccccc2-c2ccccc2[C@H]1C(F)(F)F</smiles>

(4-Nitrophenyl)(10-(trifluoromethyl)-9,10-dihydrophenanthren-9-yl)methanone(3ga). Eluent: PE/EA $=15: 1$, yellow solid $\left(14.7 \mathrm{mg}, 35 \%\right.$, m.p. $\left.124-126{ }^{\circ} \mathrm{C}\right) .{ }^{1} \mathrm{H}$ NMR $\left(400 \mathrm{MHz}, \mathrm{CDCl}_{3}\right) \delta 8.30(\mathrm{~d}$, $J=8.7 \mathrm{~Hz}, 2 \mathrm{H}), 7.96(\mathrm{~d}, J=8.7 \mathrm{~Hz}, 2 \mathrm{H}), 7.88-7.82(\mathrm{~m}, 2 \mathrm{H}), 7.49-7.44(\mathrm{~m}, 1 \mathrm{H}), 7.41-7.36(\mathrm{~m}$, 1H), $7.34-7.27(\mathrm{~m}, 2 \mathrm{H}), 7.25-7.21(\mathrm{~m}, 1 \mathrm{H}), 7.17(\mathrm{~d}, J=7.5 \mathrm{~Hz}, 1 \mathrm{H}), 5.07(\mathrm{~s}, 1 \mathrm{H}), 3.98$ (q, $J=9.3$ $\mathrm{Hz}, 1 \mathrm{H}) .{ }^{13} \mathrm{C} \mathrm{NMR}\left(100 \mathrm{MHz}, \mathrm{CDCl}_{3}\right) \delta 195.8,150.4,140.8,134.3,134.2,131.3,130.0,129.5,129.3$, $129.0,128.6,128.5,126.5,126.1$ (q, $J=281.5 \mathrm{~Hz}, 1 \mathrm{C}), 124.6,124.2,124.1,47.4(\mathrm{~d}, J=1.2 \mathrm{~Hz}, 1 \mathrm{C})$, $45.1(\mathrm{q}, J=27.5 \mathrm{~Hz}, 1 \mathrm{C}) .{ }^{19} \mathrm{~F}$ NMR $\left(376 \mathrm{MHz}, \mathrm{CDCl}_{3}\right) \delta-69.94$ (d). HRMS (ESI) m/z: $[\mathrm{M}+\mathrm{Na}]^{+}$ Calcd for $\mathrm{C}_{22} \mathrm{H}_{14} \mathrm{~F}_{3} \mathrm{NaO}_{3} 420.0818$; Found: 420.0816 .<smiles>CS(=O)(=O)c1ccc(C(=O)C2c3ccccc3-c3ccccc3[C@@H]2C(F)(F)F)cc1</smiles>

(4-(Methylsulfonyl)phenyl)(10-(trifluoromethyl)-9,10-dihydrophenanthren-9-yl)methanone(3ha). Eluent: PE/EA $=15: 1$, white solid $\left(28.9 \mathrm{mg}, 67 \%\right.$, m.p. $\left.150-151^{\circ} \mathrm{C}\right) .{ }^{1} \mathrm{H} \mathrm{NMR}\left(400 \mathrm{MHz}, \mathrm{CDCl}_{3}\right) \delta$ $8.05(\mathrm{~d}, J=8.5 \mathrm{~Hz}, 2 \mathrm{H}), 7.98(\mathrm{~d}, J=8.5 \mathrm{~Hz}, 2 \mathrm{H}), 7.87-7.83(\mathrm{~m}, 2 \mathrm{H}), 7.48-7.44(\mathrm{~m}, 1 \mathrm{H}), 7.40-7.36$ $(\mathrm{m}, 1 \mathrm{H}), 7.33-7.29(\mathrm{~m}, 2 \mathrm{H}), 7.25-7.21(\mathrm{~m}, 1 \mathrm{H}), 7.18-7.16(\mathrm{~m}, 1 \mathrm{H}), 5.08(\mathrm{~s}, 1 \mathrm{H}), 3.97(\mathrm{q}, J=9.3$ $\mathrm{Hz}, 1 \mathrm{H}), 3.08(\mathrm{~s}, 3 \mathrm{H}) .{ }^{13} \mathrm{C} \mathrm{NMR}\left(100 \mathrm{MHz}, \mathrm{CDCl}_{3}\right) \delta 196.0,144.4,140.2,134.4,134.2,131.3,130.0$, 129.4, 129.2, 129.2, 128.6, 128.4, 128.1, 126.5, 126.1 (q, $J=281.8 \mathrm{~Hz}, 1 \mathrm{C}), 124.5,124.2,47.3,45.1$ (q, $J=27.5 \mathrm{~Hz}, 1 \mathrm{C}), 44.4 .{ }^{19} \mathrm{~F}$ NMR $\left(376 \mathrm{MHz} \mathrm{CDCl}_{3}\right) \delta-69.94(\mathrm{~d}) . \mathrm{HRMS}(\mathrm{ESI}) \mathrm{m} / \mathrm{z}:[\mathrm{M}+\mathrm{Na}]^{+} \mathrm{Calcd}$ for $\mathrm{C}_{23} \mathrm{H}_{17} \mathrm{~F}_{3} \mathrm{NaO}_{3} \mathrm{~S}$ 453.0743; Found: 453.0741 .<smiles>N#Cc1ccccc1C(=O)C1c2ccccc2-c2ccccc21</smiles>

3ia

$o$-Tolyl(10-(trifluoromethyl)-9,10-dihydrophenanthren-9-yl)methanone(3ia). Eluent: PE/EA = 15:1, white solid (18.4 mg, 47\%, m.p. $\left.80-81^{\circ} \mathrm{C}\right) .{ }^{1} \mathrm{H} \mathrm{NMR}\left(600 \mathrm{MHz}, \mathrm{CDCl}_{3}\right) \delta 7.86(\mathrm{~d}, J=7.8 \mathrm{~Hz}, 1 \mathrm{H})$, $7.81(\mathrm{~d}, J=7.7 \mathrm{~Hz}, 1 \mathrm{H}), 7.48-7.43(\mathrm{~m}, 2 \mathrm{H}), 7.39-7.30(\mathrm{~m}, 4 \mathrm{H}), 7.28-7.26(\mathrm{~m}, 1 \mathrm{H}), 7.17-7.15(\mathrm{~m}$, $1 \mathrm{H}), 7.12(\mathrm{~d}, J=7.6 \mathrm{~Hz}, 1 \mathrm{H}), 7.02(\mathrm{~d}, J=7.4 \mathrm{~Hz}, 1 \mathrm{H}), 4.79(\mathrm{~s}, 1 \mathrm{H}), 4.15(\mathrm{q}, J=9.6 \mathrm{~Hz}, 1 \mathrm{H}), 1.81$ (s, 3H). ${ }^{13} \mathrm{C}$ NMR $\left(100 \mathrm{MHz}, \mathrm{CDCl}_{3}\right) \delta 201.5,137.9,137.0,134.4,133.9,131.4,130.6,130.6,129.7$, 128.7, 128.3, 128.2, 127.5 (d, $J=1.1 \mathrm{~Hz}, 1 \mathrm{C}), 126.4$ (q, $J=281.3 \mathrm{~Hz}, 1 \mathrm{C}), 126.2,125.4,124.1,124.0$, 
$50.6(\mathrm{~d}, J=1.1 \mathrm{~Hz}, 1 \mathrm{C}), 44.4(\mathrm{q}, J=27.5 \mathrm{~Hz}, 1 \mathrm{C}), 19.3 .{ }^{19} \mathrm{~F}$ NMR $\left(376 \mathrm{MHz}, \mathrm{CDCl}_{3}\right) \delta-69.80$ (d). HRMS (ESI) m/z: [M + Na] $]^{+}$Calcd for $\mathrm{C}_{23} \mathrm{H}_{17} \mathrm{~F}_{3} \mathrm{NaO}$ 389.1124; Found: 389.1125.<smiles>O=C(c1ccccc1F)C1c2ccccc2-c2ccccc2[C@H]1C(F)(F)F</smiles>

(2-Fluorophenyl)(10-(trifluoromethyl)-9,10-dihydrophenanthren-9-yl)methanone(3ja). Eluent: $\mathrm{PE} / \mathrm{EA}=15: 1$, white solid $\left(33.7 \mathrm{mg}, 91 \%\right.$, m.p. $\left.113-115^{\circ} \mathrm{C}\right) .{ }^{1} \mathrm{H}$ NMR $\left(400 \mathrm{MHz}, \mathrm{CDCl}_{3}\right) \delta 7.95-$ $7.91(\mathrm{~m}, 2 \mathrm{H}), 7.85-7.80(\mathrm{~m}, 2 \mathrm{H}), 7.42-7.38(\mathrm{~m}, 1 \mathrm{H}), 7.36-7.32(\mathrm{~m}, 1 \mathrm{H}), 7.24-7.17$ (m, 3H), 7.15 $-7.11(\mathrm{~m}, 3 \mathrm{H}), 5.09$ (s, 1H), 3.87 (q, $J=9.3 \mathrm{~Hz}, 1 \mathrm{H}) .{ }^{13} \mathrm{C} \mathrm{NMR}\left(150 \mathrm{MHz}, \mathrm{CDCl}_{3}\right) \delta 197.5,195.0$, $166.1(\mathrm{~d}, J=255.6 \mathrm{~Hz}, 1 \mathrm{C}), 134.7,134.3,131.6(\mathrm{~d}, J=3.1 \mathrm{~Hz}, 1 \mathrm{C}), 131.5$ (d, $J=9.6 \mathrm{~Hz}, 1 \mathrm{C}), 131.3$, $130.3,129.9,129.6,128.9,128.4,128.2,126.3,126.2$ (q, $J=281.5 \mathrm{~Hz}, 1 \mathrm{C}), 124.3,124.1,116.3(\mathrm{~d}, J=$ $21.9 \mathrm{~Hz}, 1 \mathrm{C}), 46.1,45.6$ (q, $J=27.4 \mathrm{~Hz}, 1 \mathrm{C}) .{ }^{19} \mathrm{~F}$ NMR $\left(376 \mathrm{MHz}, \mathrm{CDCl}_{3}\right) \delta-70.13(\mathrm{~d}),-104.17-$ -104.24 (m). HRMS (ESI) m/z: [M + Na] ${ }^{+}$Calcd for $\mathrm{C}_{22} \mathrm{H}_{14} \mathrm{~F}_{4} \mathrm{NaO}$ 393.0873; Found: 393.0874 .

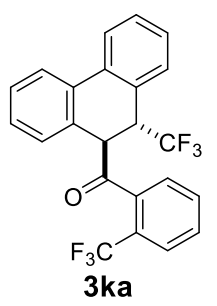

(10-(Trifluoromethyl)-9,10-dihydrophenanthren-9-yl)(2-(trifluoromethyl)phenyl)methanone(3ka). Eluent: PE/EA = 15:1, colorless oily liquid (20.8 mg, 47\%). ${ }^{1} \mathrm{H}$ NMR $\left(400 \mathrm{MHz}, \mathrm{CDCl}_{3}\right) \delta 7.85(\mathrm{~d}, J=$ $7.9 \mathrm{~Hz}, 1 \mathrm{H}), 7.78(\mathrm{~d}, J=7.5 \mathrm{~Hz}, 1 \mathrm{H}), 7.63(\mathrm{~d}, J=7.9 \mathrm{~Hz}, 1 \mathrm{H}), 7.49-7.42(\mathrm{~m}, 3 \mathrm{H}), 7.38-7.34(\mathrm{~m}$, 2H),7.33- $7.28(\mathrm{~m}, 1 \mathrm{H}), 7.08-7.04(\mathrm{~m}, 1 \mathrm{H}), 6.90(\mathrm{~d}, J=7.4 \mathrm{~Hz}, 1 \mathrm{H}), 6.74(\mathrm{~d}, J=7.7 \mathrm{~Hz}, 1 \mathrm{H}), 4.64(\mathrm{~s}$ $1 \mathrm{H}), 4.30(\mathrm{q}, J=9.7 \mathrm{~Hz}, 1 \mathrm{H}) .{ }^{13} \mathrm{C} \mathrm{NMR}\left(100 \mathrm{MHz}, \mathrm{CDCl}_{3}\right) \delta 200.3,137.6(\mathrm{q}, J=2.1 \mathrm{~Hz}, 1 \mathrm{C}), 134.0$, $133.9,131.7,131.3,130.8,130.0,129.7,129.0,128.9,128.5,128.2,127.7$ (q, $J=1.0 \mathrm{~Hz}, 1 \mathrm{C}), 127.3$, 126.9 (q, $J=32.1 \mathrm{~Hz}, 1 \mathrm{C}), 126.6$ (q, $J=4.6 \mathrm{~Hz}, 1 \mathrm{C}), 126.4$ (q, $J=281.2 \mathrm{~Hz}, 1 \mathrm{H}), 124.13,124.11$, $123.6(\mathrm{q}, J=273.8 \mathrm{~Hz}, 1 \mathrm{C}), 51.9,43.7(\mathrm{q}, J=27.6 \mathrm{~Hz}, 1 \mathrm{C}) .{ }^{19} \mathrm{~F}$ NMR $\left(376 \mathrm{MHz}, \mathrm{CDCl}_{3}\right) \delta-57.93$, -69.89 (d). HRMS (ESI) m/z: [M + Na] ${ }^{+}$Calcd for $\mathrm{C}_{23} \mathrm{H}_{14} \mathrm{~F}_{6} \mathrm{NaO} 443.0841$; Found: 443.0844 .<smiles>Cc1cccc(C(=O)C2c3ccccc3-c3ccccc3[C@H]2C(F)(F)F)c1</smiles>

$m$-Tolyl(10-(trifluoromethyl)-9,10-dihydrophenanthren-9-yl)methanone(3la). Eluent: PE/EA = 15:1, white solid (36.2 mg, 99\%, m.p. $\left.90-92{ }^{\circ} \mathrm{C}\right) .{ }^{1} \mathrm{H}$ NMR (400 MHz, $\left.\mathrm{CDCl}_{3}\right) \delta 7.80-7.75$ (m, 2H), $7.68(\mathrm{~d}, J=6.7 \mathrm{~Hz}, 1 \mathrm{H}), 7.62(\mathrm{~s}, 1 \mathrm{H}), 7.37-7.26(\mathrm{~m}, 4 \mathrm{H}), 7.20-7.10(\mathrm{~m}, 4 \mathrm{H}), 5.09(\mathrm{~s}, 1 \mathrm{H}), 3.86(\mathrm{q}, J$ $=9.4 \mathrm{~Hz}, 1 \mathrm{H}), 2.32(\mathrm{~s}, 3 \mathrm{H}) \cdot{ }^{13} \mathrm{C} \mathrm{NMR}\left(100 \mathrm{MHz}, \mathrm{CDCl}_{3}\right) \delta 196.7,139.0,135.3,134.8,134.4,131.3$, 130.6, 129.8, 129.6, 129.4, 128.9, 128.7, 128.3, 128.1, 126.4, 126.3 (q, $J=281.9 \mathrm{~Hz}, 1 \mathrm{C}), 125.9,124.2$, 
124.1, $46.1(\mathrm{~d}, J=1.1 \mathrm{~Hz}, 1 \mathrm{C}), 45.6(\mathrm{q}, J=27.2 \mathrm{~Hz}, 1 \mathrm{C}), 21.5 .{ }^{19} \mathrm{~F}$ NMR $\left(376 \mathrm{MHz}, \mathrm{CDCl}_{3}\right) \delta-70.11$ (d). HRMS (ESI) m/z: [M + Na $]^{+}$Calcd for $\mathrm{C}_{23} \mathrm{H}_{17} \mathrm{~F}_{3} \mathrm{NaO} 389.1124$; Found: 389.1123 .

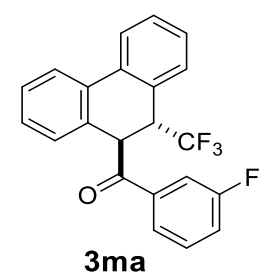

(3-Fluorophenyl)(10-(trifluoromethyl)-9,10-dihydrophenanthren-9-yl)methanone(3ma). Eluent: PE/EA = 15:1, white solid (34.4 mg, 93\%, m.p.72 - $\left.74{ }^{\circ} \mathrm{C}\right) .{ }^{1} \mathrm{H}$ NMR (400 MHz, $\left.\mathrm{CDCl}_{3}\right) \delta 7.89-7.80$ $(\mathrm{m}, 2 \mathrm{H}), 7.76-7.71(\mathrm{~m}, 1 \mathrm{H}), 7.55-7.52(\mathrm{~m}, 1 \mathrm{H}), 7.51-7.40(\mathrm{~m}, 2 \mathrm{H}), 7.39-7.34(\mathrm{~m}, 1 \mathrm{H}), 7.31-$ $7.20(\mathrm{~m}, 4 \mathrm{H}), 7.17(\mathrm{dd}, J=7.6,1.3 \mathrm{~Hz}, 1 \mathrm{H}), 5.09$ (s, 1H), $3.92(\mathrm{q}, J=9.3 \mathrm{~Hz}, 1 \mathrm{H}) .{ }^{13} \mathrm{C} \mathrm{NMR}(150 \mathrm{MHz}$, $\left.\mathrm{CDCl}_{3}\right) \delta 195.4(\mathrm{~d}, J=1.6 \mathrm{~Hz}, 1 \mathrm{C}), 163.1(\mathrm{~d}, J=249.4 \mathrm{~Hz}, 1 \mathrm{C}), 137.5(\mathrm{~d}, J=6.2 \mathrm{~Hz}, 1 \mathrm{C}), 134.7,134.3$, $131.3,130.7$ (d, $J=7.7 \mathrm{~Hz}, 1 \mathrm{C}), 130.0,129.9,129.7,129.0,128.4,128.2,126.2$ (q, $J=281.8 \mathrm{~Hz}, 1 \mathrm{C}$ ), 124.4, 124.3 (d, $J=3.3 \mathrm{~Hz}, 1 \mathrm{C}), 124.2,120.6$ (d, $J=21.7 \mathrm{~Hz}, 1 \mathrm{C}), 115.7$ (d, $J=22.2 \mathrm{~Hz}, 1 \mathrm{C}), 46.5$, 45.4 (q, $J=27.3 \mathrm{~Hz}, 1 \mathrm{C}) .{ }^{19} \mathrm{~F}$ NMR $\left(376 \mathrm{MHz}, \mathrm{CDCl}_{3}\right) \delta-70.07$ (d), -110.66 - -110.73 (m). HRMS (ESI) $\mathrm{m} / \mathrm{z}:[\mathrm{M}+\mathrm{Na}]^{+}$Calcd for $\mathrm{C}_{22} \mathrm{H}_{14} \mathrm{~F}_{4} \mathrm{NaO}$ 393.0873; Found: 393.0873 .<smiles>O=C(c1cccc(Cl)c1)C1c2ccccc2-c2ccccc2C1C(F)(F)F</smiles>

(3-Chlorophenyl)(10-(trifluoromethyl)-9,10-dihydrophenanthren-9-yl)methanone(3na). Eluent: $\mathrm{PE} / \mathrm{EA}=15: 1$, light yellow oily liquid $(27.5 \mathrm{mg}, 71 \%) .{ }^{1} \mathrm{H} \mathrm{NMR}\left(600 \mathrm{MHz}, \mathrm{CDCl}_{3}\right) \delta 7.86-7.83(\mathrm{~m}$, 3H), 7.79 (d, $J=7.8 \mathrm{~Hz}, 1 \mathrm{H}), 7.55(\mathrm{~d}, J=6.3 \mathrm{~Hz}, 1 \mathrm{H}), 7.45-7.41(\mathrm{~m}, 2 \mathrm{H}), 7.38-7.35(\mathrm{~m}, 1 \mathrm{H}), 7.29-$ $7.26(\mathrm{~m}, 1 \mathrm{H}), 7.24-7.22(\mathrm{~m}, 2 \mathrm{H}), 7.17(\mathrm{~d}, J=7.4 \mathrm{~Hz}, 1 \mathrm{H}), 5.08(\mathrm{~s}, 1 \mathrm{H}), 3.92(\mathrm{q}, J=9.3 \mathrm{~Hz}, 1 \mathrm{H}) .{ }^{13} \mathrm{C}$ NMR $\left(100 \mathrm{MHz}, \mathrm{CDCl}_{3}\right) \delta 195.4,137.0,135.5,134.6,134.3,133.5,131.3,130.3,129.9,129.7,129.0$, 128.9, 128.5, 128.2, 126.7, 126.4 (d, $J=1.1 \mathrm{~Hz}, 1 \mathrm{C}), 126.2$ (q, $J=281.8 \mathrm{~Hz}, 1 \mathrm{C}), 124.4,124.2,46.5$ (d, $J=1.1 \mathrm{~Hz}, 1 \mathrm{C}), 45.4(\mathrm{q}, J=27.5 \mathrm{~Hz}, 1 \mathrm{C}) .{ }^{19} \mathrm{~F} \mathrm{NMR}\left(376 \mathrm{MHz}, \mathrm{CDCl}_{3}\right) \delta-70.03$ (d). HRMS (ESI) $\mathrm{m} / \mathrm{z}:[\mathrm{M}+\mathrm{Na}]^{+}$Calcd for $\mathrm{C}_{22} \mathrm{H}_{14} \mathrm{ClF}_{3} \mathrm{NaO} 409.0577$; Found:409.0577.<smiles>O=C(c1cccc(Br)c1)C1c2ccccc2-c2ccccc2C1C(F)(F)F</smiles>

(3-Bromophenyl)(10-(trifluoromethyl)-9,10-dihydrophenanthren-9-yl)methanone(3oa). Eluent: PE/EA = 15:1, white solid $\left(38.0 \mathrm{mg}, 88 \%\right.$, m.p. $\left.130-131^{\circ} \mathrm{C}\right) .{ }^{1} \mathrm{H}$ NMR $\left(400 \mathrm{MHz}, \mathrm{CDCl}_{3}\right) \delta 7.99-$ $7.98(\mathrm{~m}, 1 \mathrm{H}), 7.87-7.83(\mathrm{~m}, 3 \mathrm{H}), 7.71(\mathrm{~d}, J=8.1 \mathrm{~Hz}, 1 \mathrm{H}), 7.46-7.42(\mathrm{~m}, 1 \mathrm{H}), 7.39-7.35(\mathrm{~m}, 2 \mathrm{H})$, $7.30-7.21(\mathrm{~m}, 3 \mathrm{H}), 7.18(\mathrm{~d}, J=7.4 \mathrm{~Hz}, 1 \mathrm{H}), 5.07(\mathrm{~s}, 1 \mathrm{H}), 3.92(\mathrm{q}, J=9.3 \mathrm{~Hz}, 1 \mathrm{H}) .{ }^{13} \mathrm{C} \mathrm{NMR}(10$ $\left.\mathrm{MHz}, \mathrm{CDCl}_{3}\right) \delta 195.3,137.2,136.4,134.6,134.3,131.8,131.3,130.6,129.9,129.84,129.75,129.0$, 128.5, 128.3, 127.1, $126.4(\mathrm{~d}, J=1.1 \mathrm{~Hz}, 1 \mathrm{C}), 126.2(\mathrm{q}, J=281.9 \mathrm{~Hz}, 1 \mathrm{C}), 124.4,124.2,123.5,46.5(\mathrm{~d}$, $J=1.1 \mathrm{~Hz}, 1 \mathrm{C}), 45.4$ (q, $J=27.4 \mathrm{~Hz}, 1 \mathrm{C}) .{ }^{19} \mathrm{~F}$ NMR $\left(376 \mathrm{MHz}, \mathrm{CDCl}_{3}\right.$ ) $\delta-70.14$ (d). HRMS (ESI) $\mathrm{m} / \mathrm{z}:[\mathrm{M}+\mathrm{Na}]^{+}$Calcd for $\mathrm{C}_{22} \mathrm{H}_{14} \mathrm{BrF}_{3} \mathrm{NaO}$ 453.0072; Found: 453.0072 . 


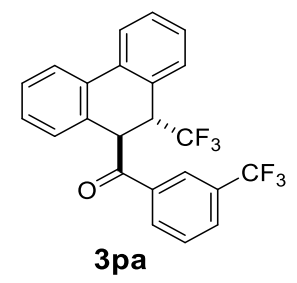

(10-(Trifluoromethyl)-9,10-dihydrophenanthren-9-yl)(3-(trifluoromethyl)phenyl)methanone(3pa). Eluent: PE/EA = 15:1, colorless oily liquid $(32.0 \mathrm{mg}, 76 \%) .{ }^{1} \mathrm{H} \mathrm{NMR}\left(600 \mathrm{MHz}, \mathrm{CDCl}_{3}\right) \delta 8.14(\mathrm{~s}, 1 \mathrm{H})$, $8.10(\mathrm{~d}, J=7.9 \mathrm{~Hz}, 1 \mathrm{H}), 7.88(\mathrm{~d}, J=7.6 \mathrm{~Hz}, 1 \mathrm{H}), 7.87-7.84(\mathrm{~m}, 2 \mathrm{H}), 7.66-7.63(\mathrm{~m}, 1 \mathrm{H}), 7.48-7.45$ (m, 1H), $7.41-7.38(\mathrm{~m}, 1 \mathrm{H}), 7.33-7.30(\mathrm{~m}, 1 \mathrm{H}), 7.29-7.27(\mathrm{~m}, 1 \mathrm{H}), 7.26-7.24(\mathrm{~m}, 1 \mathrm{H}), 7.20(\mathrm{dd}$, $J=7.5,1.1 \mathrm{~Hz}, 1 \mathrm{H}), 5.14(\mathrm{~s}, 1 \mathrm{H}), 3.96(\mathrm{q}, J=9.3 \mathrm{~Hz}, 1 \mathrm{H}) .{ }^{13} \mathrm{C} \mathrm{NMR}\left(150 \mathrm{MHz}, \mathrm{CDCl}_{3}\right) \delta 195.4,136.1$, 134.6, 134.3, 131.73, 131.72 (q, $J=33.1 \mathrm{~Hz}, 1 \mathrm{C}), 131.4,129.91,129.88$ (q, $J=3.4 \mathrm{~Hz}, 1 \mathrm{C}), 129.8$, 129.72, 129.65, 129.1, 128.5, 128.3, 126.4, 126.2 (q, $J=281.7 \mathrm{~Hz}, 1 \mathrm{C}), 125.6$ (q, $J=3.5 \mathrm{~Hz}, 1 \mathrm{C})$, $124.5,124.2,123.6$ (q, $J=272.6 \mathrm{~Hz}, 1 \mathrm{C}), 46.6,45.3$ (q, $J=27.5 \mathrm{~Hz}, 1 \mathrm{C}) .{ }^{19} \mathrm{~F}$ NMR $\left(565 \mathrm{MHz}, \mathrm{CDCl}_{3}\right)$ $\delta$-62.92, -70.07 (d). HRMS (ESI) m/z: [M + Na $]^{+}$Calcd for $\mathrm{C}_{23} \mathrm{H}_{14} \mathrm{~F}_{6} \mathrm{NaO} 443.0841$; Found: 443.0841.<smiles>COc1ccc(C(=O)C2c3ccccc3-c3ccccc3[C@@H]2C(F)(F)F)cc1</smiles>

(3,4-Bimethoxyphenyl)(10-(trifluoromethyl)-9,10-dihydrophenanthren-9-yl)methanone(3qa).Elue nt: PE/EA = 15:1, white solid (30.0 mg, 73\%, m.p. $\left.158-159{ }^{\circ} \mathrm{C}\right) .{ }^{1} \mathrm{H} \mathrm{NMR}\left(600 \mathrm{MHz}, \mathrm{CDCl}_{3}\right) \delta 7.81$ $(\mathrm{d}, J=7.8 \mathrm{~Hz}, 1 \mathrm{H}), 7.79(\mathrm{~d}, J=7.8 \mathrm{~Hz}, 1 \mathrm{H}), 7.68(\mathrm{dd}, J=8.4,1.9 \mathrm{~Hz}, 1 \mathrm{H}), 7.38-7.35(\mathrm{~m}, 1 \mathrm{H}), 7.34$ $(\mathrm{d}, J=1.9 \mathrm{~Hz}, 1 \mathrm{H}), 7.31-7.29(\mathrm{~m}, 1 \mathrm{H}), 7.21-7.17(\mathrm{~m}, 2 \mathrm{H}), 7.17-7.14(\mathrm{~m}, 1 \mathrm{H}), 7.13(\mathrm{~d}, J=7.7 \mathrm{~Hz}$, 1H), $6.90(\mathrm{~d}, J=8.4 \mathrm{~Hz}, 1 \mathrm{H}), 5.11(\mathrm{~s}, 1 \mathrm{H}), 3.90(\mathrm{~s}, 3 \mathrm{H}), 3.87-3.82(\mathrm{~m}, 1 \mathrm{H}), 3.77(\mathrm{~s}, 3 \mathrm{H}) .{ }^{13} \mathrm{C}$ NMR $\left(100 \mathrm{MHz}, \mathrm{CDCl}_{3}\right) \delta 194.9,153.8,149.6,134.9,134.4,131.4,131.0,129.8,129.5,128.7,128.3,128.0$, $127.9,126.4,126.3$ (q, $J=281.8 \mathrm{~Hz}, 1 \mathrm{C}), 124.2,124.1,123.2,111.2,110.3,56.3,56.0,46.0(\mathrm{q}, J=$ $27.2 \mathrm{~Hz}, 1 \mathrm{C}), 45.3$ (s, 1H). ${ }^{19} \mathrm{~F}$ NMR $\left(376 \mathrm{MHz}, \mathrm{CDCl}_{3}\right) \delta-70.22$ (d). HRMS (ESI) m/z: [M + Na] ${ }^{+}$ Calcd for $\mathrm{C}_{24} \mathrm{H}_{19} \mathrm{~F}_{3} \mathrm{NaO}_{3}$ 435.1178; Found: 435.1169 .<smiles>O=C(c1cccc2ccccc12)C1c2ccccc2-c2ccccc21</smiles>

Naphthalen-1-yl(10-(trifluoromethyl)-9,10-dihydrophenanthren-9-yl)methanone(3ra). Eluent: PE/EA = 15:1, white solid (33.4 mg, 83\%, m.p. $\left.114-115^{\circ} \mathrm{C}\right) .{ }^{1} \mathrm{H}$ NMR (400 MHz, $\left.\mathrm{CDCl}_{3}\right) \delta 8.52$ (s, 1H), 7.99 (d, J = 7.8 Hz, 1H), $7.95-7.83(\mathrm{~m}, 5 \mathrm{H}), 7.65-7.54(\mathrm{~m}, 2 \mathrm{H}), 7.45-7.41(\mathrm{~m}, 1 \mathrm{H}), 7.39-$ $7.32(\mathrm{~m}, 1 \mathrm{H}), 7.27-7.19(\mathrm{~m}, 4 \mathrm{H}), 5.34(\mathrm{~s}, 1 \mathrm{H}), 4.01$ (q, $J=9.4 \mathrm{~Hz}, 1 \mathrm{H}) .{ }^{13} \mathrm{C} \mathrm{NMR}\left(100 \mathrm{MHz}, \mathrm{CDCl}_{3}\right)$ $\delta 196.4,135.8,134.8,134.4,132.6,132.5,131.4,130.6,130.4,129.8,129.7,129.1,129.0,128.8,128.4$, 128.1, 128.0, 127.2, 126.5, 126.4 (q, $J=281.7 \mathrm{~Hz}, 1 \mathrm{C}), 124.5,124.3,124.1,46.2,45.8$ (q, $J=27.2 \mathrm{~Hz}$, 1C). ${ }^{19} \mathrm{~F}$ NMR $\left(376 \mathrm{MHz}, \mathrm{CDCl}_{3}\right) \delta-70.09$ (d). HRMS (ESI) m/z: $[\mathrm{M}+\mathrm{Na}]^{+} \mathrm{Calcd}$ for $\mathrm{C}_{26} \mathrm{H}_{17} \mathrm{~F}_{3} \mathrm{NaO}$ 425.1124; Found: 425.1117. 


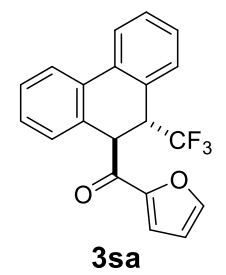

Furan-2-yl(10-(trifluoromethyl)-9,10-dihydrophenanthren-9-yl)methanone(3sa). Eluent: PE/EA = 15:1, yellow oily liquid (33.8 mg, 99\%). ${ }^{1} \mathrm{H} \mathrm{NMR}\left(600 \mathrm{MHz}, \mathrm{CDCl}_{3}\right) \delta 7.88-7.82(\mathrm{~m}, 2 \mathrm{H}), 7.66(\mathrm{~d}, J$ $=1.1 \mathrm{~Hz}, 1 \mathrm{H}), 7.45-7.37(\mathrm{~m}, 3 \mathrm{H}), 7.34-7.26(\mathrm{~m}, 3 \mathrm{H}), 7.11(\mathrm{~d}, J=3.5 \mathrm{~Hz}, 1 \mathrm{H}), 6.54(\mathrm{dd}, J=3.6,1.7$ $\mathrm{Hz}, 1 \mathrm{H}), 4.98(\mathrm{~s}, 1 \mathrm{H}), 4.00(\mathrm{q}, J=9.4 \mathrm{~Hz}, 1 \mathrm{H}) .{ }^{13} \mathrm{C} \mathrm{NMR}\left(100 \mathrm{MHz}, \mathrm{CDCl}_{3}\right) \delta 185.1,151.2,146.9$, 134.6, 134.3, 131.3, , 130.01, 129.95, 129.6, 128.9, 128.3, 128.2, 127.2 (d, $J=1.0 \mathrm{~Hz}, 1 \mathrm{C}), 126.2$ (q, $J$ $=281.5 \mathrm{~Hz}, 1 \mathrm{C}), 124.3,124.1,118.9,113.0,46.8,45.2(\mathrm{q}, J=27.4 \mathrm{~Hz}, 1 \mathrm{C}) .{ }^{19} \mathrm{~F} \mathrm{NMR}(376 \mathrm{MHz}$, $\left.\mathrm{CDCl}_{3}\right) \delta-70.11(\mathrm{~d})$. HRMS (ESI) m/z: $[\mathrm{M}+\mathrm{Na}]^{+}$Calcd for $\mathrm{C}_{20} \mathrm{H}_{13} \mathrm{~F}_{3} \mathrm{NaO}_{2}$ 365.0760; Found:365.0760.<smiles>O=C1CCCC2c3ccccc3-c3ccccc3C12</smiles>

3,4,4a,12b-Tetrahydrotriphenylen-1(2H)-one(3ta). Eluent: $\mathrm{PE} / \mathrm{EA}=15: 1$, yellow oily liquid $(4.0 \mathrm{mg}$, 16\%). ${ }^{1} \mathrm{H} \mathrm{NMR}\left(400 \mathrm{MHz}, \mathrm{CDCl}_{3}\right) \delta 7.84(\mathrm{~d}, J=7.6 \mathrm{~Hz}, 1 \mathrm{H}), 7.79(\mathrm{~d}, J=7.6 \mathrm{~Hz}, 1 \mathrm{H}), 7.41-7.20(\mathrm{~m}$, $5 \mathrm{H}), 6.98(\mathrm{~d}, J=7.6 \mathrm{~Hz}, 1 \mathrm{H}), 3.93(\mathrm{~d}, J=5.6 \mathrm{~Hz}, 1 \mathrm{H}), 3.34-3.25(\mathrm{~m}, 1 \mathrm{H}), 2.60-2.55(\mathrm{~m}, 1 \mathrm{H}), 2.46-$ $2.42(\mathrm{~m}, 1 \mathrm{H}), 2.04-1.99(\mathrm{~m}, 1 \mathrm{H}), 1.94-1.82(\mathrm{~m}, 2 \mathrm{H}), 1.81-1.75(\mathrm{~m}, 1 \mathrm{H}) .{ }^{13} \mathrm{C}$ NMR $(100 \mathrm{MHz}$, $\left.\mathrm{CDCl}_{3}\right) \delta 212.6,138.0,133.6,133.0,132.5,128.4,128.3,128.0,127.8,127.7,127.6,124.3,124.1,54.7$, 43.7, 39.2, 28.1, 25.6. HRMS (ESI) m/z: $[\mathrm{M}+\mathrm{Na}]^{+}$Calcd for $\mathrm{C}_{18} \mathrm{H}_{16} \mathrm{NaO}$ 271.1093; Found: 271.1093.<smiles></smiles>

2-Methyl-3a,11b-dihydro-1H-dibenzo[e,g]isoindole-1,3(2H)-dione(3ua). Eluent: PE/EA = 15:1, white solid (7.8 mg, 30\%, m.p. $\left.166-167^{\circ} \mathrm{C}\right) .{ }^{1} \mathrm{H}$ NMR (600 MHz, $\left.\mathrm{CDCl}_{3}\right) \delta 7.92(\mathrm{~d}, J=7.8 \mathrm{~Hz}, 2 \mathrm{H})$, $7.74(\mathrm{~d}, J=7.4 \mathrm{~Hz}, 2 \mathrm{H}), 7.43-7.33(\mathrm{~m}, 4 \mathrm{H}), 4.36(\mathrm{~s}, 2 \mathrm{H}), 3.00(\mathrm{~s}, 3 \mathrm{H}) .{ }^{13} \mathrm{C} \mathrm{NMR}\left(150 \mathrm{MHz}, \mathrm{CDCl}_{3}\right) \delta$ 177.3, 131.2, 130.1, 128.8, 128.5, 127.0, 123.5, 43.4, 25.7. HRMS (ESI) m/z: $[\mathrm{M}+\mathrm{Na}]^{+}$Calcd for $\mathrm{C}_{17} \mathrm{H}_{13} \mathrm{NNaO}_{2}$ 286.0838; Found: 286.0830 .<smiles>CCC(=O)/C=C/c1ccccc1-c1ccccc1</smiles>

1-([1,1'-Biphenyl]-2-yl)pent-1-en-3-one(3va). Eluent: PE/EA = 15:1, colorless oily liquid (17.3 mg, 73\%). ${ }^{1} \mathrm{H}$ NMR (400 MHz, $\left.\mathrm{CDCl}_{3}\right) \delta 7.71(\mathrm{~d}, J=7.6 \mathrm{~Hz}, 1 \mathrm{H}), 7.58(\mathrm{~d}, J=16.3 \mathrm{~Hz}, 1 \mathrm{H}), 7.47-7.38$ $(\mathrm{m}, 6 \mathrm{H}), 7.35-7.30(\mathrm{~m}, 2 \mathrm{H}), 6.67(\mathrm{~d}, J=16.3 \mathrm{~Hz}, 1 \mathrm{H}), 2.56(\mathrm{q}, J=7.3 \mathrm{~Hz}, 2 \mathrm{H}), 1.10(\mathrm{t}, J=7.3 \mathrm{~Hz}$, $3 \mathrm{H}) .{ }^{13} \mathrm{C} \mathrm{NMR}\left(150 \mathrm{MHz}, \mathrm{CDCl}_{3}\right) \delta 201.3,143.2,141.8,140.1,132.9,130.6,130.1,130.0,128.4$, 127.9, 127.8, 127.6, 126.9, 33.4, 8.3. HRMS (ESI) m/z: $[\mathrm{M}+\mathrm{Na}]^{+}$Calcd for $\mathrm{C}_{17} \mathrm{H}_{16} \mathrm{NaO} 259.1093$; Found: 259.1093. 


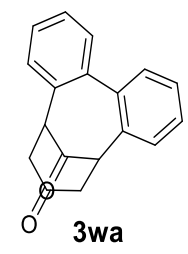

5,6,8,9-Tetrahydro-7H-5,9-methanodibenzo[a,c][9] annulene-7,14-dione (3wa). Eluent: PE/EA = 15:1, white solid (24.3 mg, 93\%, m.p. $\left.133-135{ }^{\circ} \mathrm{C}\right) .{ }^{1} \mathrm{H}$ NMR $\left(600 \mathrm{MHz}, \mathrm{CDCl}_{3}\right) \delta 7.66-7.61(\mathrm{~m}$, 2H), $7.43-7.39(\mathrm{~m}, 4 \mathrm{H}), 7.31-7.27(\mathrm{~m}, 2 \mathrm{H}), 4.12(\mathrm{t}, J=6.0 \mathrm{~Hz}, 2 \mathrm{H}), 3.15(\mathrm{dd}, J=18.6,5.3 \mathrm{~Hz}, 2 \mathrm{H})$, $3.00(\mathrm{dd}, J=18.9,6.9 \mathrm{~Hz}, 2 \mathrm{H}) .{ }^{13} \mathrm{C} \mathrm{NMR}\left(150 \mathrm{MHz}, \mathrm{CDCl}_{3}\right) \delta 205.8,203.5,137.3,135.0,132.5,129.7$, 129.1, 128.5, 53.4, 46.8. HRMS (ESI) $\mathrm{m} / \mathrm{z}$ : $[\mathrm{M}+\mathrm{Na}]^{+}$Calcd for $\mathrm{C}_{18} \mathrm{H}_{14} \mathrm{NaO}_{2}$ 285.0886; Found: 285.0881 .

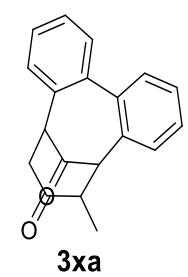

6-Methyl-5,6,8,9-tetrahydro-7H-5,9-methanodibenzo[a,c][9]annulene-7,14-dione(3xa). Eluent: PE/EA = 15:1,yellow solid $\left(17.8 \mathrm{mg}, 64 \%\right.$, m.p. $\left.139-140{ }^{\circ} \mathrm{C}\right) .{ }^{1} \mathrm{H}$ NMR $\left(400 \mathrm{MHz}, \mathrm{CDCl}_{3}\right) \delta 7.69-$ $7.61(\mathrm{~m}, 2 \mathrm{H}), 7.45-7.38(\mathrm{~m}, 4 \mathrm{H}), 7.34-7.26(\mathrm{~m}, 2 \mathrm{H}), 4.18-4.17(\mathrm{~m}, 1 \mathrm{H}), 3.54(\mathrm{~d}, J=8.0 \mathrm{~Hz}, 1 \mathrm{H})$, $3.25(\mathrm{dd}, J=19.1,3.1 \mathrm{~Hz}, 1 \mathrm{H}), 3.21-3.13(\mathrm{~m}, 1 \mathrm{H}), 3.03(\mathrm{dd}, J=19.1,6.4 \mathrm{~Hz}, 1 \mathrm{H}), 1.13(\mathrm{~d}, J=6.8$ $\mathrm{Hz}, 3 \mathrm{H}) .{ }^{13} \mathrm{C}$ NMR $\left(150 \mathrm{MHz}, \mathrm{CDCl}_{3}\right) \delta 207.7,203.4,137.4,137.0,136.0,134.3,132.6,132.3,130.2$, 129.7, 129.3, 128.8, 128.7, 128.1, 62.0, 54.0, 48.7, 47.7, 14.3. HRMS (ESI) m/z: $[\mathrm{M}+\mathrm{Na}]^{+}$Calcd for $\mathrm{C}_{19} \mathrm{H}_{16} \mathrm{NaO}_{2} 299.1043$; Found: 299.1038.<smiles>O=C1C=C(Br)C(=O)c2c1c1ccccc1c1ccccc21</smiles>

2-(Tert-butyl)triphenylene-1,4-dione(3ya). Eluent: PE/EA = 15:1, yellow solid (7.9 mg, 25\%, m.p. $\left.136-137{ }^{\circ} \mathrm{C}\right) .{ }^{1} \mathrm{H}$ NMR $\left(400 \mathrm{MHz}, \mathrm{CDCl}_{3}\right) \delta 9.46-9.35(\mathrm{~m}, 1 \mathrm{H}), 8.89(\mathrm{~d}, J=8.4 \mathrm{~Hz}, 1 \mathrm{H}), 8.74-8.70$ $(\mathrm{m}, 2 \mathrm{H}), 7.81-7.69(\mathrm{~m}, 4 \mathrm{H}), 6.78(\mathrm{~s}, 1 \mathrm{H}), 1.45(\mathrm{~s}, 9 \mathrm{H}) .{ }^{13} \mathrm{C} \mathrm{NMR}\left(150 \mathrm{MHz}, \mathrm{CDCl}_{3}\right) \delta 190.7,189.2$, $158.5,134.3,133.5,133.0,132.8,129.5,129.22$, 129.17, 128.7, 128.4, 128.1, 127.0, 126.8, 123.0, 122.8, 35.6, 29.6. HRMS (ESI) m/z: $[\mathrm{M}+\mathrm{Na}]^{+}$Calcd for $\mathrm{C}_{22} \mathrm{H}_{18} \mathrm{NaO}_{2}$ 337.1199; Found: 337.1196.<smiles>O=c1c(-c2ccccc2)c(-c2ccccc2)c2ccccc2c2ccccc12</smiles>

6,7-Diphenyl-5H-dibenzo[a,c][7]annulen-5-one(5aa). Eluent: $\mathrm{PE} / \mathrm{DCM}=1: 1$, white solid (32.2 mg, $90 \%$, m.p. $\left.150-151^{\circ} \mathrm{C}\right) .{ }^{1} \mathrm{H}$ NMR $\left(600 \mathrm{MHz}, \mathrm{CDCl}_{3}\right) \delta 7.88(\mathrm{~d}, J=7.8 \mathrm{~Hz}, 1 \mathrm{H}), 7.72$ (dd, $J=8.0,1.1$ $\mathrm{Hz}, 1 \mathrm{H}), 7.65-7.63$ (m, 1H), $7.45-7.43$ (m, 1H), $7.40-7.37$ (m, 1H), 7.34 (dd, J = 7.6, 1.2 Hz, 1H), 
$7.26-7.20(\mathrm{~m}, 3 \mathrm{H}), 7.14-7.08(\mathrm{~m}, 7 \mathrm{H}), 6.99-6.96(\mathrm{~m}, 2 \mathrm{H}) .{ }^{13} \mathrm{C} \mathrm{NMR}\left(150 \mathrm{MHz}, \mathrm{CDCl}_{3}\right)$ 8197.9, 144.2, 142.6, 141.8, 141.1, 137.8, 137.2, 136.4, 136.1, 134.2, 133.8, 131.7, 131.4, 131.0, 130.8, 130.1, 129.4, 129.2, 129.1, 128.9, 128.7, 128.5, 128.4, 127.8, 127.7, 127.6, 125.7. HRMS (ESI) m/z: [M + $\mathrm{Na}]^{+}$Calcd for $\mathrm{C}_{27} \mathrm{H}_{18} \mathrm{NaO} 381.1250$; Found: 381.1245 .

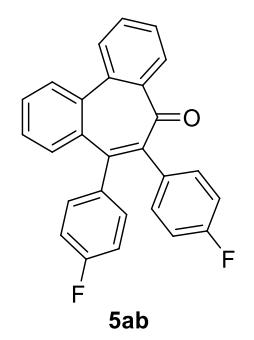

6,7-Bis(4-fluorophenyl)-5H-dibenzo[a,c][7]annulen-5-one(5ab). Eluent: $\mathrm{PE} / \mathrm{DCM}=1: 1$, white solid (24.5 mg, 62\%, m.p. $\left.224-225^{\circ} \mathrm{C}\right) .{ }^{1} \mathrm{H}$ NMR $\left(600 \mathrm{MHz}, \mathrm{CDCl}_{3}\right) \delta 7.90(\mathrm{~d}, J=7.9 \mathrm{~Hz}, 1 \mathrm{H}), 7.74(\mathrm{dd}, J$ $=7.9,0.9 \mathrm{~Hz}, 1 \mathrm{H}), 7.69-7.66(\mathrm{~m}, 1 \mathrm{H}), 7.49-7.46(\mathrm{~m}, 1 \mathrm{H}), 7.45-7.41(\mathrm{~m}, 1 \mathrm{H}), 7.35(\mathrm{dd}, J=7.6,1.0$ $\mathrm{Hz}, 1 \mathrm{H}), 7.28-7.20(\mathrm{~m}, 3 \mathrm{H}), 7.10(\mathrm{dd}, J=8.0,1.0 \mathrm{~Hz}, 1 \mathrm{H}), 6.98-6.91(\mathrm{~m}, 2 \mathrm{H}), 6.90-6.78(\mathrm{~m}, 4 \mathrm{H})$. ${ }^{13} \mathrm{C}$ NMR $\left(150 \mathrm{MHz}, \mathrm{CDCl}_{3}\right) \delta 198.7,162.1(\mathrm{~d}, J=247.3 \mathrm{~Hz}, 1 \mathrm{C}), 161.9(\mathrm{~d}, J=247.9 \mathrm{~Hz}, 1 \mathrm{C}), 144.4$, 141.9, 141.0, 137.7, 137.1 (d, $J=3.7 \mathrm{~Hz}, 1 \mathrm{C}), 136.8,136.4,132.8$ (d, $J=7.9 \mathrm{~Hz}, 1 \mathrm{C}), 132.0$ (d, $J=7.9$ $\mathrm{Hz}, 1 \mathrm{C}), 131.8,131.7$ (d, $J=3.3 \mathrm{~Hz}, 1 \mathrm{C}), 131.2,130.9,128.8,128.4$ (d, $J=7.5 \mathrm{~Hz}, 1 \mathrm{C}), 127.4,125.6$, $115.2(\mathrm{~d}, J=21.8 \mathrm{~Hz}, 1 \mathrm{C}), 114.9(\mathrm{~d}, J=21.1 \mathrm{~Hz}, 1 \mathrm{C}) .{ }^{19} \mathrm{~F}$ NMR $\left(376 \mathrm{MHz}, \mathrm{CDCl}_{3}\right) \delta-114.15-$ $-114.22(\mathrm{~m}),-114.44--114.51(\mathrm{~m})$. HRMS (ESI) m/z: $[\mathrm{M}+\mathrm{Na}]^{+} \mathrm{Calcd}$ for $\mathrm{C}_{27} \mathrm{H}_{16} \mathrm{~F}_{2} \mathrm{NaO} 417.1563$; Found: 417.1561.

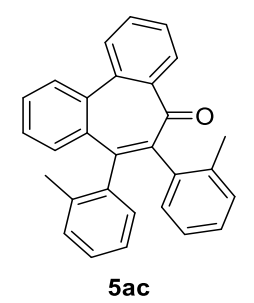

6,7-Di-o-tolyl-5H-dibenzo[a,c][7]annulen-5-one(5ac). Eluent: $\mathrm{PE} / \mathrm{DCM}=1: 1$, white solid (30.8 mg, 80\%). ${ }^{1} \mathrm{H}$ NMR $\left(600 \mathrm{MHz}, \mathrm{CDCl}_{3}\right)$ for the diastereoisomers $\delta 7.89(\mathrm{~m}, 2 \mathrm{H}), 7.80-7.77(\mathrm{~m}, 1 \mathrm{H}), 7.75-$ $7.74(\mathrm{~m}, 1 \mathrm{H}), 7.70-7.68(\mathrm{~m}, 3 \mathrm{H}), 7.53-7.50(\mathrm{~m}, 2 \mathrm{H}), 7.48-7.40(\mathrm{~m}, 4 \mathrm{H}), 7.29-7.17(\mathrm{~m}, 5 \mathrm{H}), 7.14$ $-7.00(\mathrm{~m}, 8 \mathrm{H}), 7.00-6.91(\mathrm{~m}, 4 \mathrm{H}), 6.87-6.80(\mathrm{~m}, 2 \mathrm{H}), 6.74(\mathrm{~s}, 1 \mathrm{H}), 2.50(\mathrm{~s}, 2.5 \mathrm{H}), 1.85(\mathrm{~s}, 2.5 \mathrm{H})$, $1.67(\mathrm{~s}, 3 \mathrm{H}), 1.65(\mathrm{~s}, 3 \mathrm{H}) .{ }^{13} \mathrm{C} \mathrm{NMR}\left(150 \mathrm{MHz}, \mathrm{CDCl}_{3}\right)$ for the diastereoisomers $\delta 198.3,197.6,171.3$, 145.1, 144.3, 143.8, 143.3, 140.2, 139.3, 137.7, 137.4, 137.3, 137.0, 136.4, 136.2, 136.1, 135.7, 135.6, 135.0, 132.8, 132.0, 131.2, 131.1, 130.8, 130.7, 130.6, 130.2, 130.1, 130.0, 129.6, 129.0, 128.6, 128.5, 128.4, 128.1, 128.0, 127.7, 127.6, 127.4, 126.5, 125.8, 125.2, 125.0, 124.8, 60.5, 21.3, 21.2, 19.9, 19.7, 14.4. HRMS (ESI) m/z: $[\mathrm{M}+\mathrm{Na}]^{+}$Calcd for $\mathrm{C}_{29} \mathrm{H}_{22} \mathrm{NaO}$ 409.1563; Found: 409.1561 .

Note: 5ac could not be separated by silica gel column chromatography. ${ }^{1} \mathrm{H}$ NMR spectra and ${ }^{13} \mathrm{C}$ NMR pectra of the mixture were provided. 


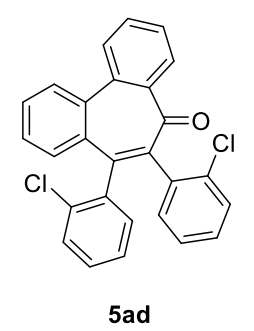

6,7-Bis(2-chlorophenyl)-5H-dibenzo[a,c][7]annulen-5-one(5ad). Eluent: PE/DCM = 1:1, colorless liquid (33.6 mg, 79\%). ${ }^{1} \mathrm{H} \mathrm{NMR} \mathrm{(600} \mathrm{MHz,} \mathrm{CDCl}_{3}$ ) for the diastereoisomers $\delta 7.90-7.89(\mathrm{~m}, 1 \mathrm{H}), 7.81$ $-7.74(\mathrm{~m}, 2 \mathrm{H}), 7.74-7.70(\mathrm{~m}, 1 \mathrm{H}), 7.59-7.50(\mathrm{~m}, 2 \mathrm{H}), 7.48-7.44(\mathrm{~m}, 2 \mathrm{H}), 7.36-7.16(\mathrm{~m}, 5 \mathrm{H})$, $7.12-7.00(\mathrm{~m}, 4 \mathrm{H}) .{ }^{13} \mathrm{C} \mathrm{NMR}\left(150 \mathrm{MHz}, \mathrm{CDCl}_{3}\right)$ for the diastereoisomers $\delta 196.9,195.3,144.4,143.6$, $143.1,142.5,142.2,141.9,139.4,138.2,138.03,137.96,137.1,136.9,135.8,135.2,134.6,134.3$, $134.2,133.7,133.0,132.9,132.8,131.5,131.3,131.1,130.3,130.1,130.0,129.4,129.33,129.29$, 129.21, 129.15, 129.1, 128.9, 128.8, 128.6, 128.4, 128.3, 127.8, 127.6, 127.2, 126.6, 126.5, 126.3, 125.54, 125.49. HRMS (ESI) m/z: [M + Na $]^{+}$Calcd for $\mathrm{C}_{27} \mathrm{H}_{16} \mathrm{Cl}_{2} \mathrm{NaO}$ 449.0470; Found: 449.0467.

Note: 5ad could not be separated by silica gel column chromatography. ${ }^{1} \mathrm{H}$ NMR spectra and ${ }^{13} \mathrm{C}$ NMR pectra of the mixture were provided.

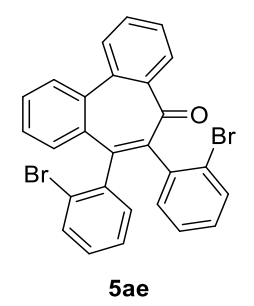

6,7-Bis(2-bromophenyl)-5H-dibenzo[a,c][7]annulen-5-one(5ae). Eluent: PE/DCM = 1:1, white solid (34.5 mg, 67\%). ${ }^{1} \mathrm{H}$ NMR (400 MHz, $\mathrm{CDCl}_{3}$ ) for the diastereoisomers $87.90-7.87(\mathrm{~m}, 1 \mathrm{H}), 7.80-$ $7.75(\mathrm{~m}, 1 \mathrm{H}), 7.72-7.68(\mathrm{~m}, 1 \mathrm{H}), 7.63-7.37(\mathrm{~m}, 5 \mathrm{H}), 7.35-7.14(\mathrm{~m}, 4 \mathrm{H}), 7.09-6.95(\mathrm{~m}, 3 \mathrm{H}) .{ }^{13} \mathrm{C}$ NMR $\left(100 \mathrm{MHz}, \mathrm{CDCl}_{3}\right.$ ) for the diastereoisomers $\delta 196.8,194.9,144.7,144.1,143.8,143.7,143.1$, 141.2, 139.7, 139.3, 138.5, 138.3, 137.3, 137.1, 137.0, 135.9, 134.7, 134.6, 133.7, 133.3, 132.7, 132.5, $132.4,131.52$, 131.46, 131.2, 131.0, 130.14, 130.05, 129.8, 129.4, 129.33, 129.30, 129.23, 129.18, 129.0, 128.7, 128.6, 128.4, 128.33, 128.25, 127.8, 127.6, 127.3, 127.0, 126.9, 126.8, 126.03, 125.96, 124.5, 124.2, 123.3, 122.7. HRMS (ESI) m/z: $[\mathrm{M}+\mathrm{Na}]^{+}$Calcd for $\mathrm{C}_{27} \mathrm{H}_{16} \mathrm{Br}_{2} \mathrm{NaO}$ 538.9440; Found: 538.9438.

Note: 5ae could not be separated by silica gel column chromatography. ${ }^{1} \mathrm{H}$ NMR spectra and ${ }^{13} \mathrm{C}$ NMR pectra of the mixture were provided.

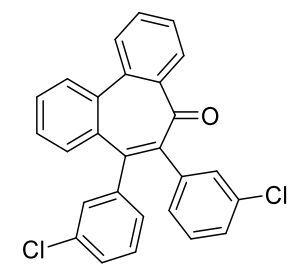

5 af

6,7-Bis(3,4-dichlorophenyl)-5H-dibenzo[a,c][7] annulen-5-one(5af). Eluent: PE/DCM = 1:1, white solid (30.6 mg, 57\%, m.p. $\left.139-140{ }^{\circ} \mathrm{C}\right) .{ }^{1} \mathrm{H}$ NMR (400 MHz, $\left.\mathrm{CDCl}_{3}\right) \delta 7.83$ (d, J= 7.8 Hz, 1H), 7.68 $(\mathrm{d}, J=7.8 \mathrm{~Hz}, 1 \mathrm{H}), 7.64-7.60(\mathrm{~m}, 1 \mathrm{H}), 7.48-7.34(\mathrm{~m}, 2 \mathrm{H}), 7.30(\mathrm{~d}, J=7.5 \mathrm{~Hz}, 1 \mathrm{H}), 7.23-7.18(\mathrm{~m}$, 
1H), $7.10-6.98(\mathrm{~m}, 5 \mathrm{H}), 6.92(\mathrm{~s}, 1 \mathrm{H}), 6.83(\mathrm{~d}, J=7.3 \mathrm{~Hz}, 1 \mathrm{H}) .{ }^{13} \mathrm{C} \mathrm{NMR}\left(150 \mathrm{MHz}, \mathrm{CDCl}_{3}\right) \delta$ 197.9, 144.2, 142.6, 141.8, 141.1, 137.8, 137.2, 136.4, 136.1, 134.2, 133.8, 131.7, 131.4, 131.0, 130.8, 130.1, 129.4, 129.2, 129.1, 128.9, 128.7, 128.5, 128.4, 127.8, 127.7, 127.6, 125.7. HRMS (ESI) m/z: [M + $\mathrm{Na}]^{+}$Calcd for $\mathrm{C}_{27} \mathrm{H}_{14} \mathrm{Cl}_{2} \mathrm{NaO}$ 449.0470; Found: 449.0472 .

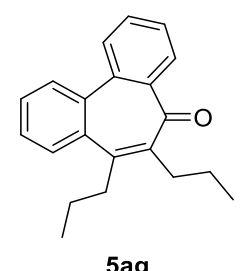

5 ag

6,7-Dipropyl-5H-dibenzo[a,c][7]annulen-5-one(5ag). Eluent: $\mathrm{PE} / \mathrm{DCM}=1: 1$, white solid (28.4 mg, 98\%, m.p. $\left.55-57^{\circ} \mathrm{C}\right) .{ }^{1} \mathrm{H}$ NMR (400 MHz, $\left.\mathrm{CDCl}_{3}\right) \delta 7.73(\mathrm{~d}, J=7.8 \mathrm{~Hz}, 1 \mathrm{H}), 7.65-7.54(\mathrm{~m}, 3 \mathrm{H})$, $7.49-7.42(\mathrm{~m}, 2 \mathrm{H}), 7.40-7.32(\mathrm{~m}, 2 \mathrm{H}), 2.73-2.48(\mathrm{~m}, 4 \mathrm{H}), 1.54-1.27(\mathrm{~m}, 4 \mathrm{H}), 0.91(\mathrm{t}, J=7.3 \mathrm{~Hz}$, $3 \mathrm{H}), 0.76(\mathrm{t}, J=7.4 \mathrm{~Hz}, 3 \mathrm{H}) .{ }^{13} \mathrm{C}$ NMR $\left(100 \mathrm{MHz}, \mathrm{CDCl}_{3}\right) \delta 201.9,145.0,144.4,140.6,137.01$, 136.96, 136.6, 131.0, 130.7, 128.2, 127.9, 127.6, 127.5, 127.1, 125.3, 34.9, 34.2, 22.9, 22.3, 14.4, 14.0. HRMS (ESI) m/z: [M + Na] $]^{+}$Calcd for $\mathrm{C}_{21} \mathrm{H}_{22} \mathrm{NaO} 313.1563$; Found: 313.1563.

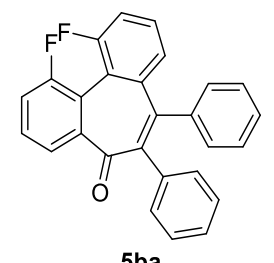

$5 \mathrm{ba}$

1,11-Difluoro-6,7-diphenyl-5H-dibenzo[a,c][7]annulen-5-one(5ba). Eluent: $\mathrm{PE} / \mathrm{DCM}=1: 1$, white solid (23.7 mg, 60\%, m.p. $\left.184-185^{\circ} \mathrm{C}\right) .{ }^{1} \mathrm{H}$ NMR $\left(400 \mathrm{MHz}, \mathrm{CDCl}_{3}\right) \delta 7.44-7.40(\mathrm{~m}, 1 \mathrm{H}), 7.35-$ $7.31(\mathrm{~m}, 1 \mathrm{H}), 7.25-7.19(\mathrm{~m}, 3 \mathrm{H}), 7.15-7.10(\mathrm{~m}, 7 \mathrm{H}), 7.05-6.95(\mathrm{~m}, 3 \mathrm{H}), 6.91(\mathrm{~d}, J=8.0 \mathrm{~Hz}, 1 \mathrm{H})$. ${ }^{13} \mathrm{C}$ NMR $\left(100 \mathrm{MHz}, \mathrm{CDCl}_{3}\right) \delta 197.9,161.4(\mathrm{~d}, J=1.7 \mathrm{~Hz}, 1 \mathrm{C}), 160.3(\mathrm{~d}, J=243.3 \mathrm{~Hz}, 1 \mathrm{C}), 159.9$ (d, $J=244.9 \mathrm{~Hz}, 1 \mathrm{C}), 158.9,147.8(\mathrm{~d}, J=1.6 \mathrm{~Hz}, 1 \mathrm{C}), 144.1,139.9(\mathrm{~d}, J=2.2 \mathrm{~Hz}, 1 \mathrm{C}), 139.8(\mathrm{~d}, J=1.8$ $\mathrm{Hz}, 1 \mathrm{C}), 140.1,134.9,131.0,130.7$ (d, $J=8.4 \mathrm{~Hz}, 1 \mathrm{C}), 130.0,129.2$ (d, $J=9.1 \mathrm{~Hz}, 1 \mathrm{C}), 128.0(\mathrm{~d}, J=$ $11.0 \mathrm{~Hz}, 1 \mathrm{C}), 127.6$ (d, $J=4.3 \mathrm{~Hz}, 1 \mathrm{C}), 126.9$ (d, $J=3.0 \mathrm{~Hz}, 1 \mathrm{C}), 120.0,119.9$ (d, $J=2.9 \mathrm{~Hz}, 1 \mathrm{C}$ ), 117.9 (d, $J=22.1 \mathrm{~Hz}, 1 \mathrm{C}), 117.1(\mathrm{~d}, J=15.7 \mathrm{~Hz}, 1 \mathrm{C}), 115.1(\mathrm{~d}, J=1.0 \mathrm{~Hz}, 1 \mathrm{C}), 114.9$ (d, $J=1.3 \mathrm{~Hz}$, 1C). ${ }^{19} \mathrm{~F}$ NMR (376 MHz, $\left.\mathrm{CDCl}_{3}\right) \delta-108.46--108.50(\mathrm{~m}),-108.60--108.64(\mathrm{~m}),-108.75--108.79$ (m), $-108.89--108.93$ (m). HRMS (ESI) m/z: $[\mathrm{M}+\mathrm{Na}]^{+} \mathrm{Calcd}$ for $\mathrm{C}_{27} \mathrm{H}_{16} \mathrm{~F}_{2} \mathrm{NaO}$ 417.1061; Found: 417.1055 .

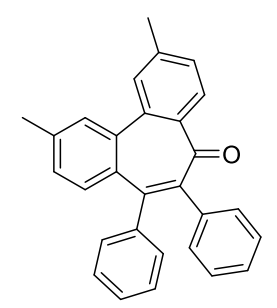

$5 \mathrm{ca}$

2,10-Dimethyl-6,7-diphenyl-5H-dibenzo[a,c][7]annulen-5-one(5ca). Eluent: $\mathrm{PE} / \mathrm{DCM}=1: 1$, red solid (37.1 mg, 96\%, m.p. $\left.166-168{ }^{\circ} \mathrm{C}\right) .{ }^{1} \mathrm{H}$ NMR $\left(600 \mathrm{MHz}, \mathrm{CDCl}_{3}\right) \delta 7.67$ (s, 1H), 7.52 (s, 1H), 7.24 $-7.22(\mathrm{~m}, 4 \mathrm{H}), 7.09-7.07$ (m, 6H), $7.01(\mathrm{dd}, J=8.2,1.3 \mathrm{~Hz}, 1 \mathrm{H}), 6.98-6.93(\mathrm{~m}, 3 \mathrm{H}), 2.51(\mathrm{~s}, 3 \mathrm{H})$, 2.41 (s, 3H). ${ }^{13} \mathrm{C}$ NMR $\left(150 \mathrm{MHz}, \mathrm{CDCl}_{3}\right) \delta 198.7,142.21,142.20,141.8,141.6,141.2,138.0,137.7$, 
136.6, 136.3, 134.6, 132.0, 131.10, 131.08, 130.4, 129.2, 129.0, 128.2, 127.8, 127.6, 127.01, 126.98, 125.9, 21.9, 21.5. HRMS (ESI) m/z: $[\mathrm{M}+\mathrm{Na}]^{+}$Calcd for $\mathrm{C}_{29} \mathrm{H}_{22} \mathrm{NaO}$ 409.1563; Found: 409.1554 .

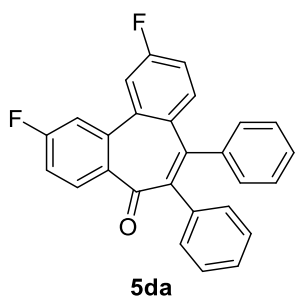

2,10-Difluoro-6,7-diphenyl-5H-dibenzo[a,c][7]annulen-5-one(5da). Eluent: $P E / D C M=1: 1$, white solid (20.0 mg, 51\%). ${ }^{1} \mathrm{H} \mathrm{NMR}\left(400 \mathrm{MHz}, \mathrm{CDCl}_{3}\right.$ ) for the regioismers $\delta 7.52-7.26(\mathrm{~m}, 3 \mathrm{H}), 7.18-$ $6.91(\mathrm{~m}, 10 \mathrm{H}), 6.91-6.73(\mathrm{~m}, 3 \mathrm{H}) .{ }^{13} \mathrm{C} \mathrm{NMR}\left(100 \mathrm{MHz}, \mathrm{CDCl}_{3}\right)$ for the regioismers $\delta 198.2,194.7$, $164.2(\mathrm{~d}, J=250.8 \mathrm{~Hz}), 161.4(\mathrm{~d}, J=249.5 \mathrm{~Hz}), 161.2(\mathrm{~d}, J=252.8 \mathrm{~Hz}), 156.4(\mathrm{~d}, J=253.0 \mathrm{~Hz})$, 144.5, 142.7 (d, $J=0.9 \mathrm{~Hz}, 1 \mathrm{H}), 141.4$ (d, $J=2.9 \mathrm{~Hz}), 140.5,140.2$ (d, $J=3.0 \mathrm{~Hz}), 138.9$ (d, $J=2.6$ $\mathrm{Hz}), 138.8(\mathrm{~d}, J=2.8 \mathrm{~Hz}), 138.7,138.4(\mathrm{~d}, J=3.2 \mathrm{~Hz}), 138.3(\mathrm{~d}, J=3.1 \mathrm{~Hz}), 134.5,134.4,134.2$, $134.0(\mathrm{~d}, J=3.3 \mathrm{~Hz}), 133.9,131.7$ (d, $J=17.3 \mathrm{~Hz}), 131.4$ (d, $J=8.5 \mathrm{~Hz}), 131.2,130.1,130.0,129.6$ (d, $J=10.3 \mathrm{~Hz}), 129.5(\mathrm{~d}, J=2.1 \mathrm{~Hz}), 128.2,128.0,127.8(\mathrm{~d}, J=9.3 \mathrm{~Hz}), 127.70(\mathrm{~d}, J=7.4 \mathrm{~Hz}), 127.68$, $127.4(\mathrm{~d}, J=42.2 \mathrm{~Hz}), 126.0(\mathrm{~d}, J=3.2 \mathrm{~Hz}), 125.8(\mathrm{~d}, J=11.0 \mathrm{~Hz}), 124.3(\mathrm{~d}, J=3.6 \mathrm{~Hz}), 116.7$ (d, $J=$ $22.7 \mathrm{~Hz}), 116.2(\mathrm{~d}, J=23.0 \mathrm{~Hz}), 115.71(\mathrm{~d}, J=40.2 \mathrm{~Hz}), 115.71(\mathrm{~d}, J=2.7 \mathrm{~Hz}), 115.4(\mathrm{~d}, J=4.0 \mathrm{~Hz})$, $115.1(\mathrm{~d}, J=5.7 \mathrm{~Hz}) .{ }^{19} \mathrm{~F}$ NMR $\left(376 \mathrm{MHz}, \mathrm{CDCl}_{3}\right) \delta-101.40--101.44(\mathrm{~m}),-108.27--108.33(\mathrm{~m})$, $-112.44--112.50(\mathrm{~m}),-119.17--119.20(\mathrm{~m})$. (The fluorine spectrum shows that the ratio of products is 1:1.1). HRMS (ESI) m/z: [M + Na] $]^{+}$Calcd for $\mathrm{C}_{27} \mathrm{H}_{16} \mathrm{~F}_{2} \mathrm{NaO}$ 417.1061; Found: 417.1054.

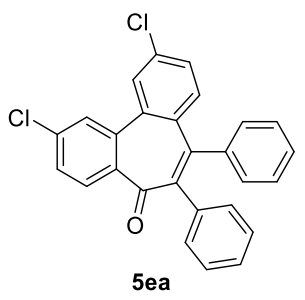

2,10-Dichloro-6,7-diphenyl-5H-dibenzo[a,c][7]annulen-5-one(5ea). Eluent: $\mathrm{PE} / \mathrm{DCM}=1: 1$, colorless liquid (42.3 mg, 99\%). ${ }^{1} \mathrm{H}$ NMR (400 MHz, $\left.\mathrm{CDCl}_{3}\right) \delta 7.85(\mathrm{~d}, J=1.8 \mathrm{~Hz}, 1 \mathrm{H}), 7.69(\mathrm{~d}, J=$ $2.1 \mathrm{~Hz}, 1 \mathrm{H}), 7.44$ (dd, $J=8.2,1.8 \mathrm{~Hz}, 1 \mathrm{H}), 7.29$ (d, $J=8.2 \mathrm{~Hz}, 1 \mathrm{H}), 7.26-7.18$ (m, 3H), $7.17-7.09$ $(\mathrm{m}, 6 \mathrm{H}), 7.06(\mathrm{~d}, J=8.6 \mathrm{~Hz}, 1 \mathrm{H}), 6.95-6.93(\mathrm{~m}, 2 \mathrm{H}) .{ }^{13} \mathrm{C} \mathrm{NMR}\left(150 \mathrm{MHz}, \mathrm{CDCl}_{3}\right) \delta 197.3,143.0$, $142.8,140.9,140.7,137.8,137.3,136.7,135.9,135.4,134.0,133.7,131.0,130.2$, 130.1, 129.0, 128.5, 128.1, 128.0, 127.8, 127.6, 127.5, 127.4. HRMS (ESI) m/z: $[\mathrm{M}+\mathrm{Na}]^{+}$Calcd for $\mathrm{C}_{27} \mathrm{H}_{16} \mathrm{Cl}_{2} \mathrm{NaO}$ 449.0470; Found:449.0460.

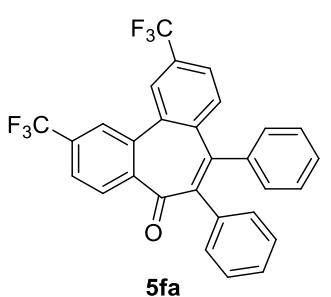

6,7-Diphenyl-2,10-bis(trifluoromethyl)-5H-dibenzo[a,c][7]annulen-5-one(5fa).Eluent: PE/DCM = 1:1, white solid (48.9 mg, 99\%, m.p. $\left.162-163{ }^{\circ} \mathrm{C}\right) .{ }^{1} \mathrm{H}$ NMR $\left(400 \mathrm{MHz}, \mathrm{CDCl}_{3}\right) \delta 8.24(\mathrm{~s}, 1 \mathrm{H}), 8.06(\mathrm{~s}$, 
1H), $7.83(\mathrm{~d}, J=7.9 \mathrm{~Hz}, 1 \mathrm{H}), 7.59(\mathrm{~d}, J=8.1 \mathrm{~Hz}, 1 \mathrm{H}), 7.53(\mathrm{~d}, J=7.9 \mathrm{~Hz}, 1 \mathrm{H}), 7.40-7.31(\mathrm{~m}, 3 \mathrm{H})$, $7.24-7.21(\mathrm{~m}, 6 \mathrm{H}), 7.05-7.04(\mathrm{~m}, 2 \mathrm{H}) .{ }^{13} \mathrm{C}$ NMR $\left(100 \mathrm{MHz}, \mathrm{CDCl}_{3}\right) \delta$ 197.4, 147.0, 144.2, 140.7, $140.1,136.8,135.7,134.7,133.2$ (q, $J=32.9 \mathrm{~Hz}, 1 \mathrm{C}), 132.9,131.0,130.1,130.3$ (q, $J=32.9 \mathrm{~Hz}, 1 \mathrm{C}$ ), 128.3, 128.0, 127.9, 127.4 (q, $J=3.9 \mathrm{~Hz}, 1 \mathrm{C}), 126.4,125.7(\mathrm{q}, J=3.5 \mathrm{~Hz}, 1 \mathrm{C}), 125.6(\mathrm{q}, J=3.7 \mathrm{~Hz}$, 1C), 124.6 (q, $J=3.5 \mathrm{~Hz}, 1 \mathrm{C}), 123.8(\mathrm{q}, J=272.9 \mathrm{~Hz}, 1 \mathrm{C}) .{ }^{19} \mathrm{~F}$ NMR $\left(376 \mathrm{MHz}, \mathrm{CDCl}_{3}\right) \delta-62.55$, -62.63. HRMS (ESI) m/z: [M + Na $]^{+}$Calcd for $\mathrm{C}_{29} \mathrm{H}_{16} \mathrm{~F}_{6} \mathrm{NaO}$ 517.0998; Found: 517.1000.

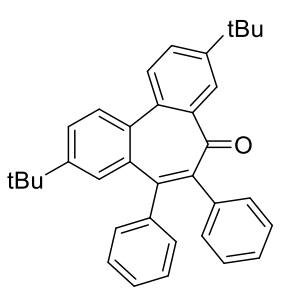

$5 \mathrm{ga}$

3,9-Di-tert-butyl-6,7-diphenyl-5H-dibenzo[a,c][7]annulen-5-one(5ga). Eluent: $\mathrm{PE} / \mathrm{DCM}=1: 1$, white solid (46.5 mg, 99\%, m.p. $\left.200-201{ }^{\circ} \mathrm{C}\right) .{ }^{1} \mathrm{H}$ NMR $\left(400 \mathrm{MHz}, \mathrm{CDCl}_{3}\right) \delta 7.85(\mathrm{~d}, J=8.3 \mathrm{~Hz}, 1 \mathrm{H})$, $7.73-7.66(\mathrm{~m}, 2 \mathrm{H}), 7.43(\mathrm{dd}, J=8.3,2.0 \mathrm{~Hz}, 1 \mathrm{H}), 7.39(\mathrm{~d}, J=2.0 \mathrm{~Hz}, 1 \mathrm{H}), 7.33(\mathrm{~d}, J=3.6 \mathrm{~Hz}, 1 \mathrm{H})$, $7.31(\mathrm{~d}, J=2.4 \mathrm{~Hz}, 1 \mathrm{H}), 7.17-7.08(\mathrm{~m}, 7 \mathrm{H}), 7.00(\mathrm{~d}, J=3.6 \mathrm{~Hz}, 1 \mathrm{H}), 6.98(\mathrm{~d}, J=2.3 \mathrm{~Hz}, 1 \mathrm{H}), 1.35(\mathrm{~s}$, 9H), 1.16 (s, 9H). ${ }^{13} \mathrm{C}$ NMR $\left(150 \mathrm{MHz}, \mathrm{CDCl}_{3}\right) \delta 199.3,151.1,149.6,144.0,142.8,142.2,141.7,136.7$, 136.5, 134.9, 133.9, 131.1, 130.6, 130.2, 129.1, 128.5, 128.4, 127.7, 127.6, 126.98, 126.96, 125.3, 122.5, 35.0, 34.6, 31.4, 31.1. HRMS (ESI) m/z: $[\mathrm{M}+\mathrm{Na}]^{+}$Calcd for $\mathrm{C}_{35} \mathrm{H}_{34} \mathrm{NaO}$ 493.2502; Found: 493.2495 .

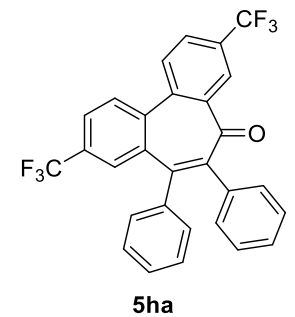

6,7-Diphenyl-3,9-bis(trifluoromethyl)-5H-dibenzo[a,c][7]annulen-5-one(5ha). Eluent: PE/DCM = 1:1, colorless liquid (48.9 mg, 99\%). ${ }^{1} \mathrm{H}$ NMR $\left(400 \mathrm{MHz}, \mathrm{CDCl}_{3}\right) \delta 8.03(\mathrm{~d}, J=8.3 \mathrm{~Hz}, 1 \mathrm{H}), 7.92(\mathrm{~d}, J$ $=8.3 \mathrm{~Hz}, 1 \mathrm{H}), 7.85(\mathrm{~d}, J=8.3 \mathrm{~Hz}, 1 \mathrm{H}), 7.71-7.60(\mathrm{~m}, 2 \mathrm{H}), 7.41(\mathrm{~s}, 1 \mathrm{H}), 7.28-7.23(\mathrm{~m}, 2 \mathrm{H}), 7.21-$ $7.07(\mathrm{~m}, 6 \mathrm{H}), 6.96-6.94(\mathrm{~m}, 2 \mathrm{H}) .{ }^{13} \mathrm{C}$ NMR $\left(150 \mathrm{MHz}, \mathrm{CDCl}_{3}\right) \delta 196.9,144.8,144.0,141.0,139.9$, 139.3, 138.30, 138.26, 134.9, 131.5, 131.3 (q, $J=33.8 \mathrm{~Hz}, 1 \mathrm{C}), 130.9,130.4$ (q, $J=32.9 \mathrm{~Hz}, 1 \mathrm{C})$, 130.2, 129.6, 129.0 (q, $J=3.6 \mathrm{~Hz}, 1 \mathrm{C}), 128.4,128.0,127.9,127.7$ (q, $J=3.3 \mathrm{~Hz}, 1 \mathrm{C}), 124.7$ (q, $J=3.4$ $\mathrm{Hz}, 1 \mathrm{C}), 123.7$ (q, $J=272.6 \mathrm{~Hz}, 1 \mathrm{C}), 123.6$ (q, $J=272.4 \mathrm{~Hz}, 1 \mathrm{C}), 123.0(\mathrm{q}, J=3.9 \mathrm{~Hz}, 1 \mathrm{C}) .{ }^{19} \mathrm{~F}$ NMR $\left(376 \mathrm{MHz}, \mathrm{CDCl}_{3}\right) \delta-62.59$, -62.93. HRMS (ESI) $\mathrm{m} / \mathrm{z}$ : $[\mathrm{M}+\mathrm{Na}]^{+} \mathrm{Calcd}$ for $\mathrm{C}_{29} \mathrm{H}_{16} \mathrm{~F}_{6} \mathrm{NaO}$ 517.0998; Found: 517.0997.

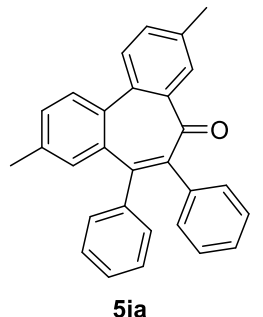


3,9-Dimethyl-6,7-diphenyl-5H-dibenzo[a,c][7]annulen-5-one(5ia). Eluent: $\mathrm{PE} / \mathrm{DCM}=1: 1$, yellow liquid $(37.0 \mathrm{mg}, 96 \%) .{ }^{1} \mathrm{H}$ NMR $\left(600 \mathrm{MHz}, \mathrm{CDCl}_{3}\right) \delta 7.78(\mathrm{~d}, J=8.1 \mathrm{~Hz}, 1 \mathrm{H}), 7.63(\mathrm{~d}, J=8.1 \mathrm{~Hz}, 1 \mathrm{H})$, $7.46(\mathrm{~d}, J=8.0 \mathrm{~Hz}, 1 \mathrm{H}), 7.28-7.27(\mathrm{~m}, 2 \mathrm{H}), 7.22(\mathrm{~d}, J=8.0 \mathrm{~Hz}, 1 \mathrm{H}), 7.17(\mathrm{~s}, 1 \mathrm{H}), 7.14-7.11(\mathrm{~m}$, $6 \mathrm{H}), 7.00-6.99(\mathrm{~m}, 2 \mathrm{H}), 6.92(\mathrm{~s}, 1 \mathrm{H}), 2.39(\mathrm{~s}, 3 \mathrm{H}), 2.24(\mathrm{~s}, 3 \mathrm{H}) .{ }^{13} \mathrm{C} \mathrm{NMR}\left(150 \mathrm{MHz}, \mathrm{CDCl}_{3}\right) \delta 198.8$, 159.2, 157.8, 144.8, 142.19, 142.17, 141.3, 137.9, 136.3, 131.7, 131.0, 130.7, 130.4, 130.0, 129.5, 127.9, 127.6, 127.2, 127.1, 118.7, 116.5, 114.6, 108.8, 55.7, 55.3. HRMS (ESI) m/z: $[\mathrm{M}+\mathrm{Na}]^{+} \mathrm{Calcd}$ for $\mathrm{C}_{29} \mathrm{H}_{22} \mathrm{NaO}$ 409.1563; Found: 409.1563 .

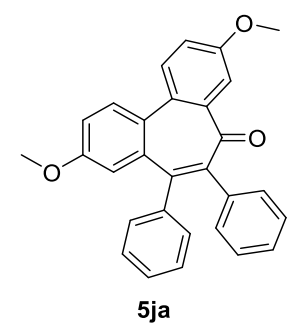

3,9-Dimethoxy-6,7-diphenyl-5H-dibenzo[a,c][7]annulen-5-one(5ja). Eluent: $\mathrm{PE} / \mathrm{DCM}=1: 1$, pale yellow liquid (35.8 mg, 86\%). ${ }^{1} \mathrm{H}$ NMR $\left(600 \mathrm{MHz}, \mathrm{CDCl}_{3}\right) \delta 7.75(\mathrm{~d}, J=8.7 \mathrm{~Hz}, 1 \mathrm{H}), 7.61(\mathrm{~d}, J=8.8$ $\mathrm{Hz}, 1 \mathrm{H}), 7.27-7.24(\mathrm{~m}, 2 \mathrm{H}), 7.18(\mathrm{dd}, J=8.7,2.8 \mathrm{~Hz}, 1 \mathrm{H}), 7.12-7.10(\mathrm{~m}, 6 \mathrm{H}), 6.99-6.95(\mathrm{~m}, 3 \mathrm{H})$, $6.84(\mathrm{~d}, J=2.8 \mathrm{~Hz}, 1 \mathrm{H}), 6.59(\mathrm{~d}, J=2.7 \mathrm{~Hz}, 1 \mathrm{H}), 3.80(\mathrm{~s}, 3 \mathrm{H}), 3.62(\mathrm{~s}, 3 \mathrm{H}) .{ }^{13} \mathrm{C}$ NMR $(150 \mathrm{MHz}$, $\left.\mathrm{CDCl}_{3}\right) \delta 198.8,159.2,157.8,144.8,142.19,142.17,141.3,137.9,136.3,131.7,131.0,130.7,130.4$, 130.0, 129.5, 127.9, 127.6, 127.2, 127.1, 118.7, 116.5, 114.6, 108.8, 55.7, 55.3. HRMS (ESI) m/z: [M $+\mathrm{Na}]^{+}$Calcd for $\mathrm{C}_{29} \mathrm{H}_{22} \mathrm{NaO}_{3} 441.1461$; Found: 441.1459 .<smiles>O=c1c(-c2ccccc2)c(-c2ccccc2)c2cc(Cl)ccc2c2cc(Cl)ccc12</smiles>

3,9-Dichloro-6,7-diphenyl-5H-dibenzo[a,c][7]annulen-5-one(5ka). Eluent: PE/DCM = 1:1, colorless liquid (42.3 mg, 99\%). ${ }^{1} \mathrm{H}$ NMR (600 MHz, $\left.\mathrm{CDCl}_{3}\right) \delta 7.79(\mathrm{~d}, J=8.5 \mathrm{~Hz}, 1 \mathrm{H}), 7.66-7.60(\mathrm{~m}, 2 \mathrm{H})$, $7.38(\mathrm{dd}, J=8.5,2.2 \mathrm{~Hz}, 1 \mathrm{H}), 7.34(\mathrm{~d}, J=2.2 \mathrm{~Hz}, 1 \mathrm{H}), 7.25-7.24(\mathrm{~m}, 2 \mathrm{H}), 7.19-7.09$ (m, 7H), 7.00 $-6.94(\mathrm{~m}, 2 \mathrm{H}) .{ }^{13} \mathrm{C}$ NMR $\left(150 \mathrm{MHz}, \mathrm{CDCl}_{3}\right) \delta 197.1,145.2,143.4,140.9,140.2,138.7,135.2,135.03$, 134.97, 133.9, 133.7, 131.9, 131.6, 131.2, 131.0, 130.2, 130.0, 128.5, 128.2, 127.9, 127.7, 127.6, 125.4. HRMS (ESI) m/z: [M + Na $]^{+}$Calcd for $\mathrm{C}_{27} \mathrm{H}_{21} \mathrm{Cl}_{2} \mathrm{NaO}$ 449.0470; Found: 449.0467.<smiles></smiles>

10-(Trifluoromethyl)-9,10-dihydrophenanthren-9-yl 4-methylbenzoate(6). Eluent: PE/EA = 30:1, colorless liquid (14.1 mg, 37\%). ${ }^{1} \mathrm{H}$ NMR $\left(600 \mathrm{MHz}, \mathrm{CDCl}_{3}\right) \delta 7.94(\mathrm{~d}, J=7.8 \mathrm{~Hz}, 1 \mathrm{H}), 7.89(\mathrm{~d}, J=$ $7.8 \mathrm{~Hz}, 1 \mathrm{H}), 7.65(\mathrm{~d}, J=8.2 \mathrm{~Hz}, 2 \mathrm{H}), 7.61(\mathrm{~d}, J=7.5 \mathrm{~Hz}, 1 \mathrm{H}), 7.53-7.47(\mathrm{~m}, 2 \mathrm{H}), 7.41-7.34(\mathrm{~m}$, $3 \mathrm{H}), 7.09(\mathrm{~d}, J=8.2 \mathrm{~Hz}, 2 \mathrm{H}), 6.52(\mathrm{~d}, J=1.2 \mathrm{~Hz}, 1 \mathrm{H}), 3.99(\mathrm{q}, J=9.1 \mathrm{~Hz}, 1 \mathrm{H}), 2.32(\mathrm{~s}, 3 \mathrm{H}) .{ }^{13} \mathrm{C}$ NMR 
$\left(150 \mathrm{MHz}, \mathrm{CDCl}_{3}\right) \delta 165.7,144.1,134.2,134.1,131.7,131.0,130.6,130.4,129.9,129.7,129.1,128.5$, 128.3, 127.0, 126.9 (q, $J=281.3 \mathrm{~Hz}, 1 \mathrm{C}), 126.4,124.3,124.0,68.3(\mathrm{q}, J=1.9 \mathrm{~Hz}, 1 \mathrm{C}), 48.1$ (q, $J=$ $26.6 \mathrm{~Hz}, 1 \mathrm{C}), 21.8 .{ }^{19} \mathrm{~F}$ NMR $\left(565 \mathrm{MHz}, \mathrm{CDCl}_{3}\right) \delta-68.58$ (d). HRMS (ESI) $\mathrm{m} / \mathrm{z}:[\mathrm{M}+\mathrm{Na}]^{+} \mathrm{Calcd}$ for $\mathrm{C}_{16} \mathrm{H}_{10} \mathrm{~F}_{3} \mathrm{NaO}_{2}$ 405.1073; Found: 405.1073 .

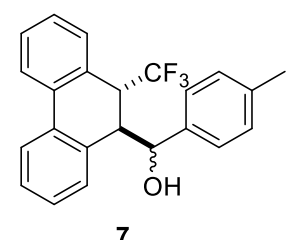

p-Tolyl(10-(trifluoromethyl)-9,10-dihydrophenanthren-9-yl)methanol(7). Eluent: PE, colorless solid (17.7 mg, 48\%, m.p. $\left.117-118^{\circ} \mathrm{C}\right) .{ }^{1} \mathrm{H}$ NMR $\left(400 \mathrm{MHz}, \mathrm{CDCl}_{3}\right) \delta 7.88(\mathrm{~d}, J=7.7 \mathrm{~Hz}, 1 \mathrm{H}), 7.75$ (d, $J=7.7 \mathrm{~Hz}, 1 \mathrm{H}), 7.50-7.44(\mathrm{~m}, 2 \mathrm{H}), 7.38-7.34(\mathrm{~m}, 1 \mathrm{H}), 7.26-7.21(\mathrm{~m}, 1 \mathrm{H}), 7.02(\mathrm{~d}, J=7.9 \mathrm{~Hz}$, 2H), $6.93-6.88(\mathrm{~m}, 3 \mathrm{H}), 6.36(\mathrm{~d}, J=7.2 \mathrm{~Hz}, 1 \mathrm{H}), 4.32-4.23(\mathrm{~m}, 2 \mathrm{H}), 3.41(\mathrm{~d}, J=9.4 \mathrm{~Hz}, 1 \mathrm{H}), 2.31$ $(\mathrm{s}, 3 \mathrm{H}), 1.88(\mathrm{~s}, 1 \mathrm{H}) .{ }^{13} \mathrm{C} \mathrm{NMR}\left(100 \mathrm{MHz}, \mathrm{CDCl}_{3}\right) \delta 139.2,137.9,135.0,133.3,133.0,132.0,130.6$, $129.5,128.9,128.1,127.9,127.5,126.90$ (q, $J=281.4 \mathrm{~Hz}, 1 \mathrm{C}), 126.86,123.9,123.5,46.7,43.9$ (q, $J=$ $26.7 \mathrm{~Hz}, 1 \mathrm{C}$ ), 21.3. ${ }^{19} \mathrm{~F}$ NMR (376 MHz, $\mathrm{CDCl}_{3}$ ) $\delta-69.35$ (d). HRMS (ESI) m/z: $[\mathrm{M}+\mathrm{Na}]^{+} \mathrm{Calcd}$ for $\mathrm{C}_{23} \mathrm{H}_{19} \mathrm{~F}_{3} \mathrm{NaO}$ 391.1280; Found: 391.1281 .<smiles>Cc1ccc(C(O)C2c3ccccc3-c3ccccc3[C@@H]2F)cc1</smiles>

p-Tolyl(10-(trifluoromethyl)-9,10-dihydrophenanthren-9-yl)methanol(7'). Eluent: PE，colorless solid (16.6 mg, 45\%, m.p. $\left.115-116^{\circ} \mathrm{C}\right) .{ }^{1} \mathrm{H}$ NMR $\left(400 \mathrm{MHz}, \mathrm{CDCl}_{3}\right) \delta 7.86-7.82(\mathrm{~m}, 2 \mathrm{H}), 7.51-$ 7.39 (m, 3H), $7.38-7.29$ (m, 2H), $7.18(\mathrm{~d}, J=7.8 \mathrm{~Hz}, 2 \mathrm{H}), 7.11-7.07$ (m, 3H), 4.20 (d, J = 9.5 Hz, $1 \mathrm{H}), 3.37$ (d, $J=9.5 \mathrm{~Hz}, 1 \mathrm{H}), 3.10(\mathrm{q}, J=9.7 \mathrm{~Hz}, 1 \mathrm{H}), 2.38(\mathrm{~s}, 3 \mathrm{H}), 1.91(\mathrm{~s}, 1 \mathrm{H}) .{ }^{13} \mathrm{C}$ NMR $(100 \mathrm{MHz}$, $\left.\mathrm{CDCl}_{3}\right) \delta 138.5,138.0,134.9,133.9,133.1,131.5,131.0,129.63,129.60,128.6,128.2,127.4,126.8$, 126.3 (q, $J=281.5 \mathrm{~Hz}, 1 \mathrm{C}), 124.24,124.21,75.2,46.6,44.9$ (q, $J=27.0 \mathrm{~Hz}, 1 \mathrm{C}), 21.4 .{ }^{19} \mathrm{~F}$ NMR $(376$ $\left.\mathrm{MHz}, \mathrm{CDCl}_{3}\right) \delta-69.77$ (d). HRMS (ESI) m/z: $[\mathrm{M}+\mathrm{Na}]^{+}$Calcd for $\mathrm{C}_{23} \mathrm{H}_{19} \mathrm{~F}_{3} \mathrm{NaO}$ 391.1280; Found: 391.1277 .<smiles>FC(F)(F)c1c(CCl)c2ccccc2c2ccccc12</smiles>

8

9-(4-Methylbenzyl)-10-(trifluoromethyl)phenanthrene(8). Eluent: PE, white solid (29.3 mg, 84\%, m.p. $\left.106-107^{\circ} \mathrm{C}\right) .{ }^{1} \mathrm{H}$ NMR $\left(600 \mathrm{MHz}, \mathrm{CDCl}_{3}\right) \delta 8.75(\mathrm{~d}, J=8.0 \mathrm{~Hz}, 1 \mathrm{H}), 8.73(\mathrm{~d}, J=8.3 \mathrm{~Hz}, 1 \mathrm{H})$, $8.36(\mathrm{~d}, J=8.2 \mathrm{~Hz}, 1 \mathrm{H}), 8.10(\mathrm{~d}, J=8.4 \mathrm{~Hz}, 1 \mathrm{H}), 7.74-7.66(\mathrm{~m}, 3 \mathrm{H}), 7.55-7.52(\mathrm{~m}, 1 \mathrm{H}), 7.08(\mathrm{~d}, J$ $=8.0 \mathrm{~Hz}, 2 \mathrm{H}), 7.03(\mathrm{~d}, J=8.0 \mathrm{~Hz}, 2 \mathrm{H}), 4.75(\mathrm{~s}, 2 \mathrm{H}), 2.31(\mathrm{~s}, 3 \mathrm{H}) \cdot{ }^{13} \mathrm{C} \mathrm{NMR}\left(150 \mathrm{MHz}, \mathrm{CDCl}_{3}\right) \delta$ 137.0, 136.2, 135.6, 131.9, 131.0, 130.6, 129.4, 128.6, 127.89, 127.86, 127.6, 127.3, 127.1, 127.0, $126.03(\mathrm{q}, J=5.2 \mathrm{~Hz}, 1 \mathrm{C}), 125.97(\mathrm{q}, J=277.8 \mathrm{~Hz}, 1 \mathrm{C}), 124.5(\mathrm{q}, J=27.6 \mathrm{~Hz}, 1 \mathrm{C}), 123.03,123.00$, 
$35.7(\mathrm{q}, J=4.8 \mathrm{~Hz}, 1 \mathrm{C}), 21.1 .{ }^{19} \mathrm{~F} \mathrm{NMR}\left(56 \mathrm{C} 5 \mathrm{MHz}, \mathrm{CDCl}_{3}\right) \delta-50.56 . \mathrm{HRMS}(\mathrm{ESI}) \mathrm{m} / \mathrm{z}:[\mathrm{M}+\mathrm{Na}]^{+}$ Calcd for $\mathrm{C}_{16} \mathrm{H}_{10} \mathrm{~F}_{3} \mathrm{Na}$ 373.1175; Found: 373.1177 .<smiles>C=C(Cl)C1c2ccccc2-c2ccccc2C1C(F)(F)F</smiles>

9

9-(1-(p-Tolyl)vinyl)-10-(trifluoromethyl)-9,10-dihydrophenanthrene(9). Eluent: PE, colorless liquid (29.2 mg, 80\%). ${ }^{1} \mathrm{H}$ NMR (400 MHz, $\left.\mathrm{CDCl}_{3}\right) \delta 7.92-7.90$ (m, 2H), $7.50-7.48(\mathrm{~m}, 2 \mathrm{H}), 7.41-7.37$ (m, 1H), $7.36-7.31(\mathrm{~m}, 2 \mathrm{H}), 7.31-7.28(\mathrm{~m}, 2 \mathrm{H}), 7.28-7.25(\mathrm{~m}, 2 \mathrm{H}), 7.12(\mathrm{~d}, J=7.5 \mathrm{~Hz}, 1 \mathrm{H}), 5.14$ (s, 1H), 4.59 (s, 1H), $4.39-4.34(\mathrm{~m}, 1 \mathrm{H}), 3.53$ (q, $J=9.8 \mathrm{~Hz}, 1 \mathrm{H}), 2.45$ (s, 3H). ${ }^{13} \mathrm{C}$ NMR (150 MHz, $\left.\mathrm{CDCl}_{3}\right) \delta 148.1,137.9,137.3,135.0,134.9,134.1,131.8,129.9,129.6,129.4,128.5,128.2,127.9$, 127.1, 126.7, 126.6 (q, $J=282.2 \mathrm{~Hz}, 1 \mathrm{C}), 124.0,123.8,116.8,46.7$ (q, $J=26.2 \mathrm{~Hz}, 1 \mathrm{C}), 42.6,21.3 .{ }^{19} \mathrm{~F}$ NMR (376 MHz, $\left.\mathrm{CDCl}_{3}\right) \delta-69.96$ (d). HRMS (ESI) m/z: [M + Na] $]^{+}$Calcd for $\mathrm{C}_{24} \mathrm{H}_{19} \mathrm{~F}_{3} \mathrm{Na} 387.1331$; Found: 387.1331.<smiles>OC1C(c2ccccc2)=C(c2ccccc2)c2ccccc2-c2ccccc21</smiles>

10

6,7-diphenyl-5H-dibenzo[a,c][7]annulen-5-ol(10). Eluent: PE, colorless liquid (35.5 mg, 99\%). ${ }^{1} \mathrm{H}$ NMR $\left(400 \mathrm{MHz}, \mathrm{CDCl}_{3}\right)$ for major product $\delta 7.77(\mathrm{dd}, J=7.9,1.1 \mathrm{~Hz}, 1 \mathrm{H}), 7.74-7.68(\mathrm{~m}, 1 \mathrm{H}), 7.45$ - 7.41 (m, 3H), $7.20-7.16(\mathrm{~m}, 4 \mathrm{H}), 7.09-7.07$ (m, 2H), $7.03-6.99(\mathrm{~m}, 3 \mathrm{H}), 6.96-6.92(\mathrm{~m}, 2 \mathrm{H}), 6.86-$ $6.79(\mathrm{~m}, 2 \mathrm{H}), 5.09(\mathrm{~d}, J=6.9 \mathrm{~Hz}, 1 \mathrm{H}), 2.14(\mathrm{~d}, J=7.4 \mathrm{~Hz}, 1 \mathrm{H}) .{ }^{13} \mathrm{C} \mathrm{NMR}\left(151 \mathrm{MHz}, \mathrm{CDCl}_{3}\right)$ for mixtures $\delta 145.6,144.6,144.0,142.9,142.8,141.7,141.5,139.7,139.0,138.8,138.2,136.0,135.9$, 135.7, 135.2, 134.3, 131.6, 131.5, 131.4, 131.1, 130.7, 130.3, 130.0, 129.6, 129.0, 128.9, 128.00, 127.95, 127.9, 127.8, 127.6, 127.42, 127.38, 127.2, 127.0, 126.8, 126.60, 126.58, 126.4, 120.4, 79.0, 70.6. HRMS (ESI) m/z: $[\mathrm{M}+\mathrm{Na}]^{+} \mathrm{Calcd}$ for $\mathrm{C}_{27} \mathrm{H}_{20} \mathrm{NaO}$ 383.1406; Found: 383.1406 .

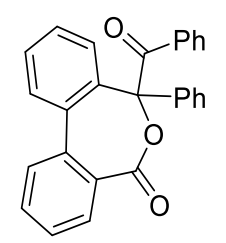

11

7-benzoyl-7-phenyldibenzo[c,e]oxepin-5(7H)-one(11). Eluent: PE/EA $=1: 30$, white solid $(20.1 \mathrm{mg}$, $54 \%$, m.p. $\left.210-211^{\circ} \mathrm{C}\right) .{ }^{1} \mathrm{H}$ NMR $\left(600 \mathrm{MHz}, \mathrm{CDCl}_{3}\right) \delta 8.37(\mathrm{~d}, J=8.0 \mathrm{~Hz}, 1 \mathrm{H}), 7.71-7.68(\mathrm{~m}, 1 \mathrm{H})$, $7.63-7.61(\mathrm{~m}, 1 \mathrm{H}), 7.59$ (d, $J=7.6 \mathrm{~Hz}, 1 \mathrm{H}), 7.55(\mathrm{~d}, J=7.7 \mathrm{~Hz}, 1 \mathrm{H}), 7.51-7.46(\mathrm{~m}, 2 \mathrm{H}), 7.40-7.33$ (m, 4H), $7.28(\mathrm{~d}, J=7.8 \mathrm{~Hz}, 1 \mathrm{H}), 7.19-7.15(\mathrm{~m}, 4 \mathrm{H}), 7.11(\mathrm{~d}, J=7.8 \mathrm{~Hz}, 1 \mathrm{H}), 6.66(\mathrm{~d}, J=7.9 \mathrm{~Hz}$, 1H). ${ }^{13} \mathrm{C}$ NMR $\left(150 \mathrm{MHz}, \mathrm{CDCl}_{3}\right) \delta 197.1,168.0,141.8,140.2,137.8,135.5,134.9,133.6,133.0$, 131.9, 130.0, 129.94, 129.87, 129.5, 129.2, 129.0, 128.9, 128.6, 128.4, 128.2, 128.1, 127.5, 127.1, 91.8. HRMS (ESI) m/z: [M + Na] $]^{+}$Calcd for $\mathrm{C}_{27} \mathrm{H}_{18} \mathrm{NaO}_{3} 413.1148$; Found: 413.1146 . 


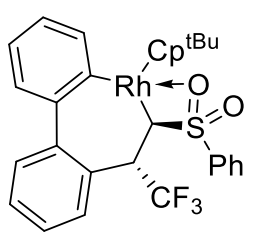

3-I

Product 3-I. Eluent: PE/EA = 1:10, red solid (6.1 mg, 40\%, m.p. $\left.190-191{ }^{\circ} \mathrm{C}\right) .{ }^{1} \mathrm{H}$ NMR (600 MHz, $\left.\mathrm{CDCl}_{3}\right) \delta 7.83(\mathrm{~d}, J=7.6 \mathrm{~Hz}, 2 \mathrm{H}), 7.78(\mathrm{dd}, J=7.4,1.3 \mathrm{~Hz}, 1 \mathrm{H}), 7.63-7.60(\mathrm{~m}, 1 \mathrm{H}), 7.53-7.51(\mathrm{~m}$, $2 \mathrm{H}), 7.38(\mathrm{~d}, J=7.9 \mathrm{~Hz}, 1 \mathrm{H}), 7.31(\mathrm{dd}, J=7.5,1.3 \mathrm{~Hz}, 1 \mathrm{H}), 7.28-7.26(\mathrm{~m}, 2 \mathrm{H}), 7.18-7.12(\mathrm{~m}, 3 \mathrm{H})$, $5.60(\mathrm{dd}, J=10.4,4.5 \mathrm{~Hz}, 1 \mathrm{H}), 5.44(\mathrm{~d}, J=2.1 \mathrm{~Hz}, 1 \mathrm{H}), 4.16-4.09(\mathrm{~m}, 1 \mathrm{H}), 2.06(\mathrm{~d}, J=1.9 \mathrm{~Hz}, 1 \mathrm{H})$, $1.93(\mathrm{~d}, J=14.6 \mathrm{~Hz}, 1 \mathrm{H}), 1.55$ (s, 9H), 1.37 (s, 9H), 1.15 (d, $J=14.6 \mathrm{~Hz}, 1 \mathrm{H}), 0.95$ (s, 9H), 0.69 (s, 9H). ${ }^{13} \mathrm{C}$ NMR $\left(150 \mathrm{MHz}, \mathrm{CDCl}_{3}\right) \delta 147.7,146.7,144.6,137.3,133.4,133.2,130.3,129.2,128.6$, 126.81, 126.75, 125.5, 125.4, 123.8, 108.29, 108.26, 107.0, 106.9, 90.5, 56.5, 56.4, 45.7, 45.5, 42.0, 39.7, 39.6, 38.4, 33.7, 31.9, 31.8, 31.5, 30.8, 30.5. ${ }^{19} \mathrm{~F}$ NMR (565 MHz, $\left.\mathrm{CDCl}_{3}\right) \delta-63.72$ (d). HRMS (ESI) $\mathrm{m} / \mathrm{z}:[\mathrm{M}+\mathrm{Na}]^{+}$Calcd for $\mathrm{C}_{39} \mathrm{H}_{46} \mathrm{~F}_{3} \mathrm{O}_{2} \mathrm{RhSNa}$ 761.2118; Found: 761.2127. 


\section{NMR Spectrum and Mass Spectrogram}

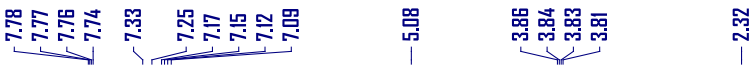

${ }^{1} \mathrm{H} \mathrm{NMR}\left(600 \mathrm{MHz}, \mathrm{CDCl}_{3}\right)$

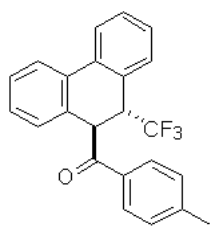

3aa

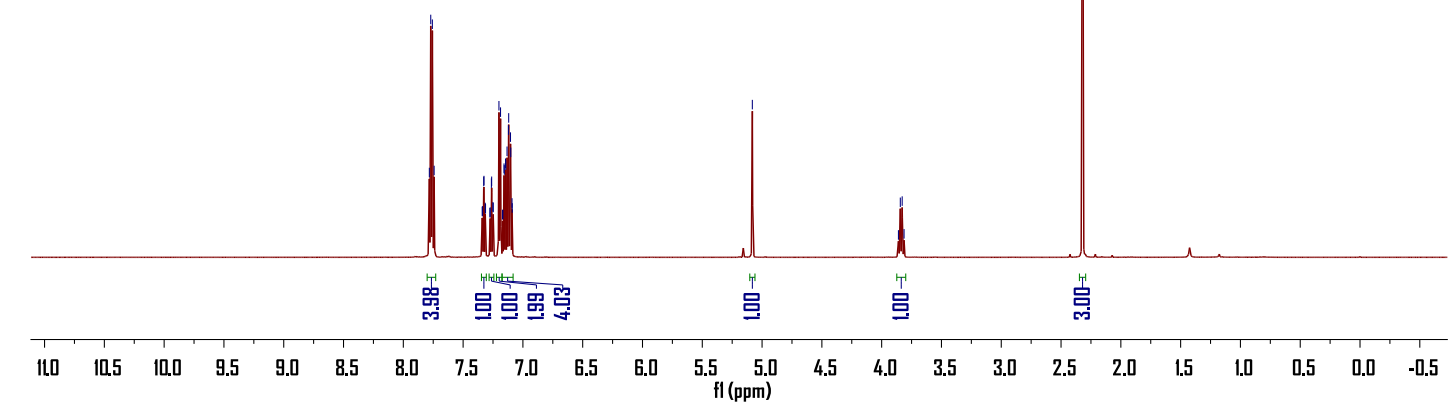

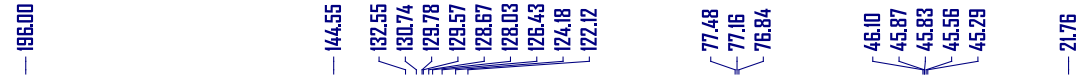

${ }^{13} \mathrm{C} \mathrm{NMR}\left(100 \mathrm{MHz}, \mathrm{CDCl}_{3}\right)$

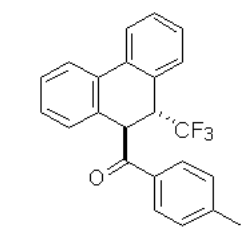

3aa

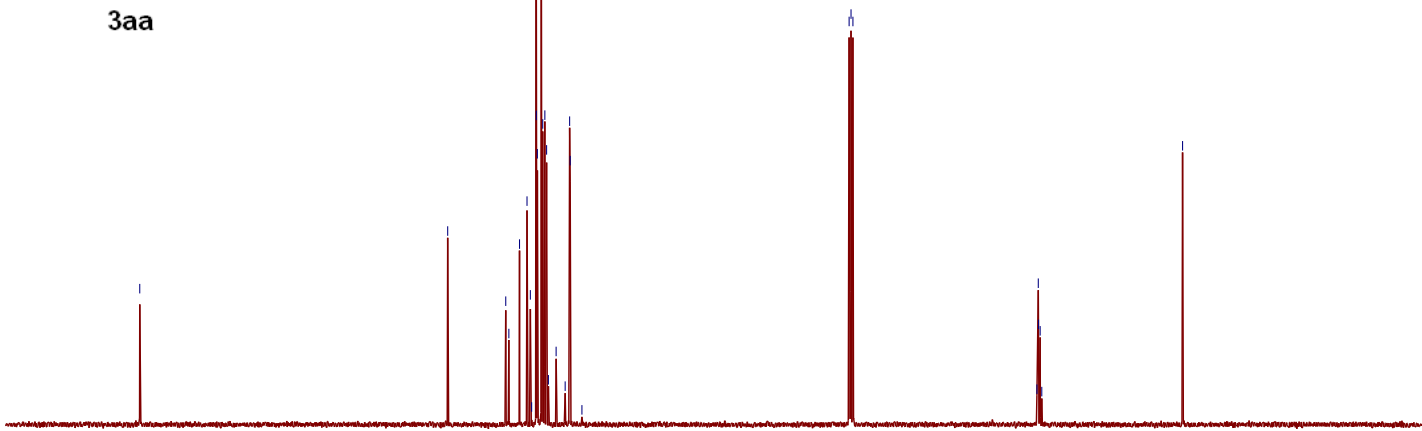

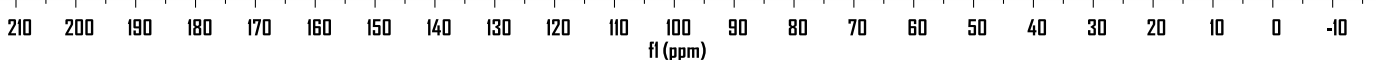




\section{웅몽}

${ }^{19} \mathrm{~F} \mathrm{NMR}\left(376 \mathrm{MHz}, \mathrm{CDCl}_{3}\right)$

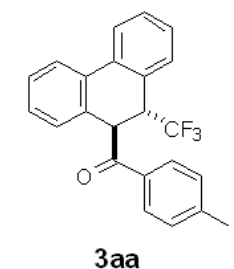

3aa

\begin{tabular}{llllllllllllllllllllllllllllllll}
\hline 20 & 10 & 0 & -10 & -20 & -30 & -40 & -50 & -60 & -70 & -80 & -90 & -100 & -110 & -120 & -130 & -140 & -150 & -160 & -170 & -180 & -190 & -200 & -210 & -220
\end{tabular}

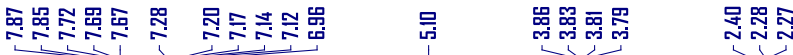

${ }^{1} \mathrm{H}$ NMR $\left(400 \mathrm{MHz}, \mathrm{CDCl}_{3}\right)$

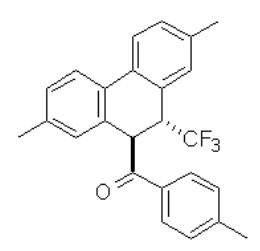

3ab

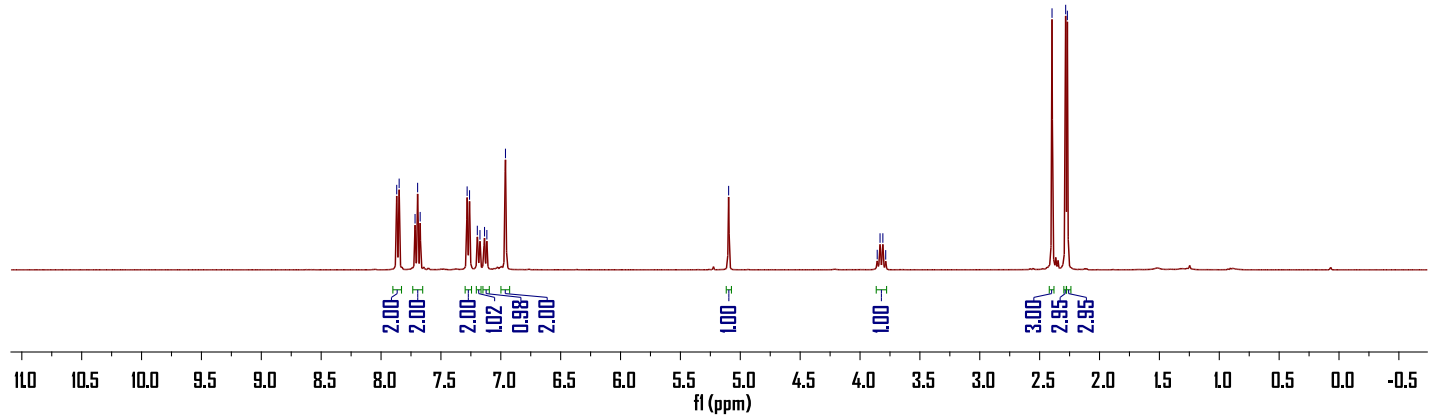


${ }^{13} \mathrm{C} \mathrm{NMR}\left(100 \mathrm{MHz}, \mathrm{CDCl}_{3}\right)$

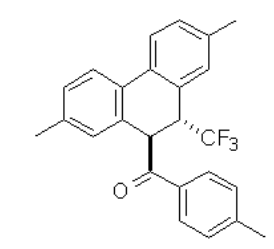

$3 a b$

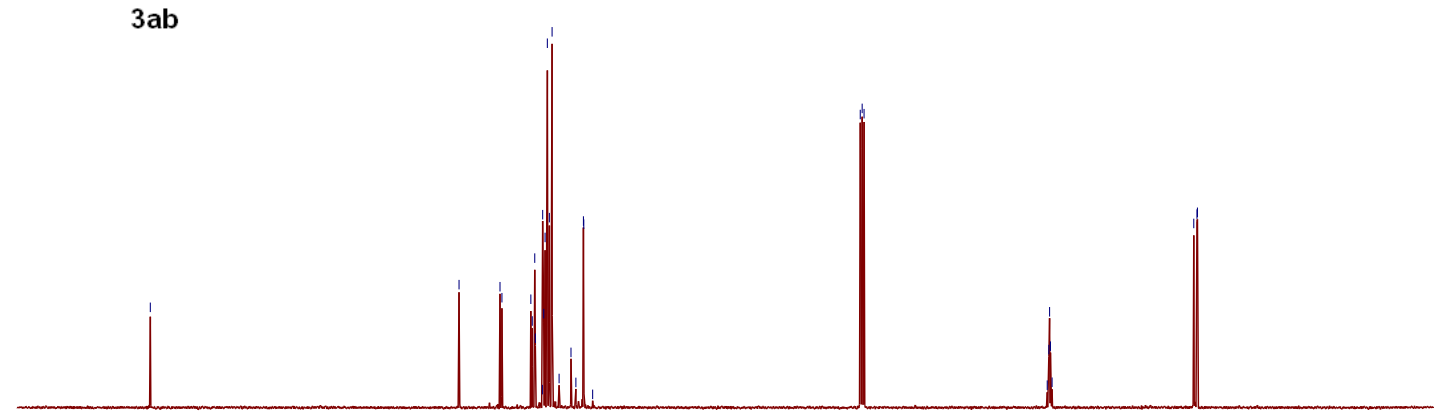

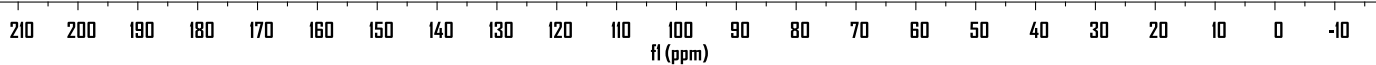

虽言

${ }^{19} \mathrm{~F} \mathrm{NMR}\left(376 \mathrm{MHz}, \mathrm{CDCl}_{3}\right)$

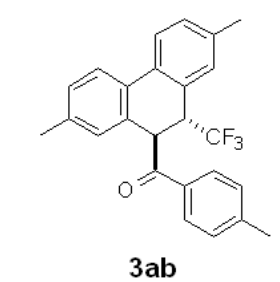

$3 a b$

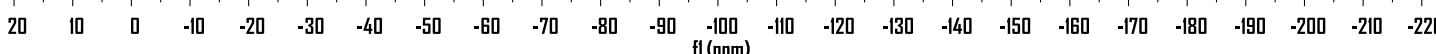




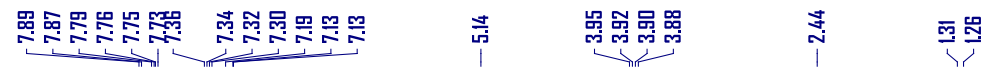

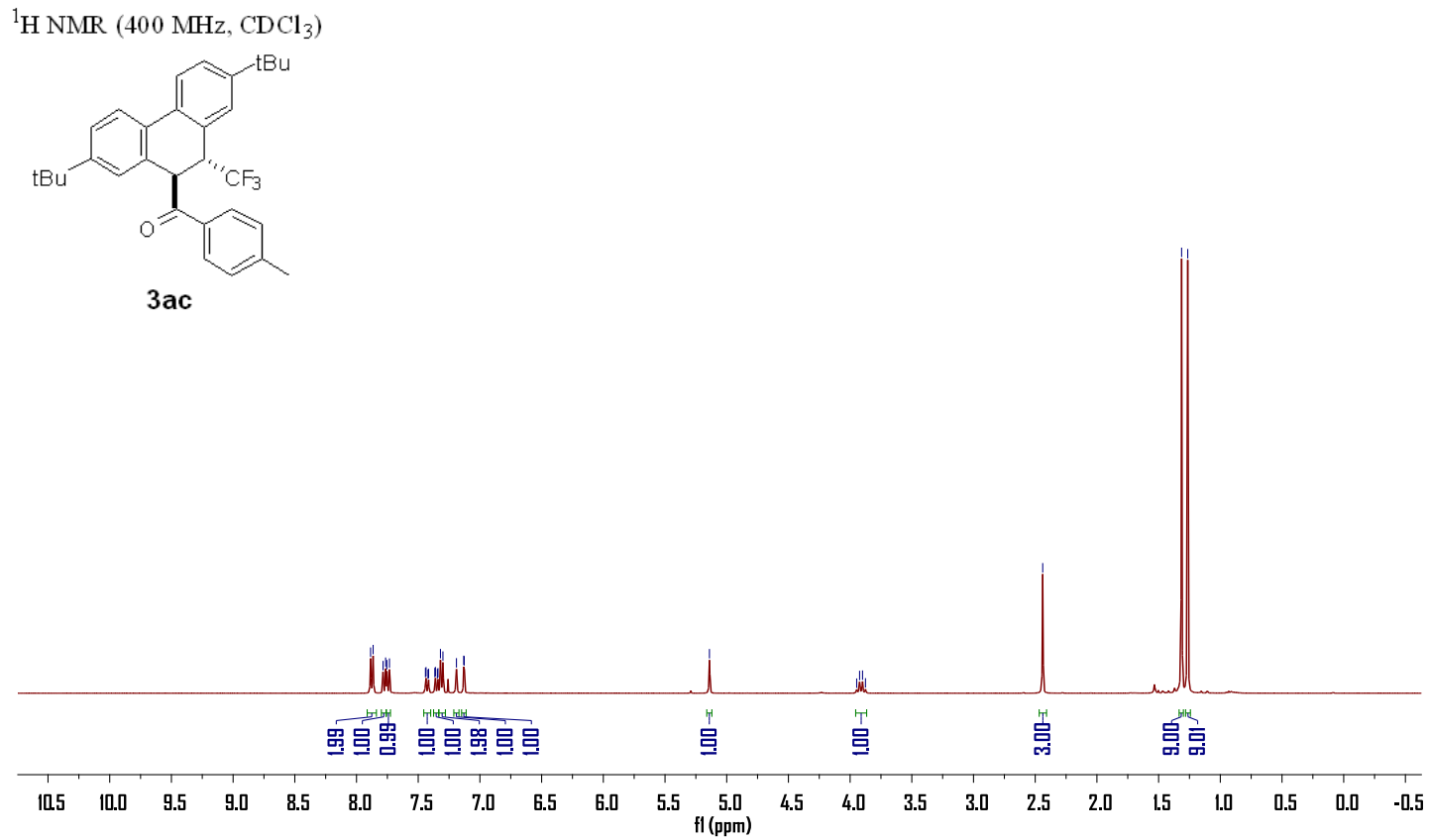

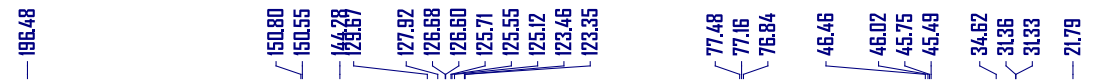

${ }^{13} \mathrm{CNMR}\left(100 \mathrm{MHz}, \mathrm{CDCl}_{3}\right)$

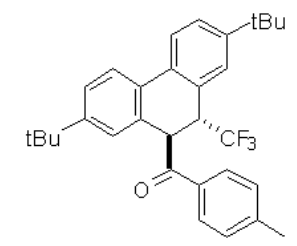

$3 a c$

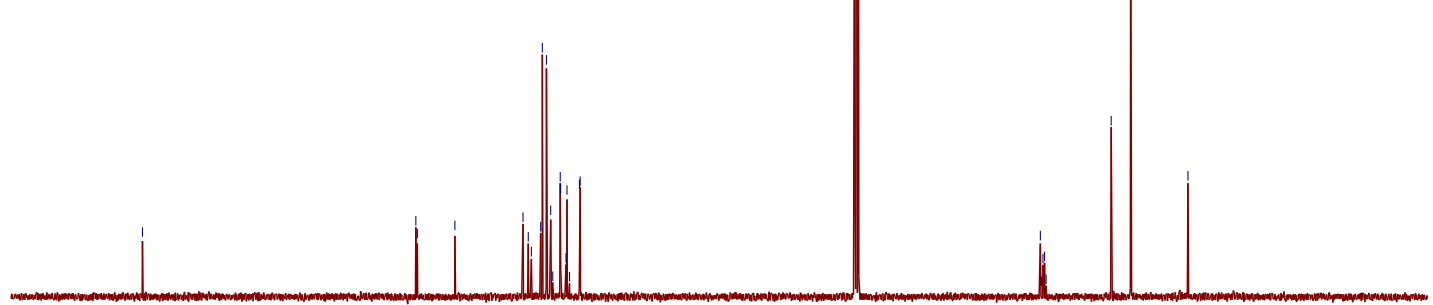

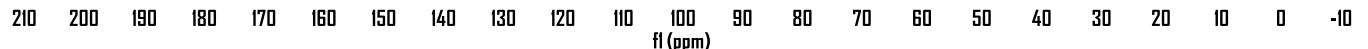


${ }^{19} \mathrm{~F} \mathrm{NMR}\left(565 \mathrm{MHz}, \mathrm{CDCl}_{3}\right)$

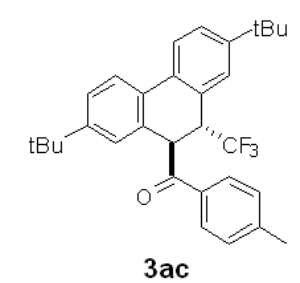

$3 a c$

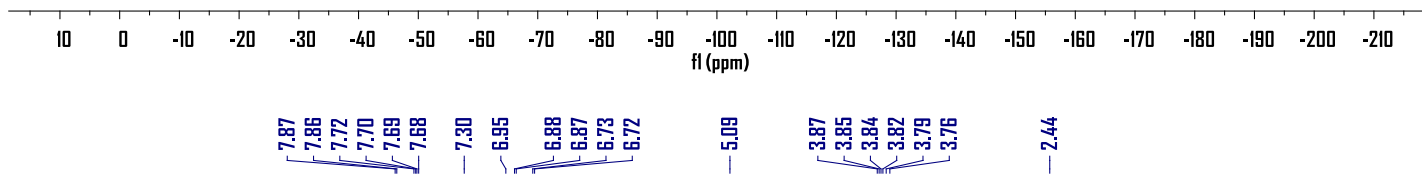

${ }^{1} \mathrm{H}$ NMR $\left(600 \mathrm{MHz}, \mathrm{CDCl}_{3}\right)$
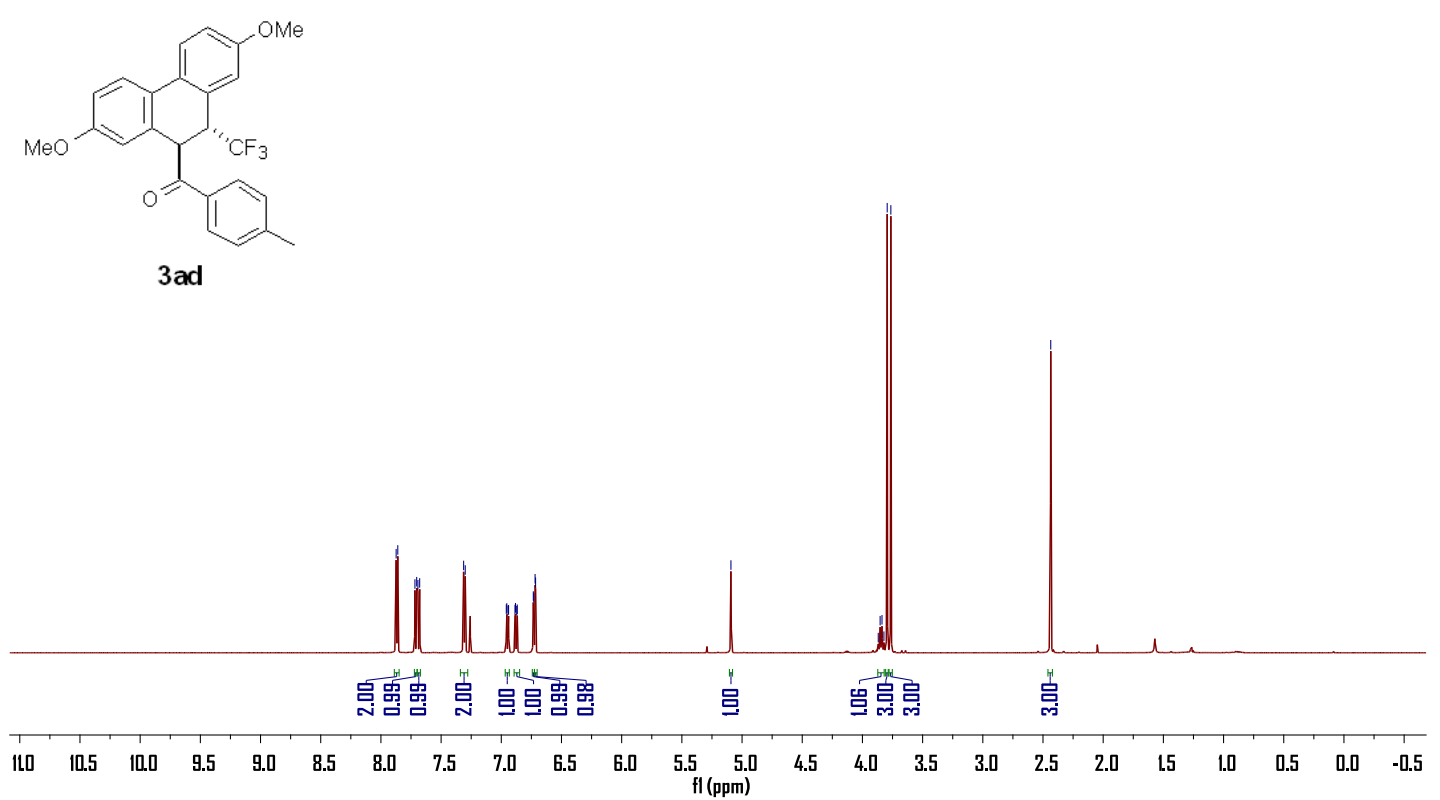


\begin{tabular}{|c|c|c|c|c|}
\hline 㤎嵒 & $\frac{\sqrt[5]{9}}{1}$ & 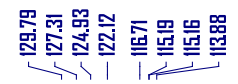 & 哭哭哭 & 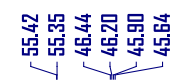 \\
\hline
\end{tabular}

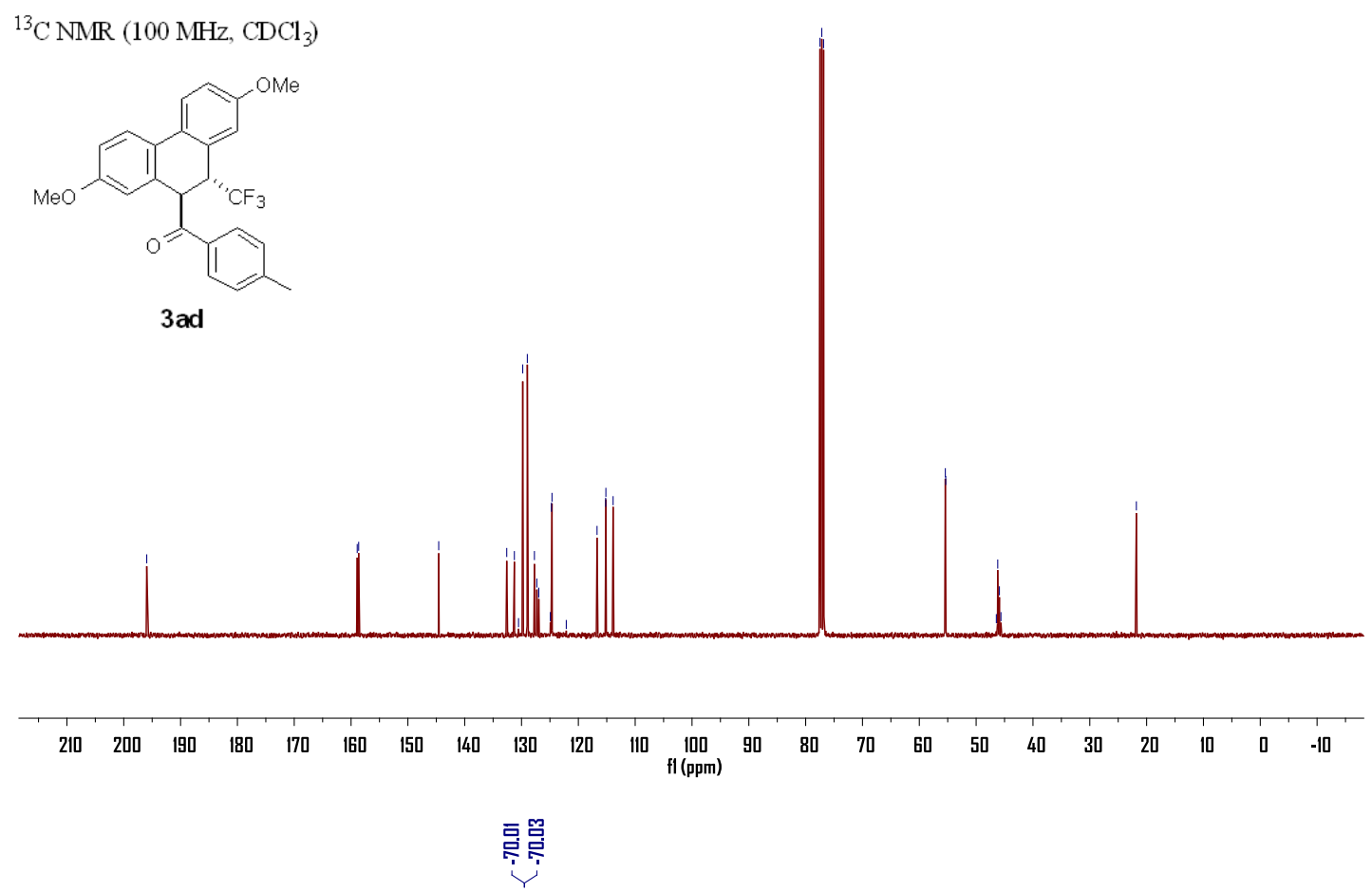

${ }^{19} \mathrm{~F} \mathrm{NMR}\left(376 \mathrm{MHz}, \mathrm{CDCl}_{3}\right)$

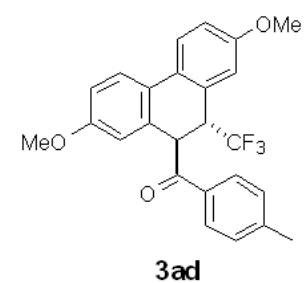

3ad

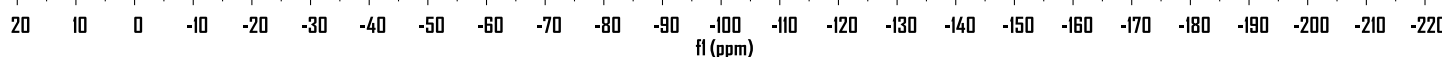




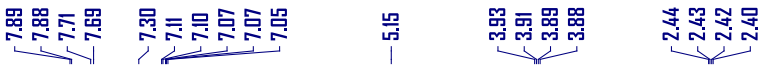

${ }^{1} \mathrm{H} \mathrm{NMR}\left(600 \mathrm{MHz}, \mathrm{CDCl}_{3}\right)$

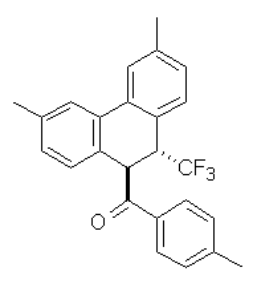

$3 a e$

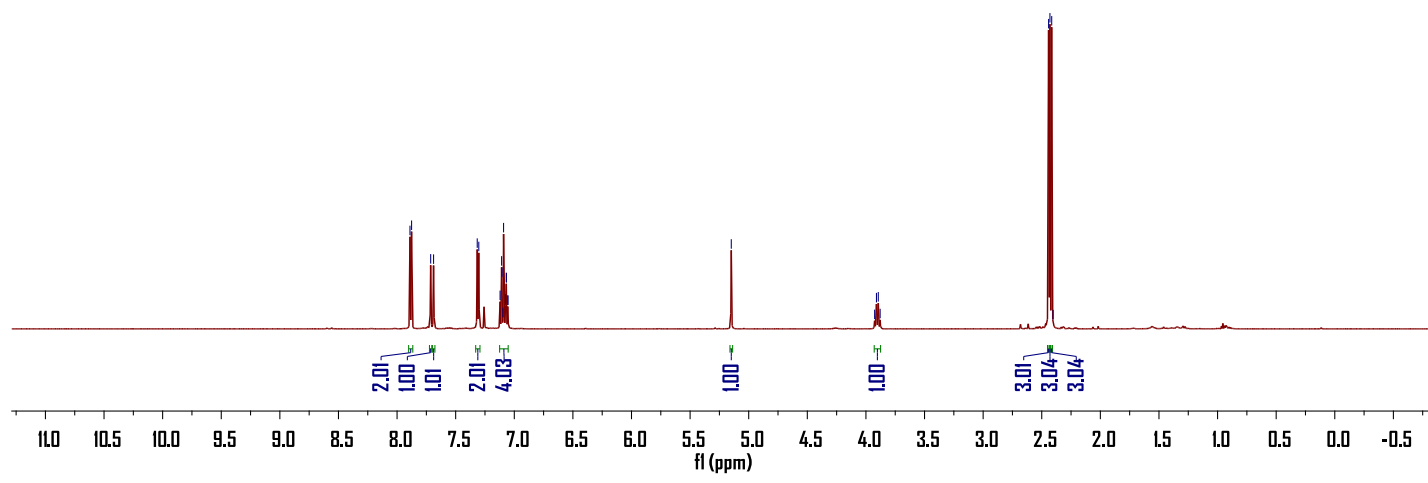

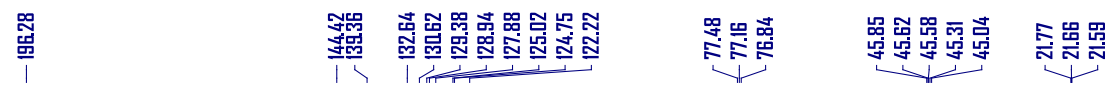

${ }^{13} \mathrm{C} \mathrm{NMR}\left(100 \mathrm{MHz}, \mathrm{CDCl}_{3}\right)$

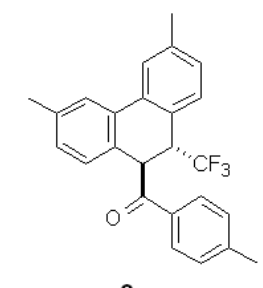

$3 a e$

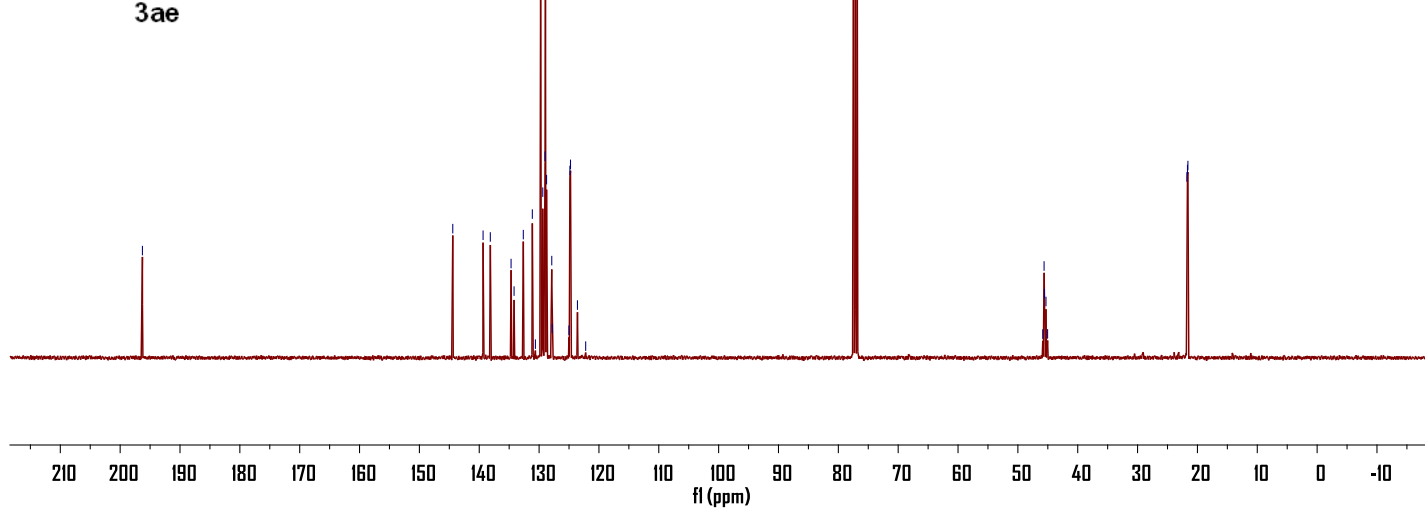


${ }^{19} \mathrm{~F} \mathrm{NMR}\left(565 \mathrm{MHz}, \mathrm{CDCl}_{3}\right)$

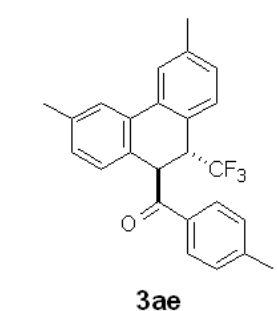

3ae
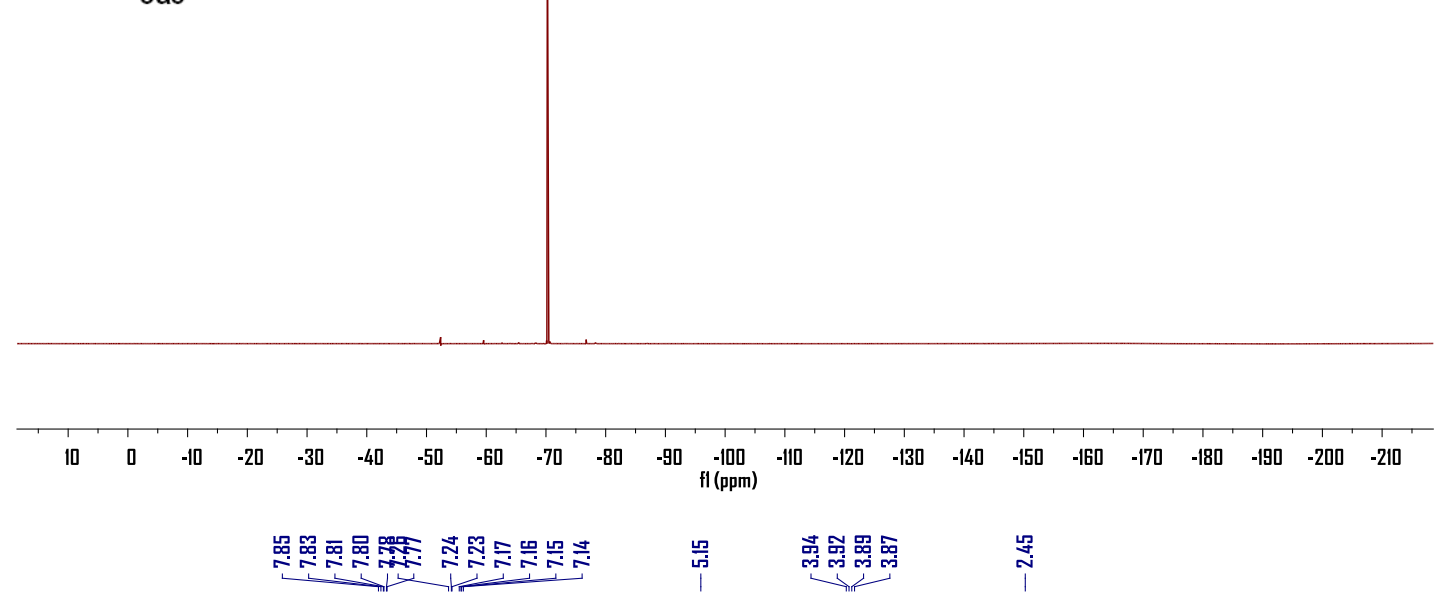

${ }^{1} \mathrm{H}$ NMR $\left(400 \mathrm{MHz}, \mathrm{CDCl}_{3}\right)$

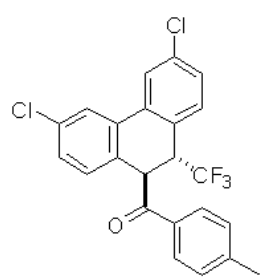

3af

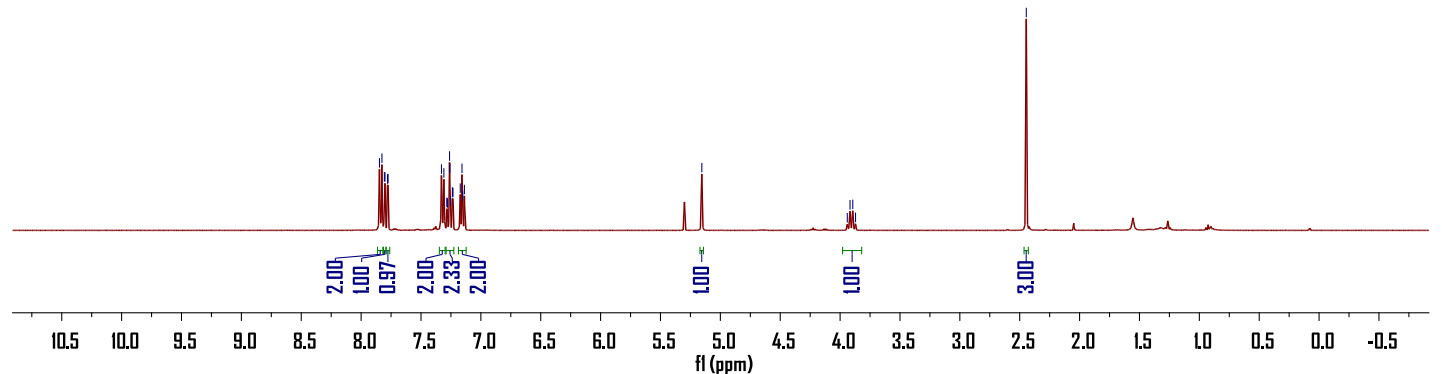


${ }^{13} \mathrm{C} \mathrm{NMR}\left(100 \mathrm{MHz}, \mathrm{CDCl}_{3}\right)$

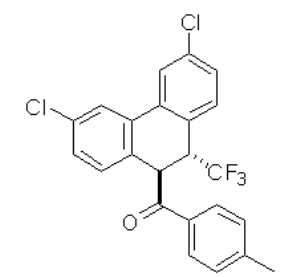

3af

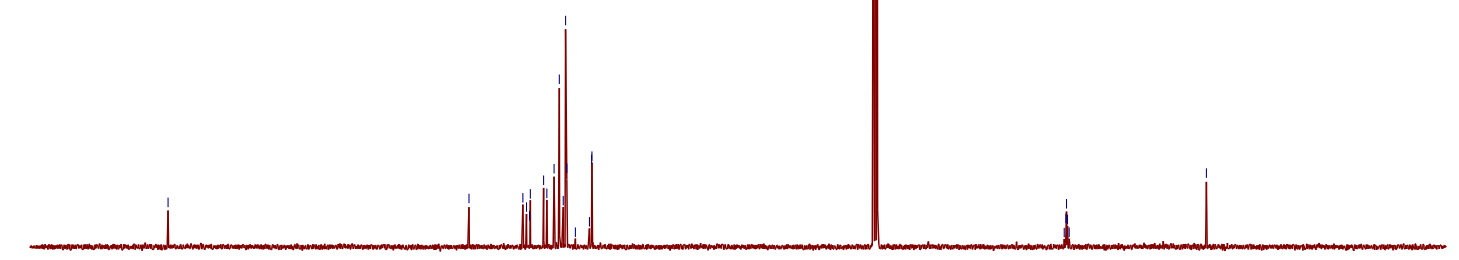

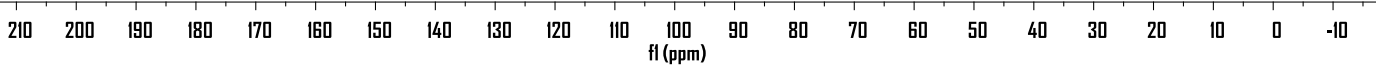

芛

${ }^{19} \mathrm{~F} \mathrm{NMR}\left(376 \mathrm{MHz}, \mathrm{CDCl}_{3}\right)$

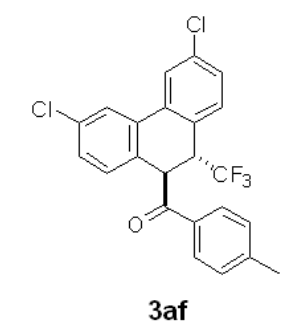

3af

\begin{tabular}{lllllllllllllllllllllllllllllll}
\hline 20 & 10 & 0 & -10 & -20 & -30 & -40 & -50 & -60 & -70 & -80 & -90 & -100 & -110 & -120 & -130 & -140 & -150 & -160 & -170 & -180 & -190 & -200 & -210 & -220
\end{tabular} 


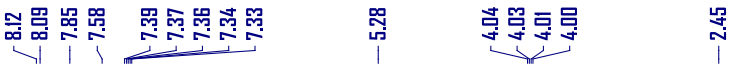

${ }^{1} \mathrm{H}$ NMR $\left(600 \mathrm{MHz}, \mathrm{CDCl}_{3}\right)$

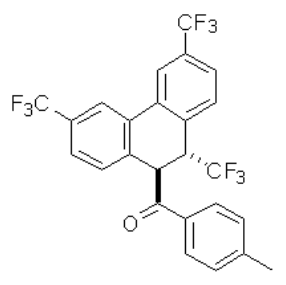

3ag

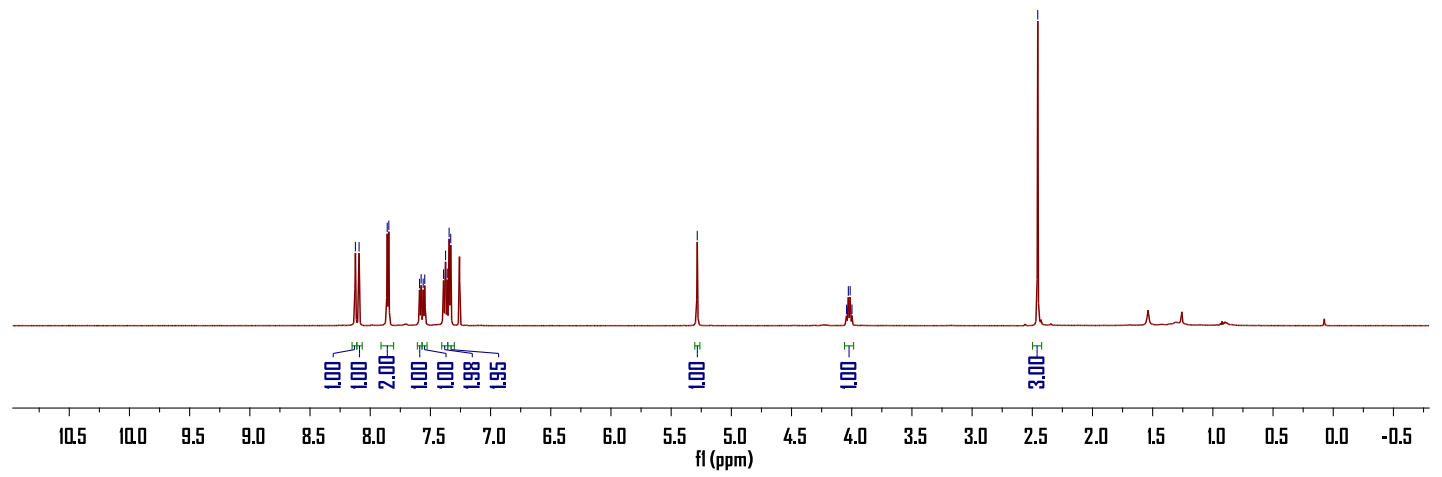

范

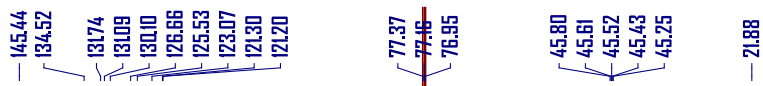

${ }^{13} \mathrm{C} \mathrm{NMR}\left(150 \mathrm{MHz}, \mathrm{CDCl}_{3}\right)$

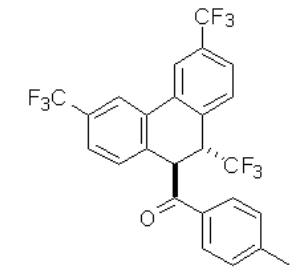

3 ag

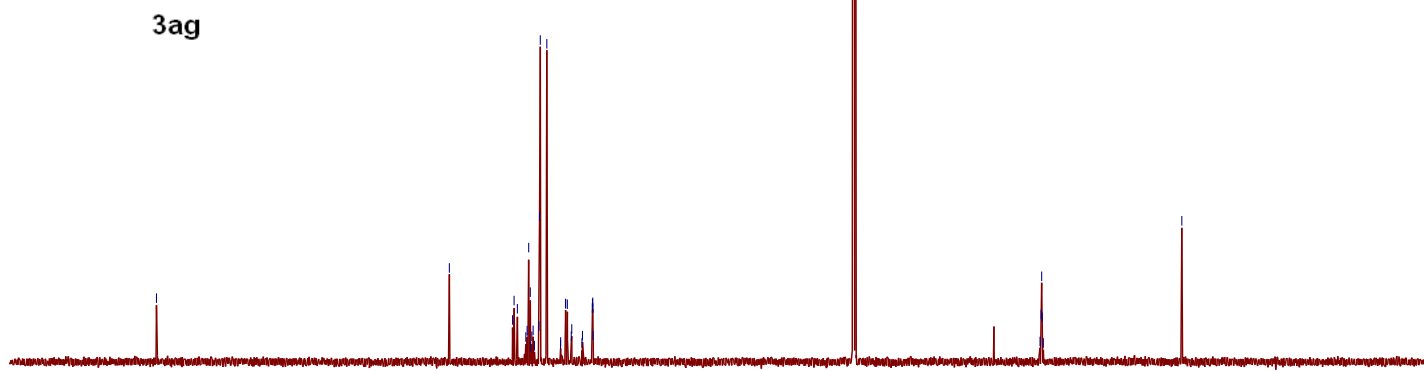

$\begin{array}{lllllllllllllllllllllll}210 & 200 & 190 & 180 & 170 & 160 & 150 & 140 & 130 & 120 & 110 & 100 & 90 & 80 & 70 & 60 & 50 & 40 & 30 & 20 & 10 & 0 & -10\end{array}$ 


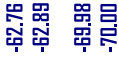

${ }^{19} \mathrm{~F} \mathrm{NMR}\left(376 \mathrm{MHz}, \mathrm{CDCl}_{3}\right)$<smiles>Cc1ccc(C(=O)C2c3ccc(C(F)(F)F)cc3-c3cc(C(F)(F)F)ccc3C2C(F)(F)F)cc1</smiles>

3ag

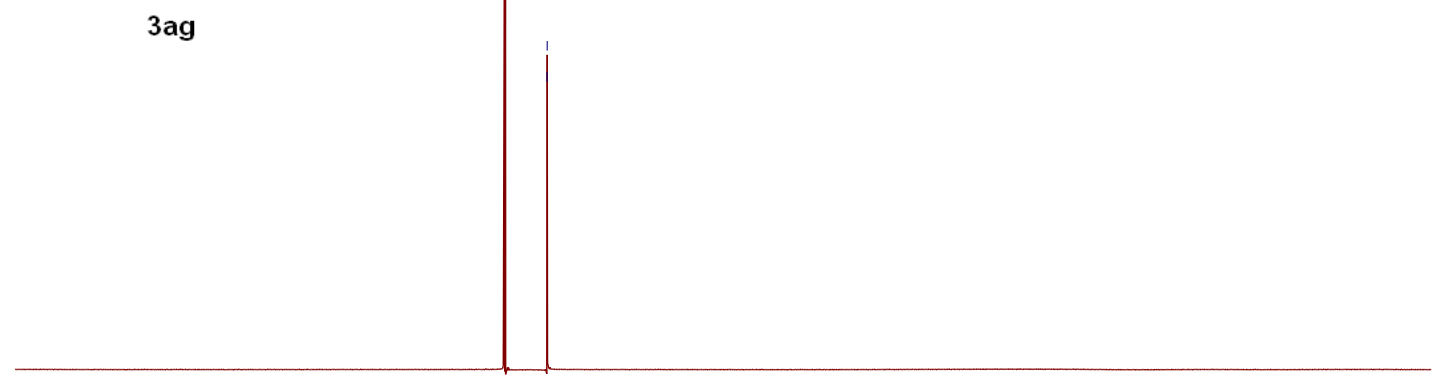

\begin{tabular}{lllllllllllllllllllllllllllllll}
\hline 20 & 10 & 0 & -10 & -20 & -30 & -40 & -50 & -60 & -70 & -80 & -90 & -100 & -110 & -120 & -130 & -140 & -150 & -160 & -170 & -180 & -190 & -200 & -210 & -220
\end{tabular}

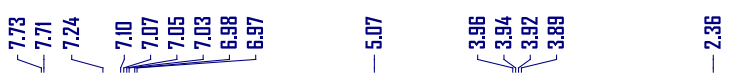

${ }^{1} \mathrm{H} \mathrm{NMR}\left(400 \mathrm{MHz}, \mathrm{CDCl}_{3}\right)$

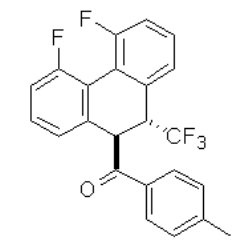

3ah

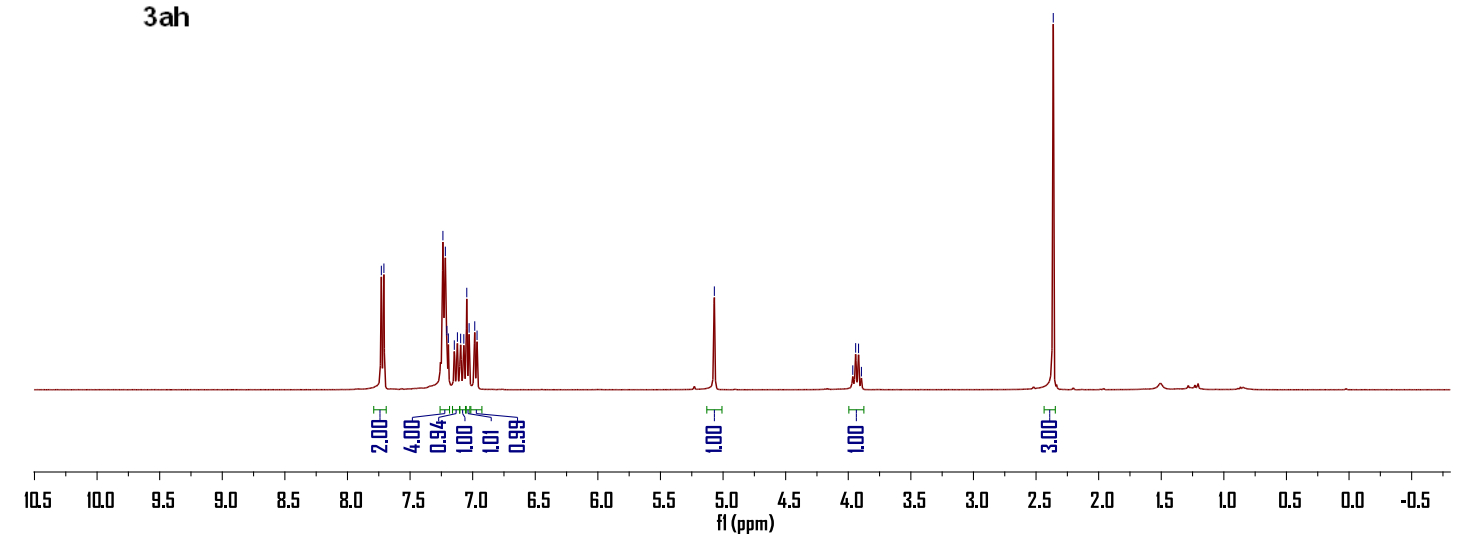




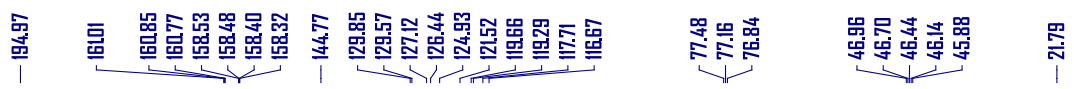

${ }^{13} \mathrm{C} \mathrm{NMR}\left(100 \mathrm{MHz}, \mathrm{CDCl}_{3}\right)$

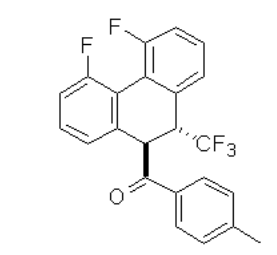

3ah

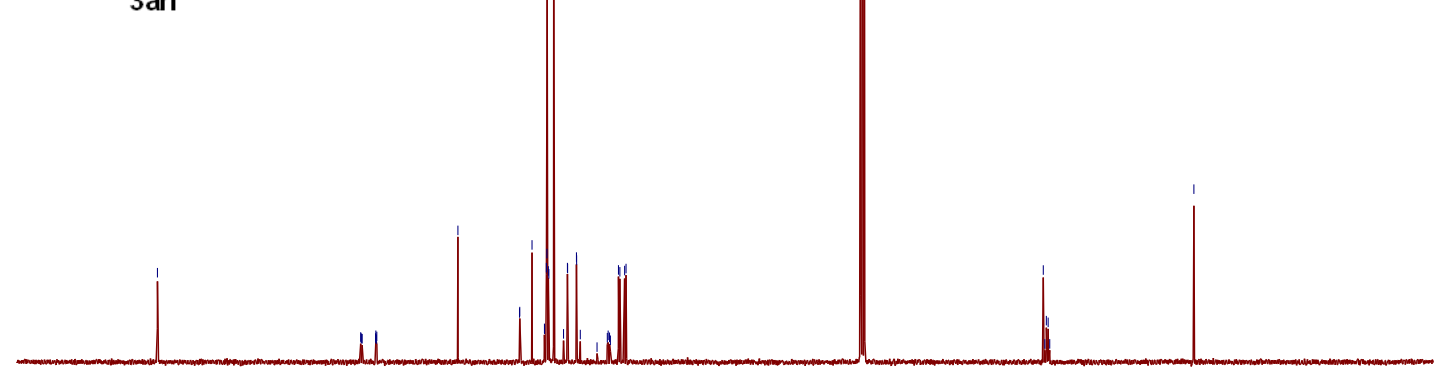

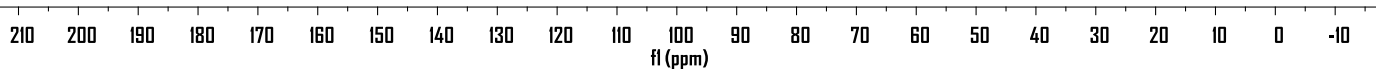

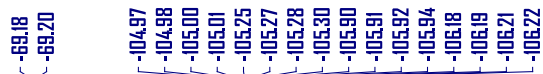

${ }^{19} \mathrm{~F} \mathrm{NMR}\left(376 \mathrm{MHz}, \mathrm{CDCl}_{3}\right)$

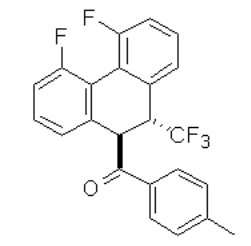

3ah

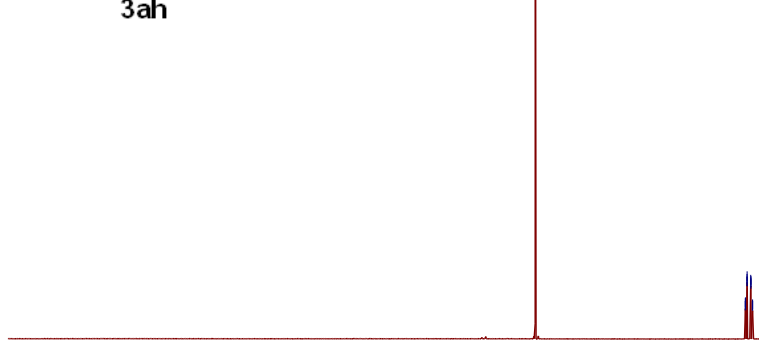

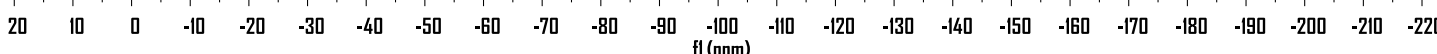




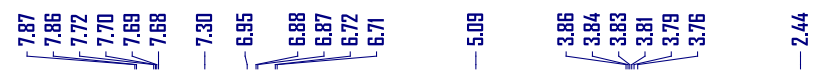

${ }^{1} \mathrm{H} \mathrm{NMR}\left(600 \mathrm{MHz}, \mathrm{CDCl}_{3}\right)$

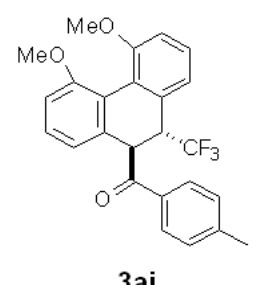

3 ai

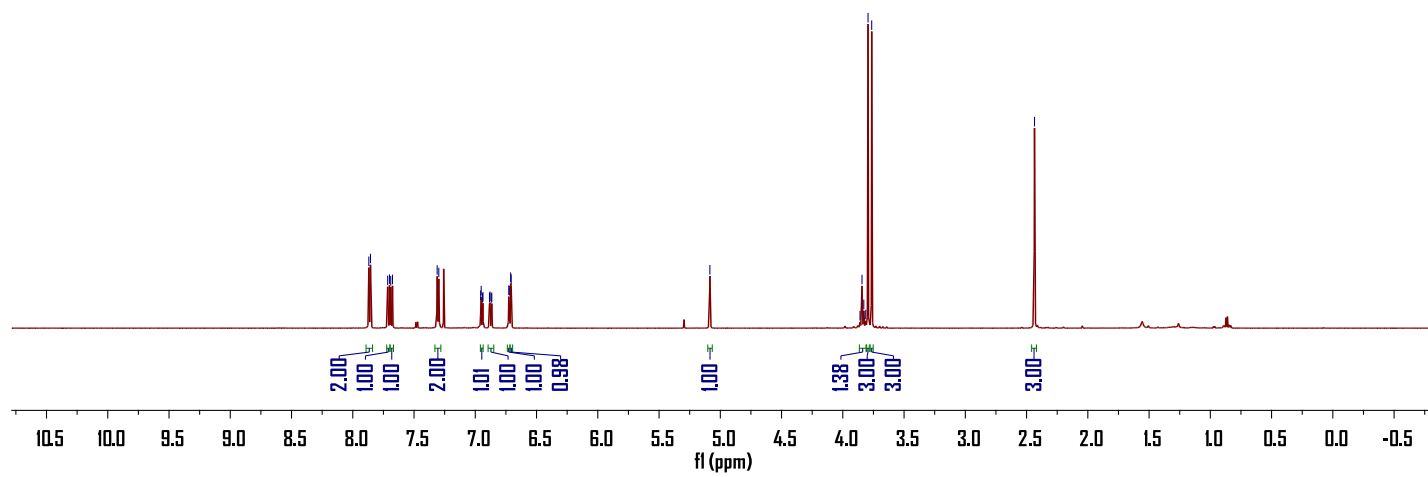

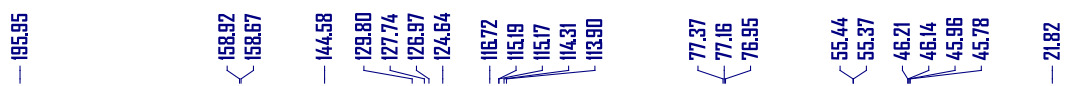

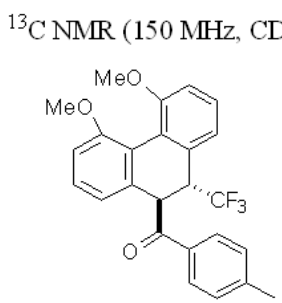

3ai

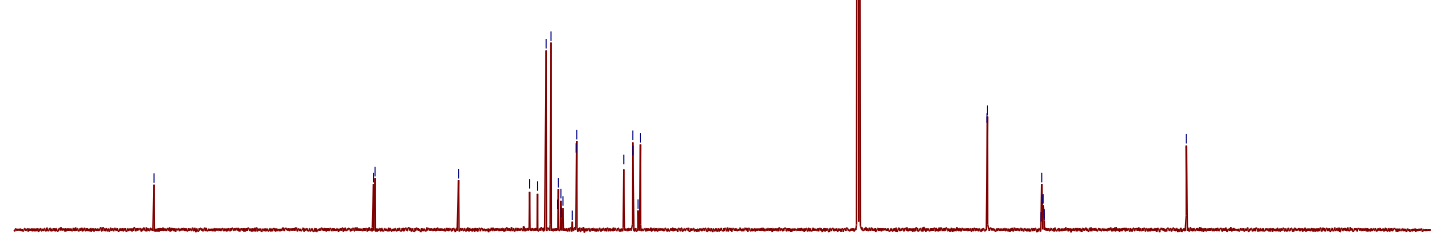

$\begin{array}{lllllllllllllllllllllll}210 & 200 & 190 & 180 & 170 & 160 & 150 & 140 & 130 & 120 & 110 & \underset{f l}{100}(\mathrm{ppm}) & 90 & 80 & 70 & 60 & 50 & 40 & 30 & 20 & 10 & 0 & -10\end{array}$ 


\section{总总}

${ }^{19} \mathrm{~F} \mathrm{NMR}\left(376 \mathrm{MHz}, \mathrm{CDCl}_{3}\right)$

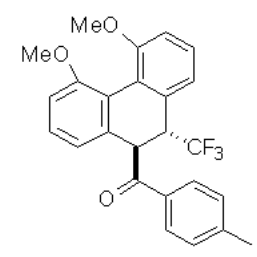

3ai

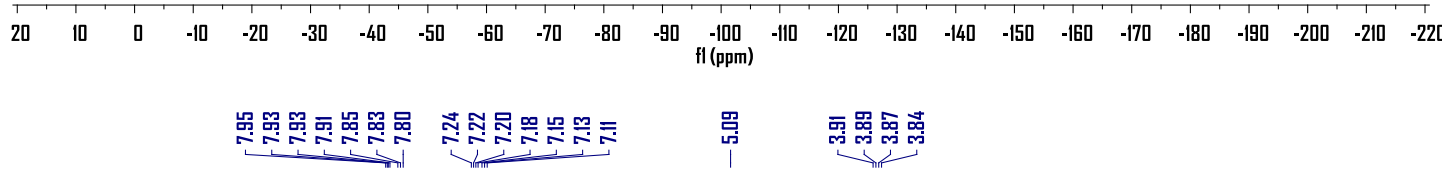

${ }^{1} \mathrm{H}$ NMR $\left(400 \mathrm{MHz}, \mathrm{CDCl}_{3}\right)$<smiles>O=C(c1ccc(F)cc1)C1c2ccccc2-c2ccccc2C1C(F)(F)F</smiles>

3ba

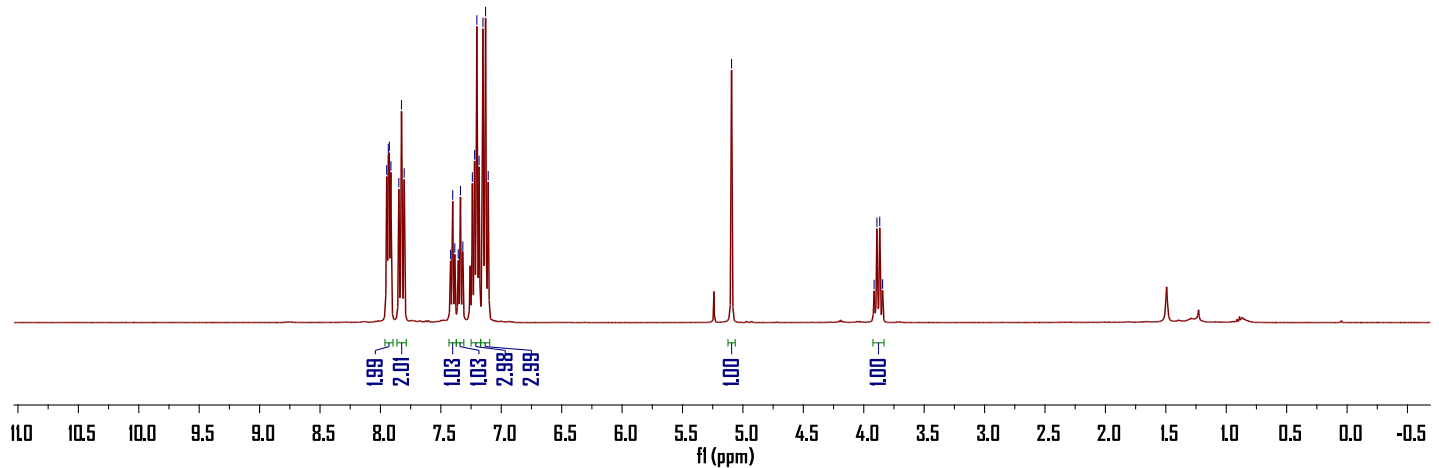




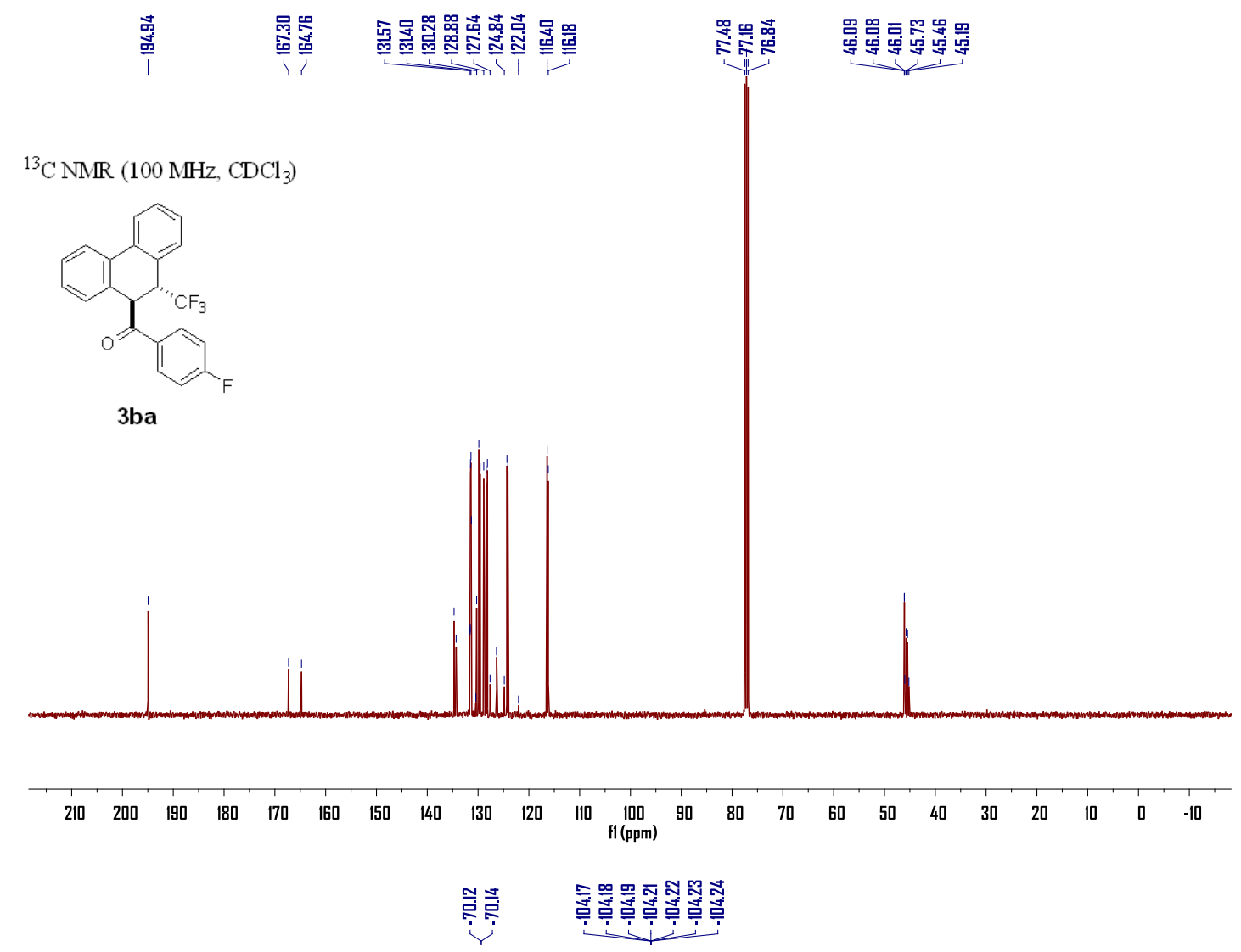

${ }^{19} \mathrm{~F} \mathrm{NMR}\left(376 \mathrm{MHz}, \mathrm{CDCl}_{3}\right)$

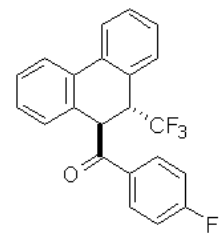

3 ba

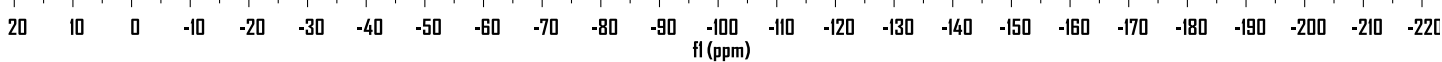




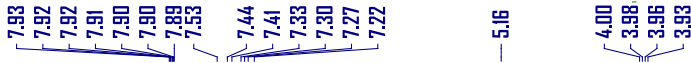

${ }^{1} \mathrm{H}$ NMR $\left(400 \mathrm{MHz}, \mathrm{CDCl}_{3}\right)$

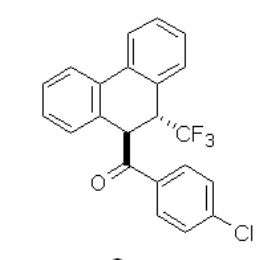

$3 \mathrm{ca}$

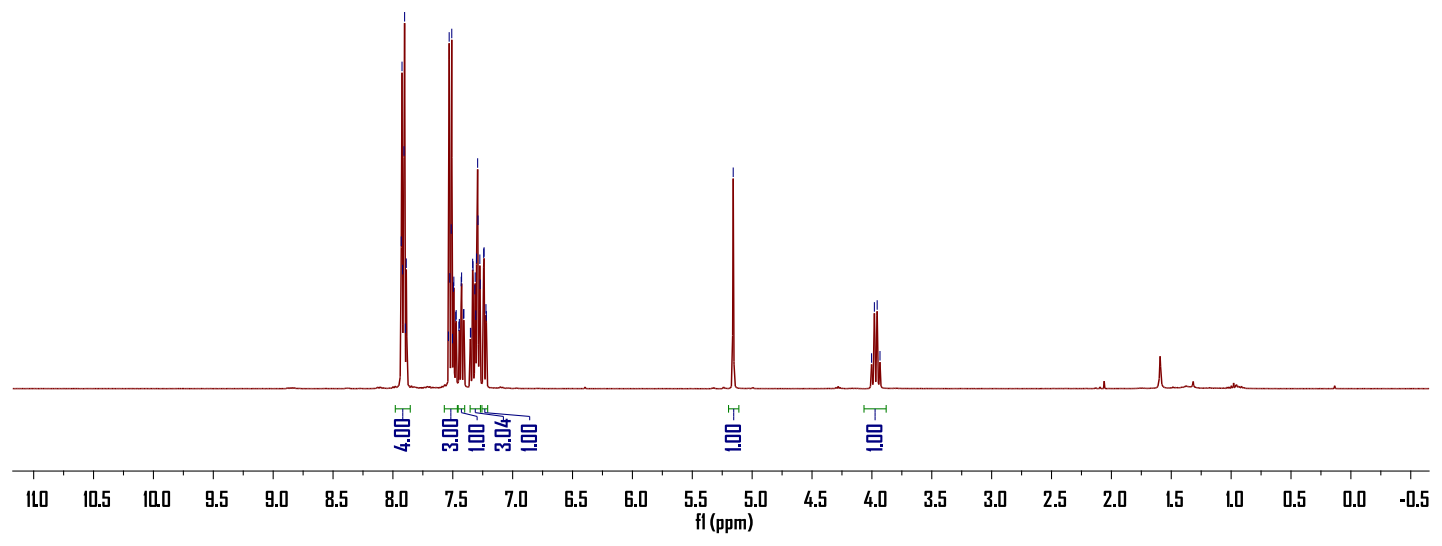

営

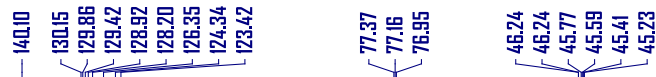

${ }^{13} \mathrm{C} \mathrm{NMR}\left(150 \mathrm{MHz}, \mathrm{CDCl}_{3}\right)$

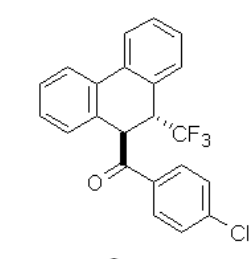

$3 c a$

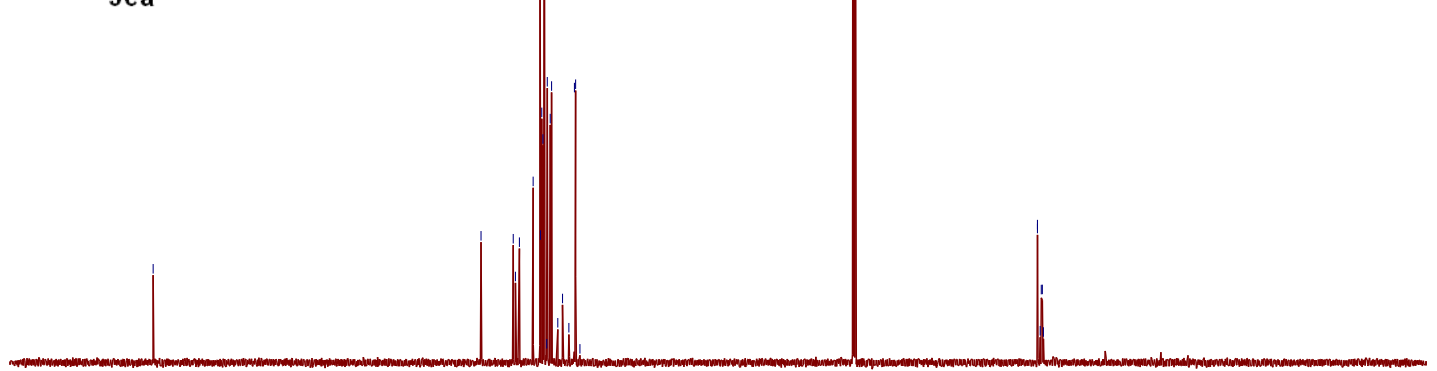

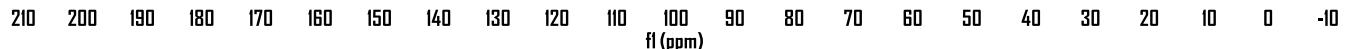


${ }^{19} \mathrm{~F} \mathrm{NMR}\left(376 \mathrm{MHz}, \mathrm{CDCl}_{3}\right)$

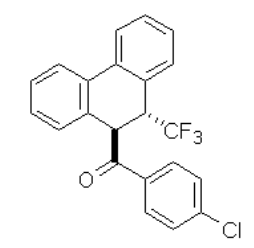

3ca

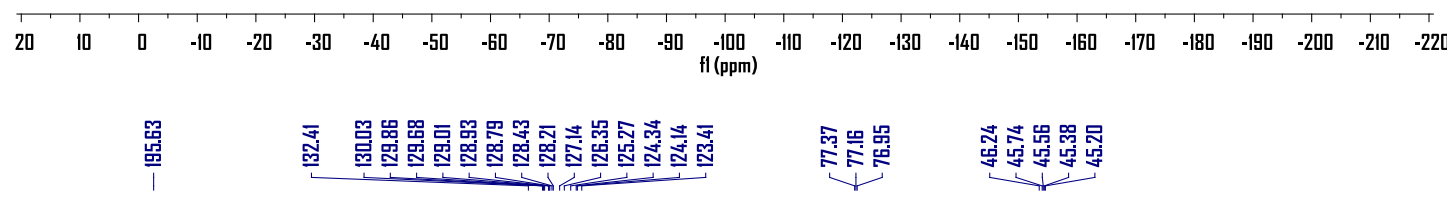

${ }^{13} \mathrm{CNMR}\left(150 \mathrm{MHz}, \mathrm{CDCl}_{3}\right)$

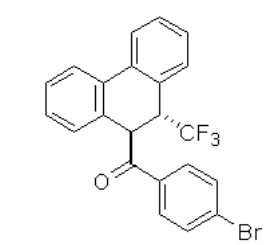

3da

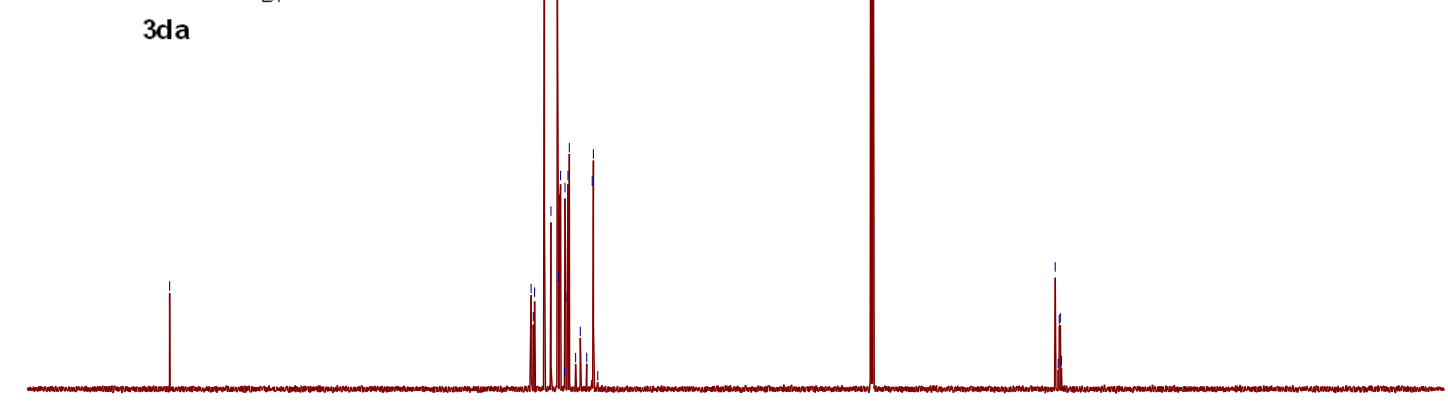

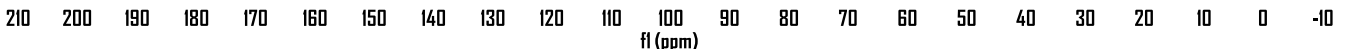




\section{员器}

${ }^{19} \mathrm{~F} \mathrm{NMR}\left(376 \mathrm{MHz}, \mathrm{CDCl}_{3}\right)$

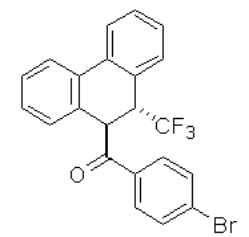

3da

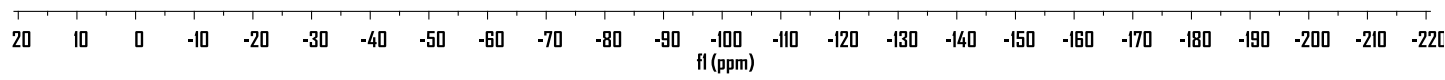

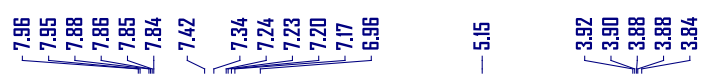

${ }^{1} \mathrm{H} \mathrm{NMR}\left(600 \mathrm{MHz}, \mathrm{CDCl}_{3}\right)$

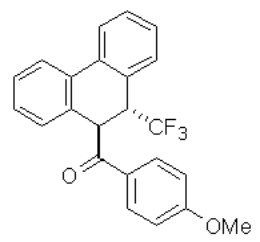

3ea

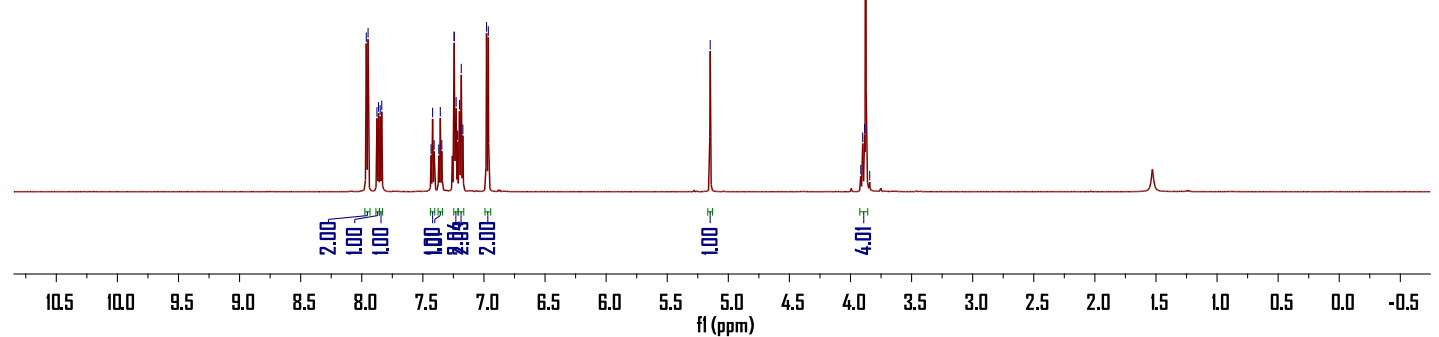




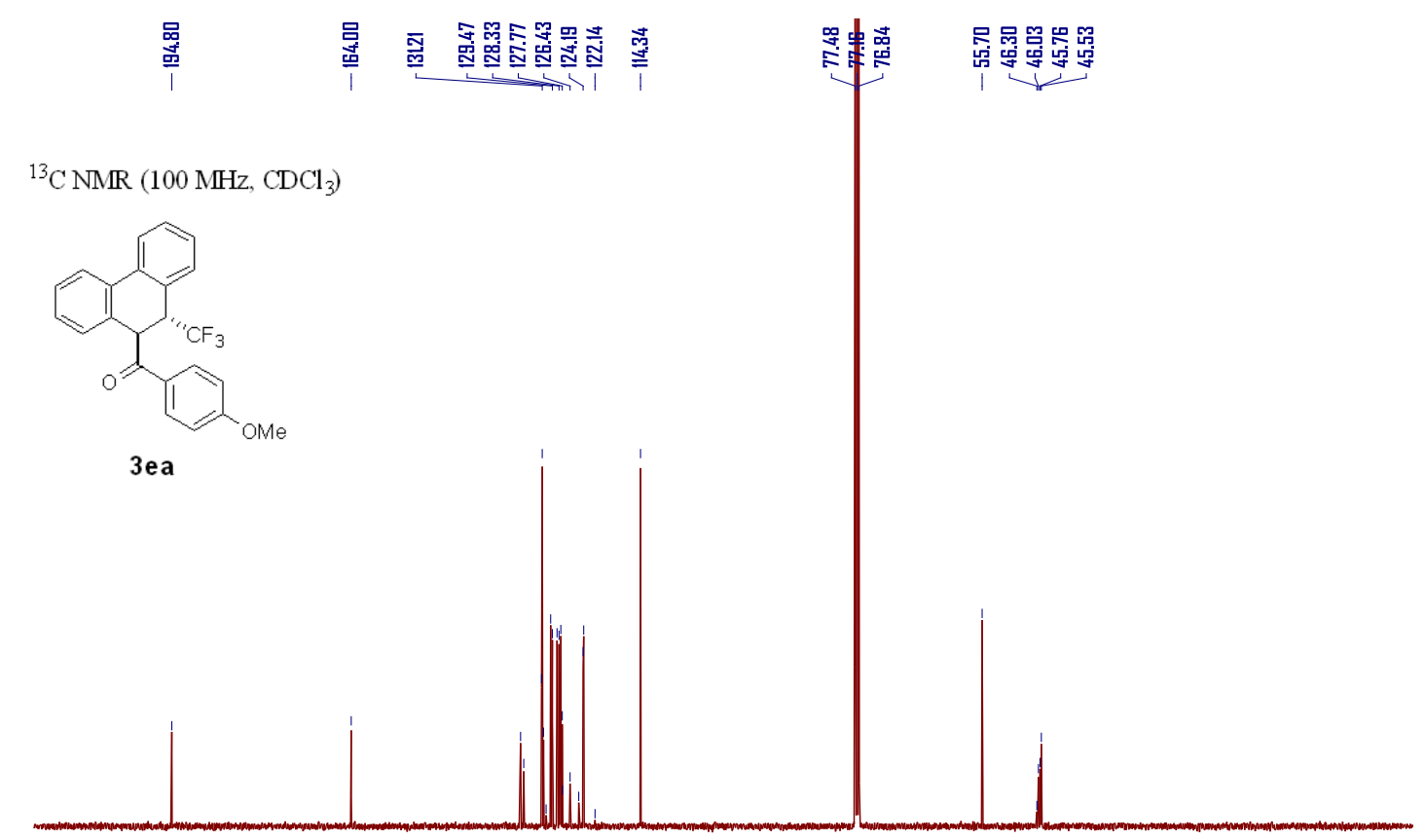

$\begin{array}{llllllllllllllllllllllllll}210 & 200 & 190 & 180 & 170 & 160 & 150 & 140 & 130 & 120 & 110 & \begin{array}{c}100 \\ \mathrm{fl}(\mathrm{ppm})\end{array} & 90 & 80 & 70 & 60 & 50 & 40 & 30 & 20 & 10 & 0 & -10\end{array}$

\section{롤}

${ }^{19} \mathrm{~F} \mathrm{NMR}\left(565 \mathrm{MHz}, \mathrm{CDCl}_{3}\right.$ )

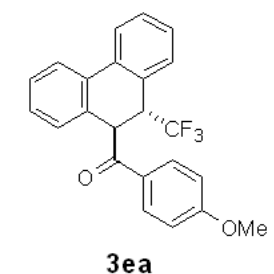

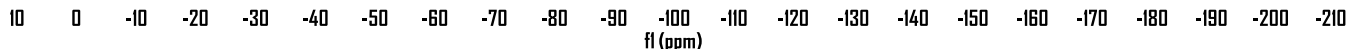




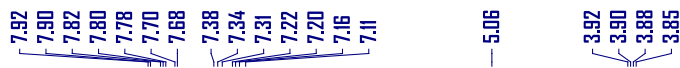

${ }^{1} \mathrm{H} \mathrm{NMR}\left(400 \mathrm{MHz}, \mathrm{CDCl}_{3}\right)$

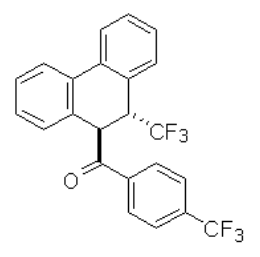

$3 \mathbf{f a}$

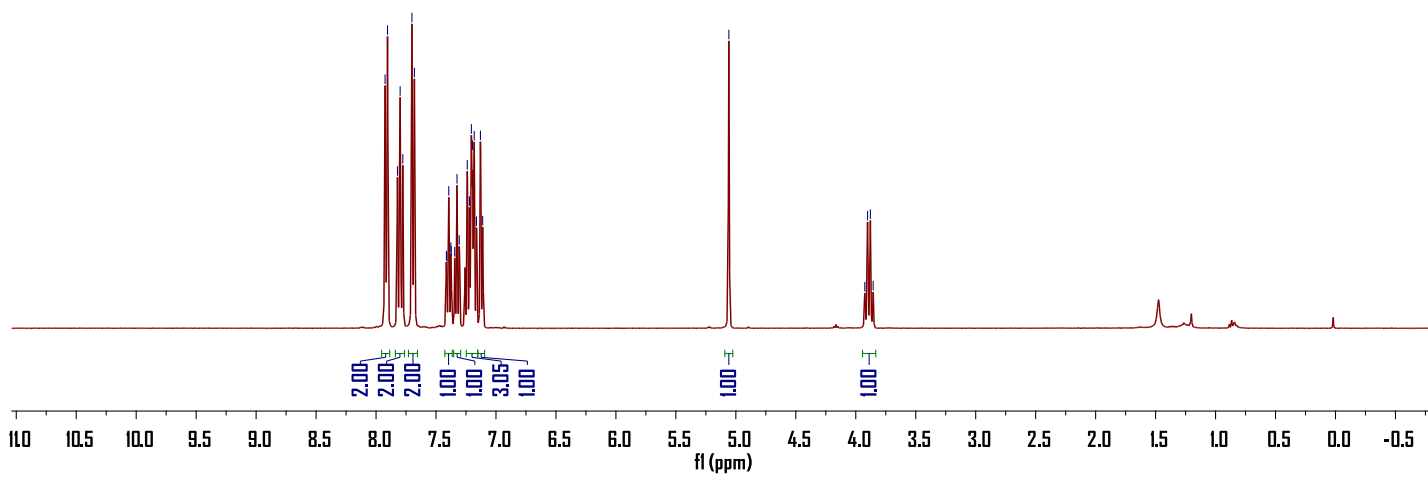

品 骂

${ }^{13} \mathrm{C} \mathrm{NMR}\left(150 \mathrm{MHz}, \mathrm{CDCl}_{3}\right)$

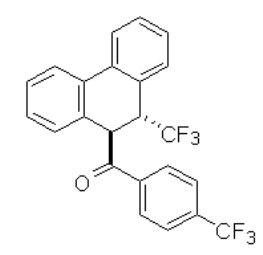

$3 f a$

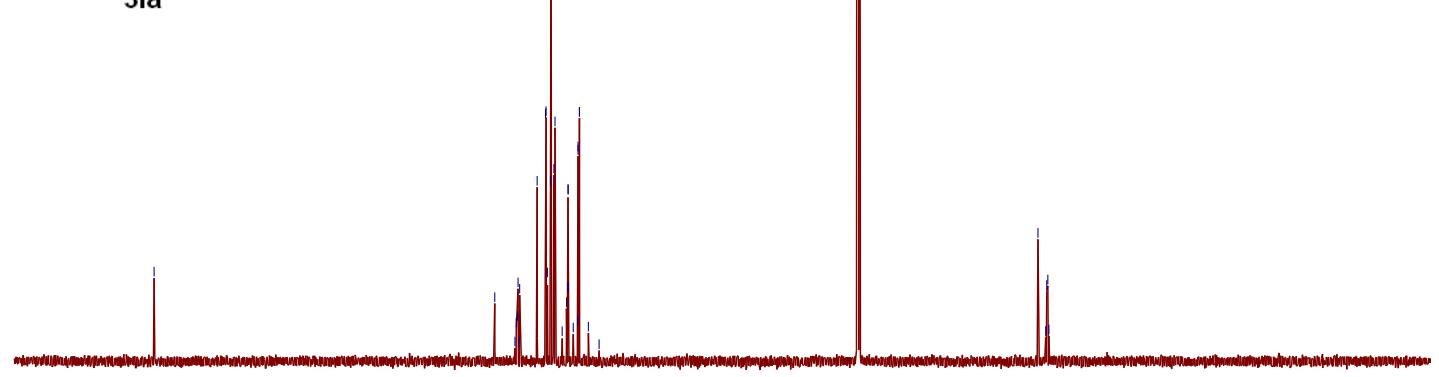

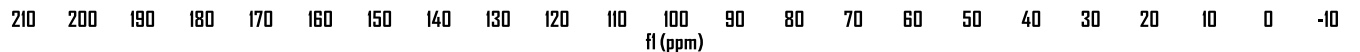




\section{哭 喜总}

${ }^{19} \mathrm{~F} \mathrm{NMR}\left(376 \mathrm{MHz}, \mathrm{CDCl}_{3}\right)$

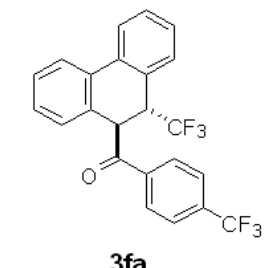

3 a

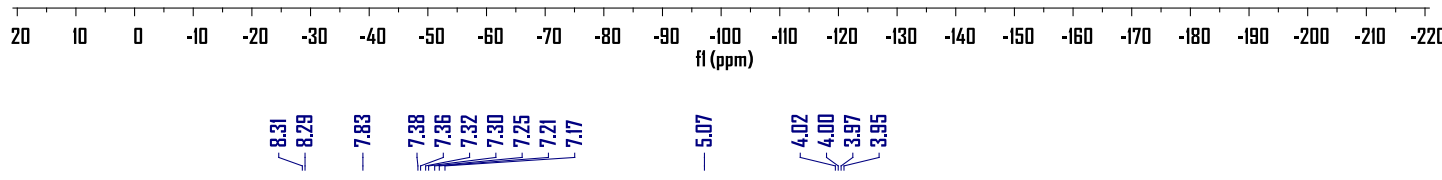

${ }^{1} \mathrm{H}$ NMR $\left(400 \mathrm{MHz}, \mathrm{CDCl}_{3}\right)$

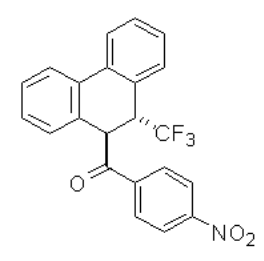

3ga

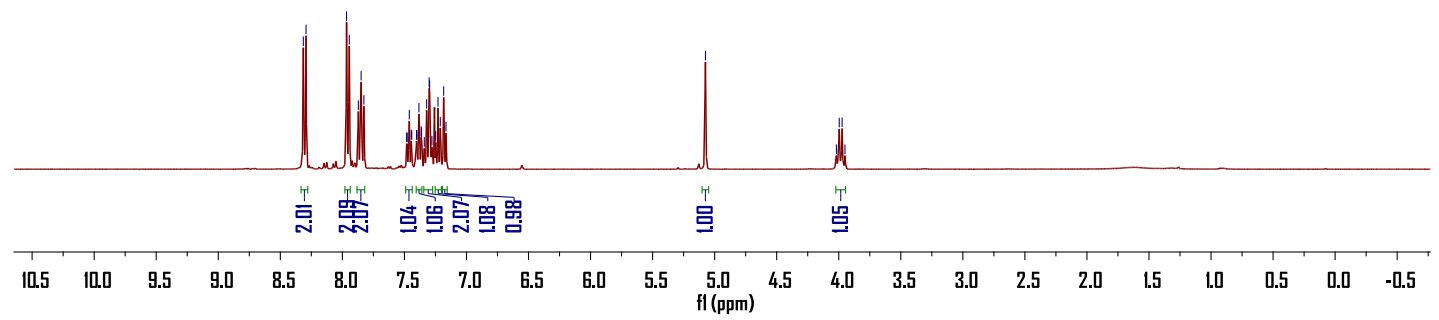


${ }^{13} \mathrm{C} \mathrm{NMR}\left(100 \mathrm{MHz}, \mathrm{CDCl}_{3}\right)$

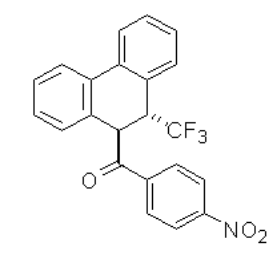

3ga

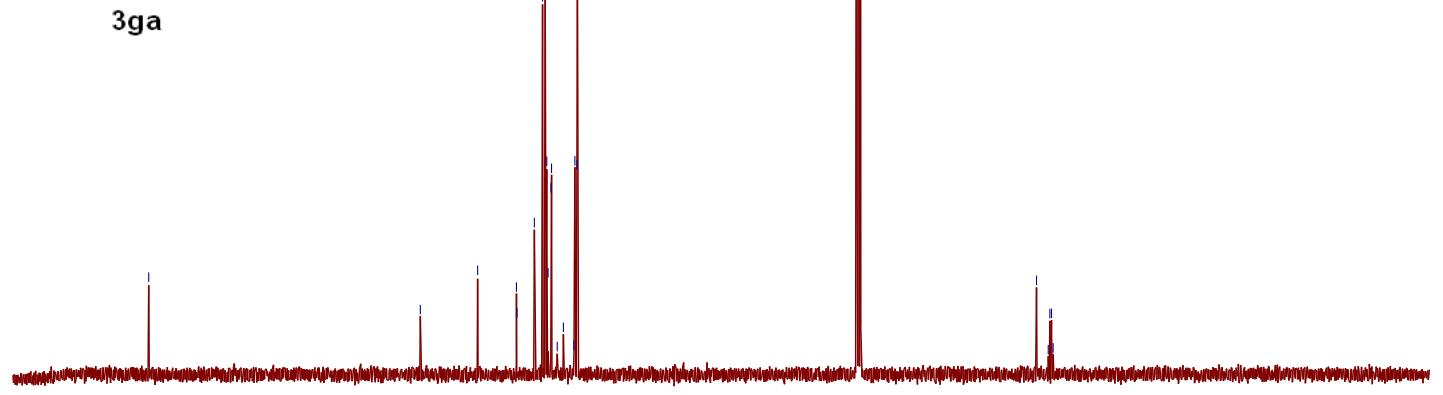

$\begin{array}{lllllllllllllllllllllllllllllll}210 & 200 & 190 & 180 & 170 & 160 & 150 & 140 & 130 & 120 & 110 & \begin{array}{c}100 \\ \mathrm{fl}(\mathrm{ppm})\end{array} & 90 & 80 & 70 & 60 & 50 & 40 & 30 & 20 & 10 & 0 & -10\end{array}$

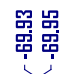

${ }^{19} \mathrm{~F} \mathrm{NMR}\left(376 \mathrm{MHz}, \mathrm{CDCl}_{3}\right)$

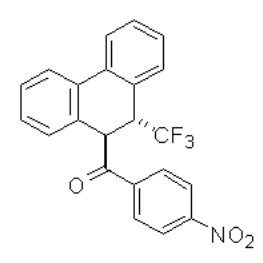

3ga

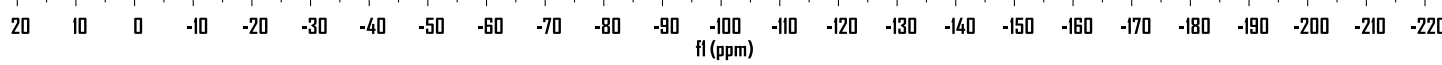



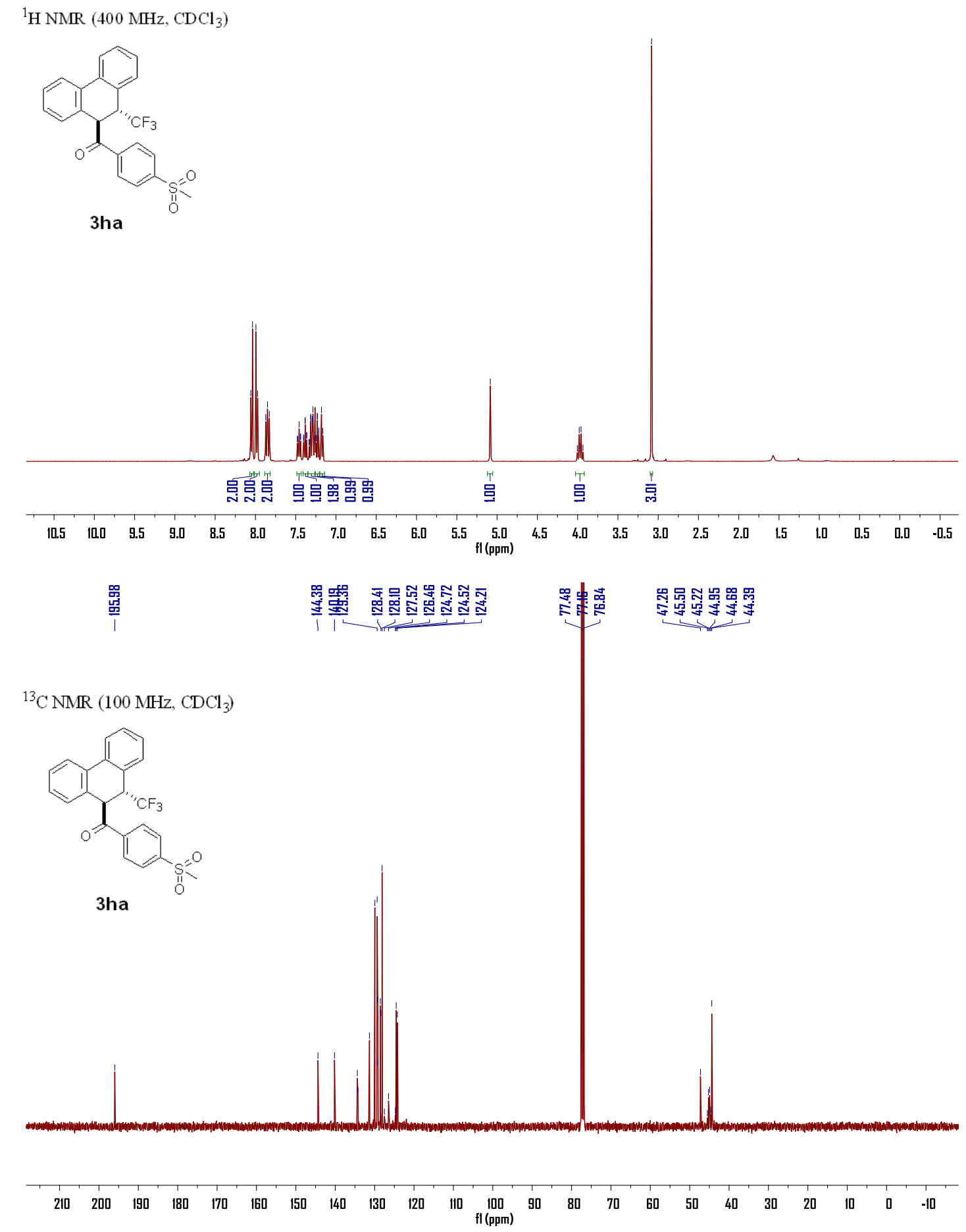
$\left.{ }^{19} \mathrm{~F} \mathrm{NMR} \mathrm{(376} \mathrm{MHz,} \mathrm{CDCl}_{3}\right)$

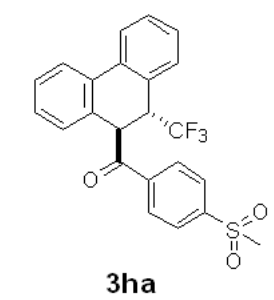

3ha

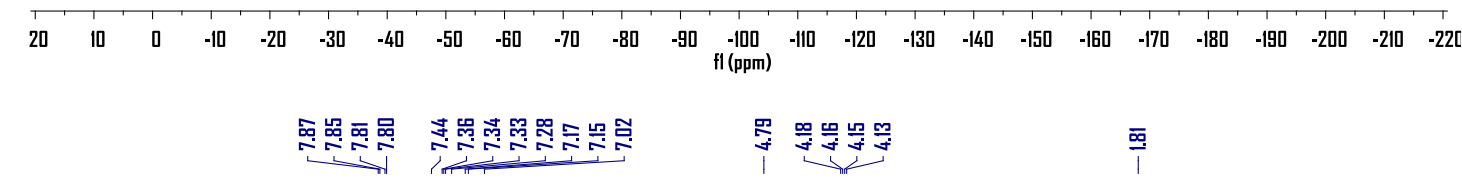

${ }^{1} \mathrm{H}$ NMR $\left(600 \mathrm{MHz}, \mathrm{CDCl}_{3}\right)$

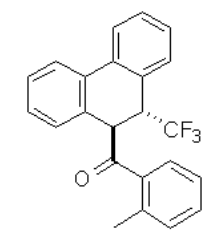

3ia

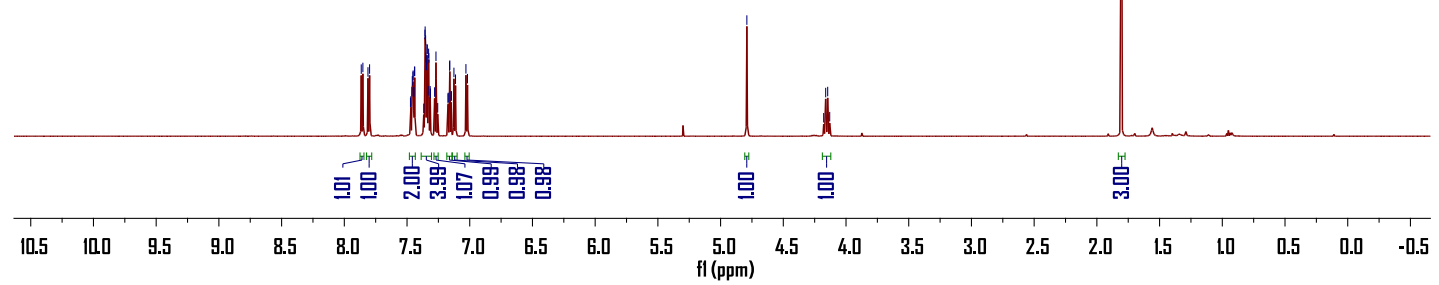




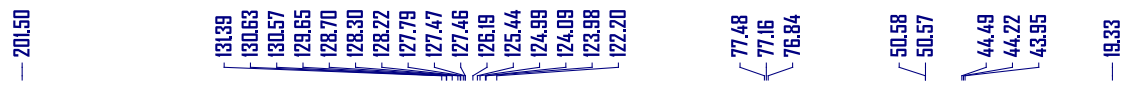

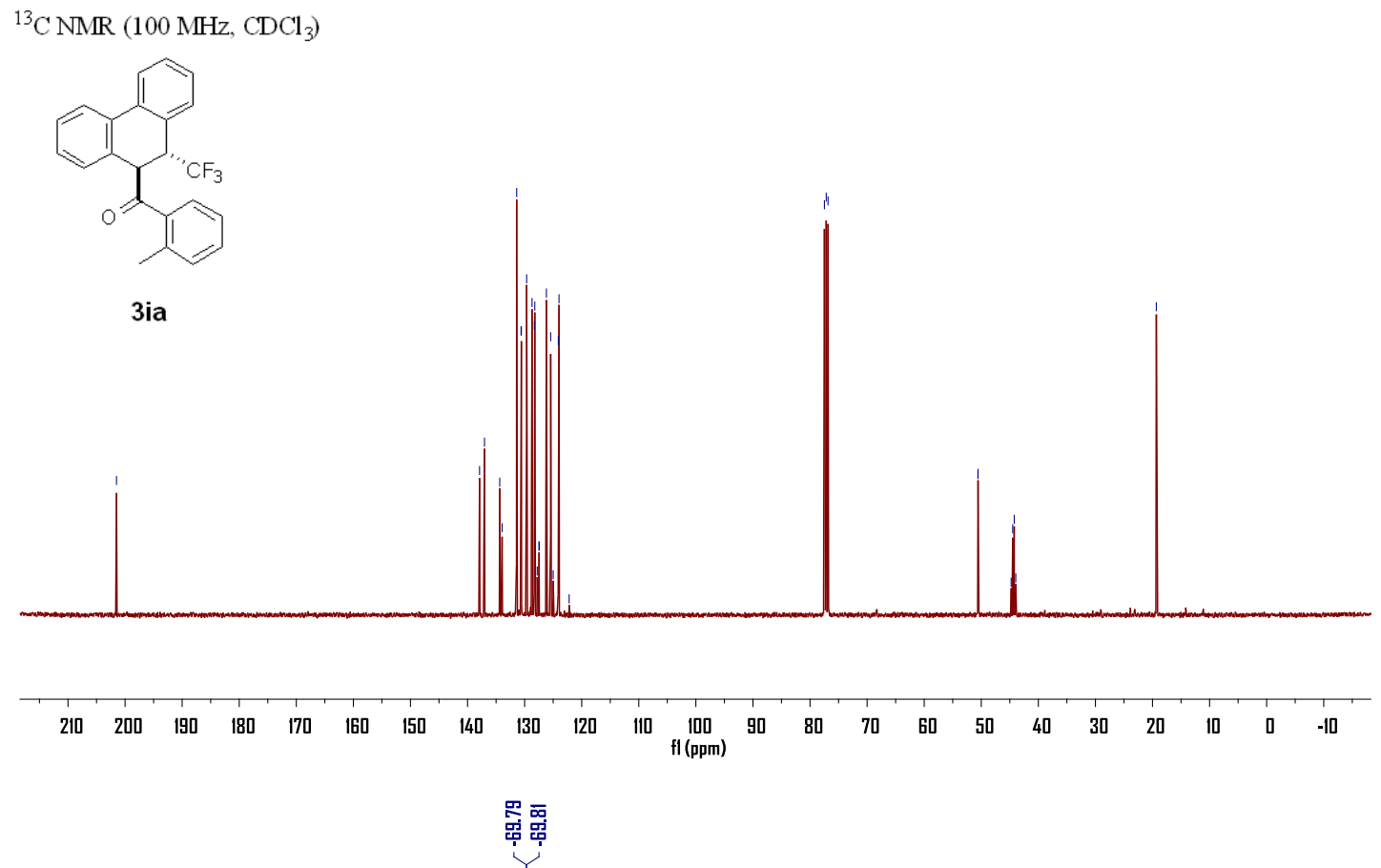

${ }^{19} \mathrm{FNMR}\left(376 \mathrm{MHz}, \mathrm{CDCl}_{3}\right)$

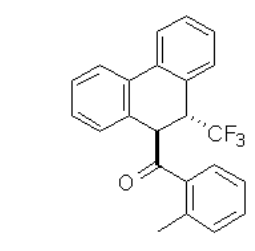

$3 i a$

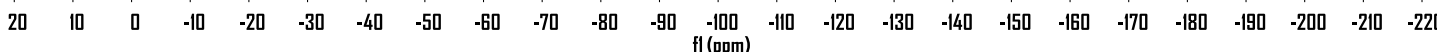




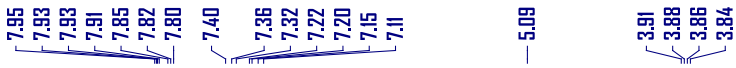

${ }^{1} \mathrm{H} \mathrm{NMR}\left(400 \mathrm{MHz}, \mathrm{CDCl}_{3}\right)$

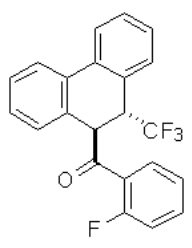

$3 \mathrm{ja}$

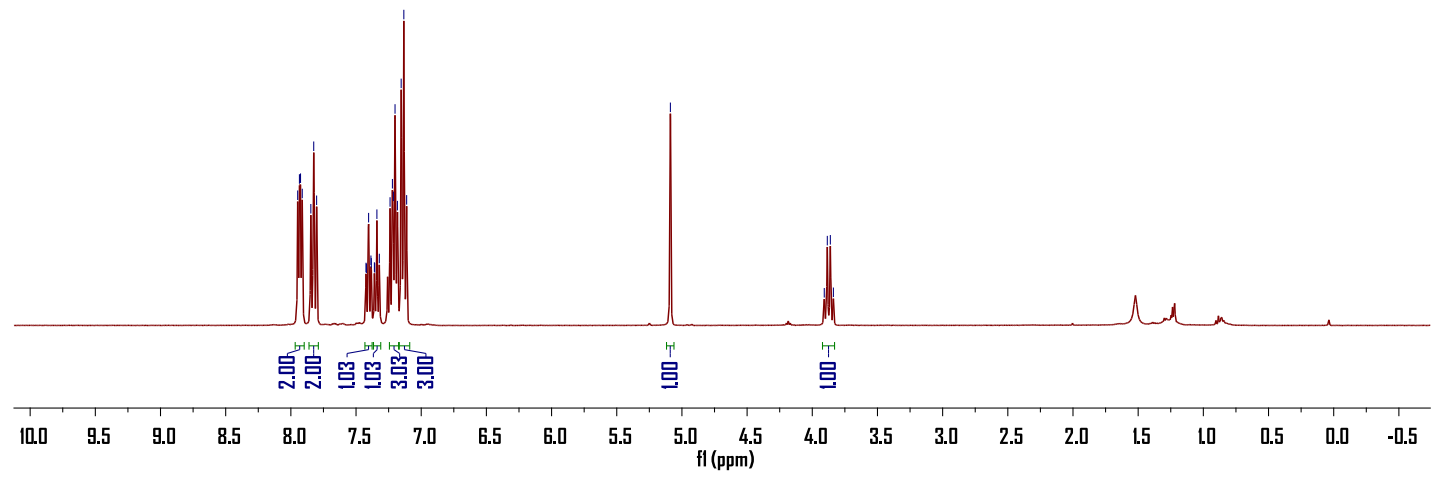

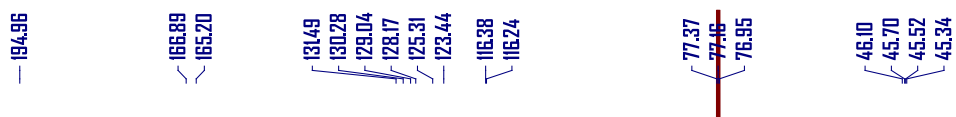

${ }^{13} \mathrm{C} \mathrm{NMR}\left(150 \mathrm{MHz}, \mathrm{CDCl}_{3}\right)$

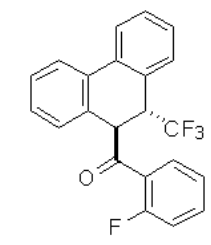

3ja

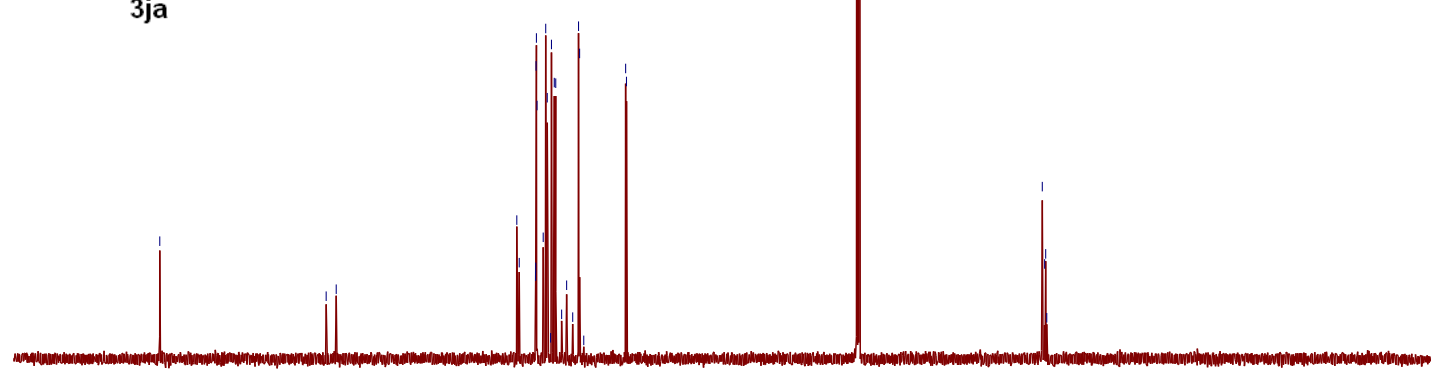

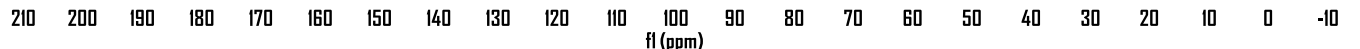




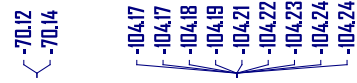

${ }^{19} \mathrm{~F} \mathrm{NMR}\left(376 \mathrm{MHz}, \mathrm{CDCl}_{3}\right)$

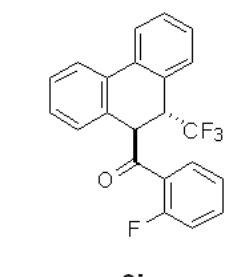

$3 \mathbf{j a}$

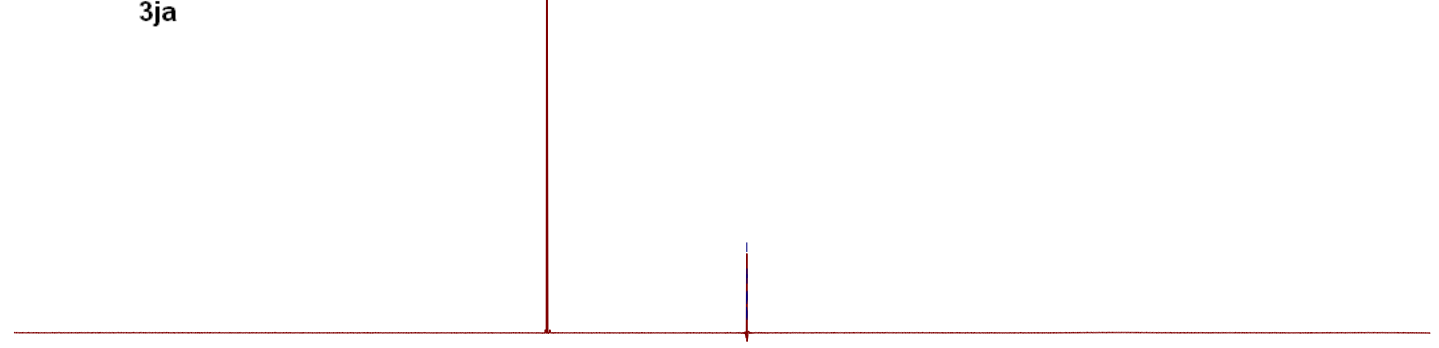

\begin{tabular}{lllllllllllllllllllllllllllllll}
\hline 20 & 10 & 0 & -10 & -20 & -30 & -40 & -50 & -60 & -70 & -80 & -90 & -100 & -110 & -120 & -130 & -140 & -150 & -160 & -170 & -180 & -190 & -200 & -210 & -220
\end{tabular}

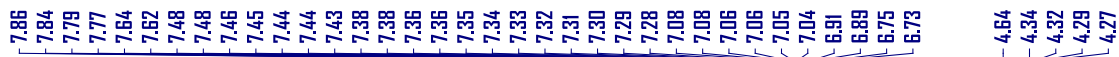

${ }^{1} \mathrm{HNMR}\left(400 \mathrm{MHz}, \mathrm{CDCl}_{3}\right)$

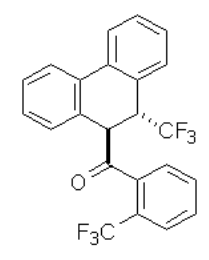

3ka

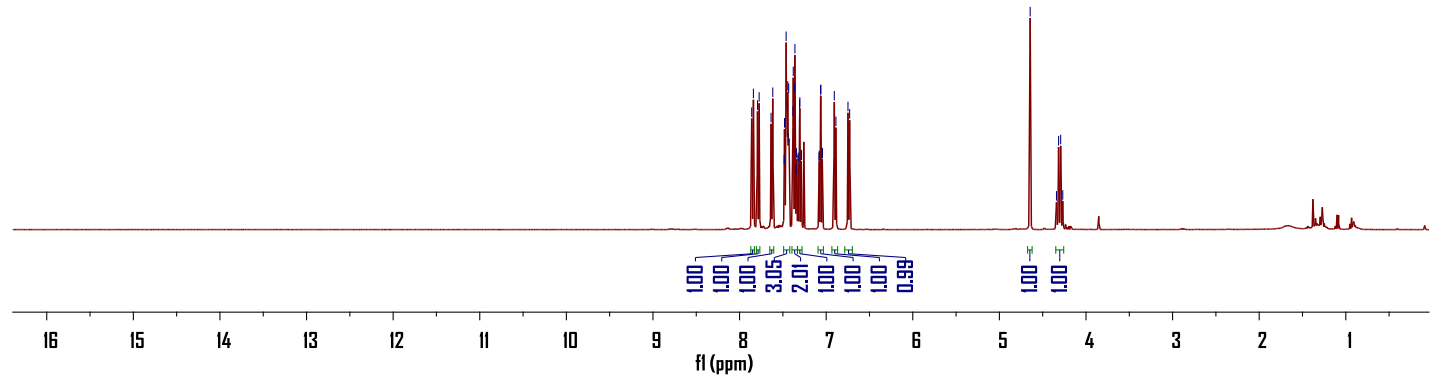




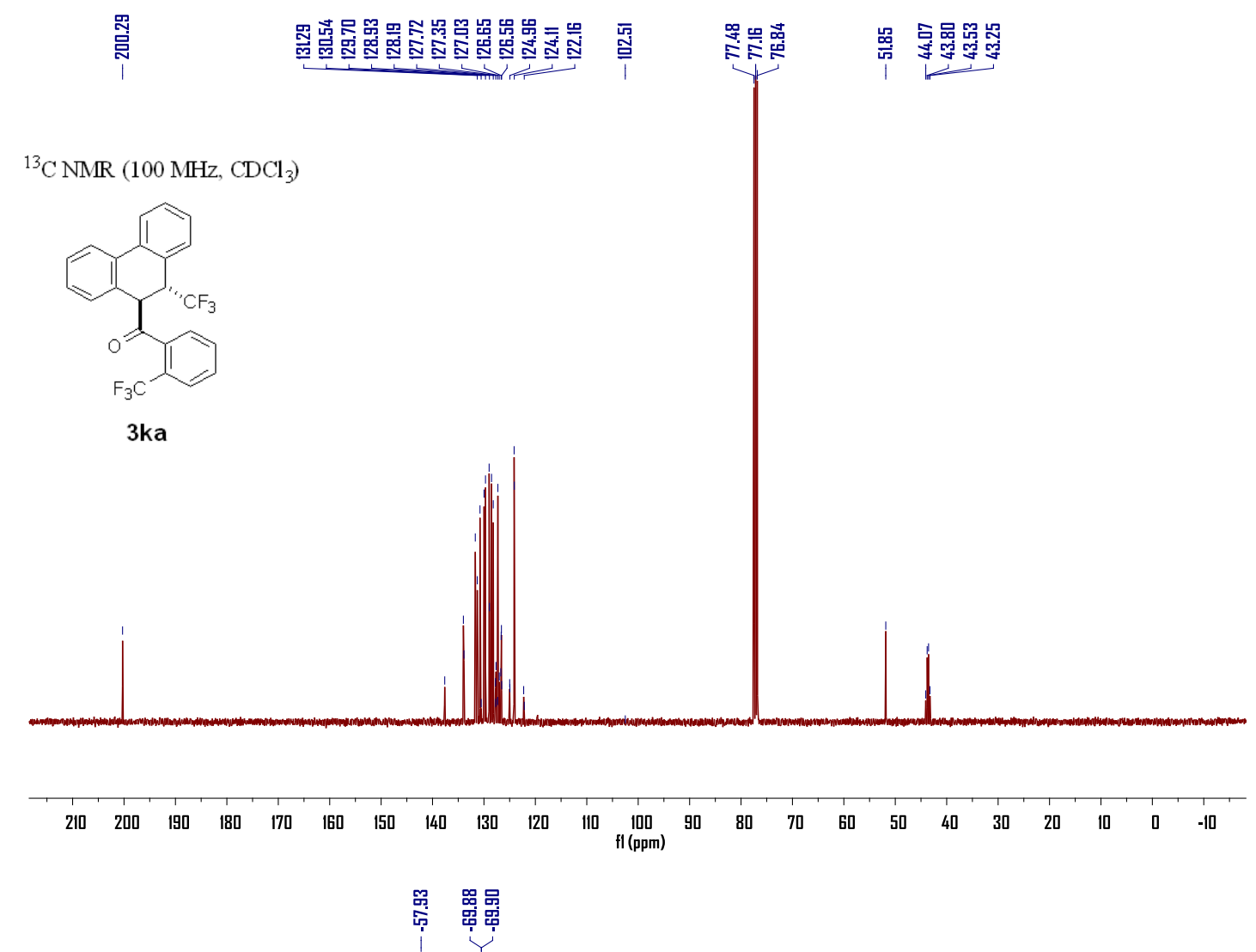

${ }^{19} \mathrm{~F} \mathrm{NMR}\left(376 \mathrm{MHz}, \mathrm{CDCl}_{3}\right)$

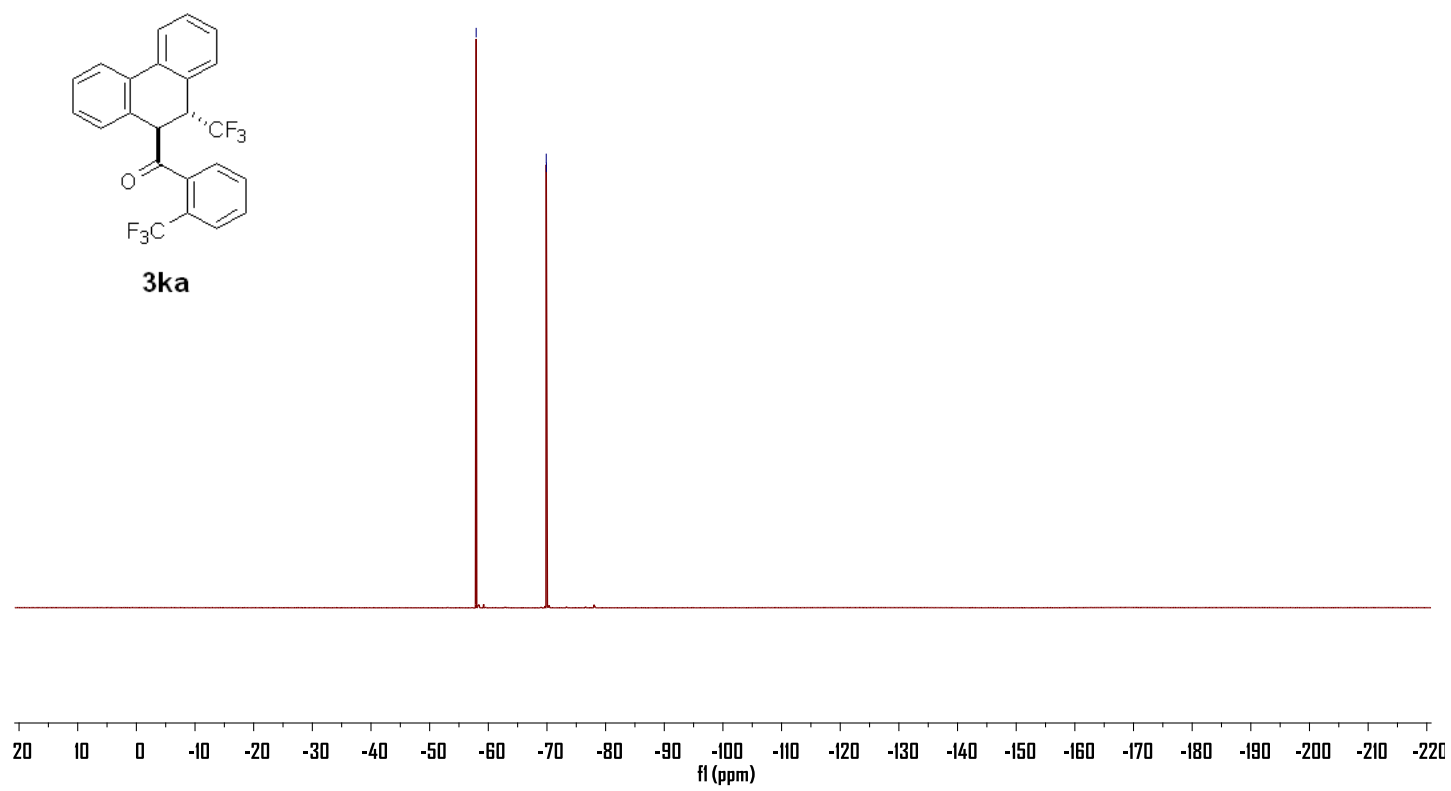




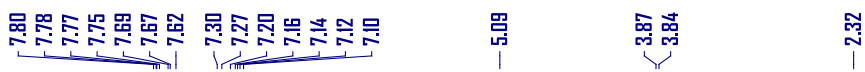

${ }^{1} \mathrm{H} \mathrm{NMR}\left(400 \mathrm{MHz}, \mathrm{CDCl}_{3}\right)$

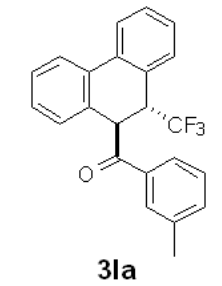

3la

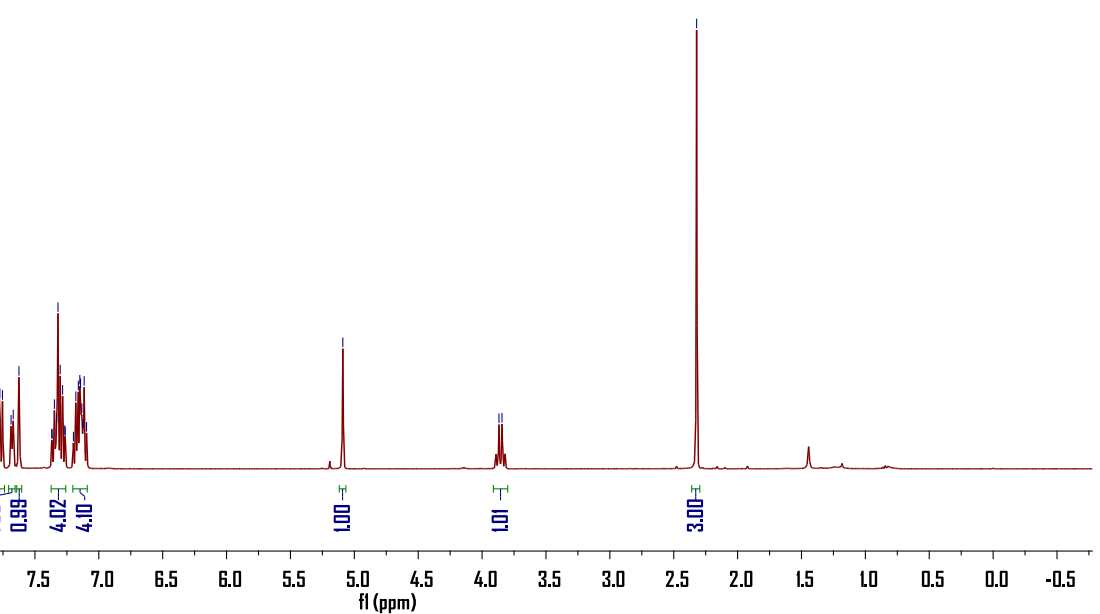

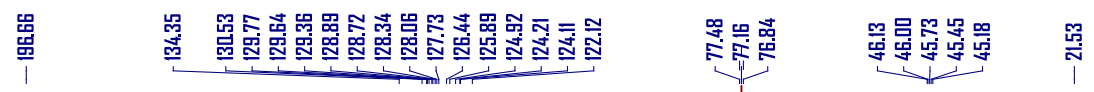

${ }^{13} \mathrm{C} \mathrm{NMR}\left(100 \mathrm{MHz}, \mathrm{CDCl}_{3}\right)$

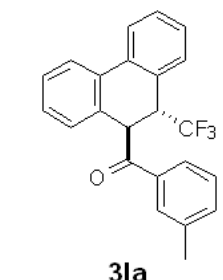

$31 a$

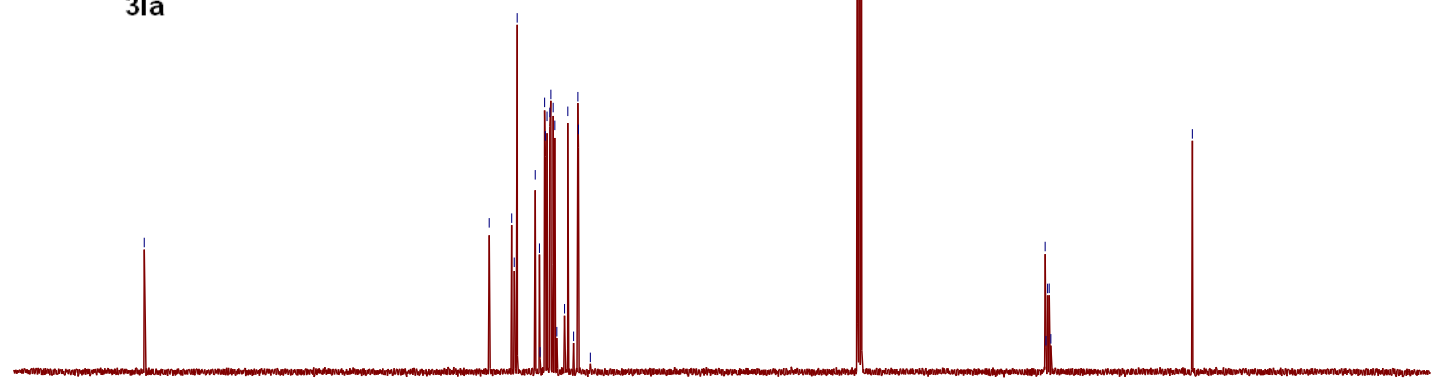

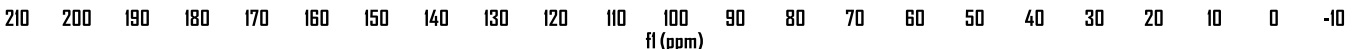




\section{올 뭄}

${ }^{19} \mathrm{~F} \mathrm{NMR}(376 \mathrm{MHz}, \mathrm{C}$

$\begin{array}{lllllllllllllllllllllllllllllll}20 & 10 & 0 & -10 & -20 & -30 & -40 & -50 & -60 & -70 & -80 & -90 & -100 & -110 & -120 & -130 & -140 & -150 & -160 & -170 & -180 & -190 & -200 & -210 & -220\end{array}$

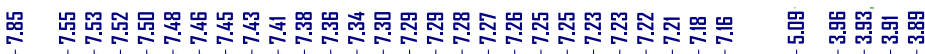

${ }^{1} \mathrm{H} \mathrm{NMR}\left(400 \mathrm{MHz}, \mathrm{CDCl}_{3}\right)$

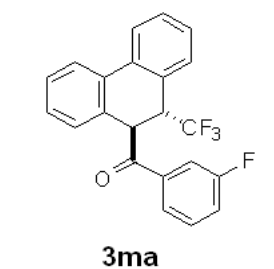

3ma

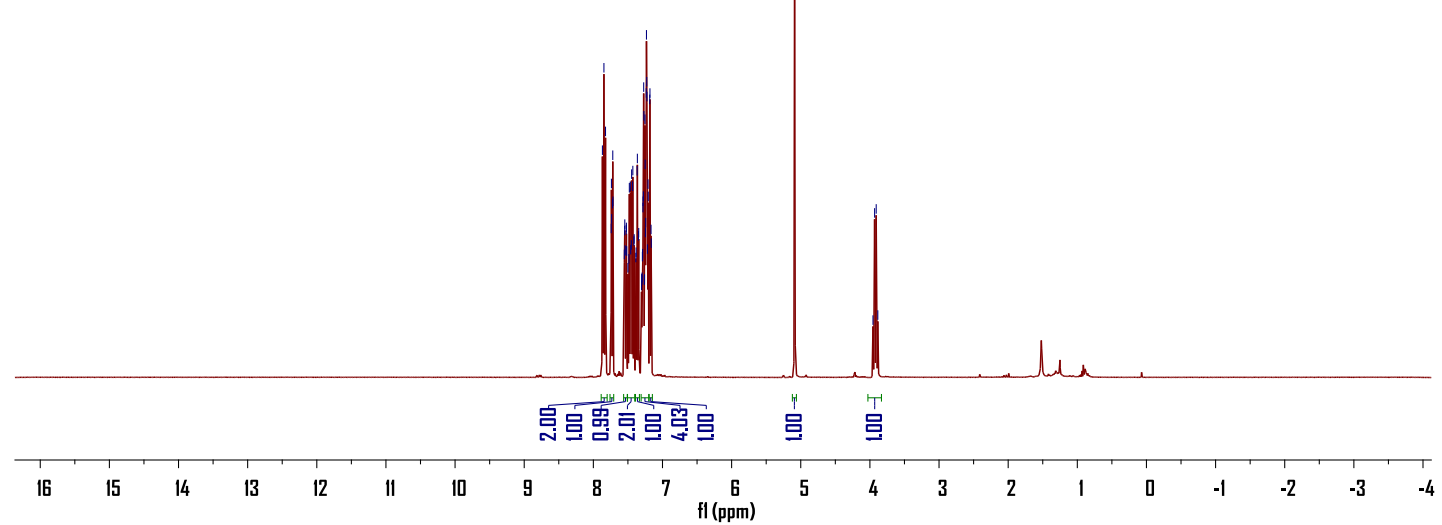




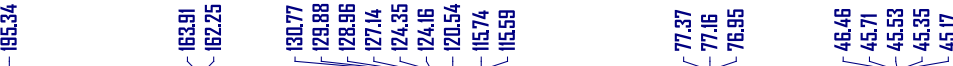

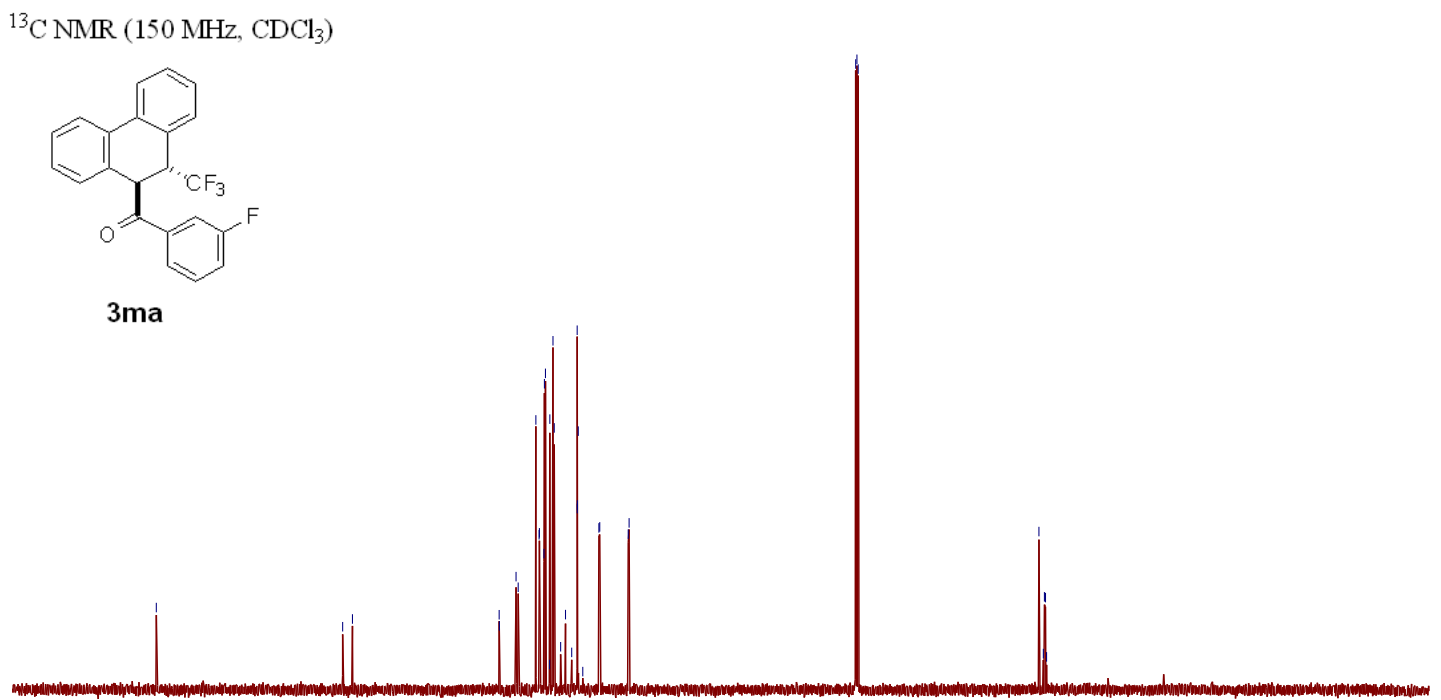

$\begin{array}{lllllllllllllllllllllll}210 & 200 & 190 & 180 & 170 & 160 & 150 & 140 & 130 & 120 & 110 & \begin{array}{ll}100 \\ \mathrm{fl}(\mathrm{ppm})\end{array} & 90 & 80 & 70 & 60 & 50 & 40 & 30 & 20 & 10 & 0 & -10\end{array}$

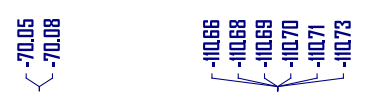

${ }^{19} \mathrm{~F} \mathrm{NMR}\left(376 \mathrm{MHz}, \mathrm{CDCl}_{3}\right)$

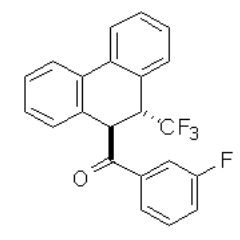

$3 \mathrm{ma}$

$\begin{array}{lllllllllllllllllllllllllllll}20 & 10 & 0 & -10 & -20 & -30 & -40 & -50 & -60 & -70 & -80 & -90 & -100 & -110 & -120 & -130 & -140 & -150 & -160 & -170 & -180 & -190 & -200 & -210 & -220\end{array}$ 


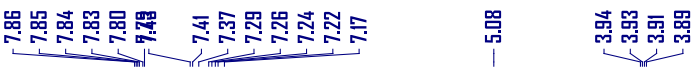

${ }^{1} \mathrm{H} \mathrm{NMR}\left(600 \mathrm{MHz}, \mathrm{CDCl}_{3}\right)$

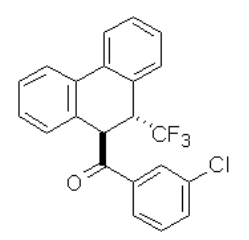

3na

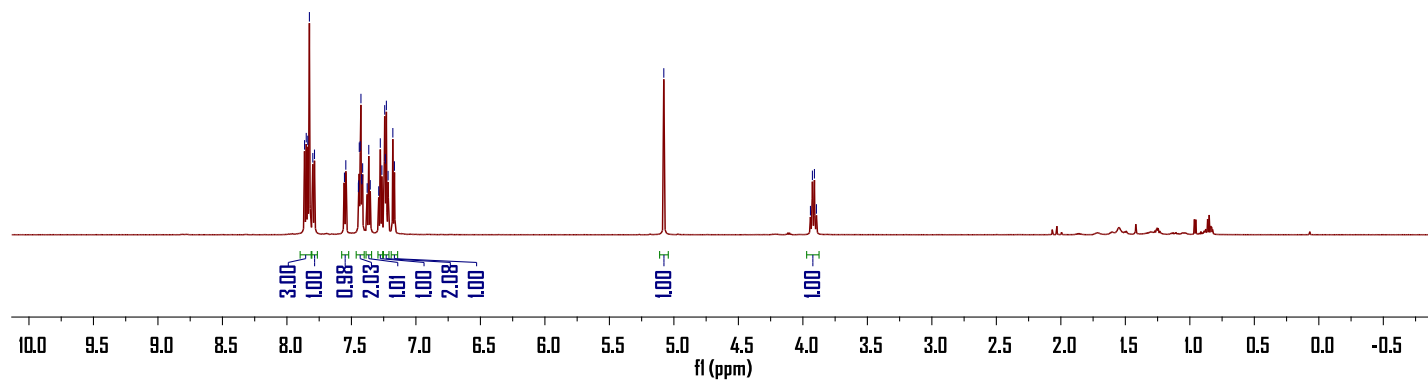

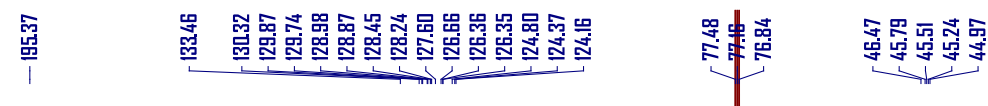

${ }^{13} \mathrm{C} \mathrm{NMR}\left(100 \mathrm{MHz}, \mathrm{CDCl}_{3}\right)$

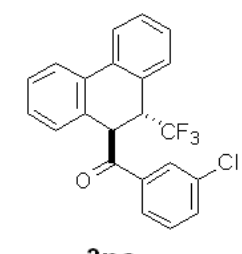

3na

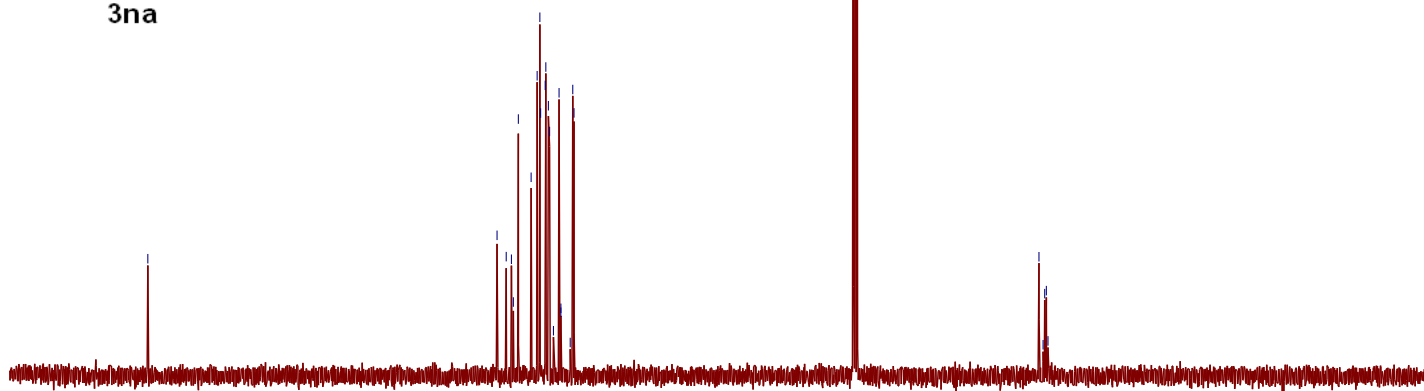

$\begin{array}{lllllllllllllllllllllll}210 & 200 & 190 & 180 & 170 & 160 & 150 & 140 & 130 & 120 & 110 & \underset{f l}{100} & 90 & 80 & 70 & 60 & 50 & 40 & 30 & 20 & 10 & 0 & -10\end{array}$ 


\section{总亮}

${ }^{19} \mathrm{~F} \mathrm{NMR}\left(376 \mathrm{MHz}, \mathrm{CDCl}_{3}\right)$

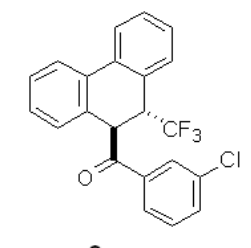

3na

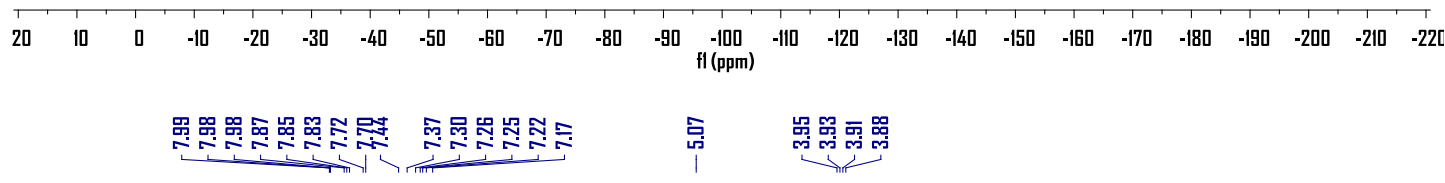

${ }^{1} \mathrm{H}$ NMR $\left(400 \mathrm{MHz}, \mathrm{CDCl}_{3}\right)$

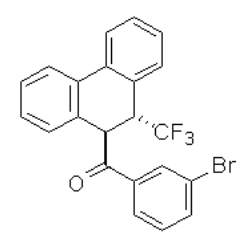

$30 a$

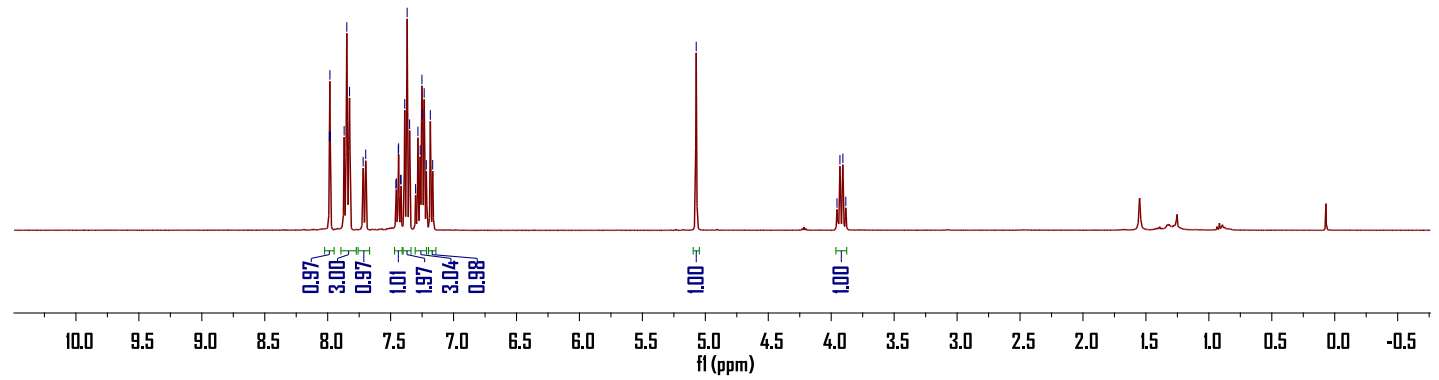




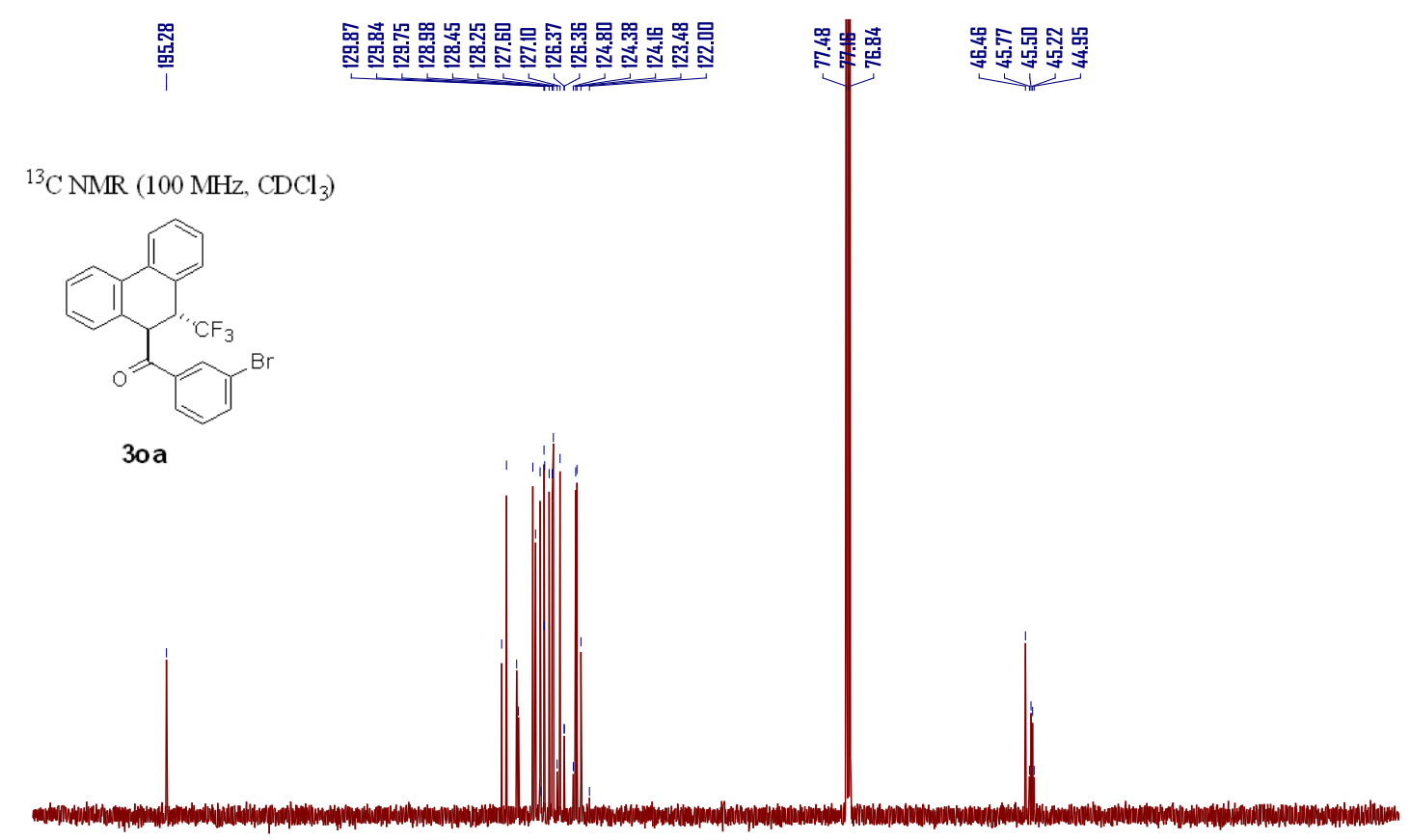

$\begin{array}{llllllllllllllllllllllllll}210 & 200 & 190 & 180 & 170 & 160 & 150 & 140 & 130 & 120 & 110 & \begin{array}{c}100 \\ \mathrm{fl}(\mathrm{ppm})\end{array} & 90 & 80 & 70 & 60 & 50 & 40 & 30 & 20 & 10 & 0 & -10\end{array}$

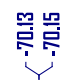

${ }^{19} \mathrm{~F} \mathrm{NMR}\left(376 \mathrm{MHz}, \mathrm{CDCl}_{3}\right)$

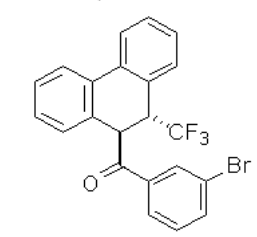

3oa

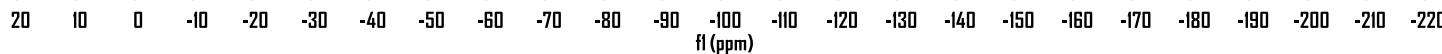


${ }^{1} \mathrm{H} \mathrm{NMR}\left(600 \mathrm{MHz}, \mathrm{CDCl}_{3}\right)$<smiles>O=C(c1cccc(C(F)(F)F)c1)C1c2ccccc2-c2ccccc2C1C(F)(F)F</smiles>

3 pa

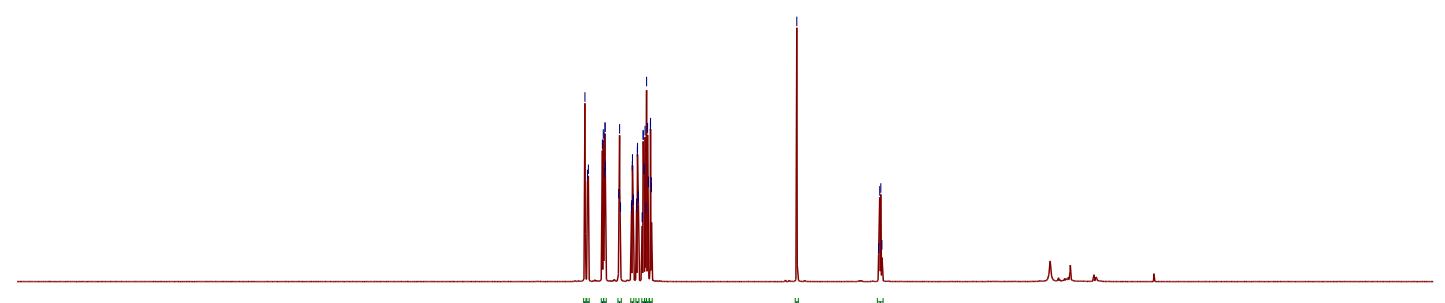

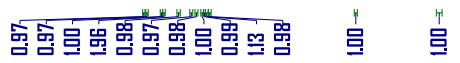

$\begin{array}{llllllllllllllllllllllll}16 & 15 & 14 & 13 & 12 & 11 & 10 & 9 & 8 & 7 & \underset{f l}{f(p p m)} & 6 & 4 & 3 & 2 & 1 & 0 & -1 & -2 & -3\end{array}$

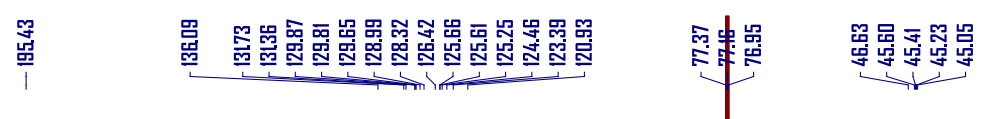

${ }^{13} \mathrm{C} \mathrm{NMR}\left(150 \mathrm{MHz}, \mathrm{CDCl}_{3}\right)$

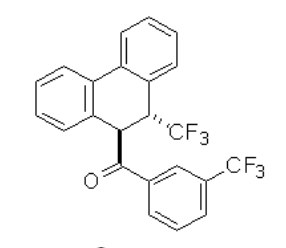

3 a

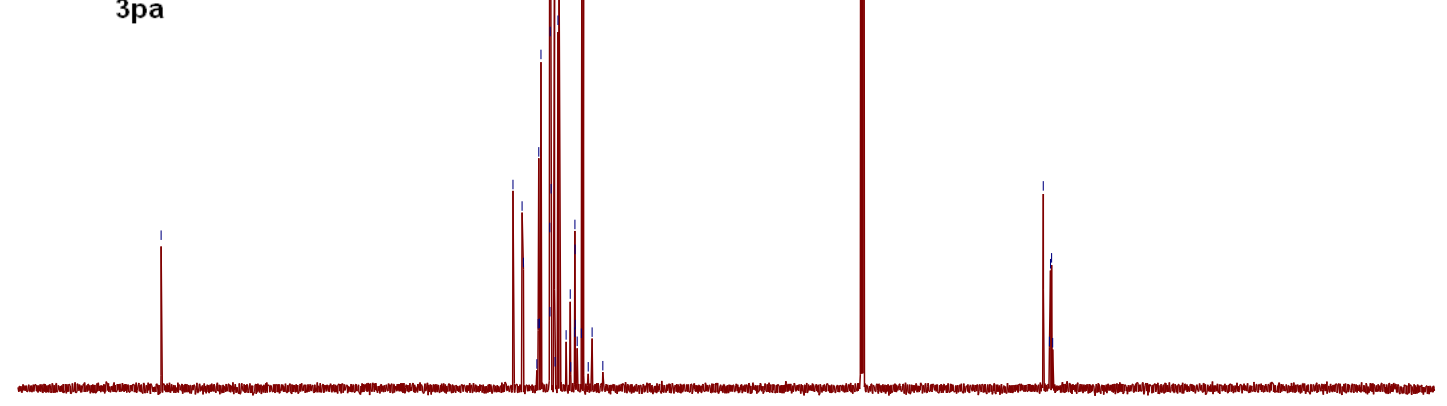

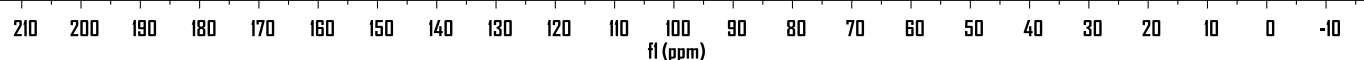




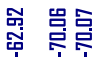

${ }^{19} \mathrm{~F} \mathrm{NMR}\left(565 \mathrm{MHz}, \mathrm{CDCl}_{3}\right)$

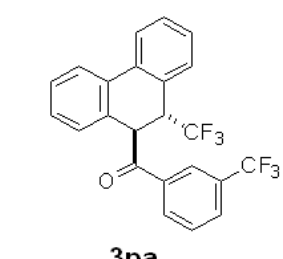

3 pa

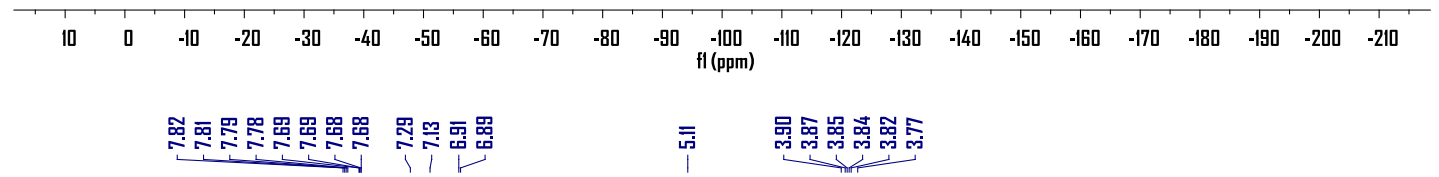

${ }^{1} \mathrm{H}$ NMR $\left(600 \mathrm{MHz}, \mathrm{CDCl}_{3}\right)$

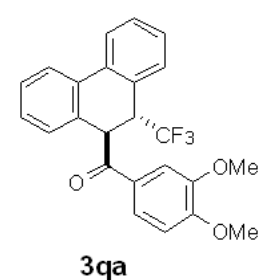

3qa

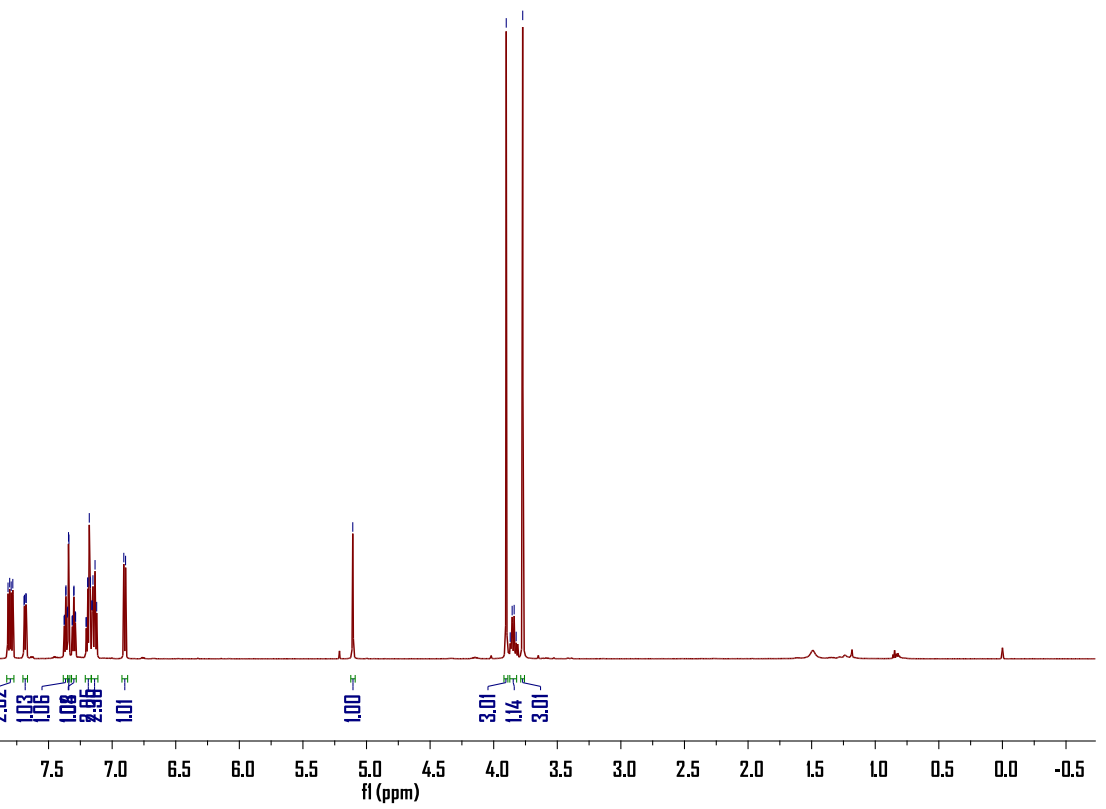




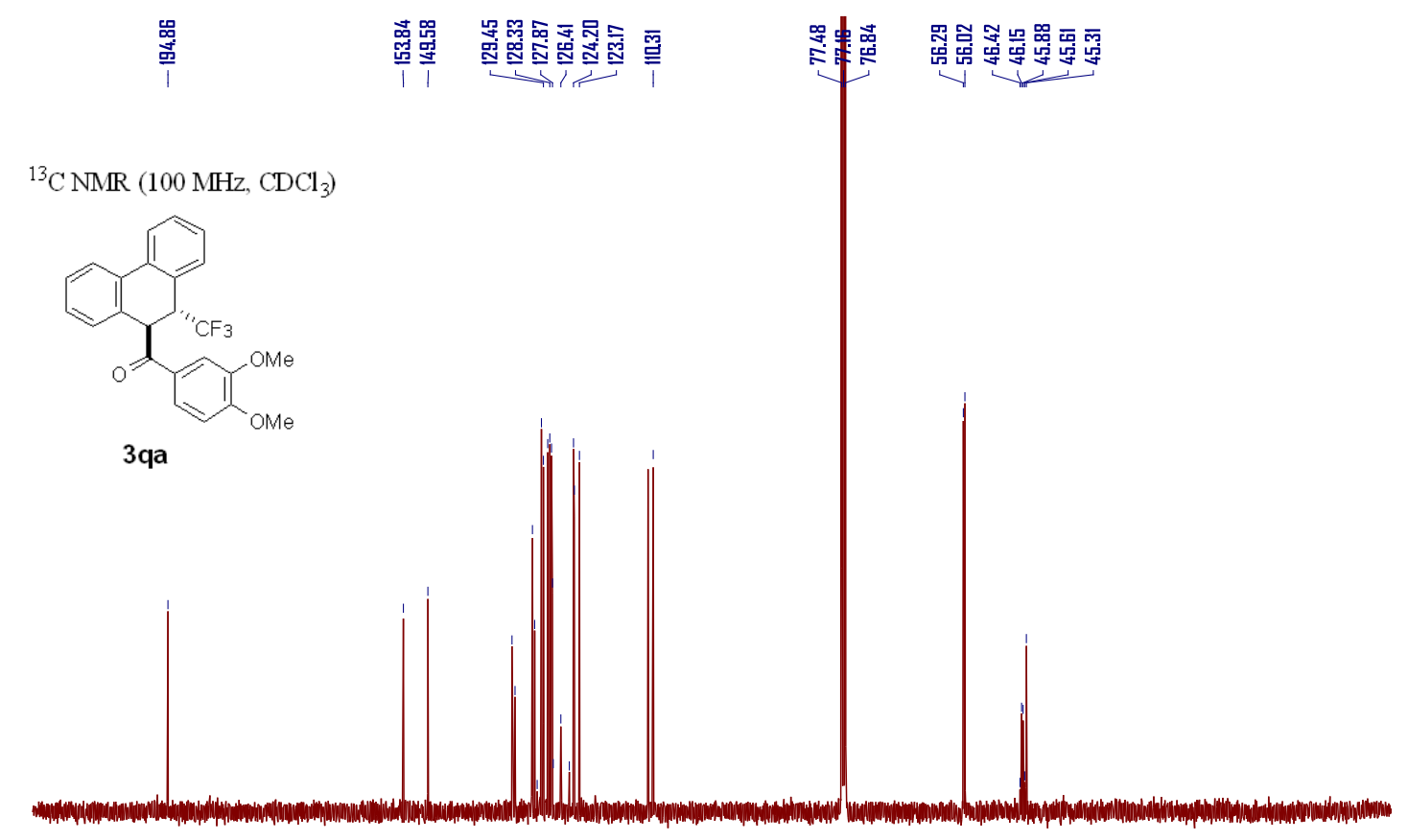

$\begin{array}{llllllllllllllllllllllllll}210 & 200 & 190 & 180 & 170 & 160 & 150 & 140 & 130 & 120 & 110 & \begin{array}{c}100 \\ \mathrm{fl}(\mathrm{ppm})\end{array} & 90 & 80 & 70 & 60 & 50 & 40 & 30 & 20 & 10 & 0 & -10\end{array}$

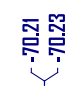

$\left.{ }^{19} \mathrm{~F} \mathrm{NMR} \mathrm{(376} \mathrm{MHz,} \mathrm{CDCl}_{3}\right)$
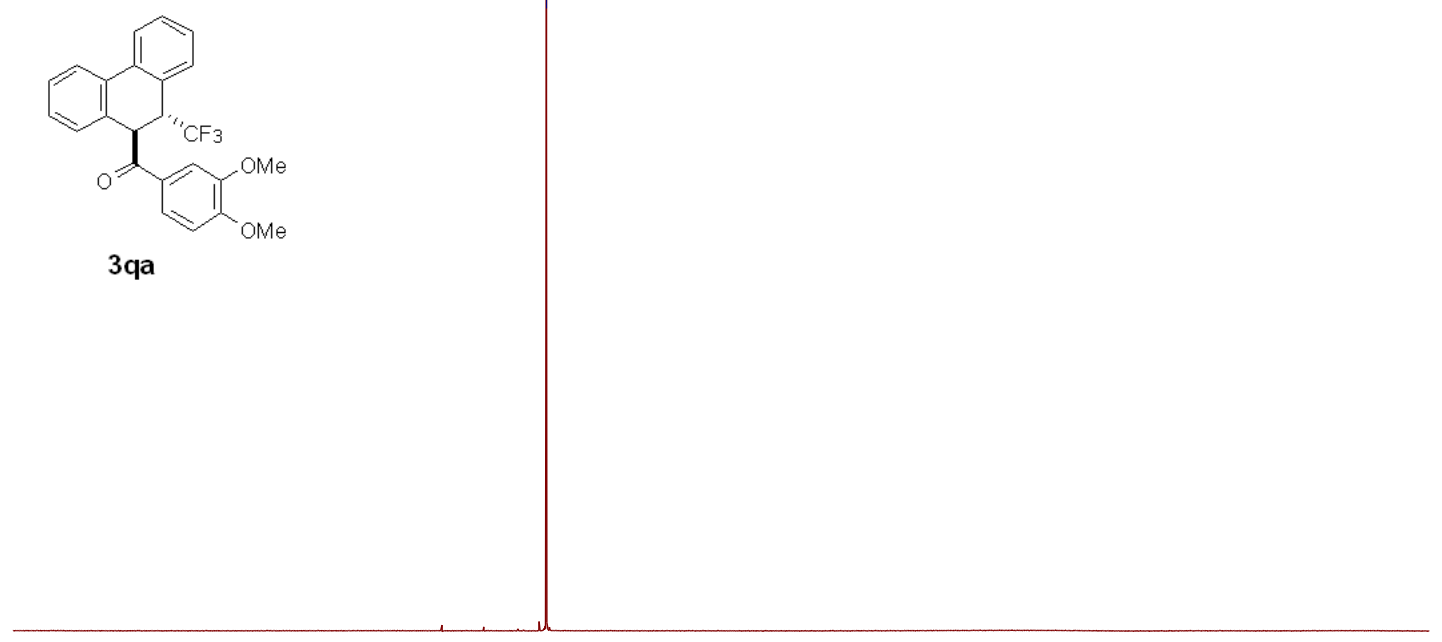

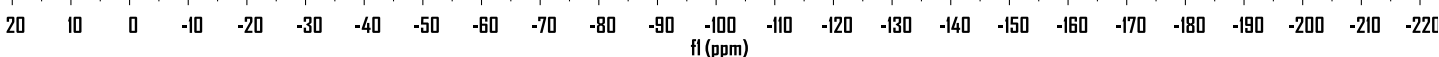




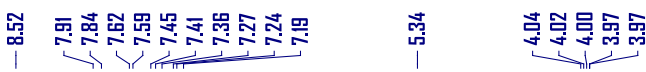

${ }^{1} \mathrm{H} \mathrm{NMR}\left(400 \mathrm{MHz}, \mathrm{CDCl}_{3}\right)$

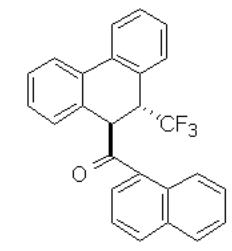

3ra

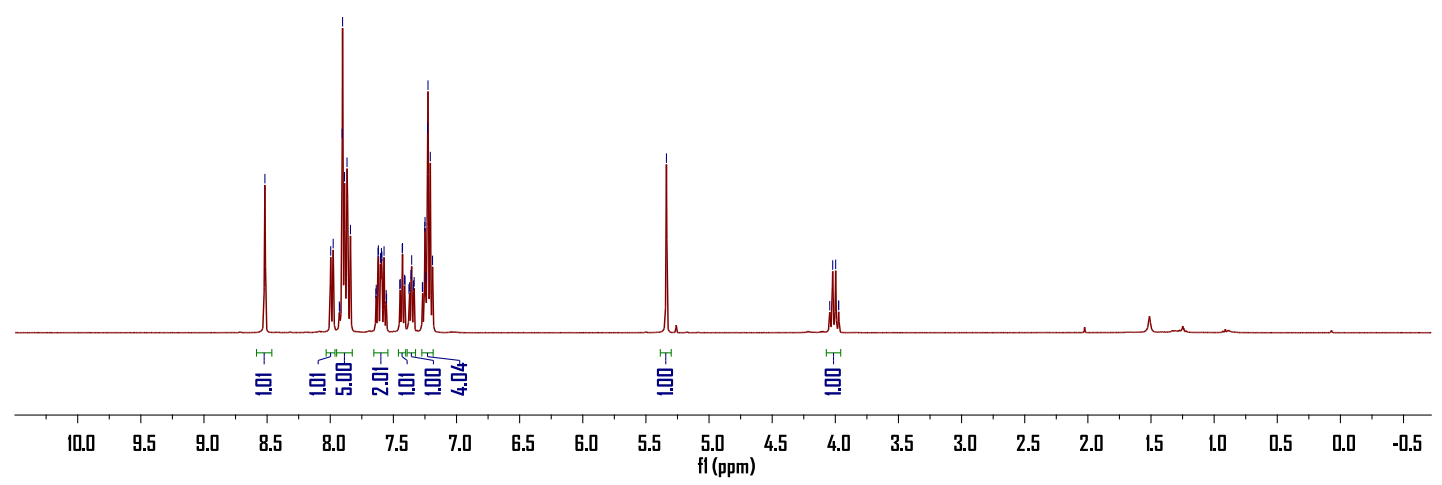

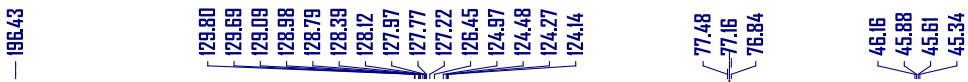

${ }^{13} \mathrm{C} \mathrm{NMR}\left(100 \mathrm{MHz}, \mathrm{CDCl}_{3}\right)$

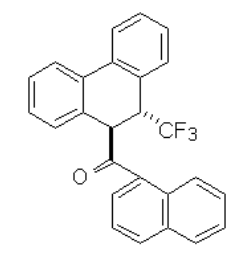

3ra

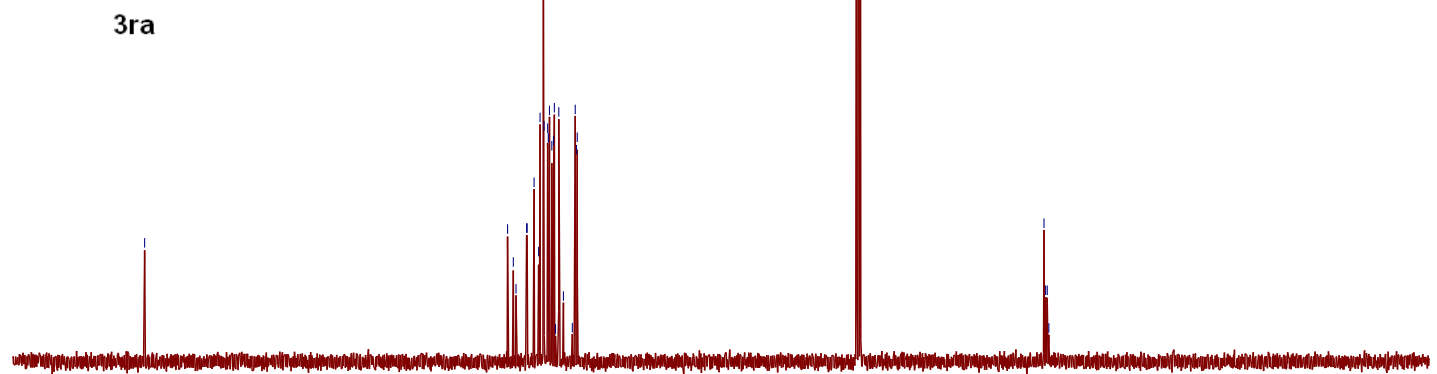

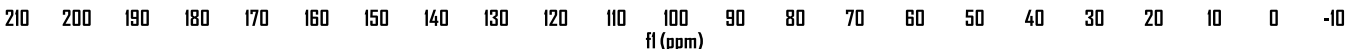


$\left.{ }^{19} \mathrm{~F} \mathrm{NMR} \mathrm{(376} \mathrm{MHz,} \mathrm{CDCl}_{3}\right)$

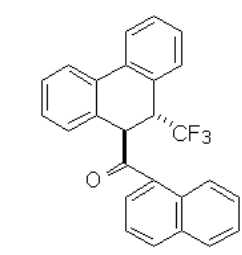

3ra

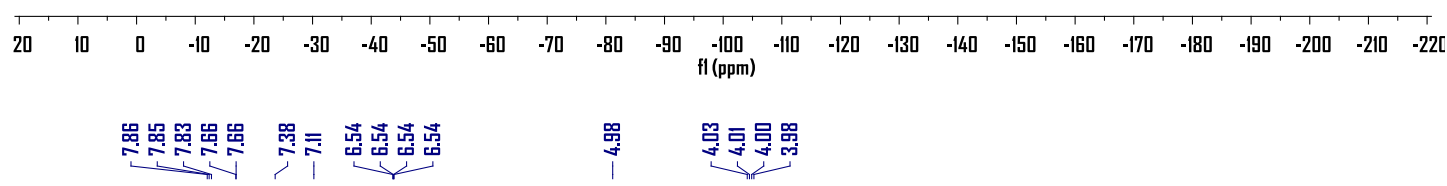

${ }^{1} \mathrm{H} \mathrm{NMR}\left(600 \mathrm{MHz}, \mathrm{CDCl}_{3}\right)$

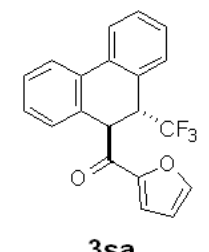

sa

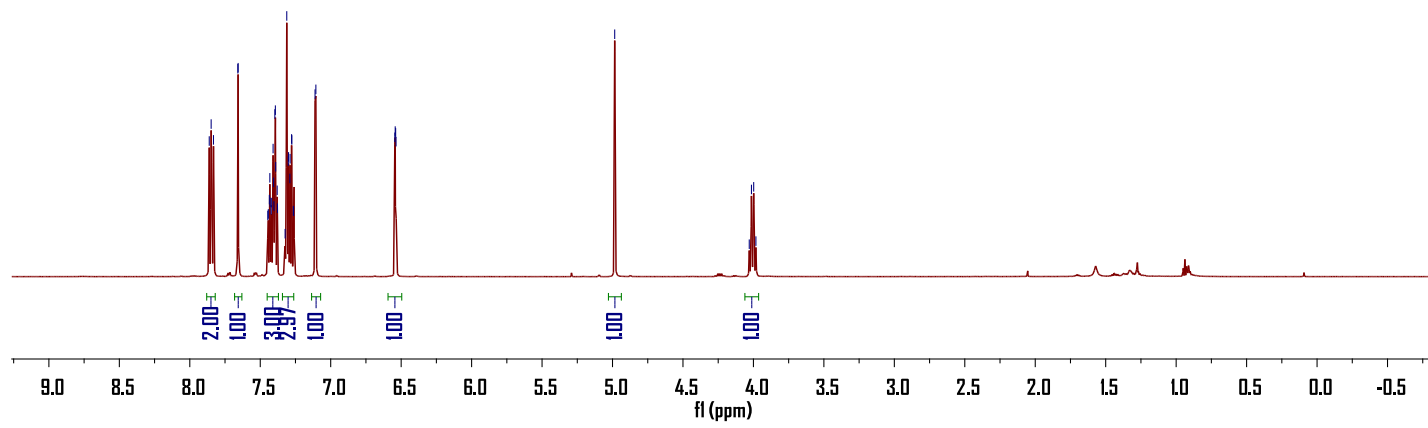




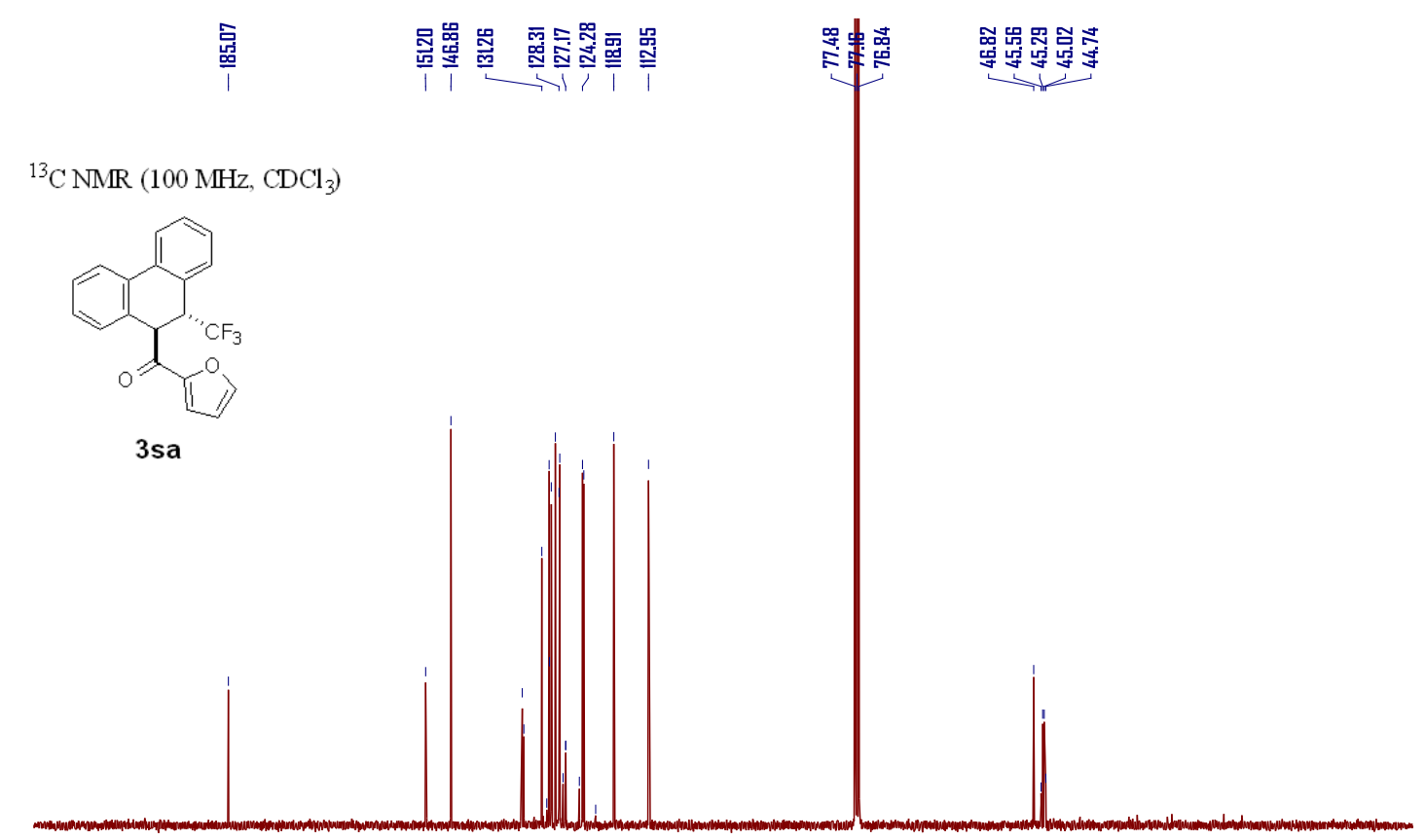

$\begin{array}{lllllllllllllllllllllllllllllll}210 & 200 & 190 & 180 & 170 & 160 & 150 & 140 & 130 & 120 & 110 & \begin{array}{c}100 \\ \mathrm{fl}(\mathrm{ppm})\end{array} & 90 & 80 & 70 & 60 & 50 & 40 & 30 & 20 & 10 & 0 & -10\end{array}$

을 물

${ }^{19} \mathrm{~F} \mathrm{NMR}\left(376 \mathrm{MHz}, \mathrm{CDCl}_{3}\right)$

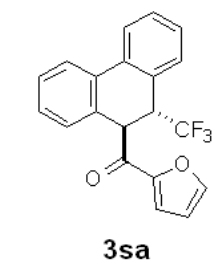

3 sa

$\begin{array}{lllllllllllllllllllllllllll}20 & 10 & 0 & -10 & -20 & -30 & -40 & -50 & -60 & -70 & -80 & -90 & -100 & -110 & -120 & -130 & -140 & -150 & -160 & -170 & -180 & -190 & -200 & -210 & -220\end{array}$ 
${ }^{1} \mathrm{HNMR}\left(400 \mathrm{MHz}, \mathrm{CDCl}_{3}\right)$<smiles>O=C1CCCc2c1c1ccccc1c1ccccc21</smiles>

3 ta

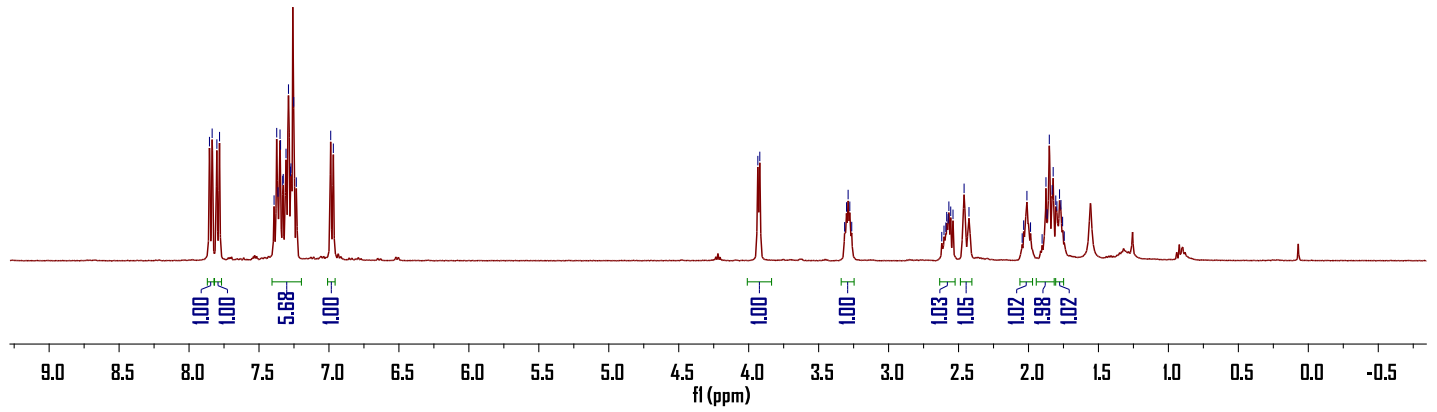

品

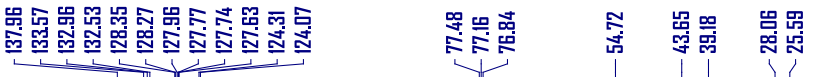

${ }^{13} \mathrm{C} \mathrm{NMR}\left(100 \mathrm{MHz}, \mathrm{CDCl}_{3}\right)$<smiles>O=C1CCCc2c1c1ccccc1c1ccccc21</smiles>

3ta

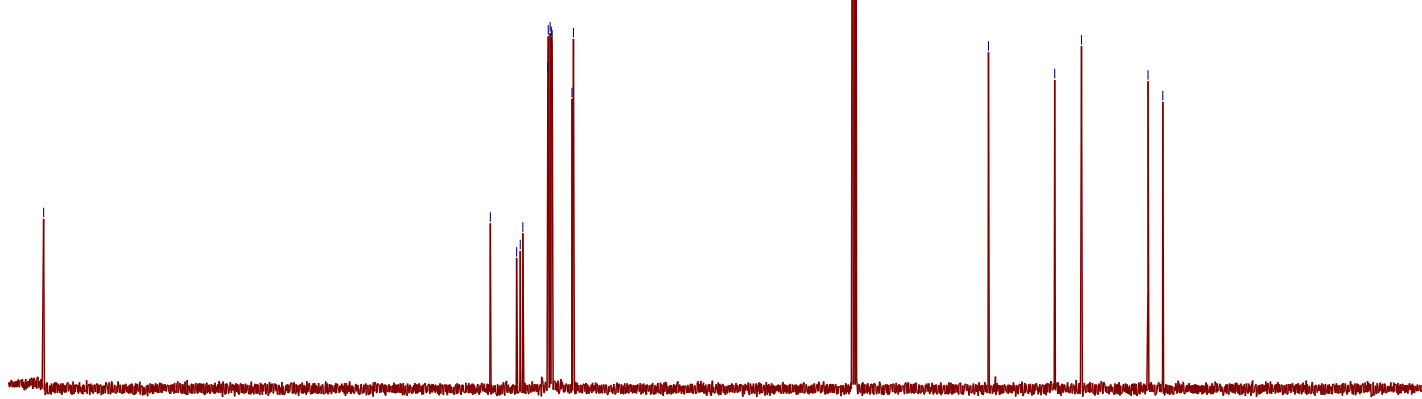

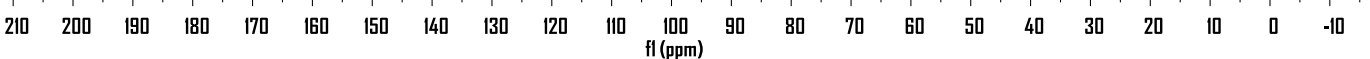


${ }^{1} \mathrm{H} \mathrm{NMR}\left(600 \mathrm{MHz}, \mathrm{CDCl}_{3}\right)$

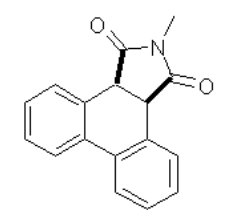

3ua
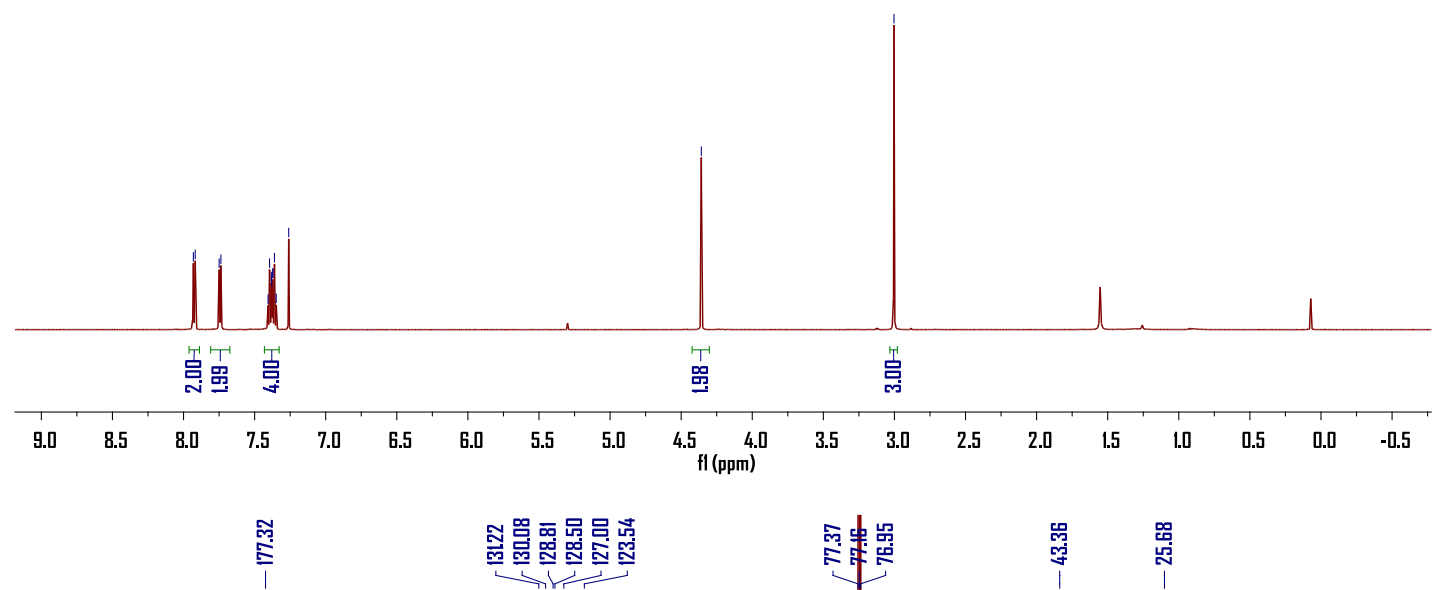

${ }^{13} \mathrm{C} \mathrm{NMR}\left(150 \mathrm{MHz}, \mathrm{CDCl}_{3}\right)$

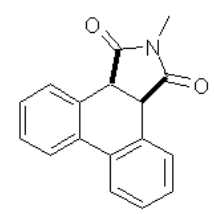

3ua

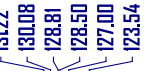

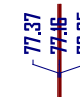

燢嵒

$\begin{array}{lllllllllllllllllllllll}210 & 200 & 190 & 180 & 170 & 160 & 150 & 140 & 130 & 120 & 110 & \begin{array}{c}100 \\ \mathrm{fl}(\mathrm{ppm})\end{array} & 90 & 80 & 70 & 60 & 50 & 40 & 30 & 20 & 10 & 0 & -10\end{array}$ 


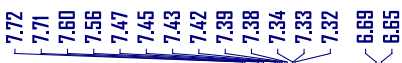

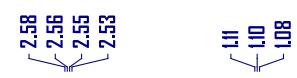

${ }^{1} \mathrm{H} \mathrm{NMR}\left(400 \mathrm{MHz}, \mathrm{CDCl}_{3}\right)$

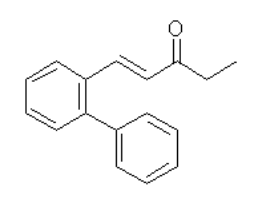

3va

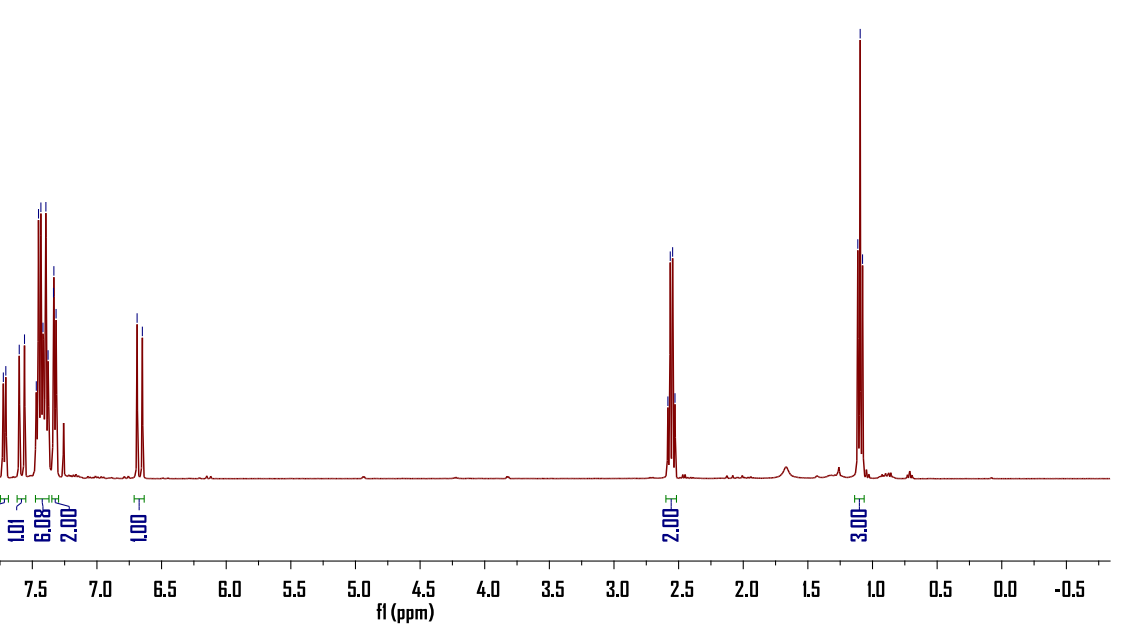

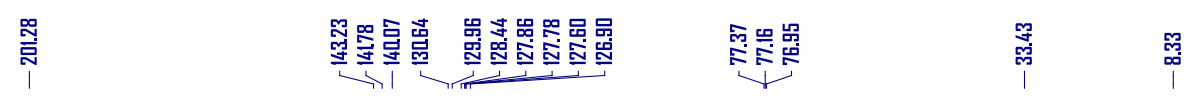

${ }^{13} \mathrm{C} \mathrm{NMR}\left(150 \mathrm{MHz}, \mathrm{CDCl}_{3}\right)$

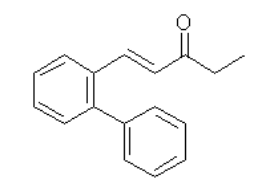

3va

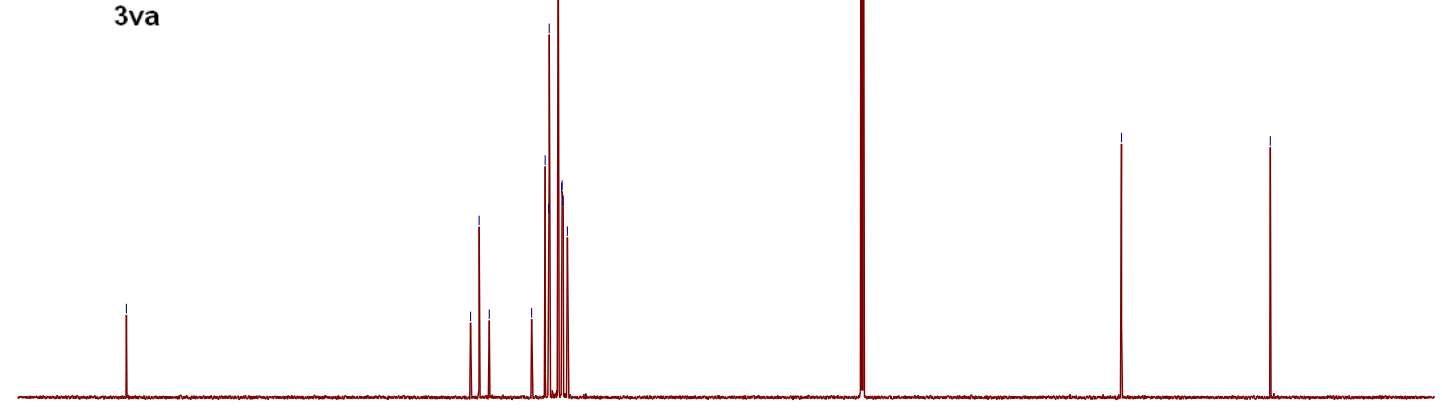

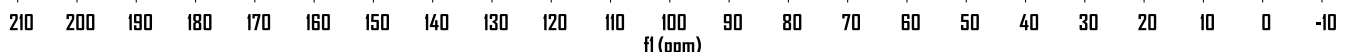


${ }^{1} \mathrm{H} \mathrm{NMR}\left(600 \mathrm{MHz}, \mathrm{CDCl}_{3}\right)$

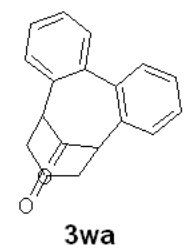

3wa

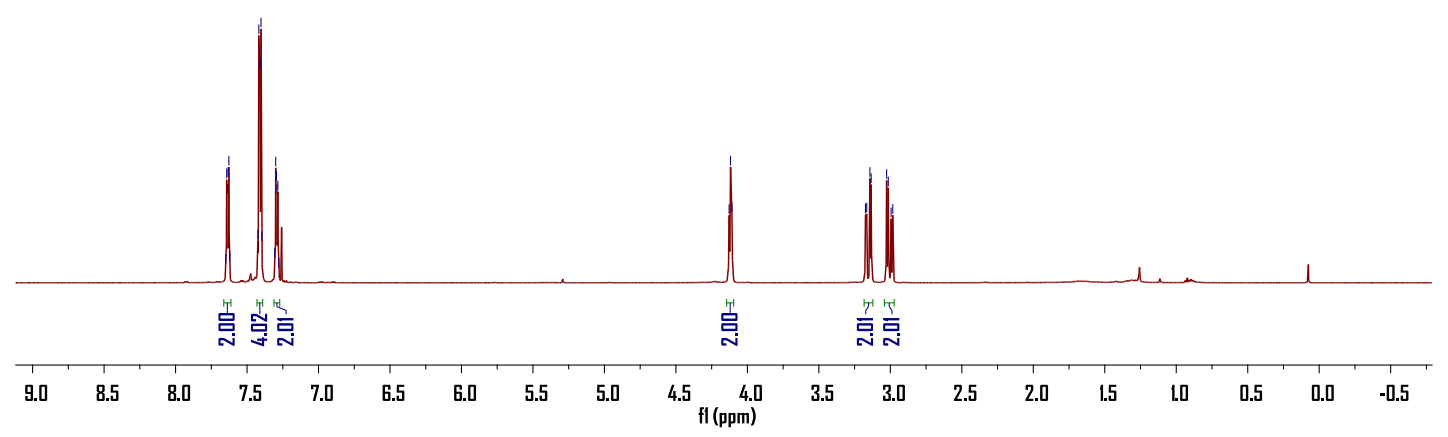

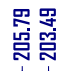

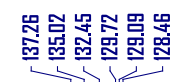

笑景品

聯鹗

${ }^{13} \mathrm{C} \mathrm{NMR}\left(150 \mathrm{MHz}, \mathrm{CDCl}_{3}\right)$

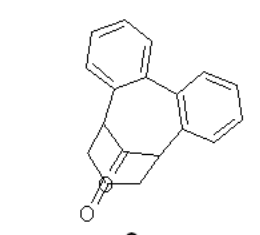

3wa

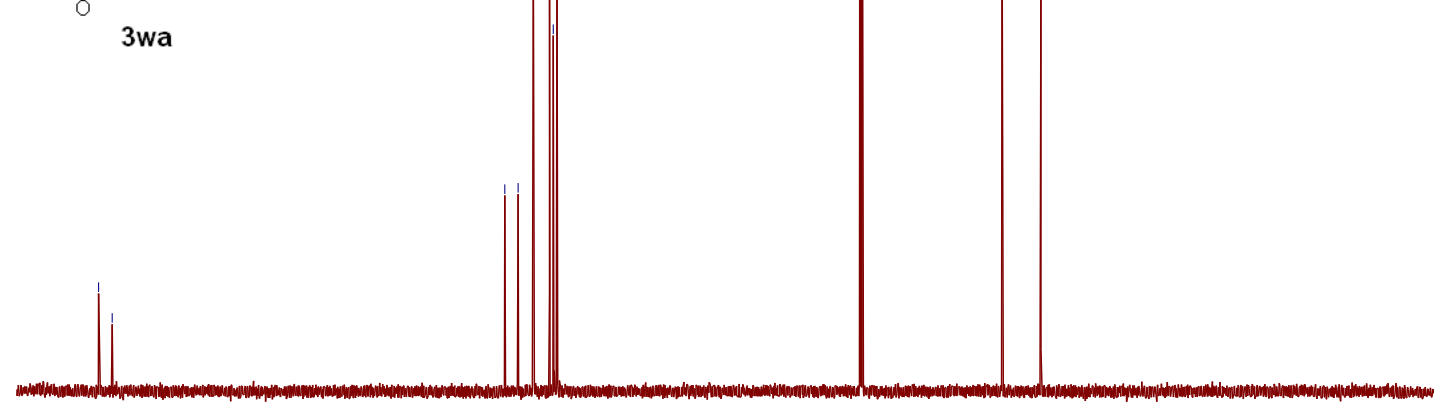

$\begin{array}{lllllllllllllllllllllll}210 & 200 & 190 & 180 & 170 & 160 & 150 & 140 & 130 & 120 & 110 & \begin{array}{c}100 \\ \mathrm{fl}(\mathrm{ppm})\end{array} & 90 & 80 & 70 & 60 & 50 & 40 & 30 & 20 & 10 & 0 & -10\end{array}$ 


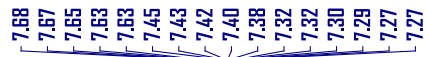

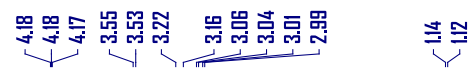

${ }^{1} \mathrm{H} \mathrm{NMR}\left(400 \mathrm{MHz}, \mathrm{CDCl}_{3}\right)$

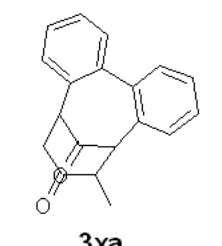

$3 \times a$

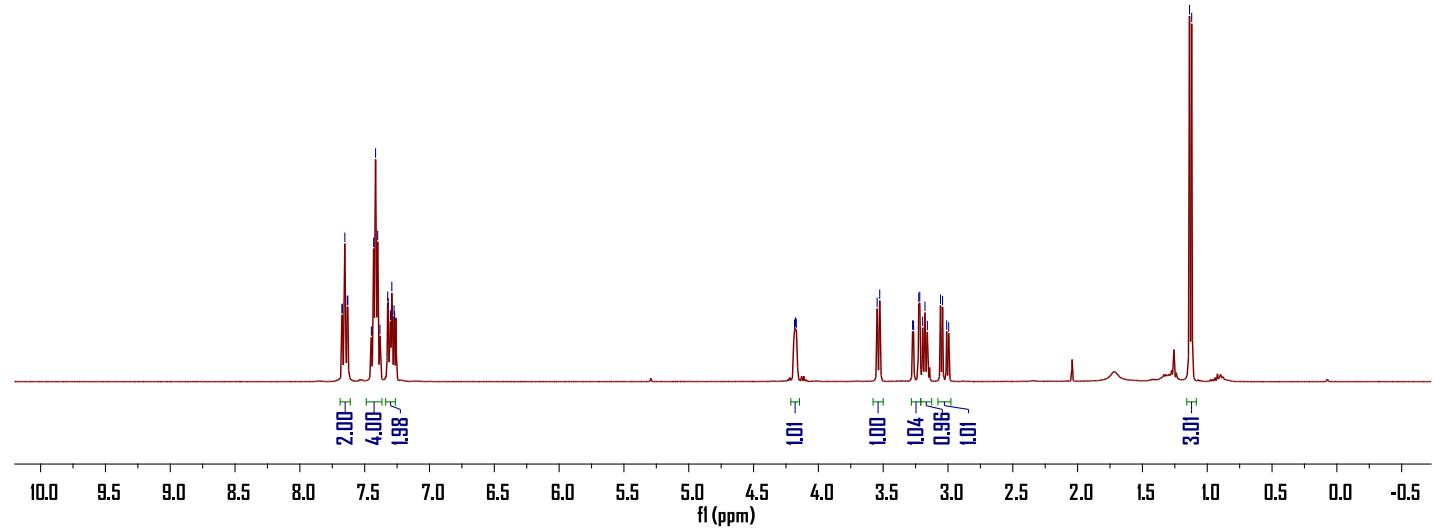

空嵒

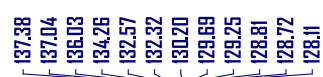

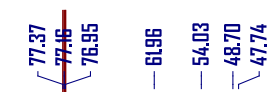

$\underset{\text { 品 }}{\text { | }}$

${ }^{13} \mathrm{C} \mathrm{NMR}\left(150 \mathrm{MHz}, \mathrm{CDCl}_{3}\right)$

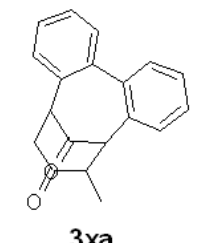

$3 \times a$

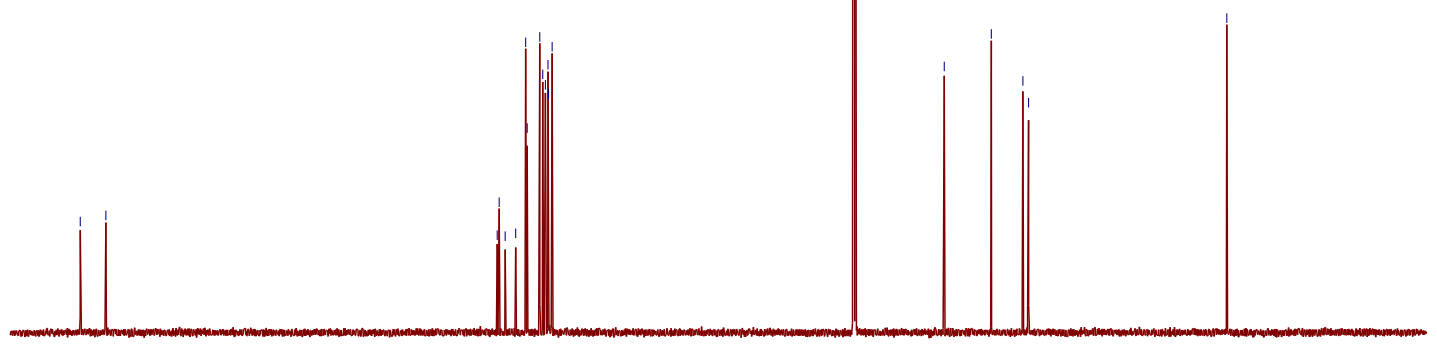

$\begin{array}{lllllllllllllllllllllll}210 & 200 & 190 & 180 & 170 & 160 & 150 & 140 & 130 & 120 & 110 & \underset{f l}{100}(\mathrm{ppm}) & 90 & 80 & 70 & 60 & 50 & 40 & 30 & 20 & 10 & 0 & -10\end{array}$ 


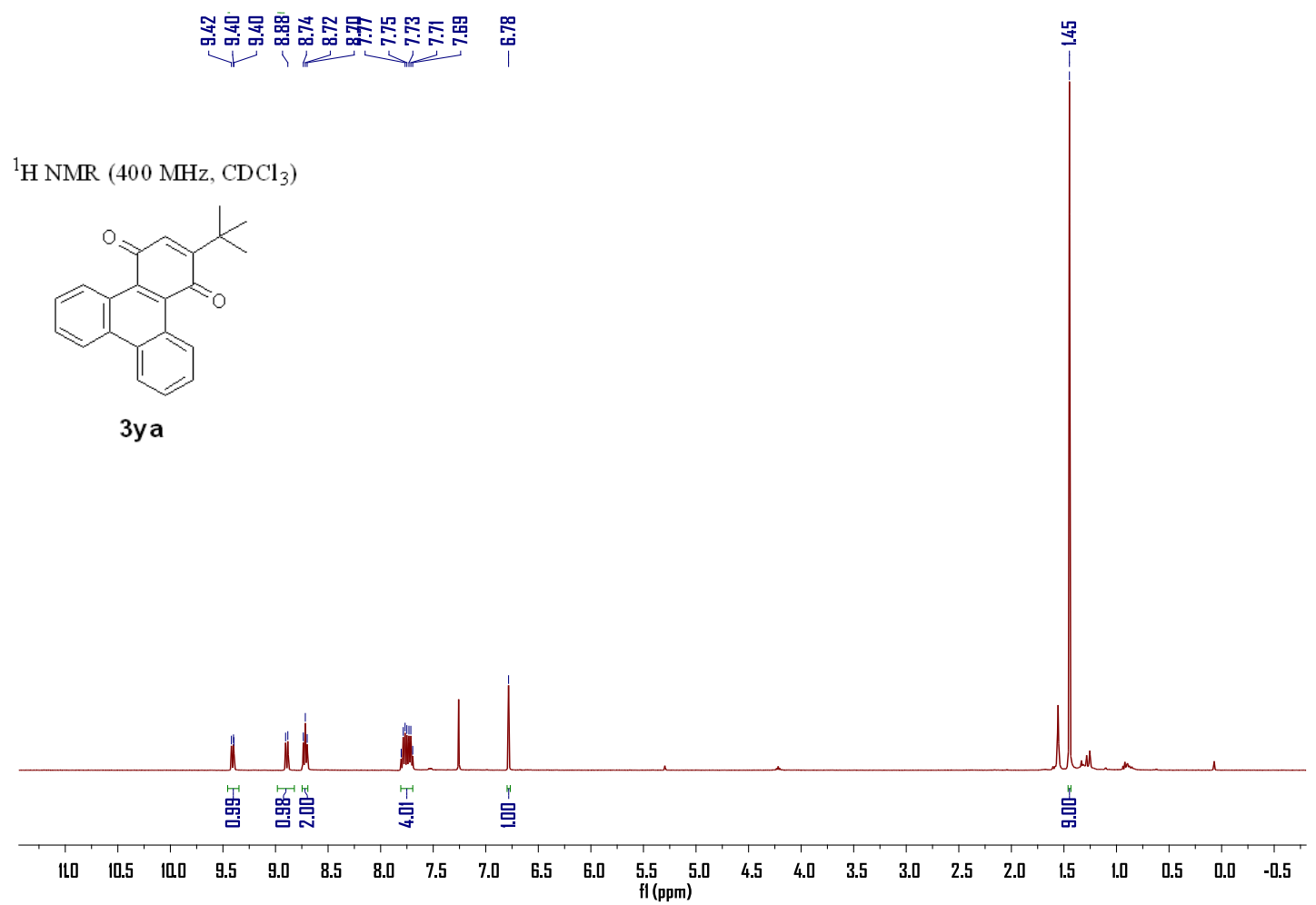

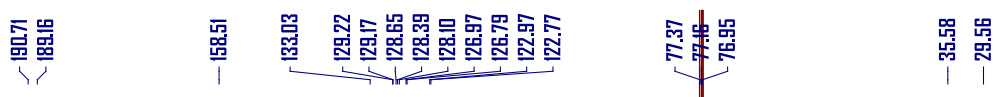

${ }^{13} \mathrm{CNMR}\left(150 \mathrm{MHz}, \mathrm{CDCl}_{3}\right)$

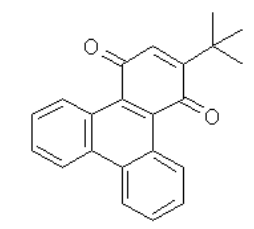

зуа

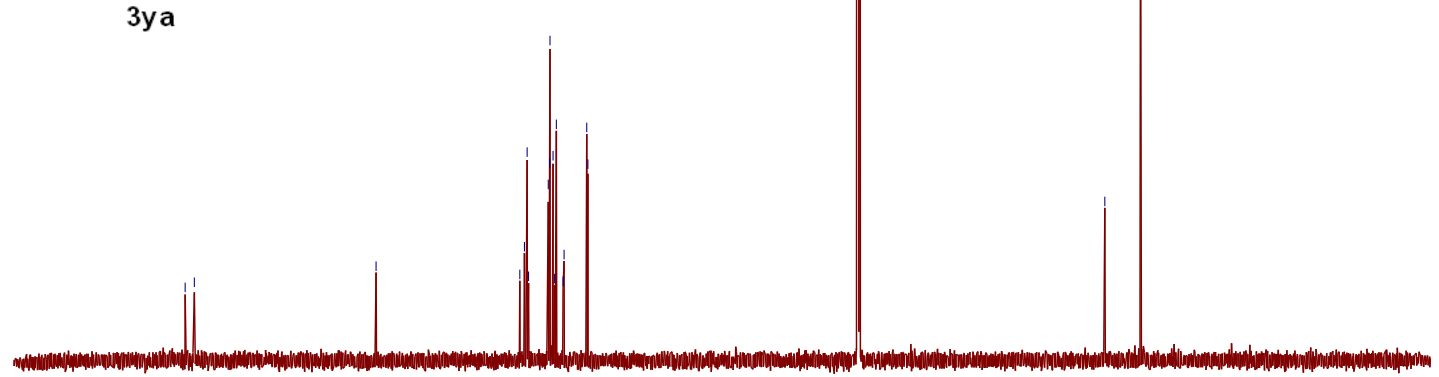

$\begin{array}{lllllllllllllllllllllll}210 & 200 & 190 & 180 & 170 & 160 & 150 & 140 & 130 & 120 & 110 & \begin{array}{c}100 \\ \mathrm{fl}(\mathrm{ppm})\end{array} & 90 & 80 & 70 & 60 & 50 & 40 & 30 & 20 & 10 & 0 & -10\end{array}$ 
${ }^{1} \mathrm{HNMR}\left(600 \mathrm{MHz}, \mathrm{CDCl}_{3}\right)$

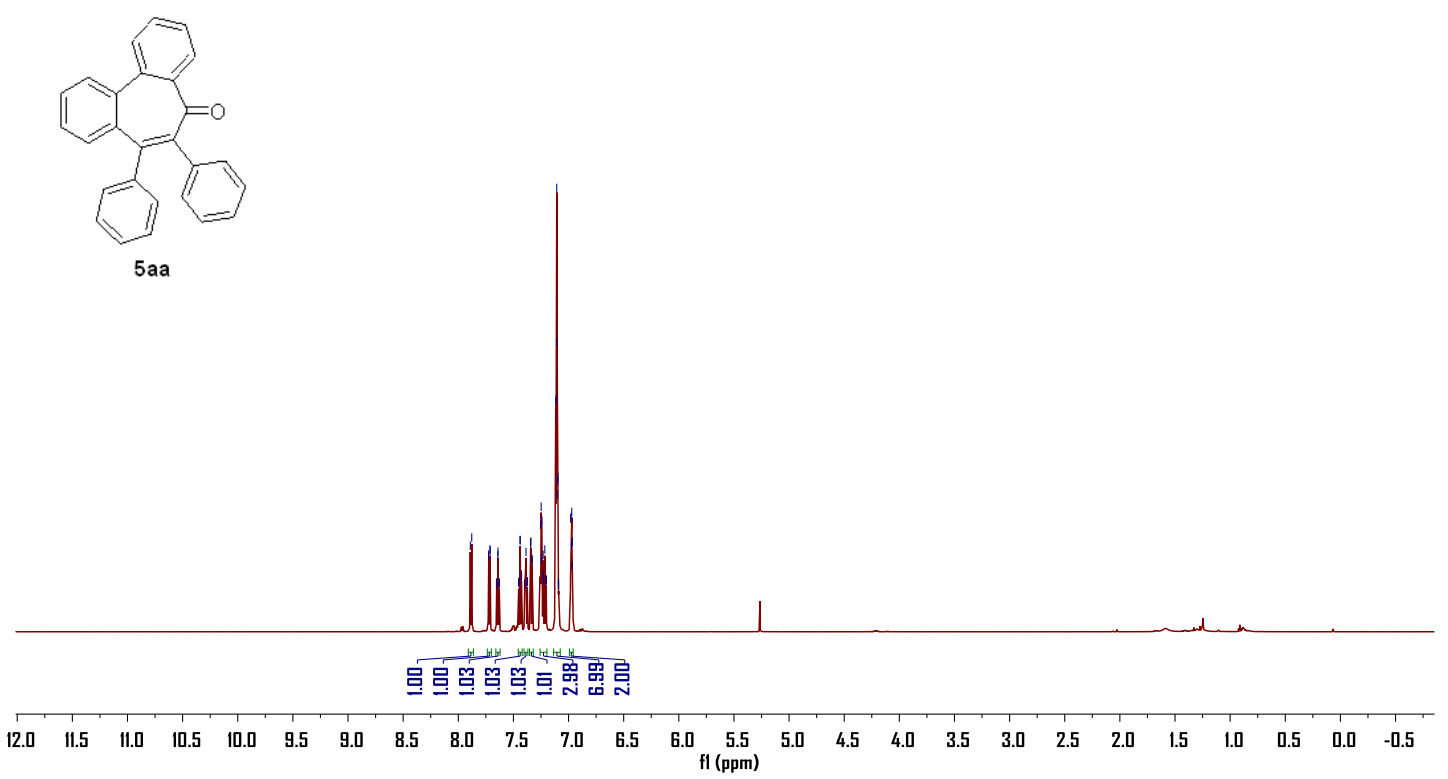

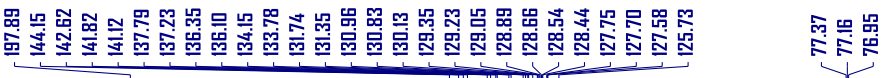

${ }^{13} \mathrm{C} \mathrm{NMR}\left(150 \mathrm{MHz}, \mathrm{CDCl}_{3}\right)$

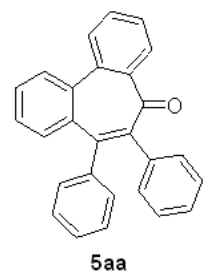

5 aa

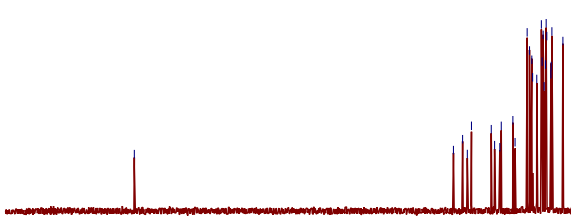

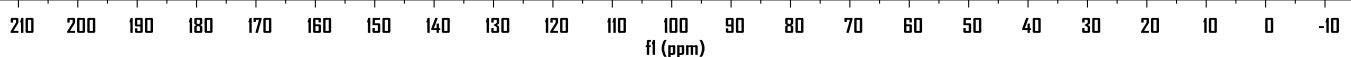


${ }^{1} \mathrm{H} \mathrm{NMR}\left(600 \mathrm{MHz}, \mathrm{CDCl}_{3}\right)$
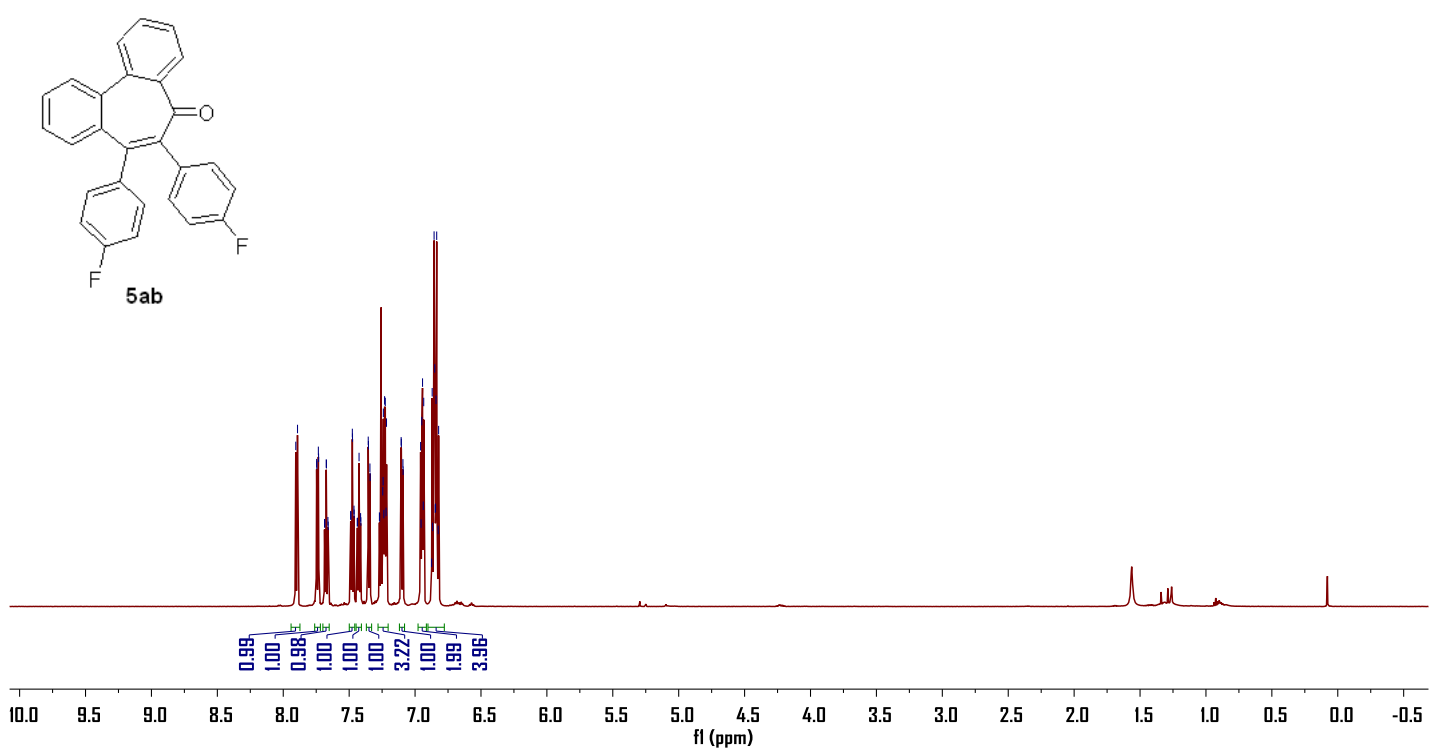

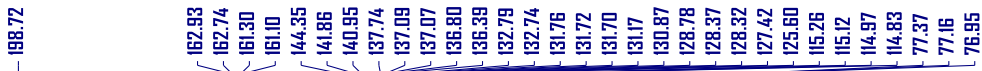

${ }^{13} \mathrm{C} \mathrm{NMR}\left(150 \mathrm{MHz}, \mathrm{CDCl}_{3}\right)$
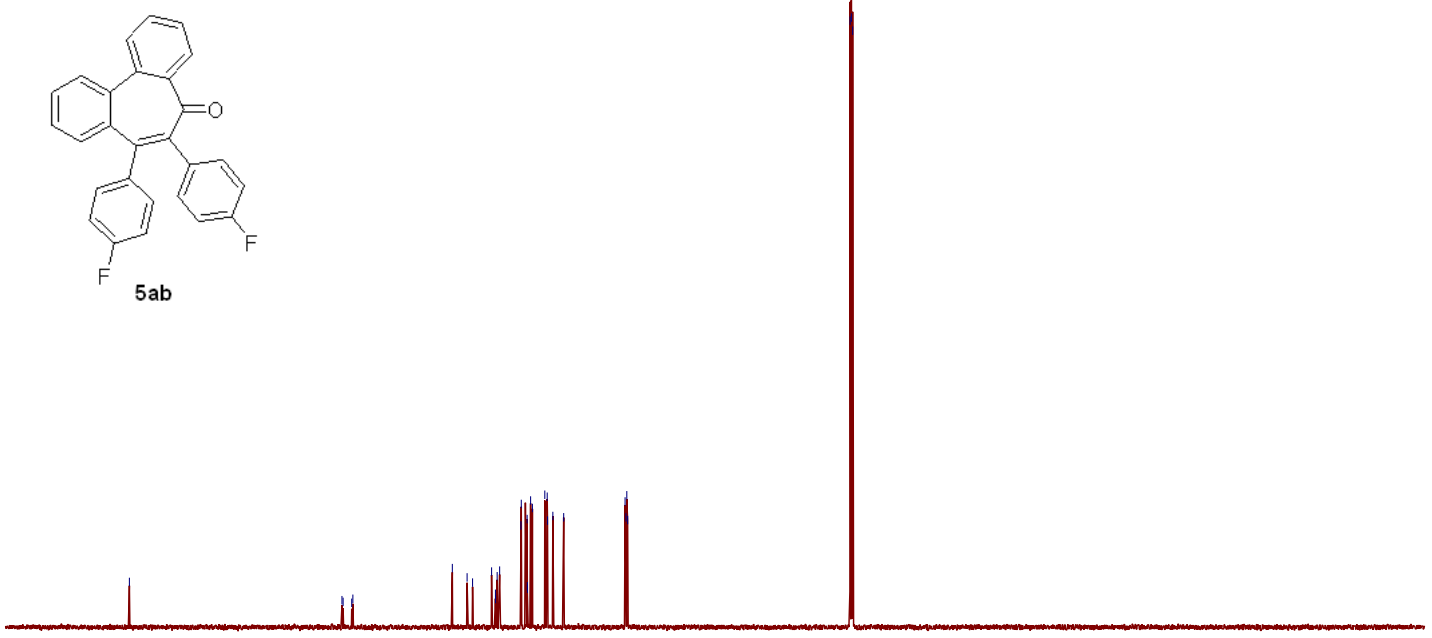

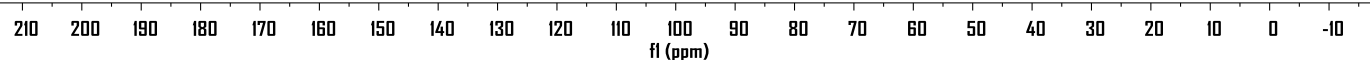


${ }^{1} \mathrm{HNMR}\left(600 \mathrm{MHz}, \mathrm{CDCl}_{3}\right)$
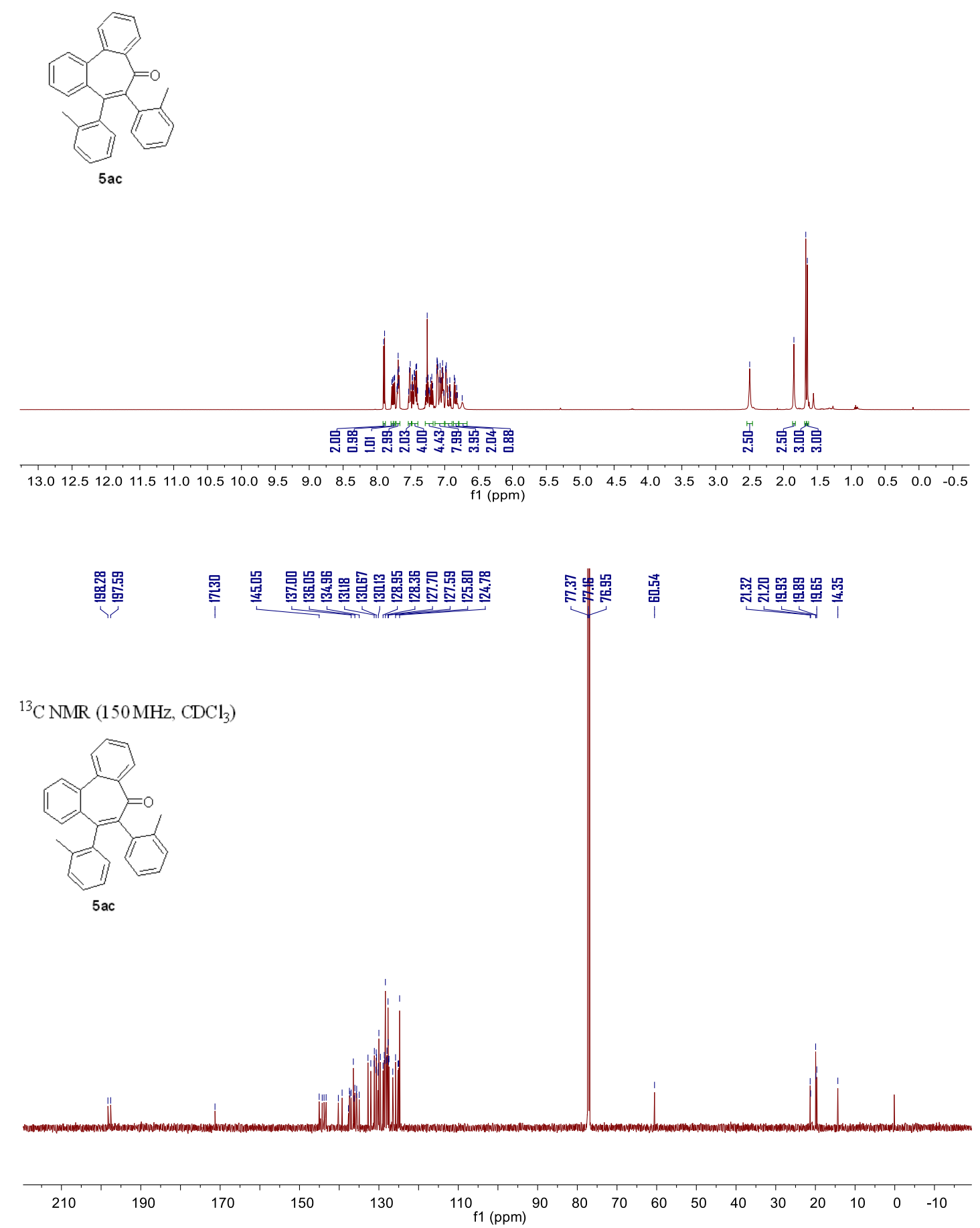


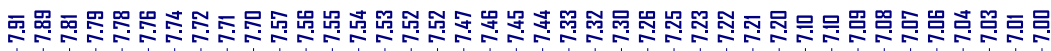

${ }^{1} \mathrm{HNMR}\left(600 \mathrm{MHz}, \mathrm{CDCl}_{3}\right)$

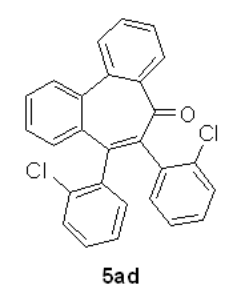

5 ad

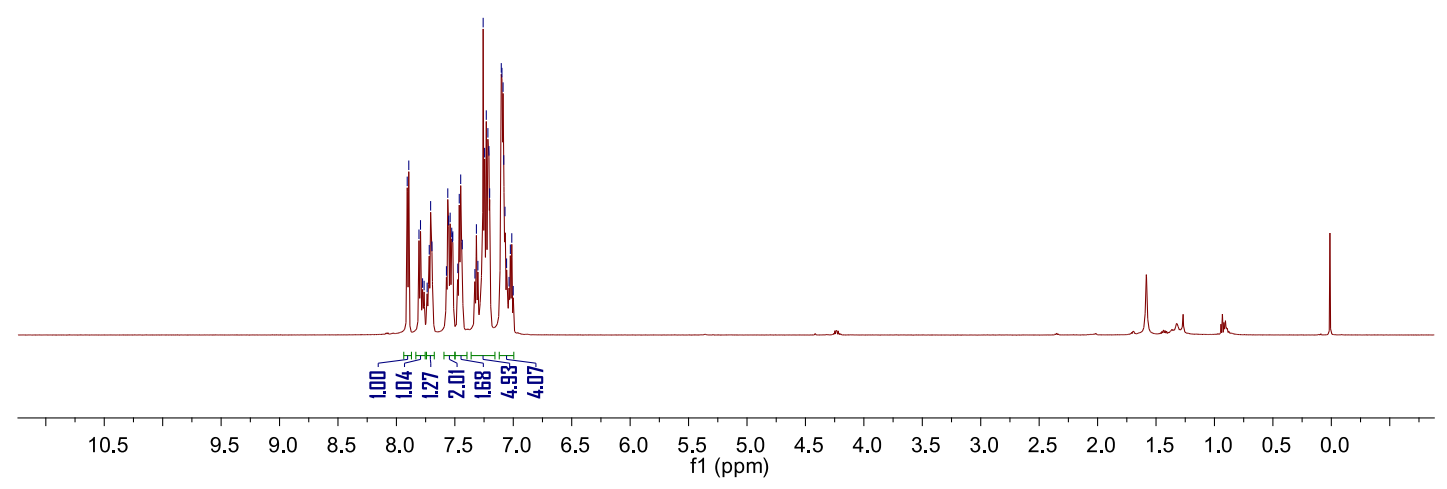

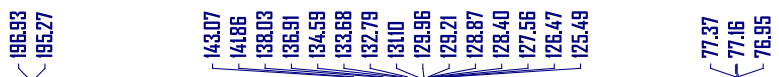

${ }^{13} \mathrm{C} \mathrm{NMR}\left(150 \mathrm{MHz}, \mathrm{CDCl}_{3}\right)$

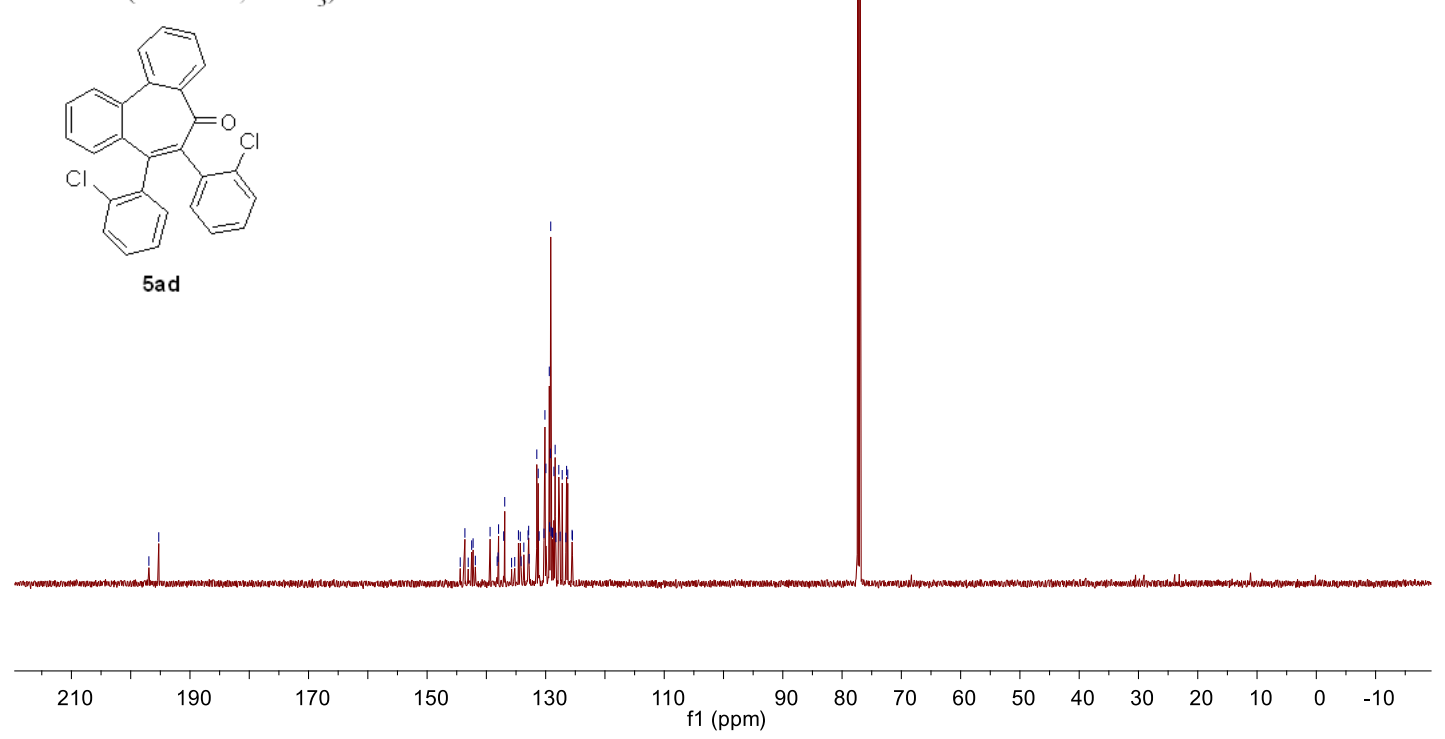


${ }^{1} \mathrm{HNMR}\left(400 \mathrm{MHz}, \mathrm{CDCl}_{3}\right)$
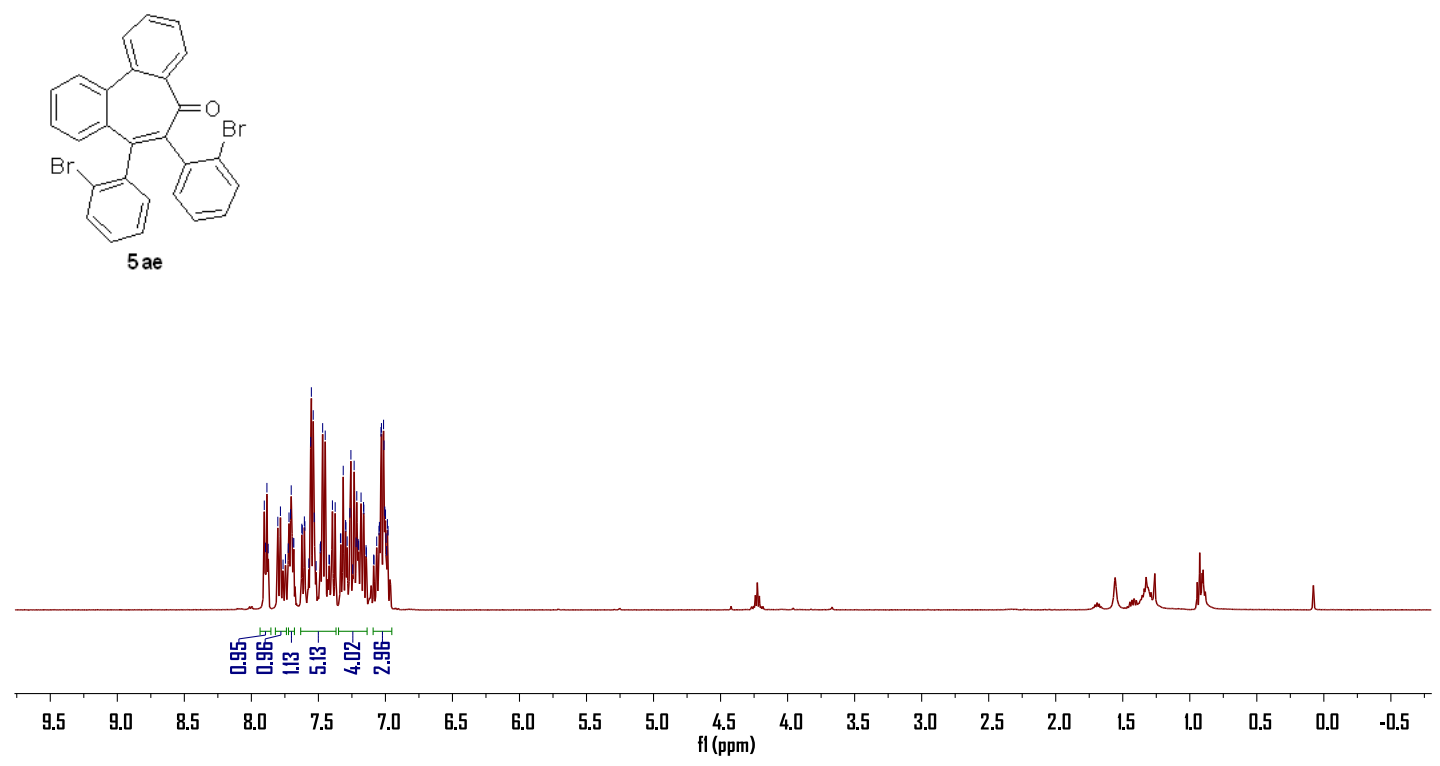

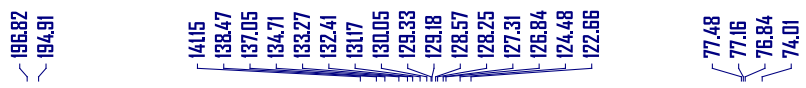
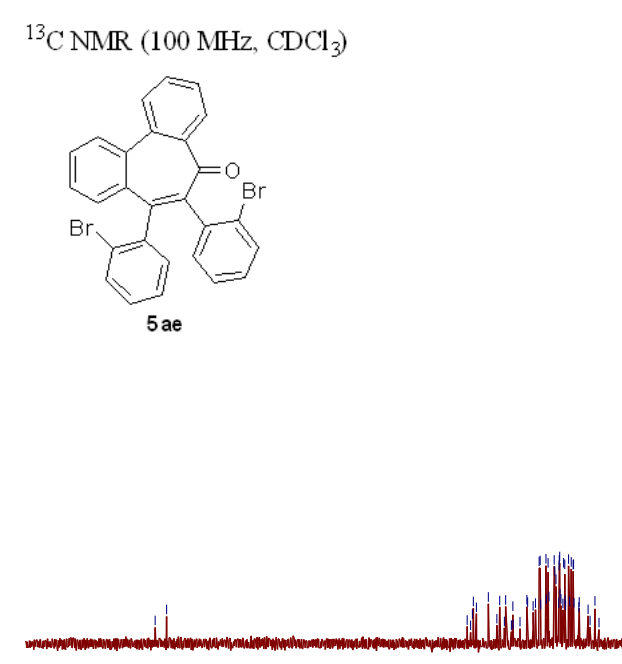

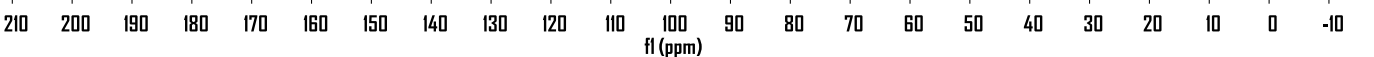


${ }^{1} \mathrm{HNMR}\left(400 \mathrm{MHz}, \mathrm{CDCl}_{3}\right)$

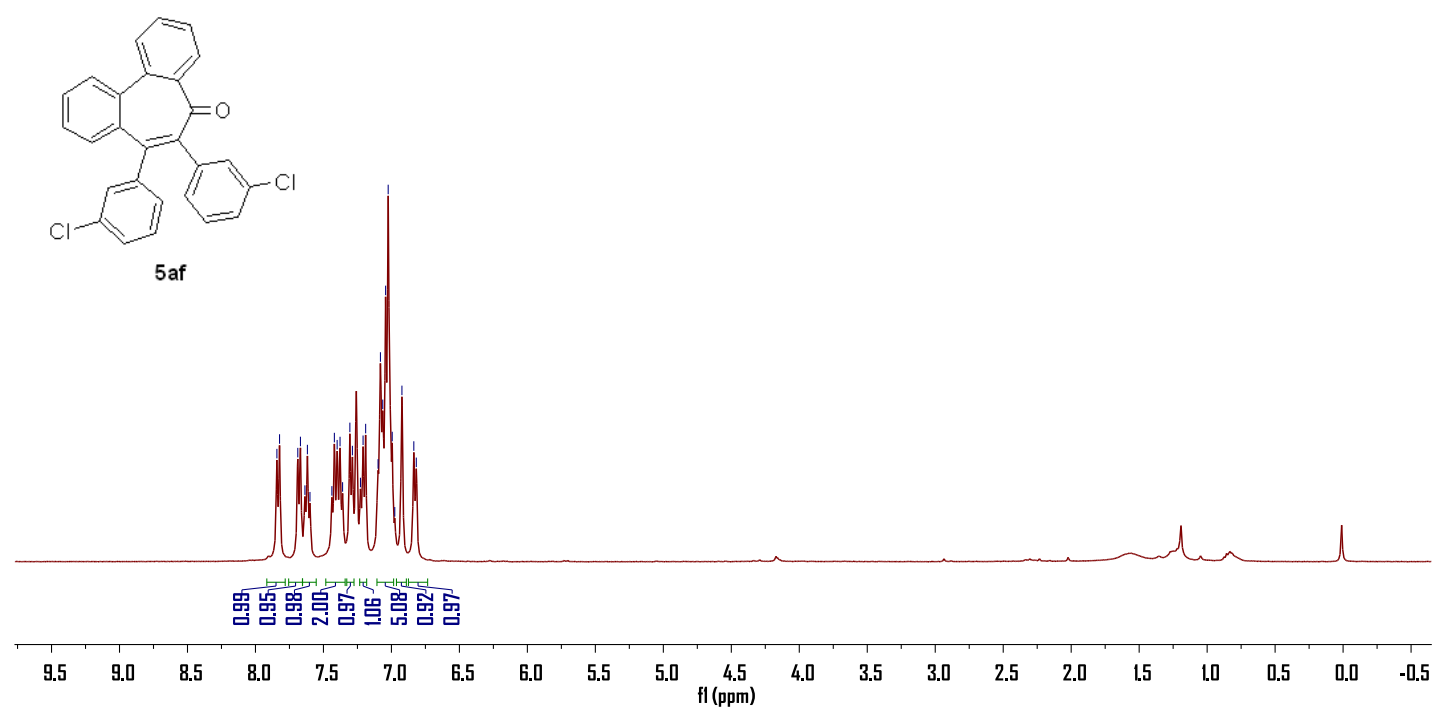

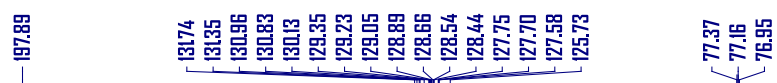

${ }^{13} \mathrm{CNMR}\left(150 \mathrm{MHz}, \mathrm{CDCl}_{3}\right)$
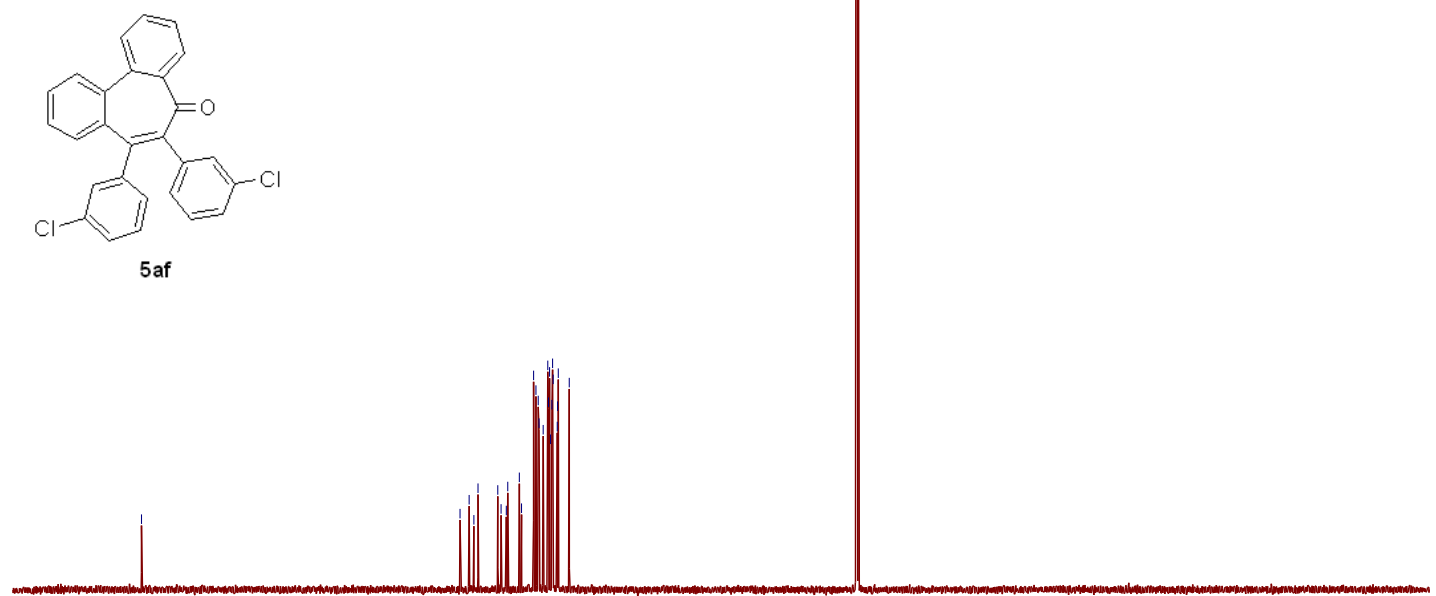

$\begin{array}{lllllllllllllllllllllll}210 & 200 & 190 & 180 & 170 & 160 & 150 & 140 & 130 & 120 & 110 & \begin{array}{c}100 \\ \mathrm{fl}(\mathrm{pm})\end{array} & 90 & 80 & 70 & 60 & 50 & 40 & 30 & 20 & 10 & 0 & -10\end{array}$ 
${ }^{1} \mathrm{HNMR}\left(400 \mathrm{MHz}, \mathrm{CDCl}_{3}\right)$

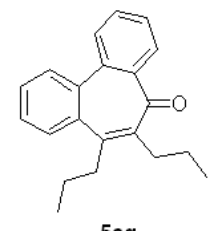

5 ag

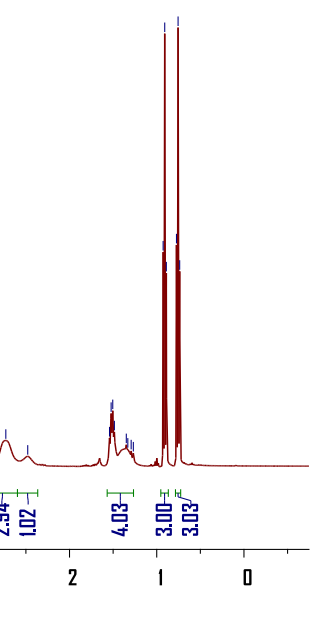

高

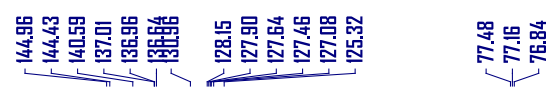

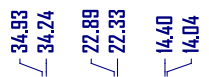

${ }^{13} \mathrm{C} \mathrm{NMR}\left(100 \mathrm{MHz}, \mathrm{CDCl}_{3}\right)$

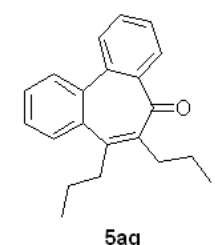

5 ag

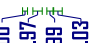

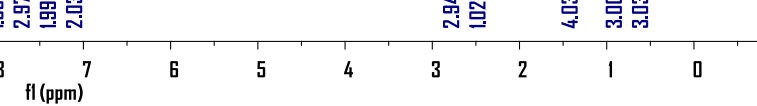

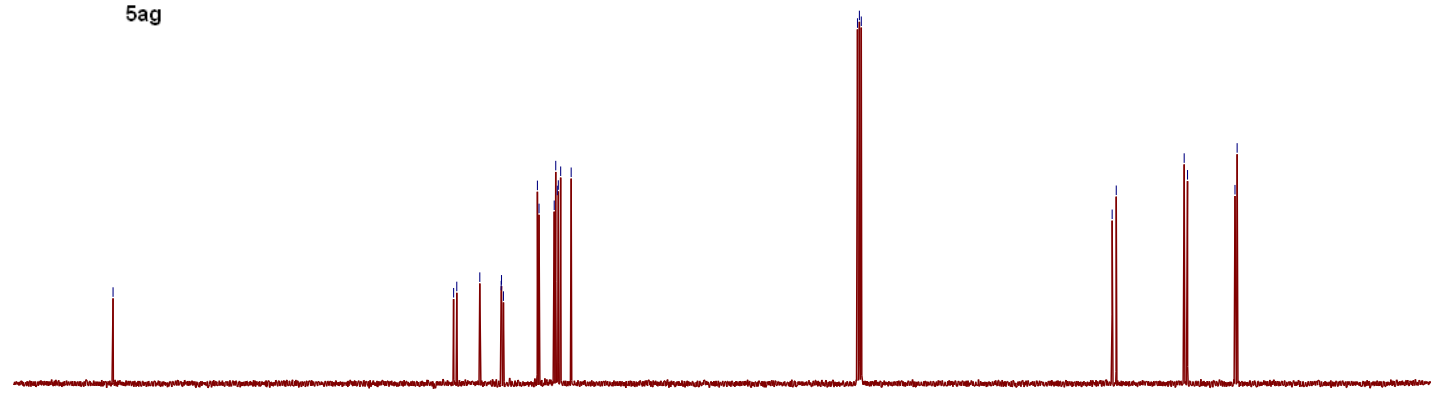

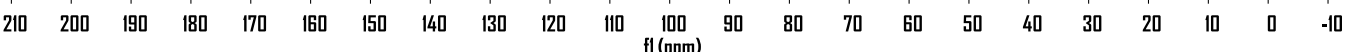




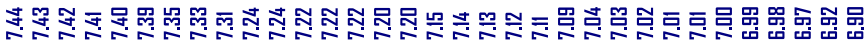

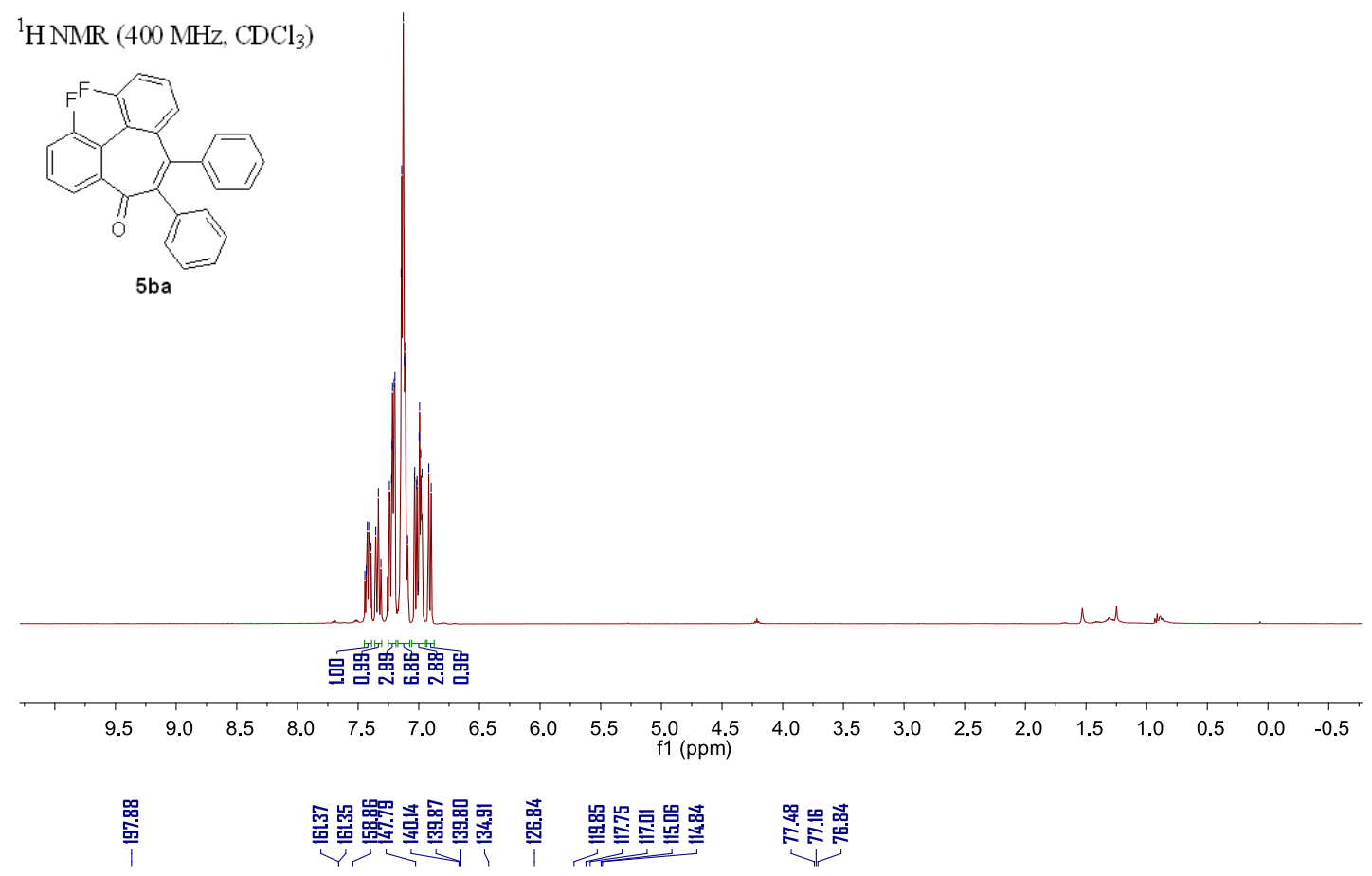

${ }^{13} \mathrm{CNMR}\left(100 \mathrm{MHz}, \mathrm{CDCl}_{3}\right)$

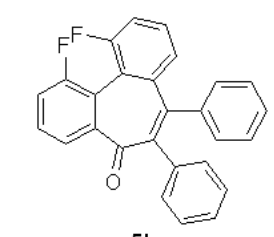

$5 \mathrm{ba}$

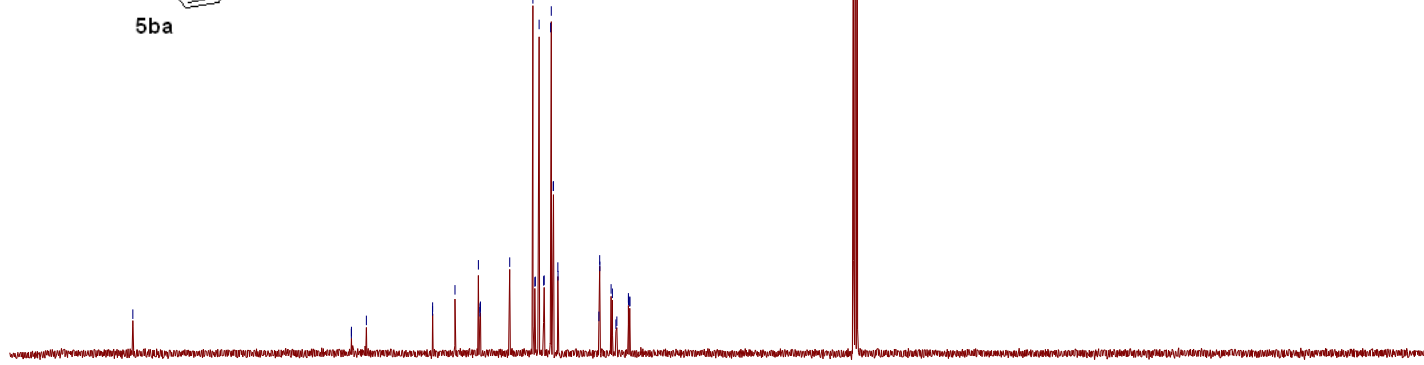

$\begin{array}{lllllllllllllllllllllll}210 & 200 & 190 & 180 & 170 & 160 & 150 & 140 & 130 & 120 & 110 \underset{f 1}{100}(\mathrm{ppm}) & 90 & 80 & 70 & 60 & 50 & 40 & 30 & 20 & 10 & 0 & -10\end{array}$ 


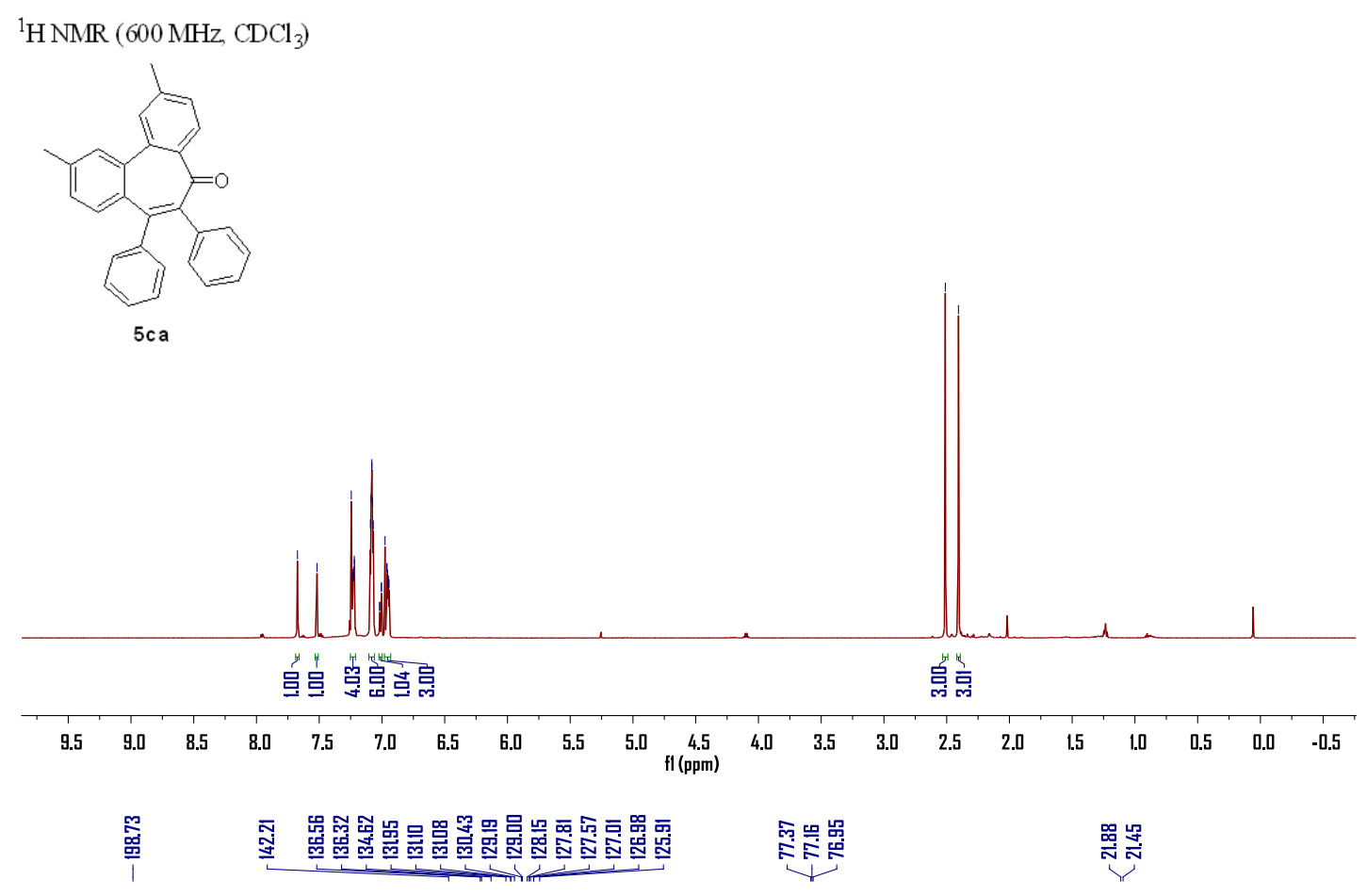

${ }^{13} \mathrm{CNMR}\left(150 \mathrm{MHz}, \mathrm{CDCl}_{3}\right)$

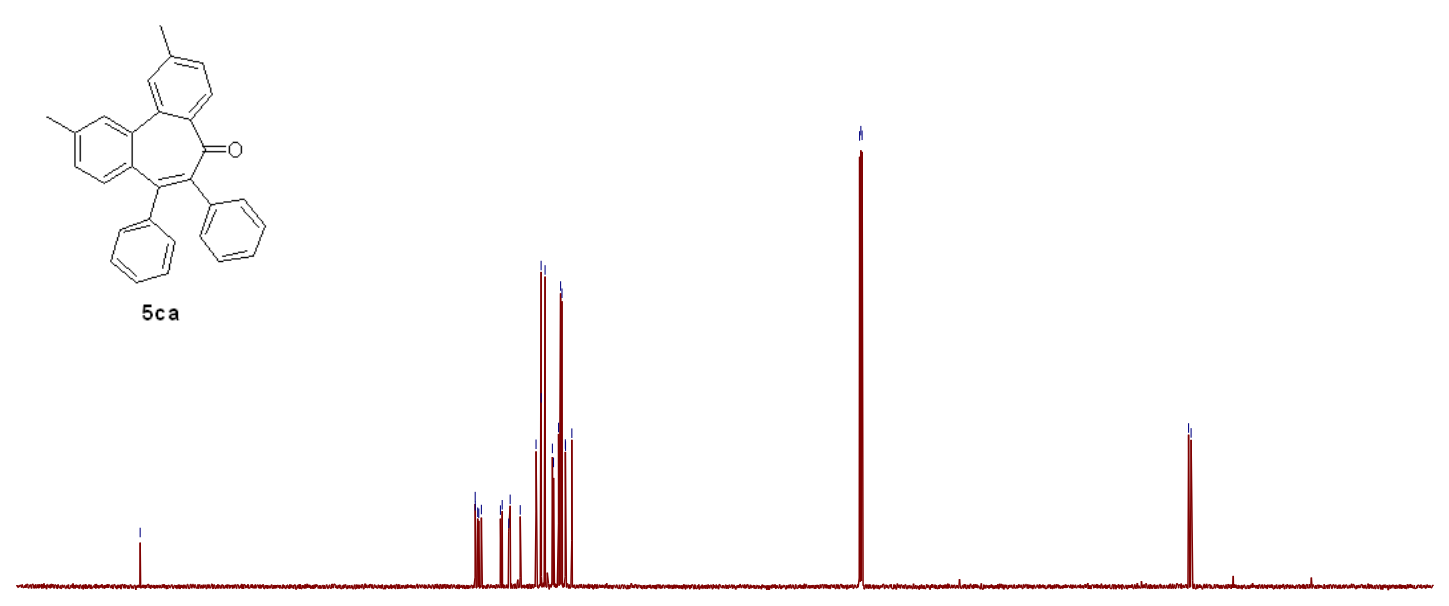

$\begin{array}{lllllllllllllllllllllll}210 & 200 & 190 & 180 & 170 & 160 & 150 & 140 & 130 & 120 & 110 & \begin{array}{c}100 \\ \mathrm{fl}(\mathrm{ppm})\end{array} & 90 & 80 & 70 & 60 & 50 & 40 & 30 & 20 & 10 & 0 & -10\end{array}$ 


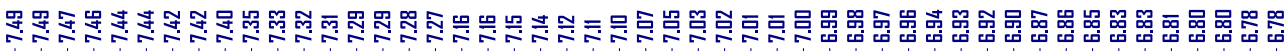

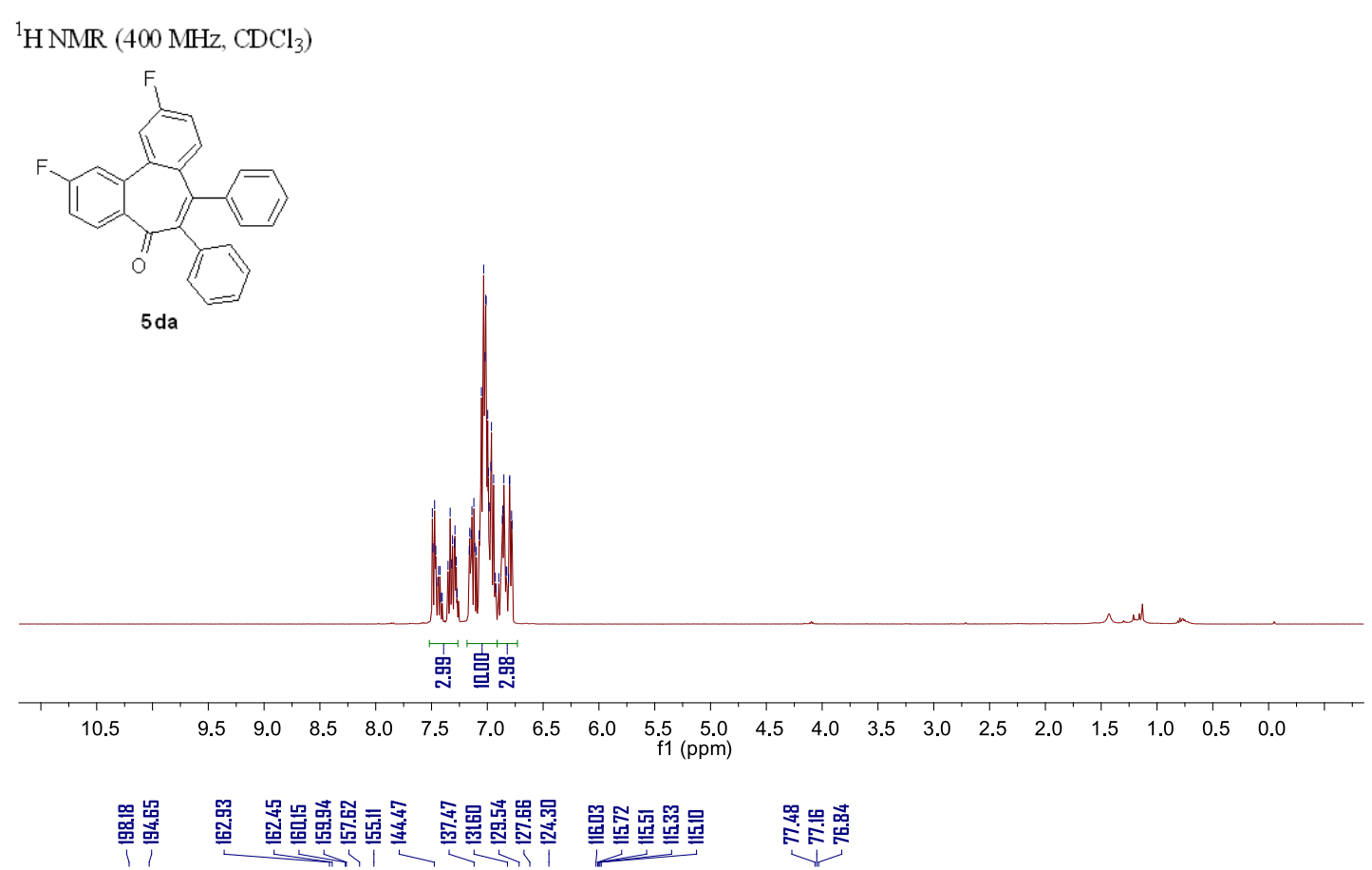

${ }^{13} \mathrm{C} \mathrm{NMR}\left(100 \mathrm{MHz}, \mathrm{CDCl}_{3}\right)$

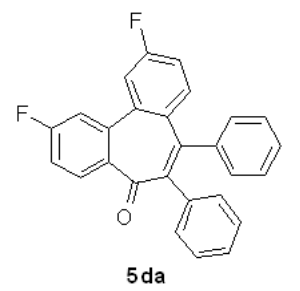

5 da

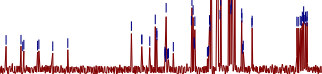

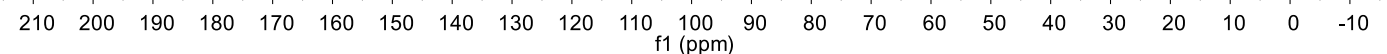




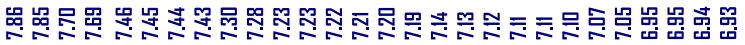

${ }^{1} \mathrm{HNMR}\left(400 \mathrm{MHz}, \mathrm{CDCl}_{3}\right)$

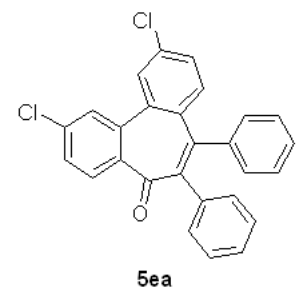

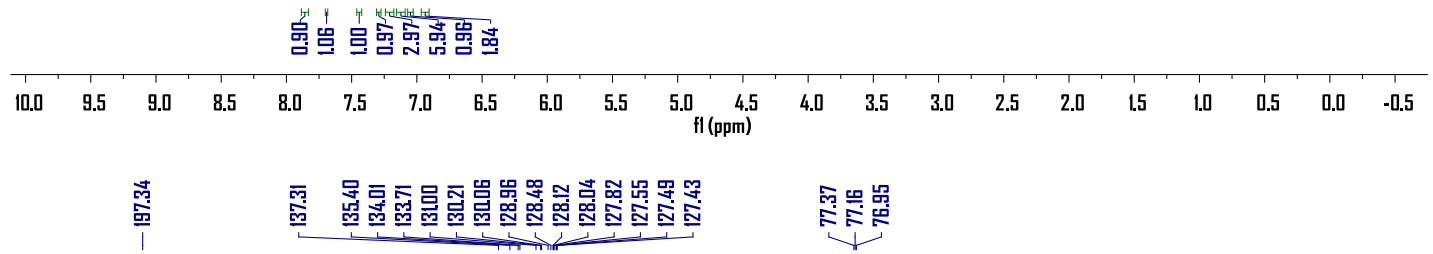

${ }^{13} \mathrm{C} \mathrm{NMR}\left(150 \mathrm{MHz}, \mathrm{CDCl}_{3}\right)$

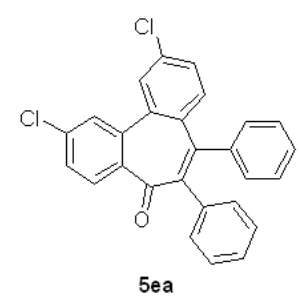

5ea

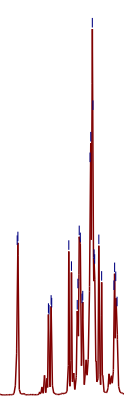




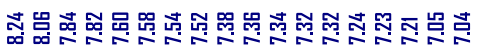

${ }^{1} \mathrm{HNMR}\left(400 \mathrm{MHz}, \mathrm{CDCl}_{3}\right)$

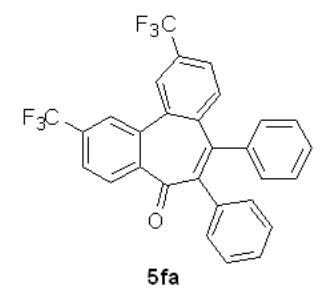

$5 \mathrm{fa}$

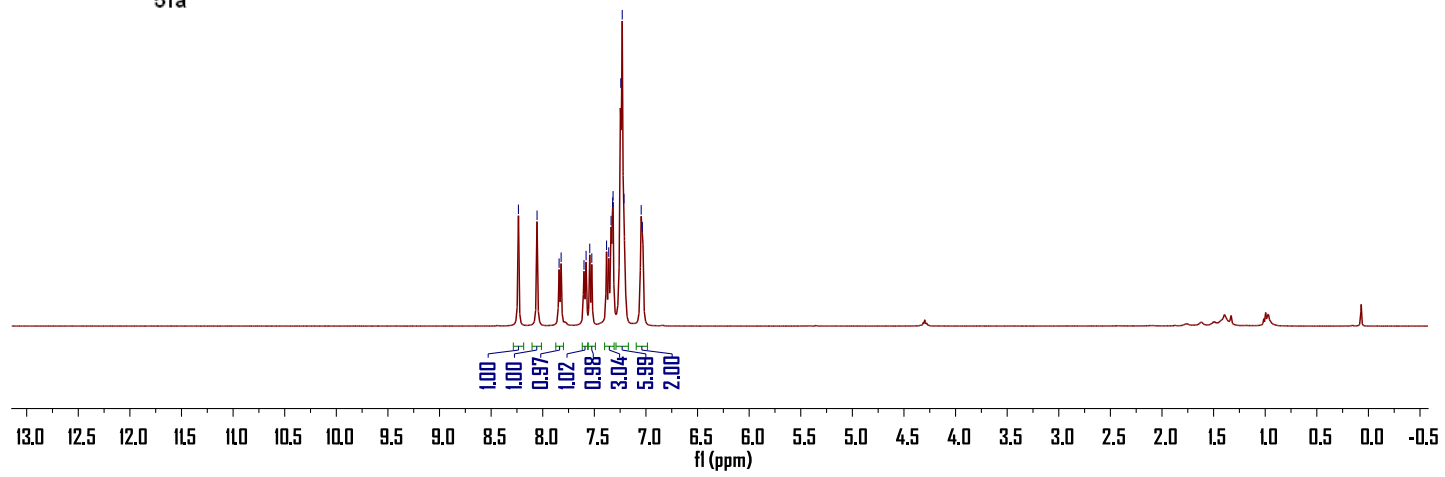

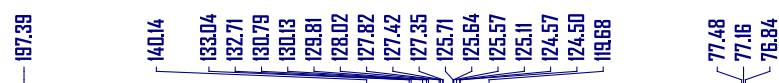

${ }^{13} \mathrm{CNMR}\left(100 \mathrm{MHz}, \mathrm{CDCl}_{3}\right)$

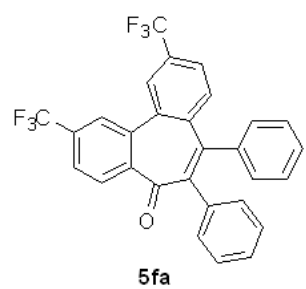

$5 \mathrm{fa}$

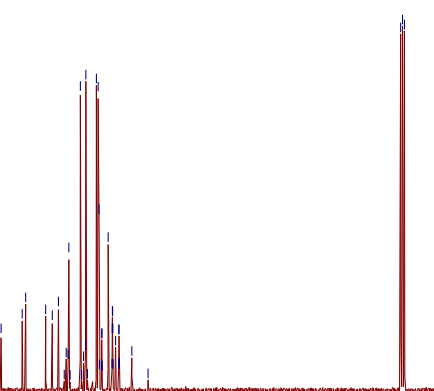

$\begin{array}{lllllllllllllllllllllll}210 & 200 & 190 & 180 & 170 & 160 & 150 & 140 & 130 & 120 & 110 & \underset{f 1}{(\mathrm{ppm})} & 90 & 80 & 70 & 60 & 50 & 40 & 30 & 20 & 10 & 0 & -10\end{array}$ 
${ }^{1} \mathrm{HNMR}\left(400 \mathrm{MHz}, \mathrm{CDCl}_{3}\right)$
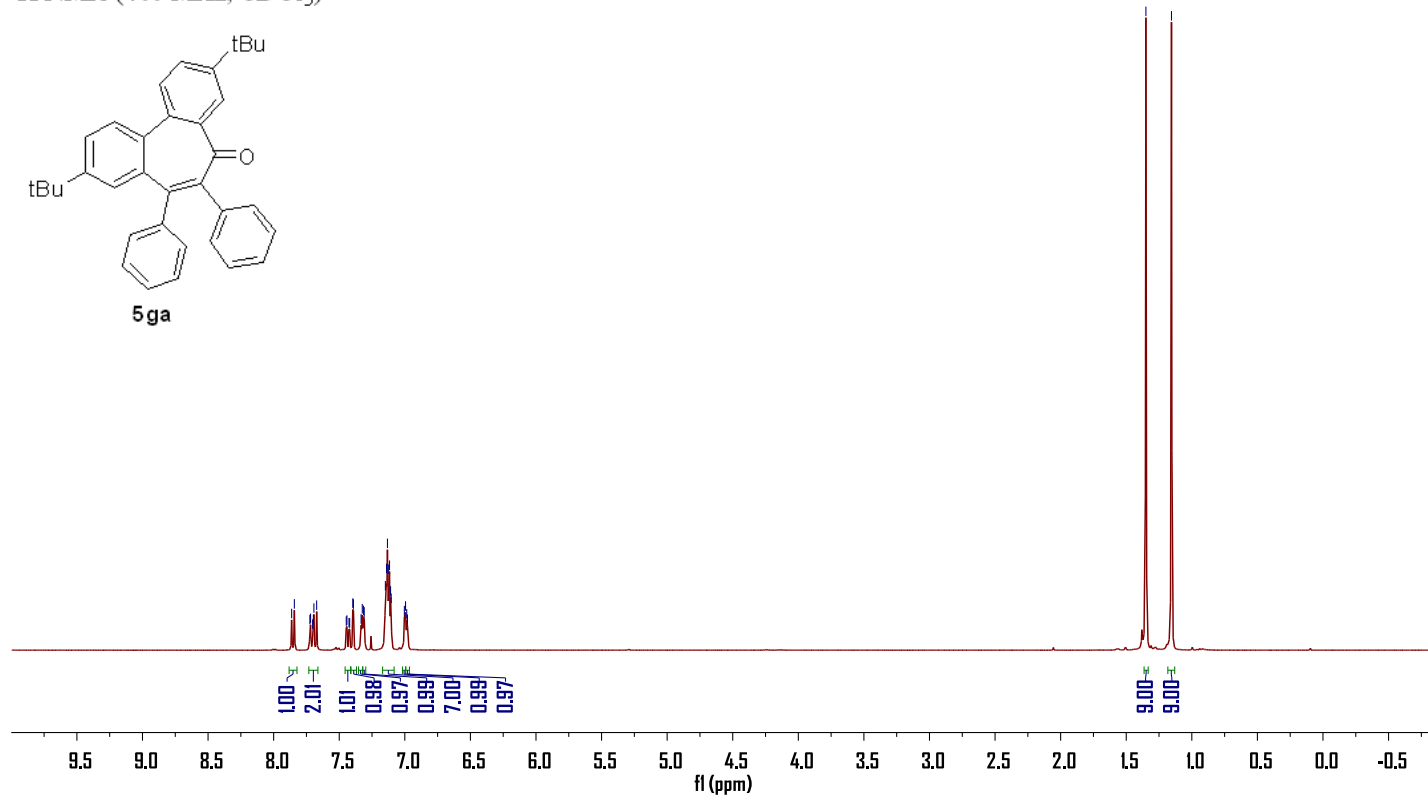

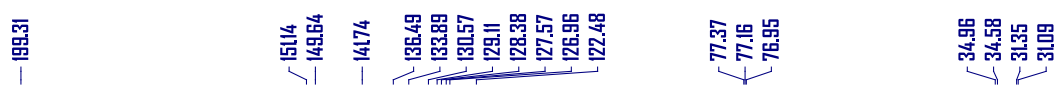

${ }^{13} \mathrm{CNMR}\left(150 \mathrm{MHz}, \mathrm{CDCl}_{3}\right)$
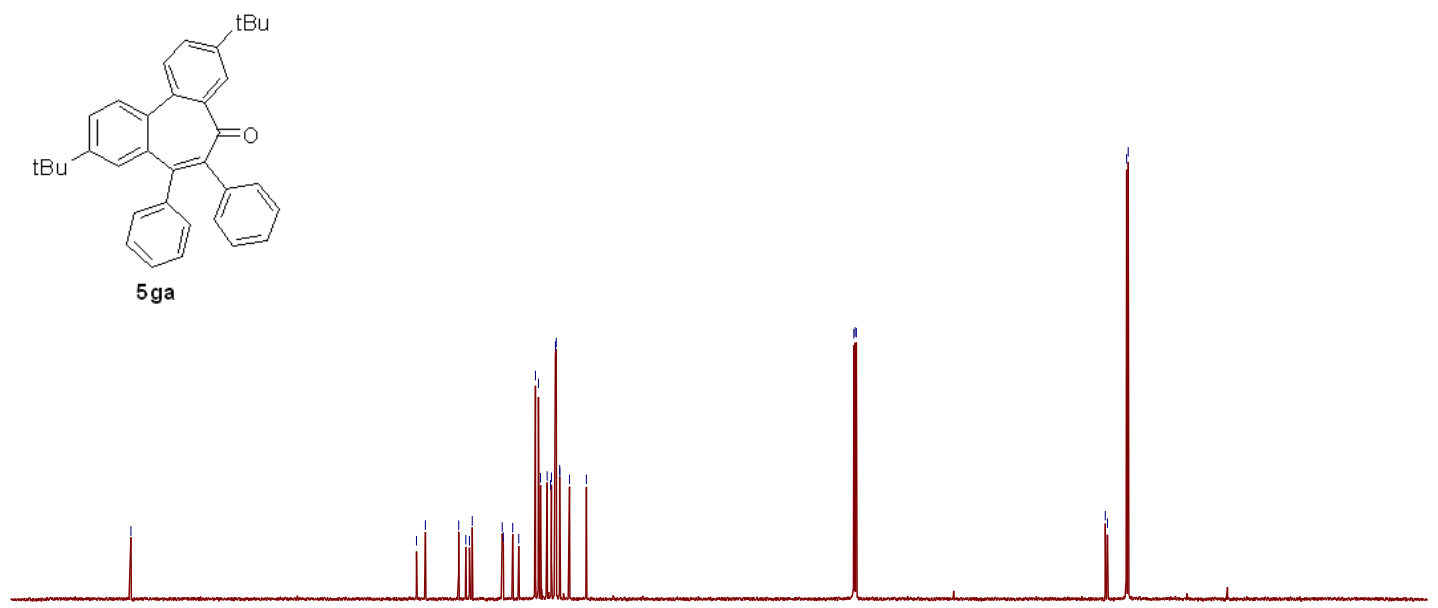

$\begin{array}{lllllllllllllllllllllll}210 & 200 & 190 & 180 & 170 & 160 & 150 & 140 & 130 & 120 & 110 & \begin{array}{c}100 \\ \mathrm{fl}(\mathrm{ppm})\end{array} & 90 & 80 & 70 & 60 & 50 & 40 & 30 & 20 & 10 & 0 & -10\end{array}$ 


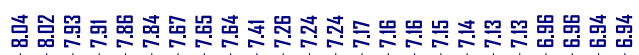

${ }^{1} \mathrm{HNMR}\left(400 \mathrm{MHz}, \mathrm{CDCl}_{3}\right)$

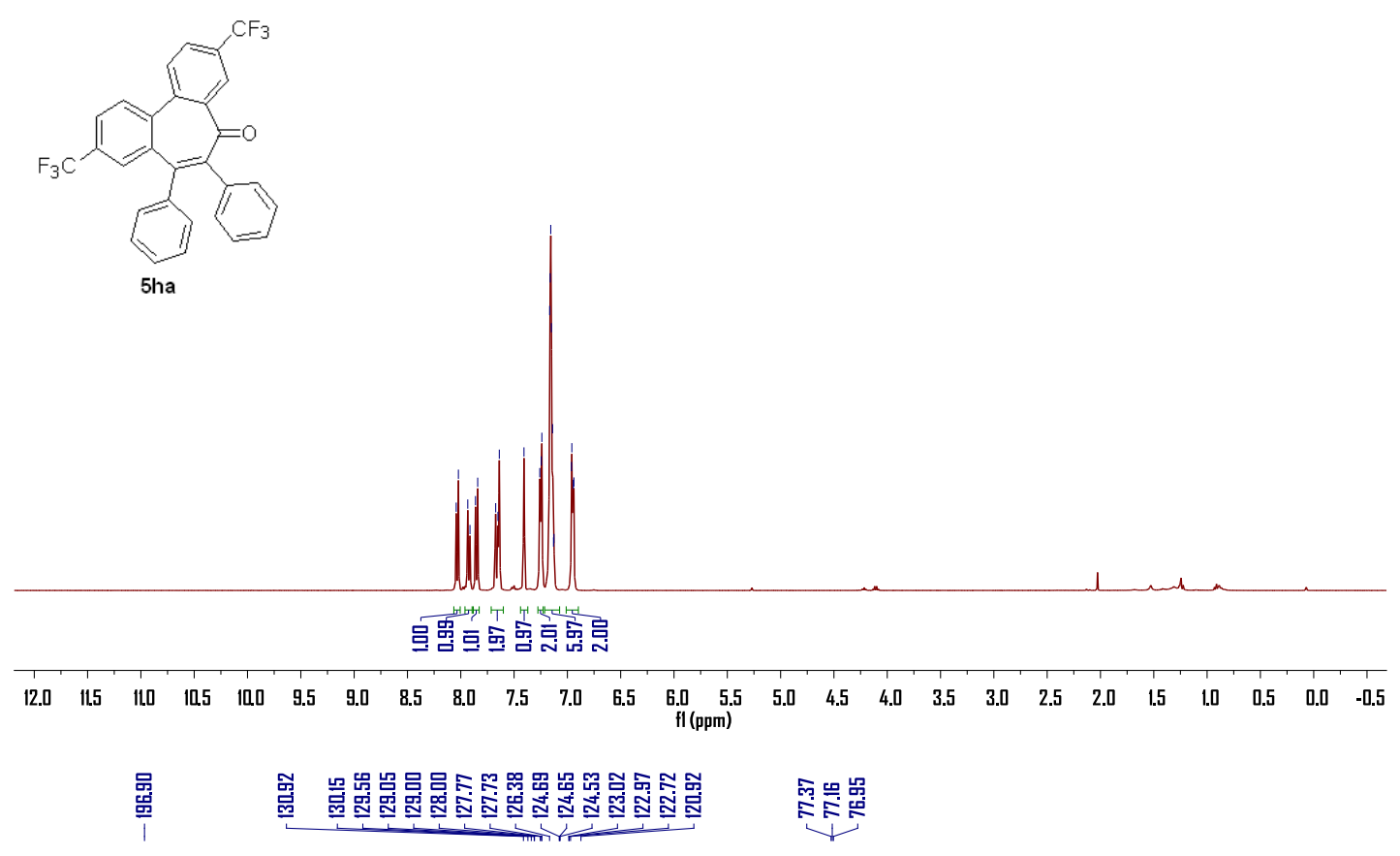

${ }^{13} \mathrm{C} \mathrm{NMR}\left(150 \mathrm{MHz}, \mathrm{CDCl}_{3}\right)$
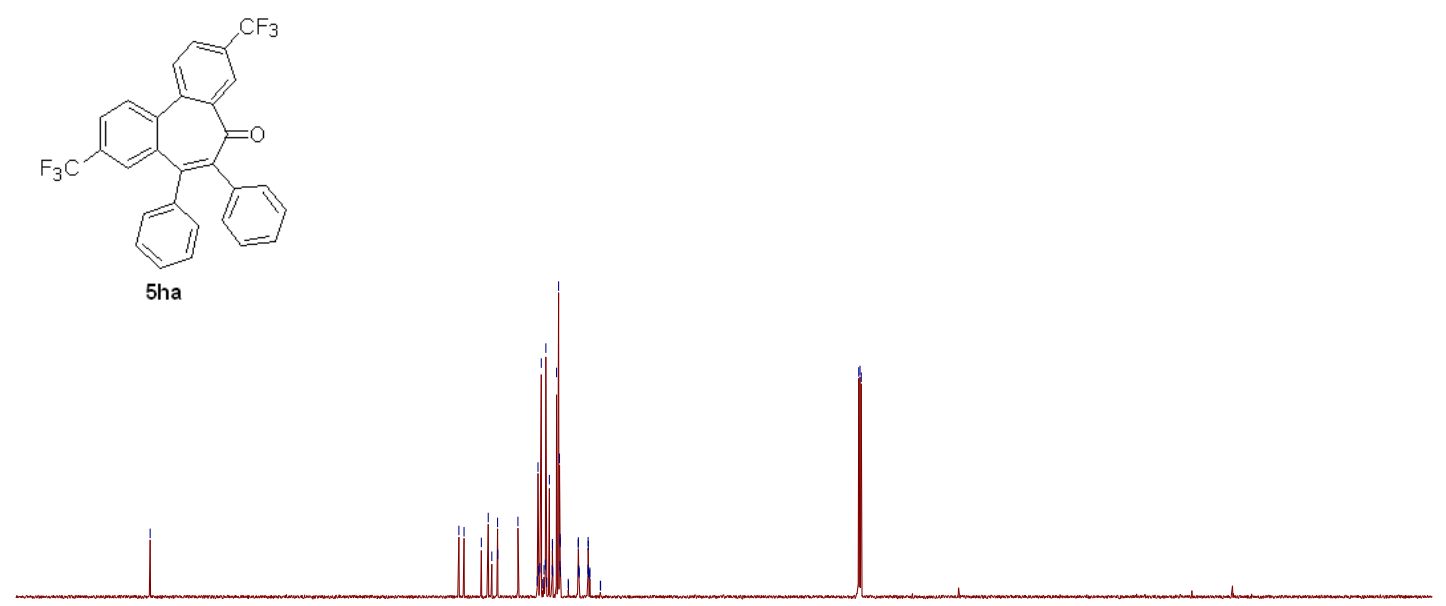

$\begin{array}{llllllllllllllllllll}210 & 190 & 170 & 150 & 130 & 110 & 90 & 80 & 70 & 60 & 50 & 40 & 30 & 20 & 10 & 0 & -10\end{array}$ 
${ }^{1} \mathrm{HNMR}\left(600 \mathrm{MHz}, \mathrm{CDCl}_{3}\right)$

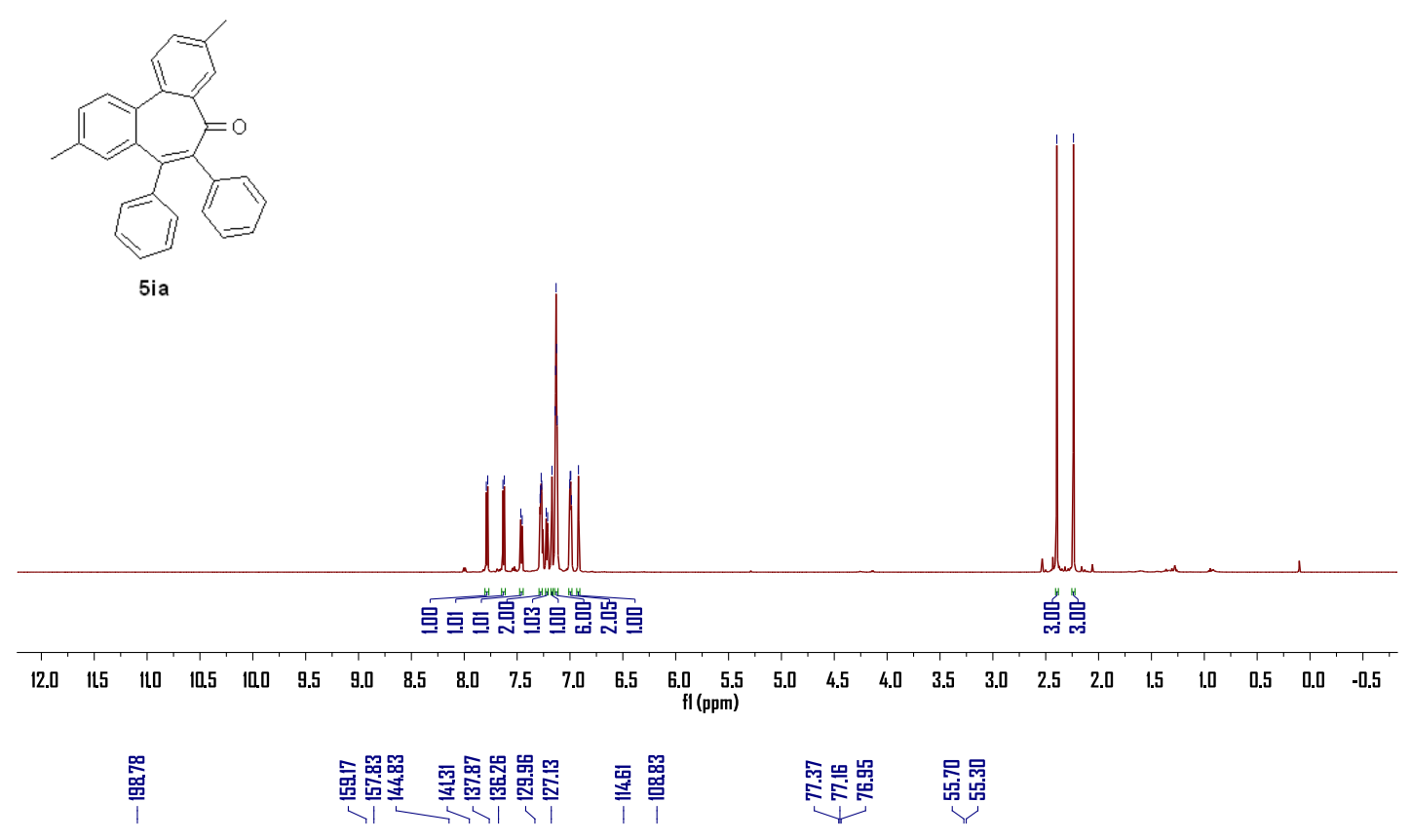

${ }^{13} \mathrm{C} \mathrm{NMR}\left(150 \mathrm{MHz}, \mathrm{CDCl}_{3}\right)$

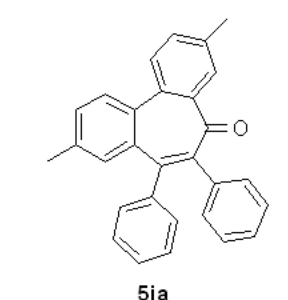

5 ia

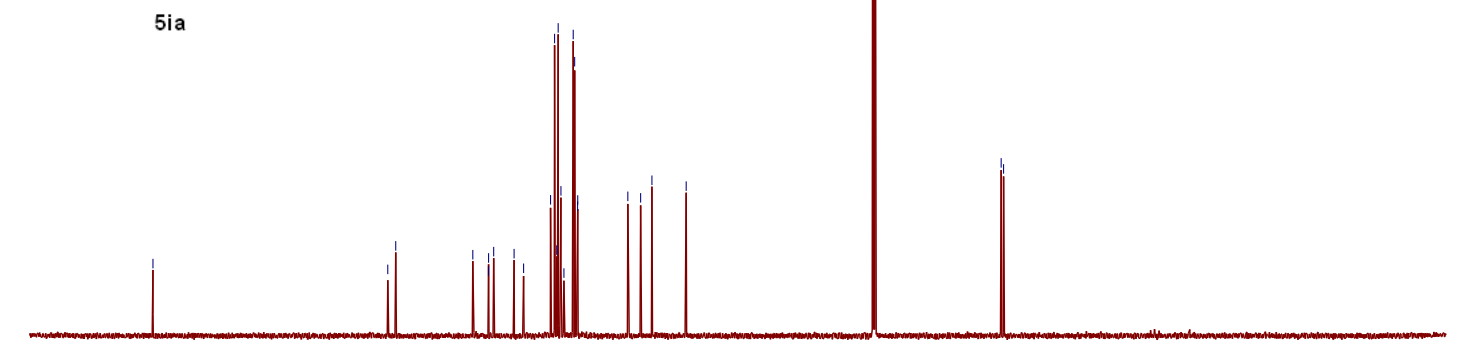

$\begin{array}{lllllllllllllllllllllll}210 & 200 & 190 & 180 & 170 & 160 & 150 & 140 & 130 & 120 & 110 & \begin{array}{c}100 \\ \mathrm{fl}(\mathrm{ppm})\end{array} & 90 & 80 & 70 & 60 & 50 & 40 & 30 & 20 & 10 & 0 & -10\end{array}$ 

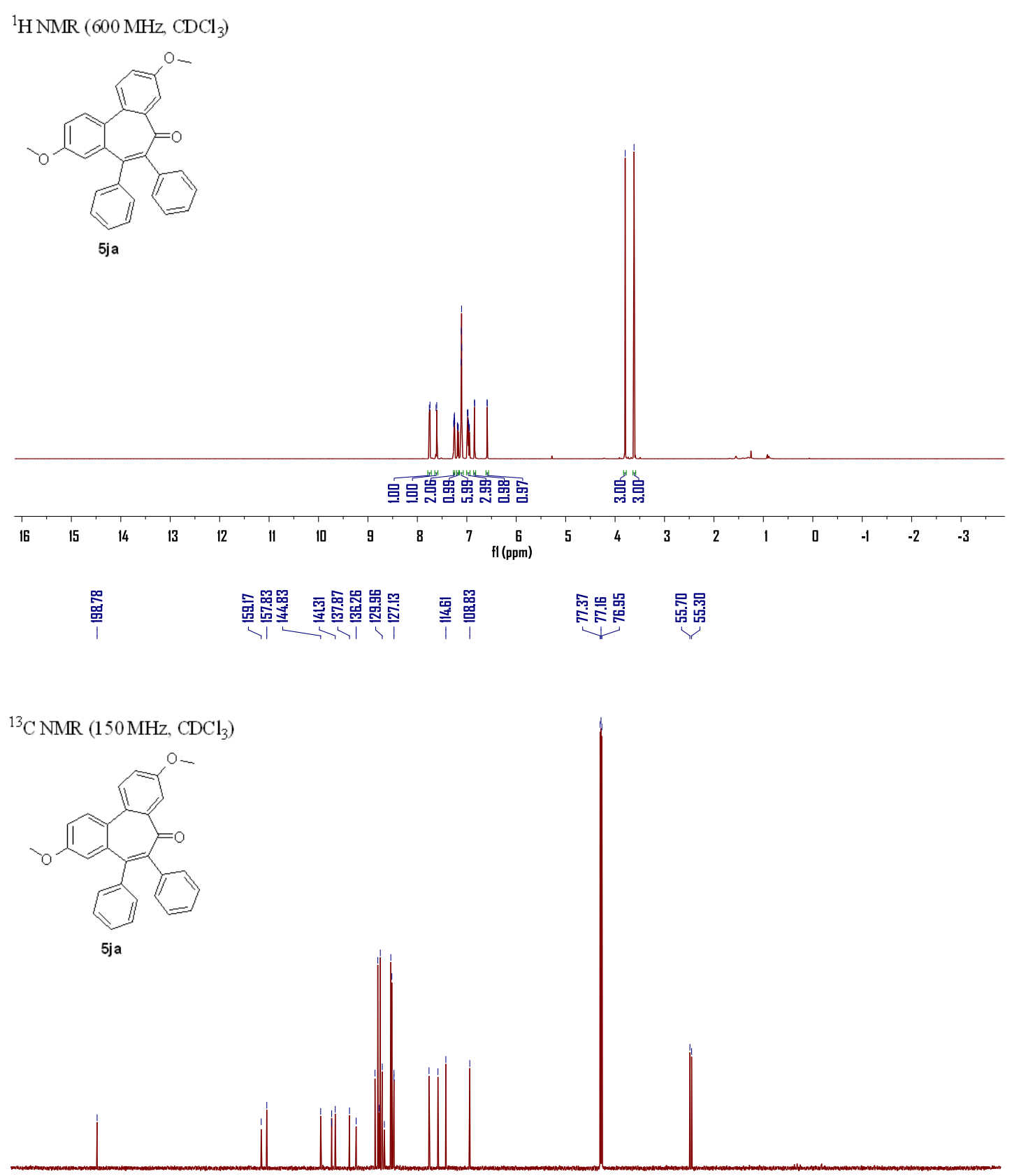

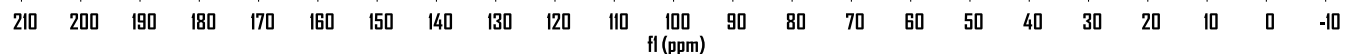




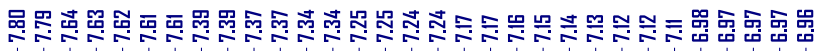

${ }^{1} \mathrm{HNMR}\left(600 \mathrm{MHz}, \mathrm{CDCl}_{3}\right)$

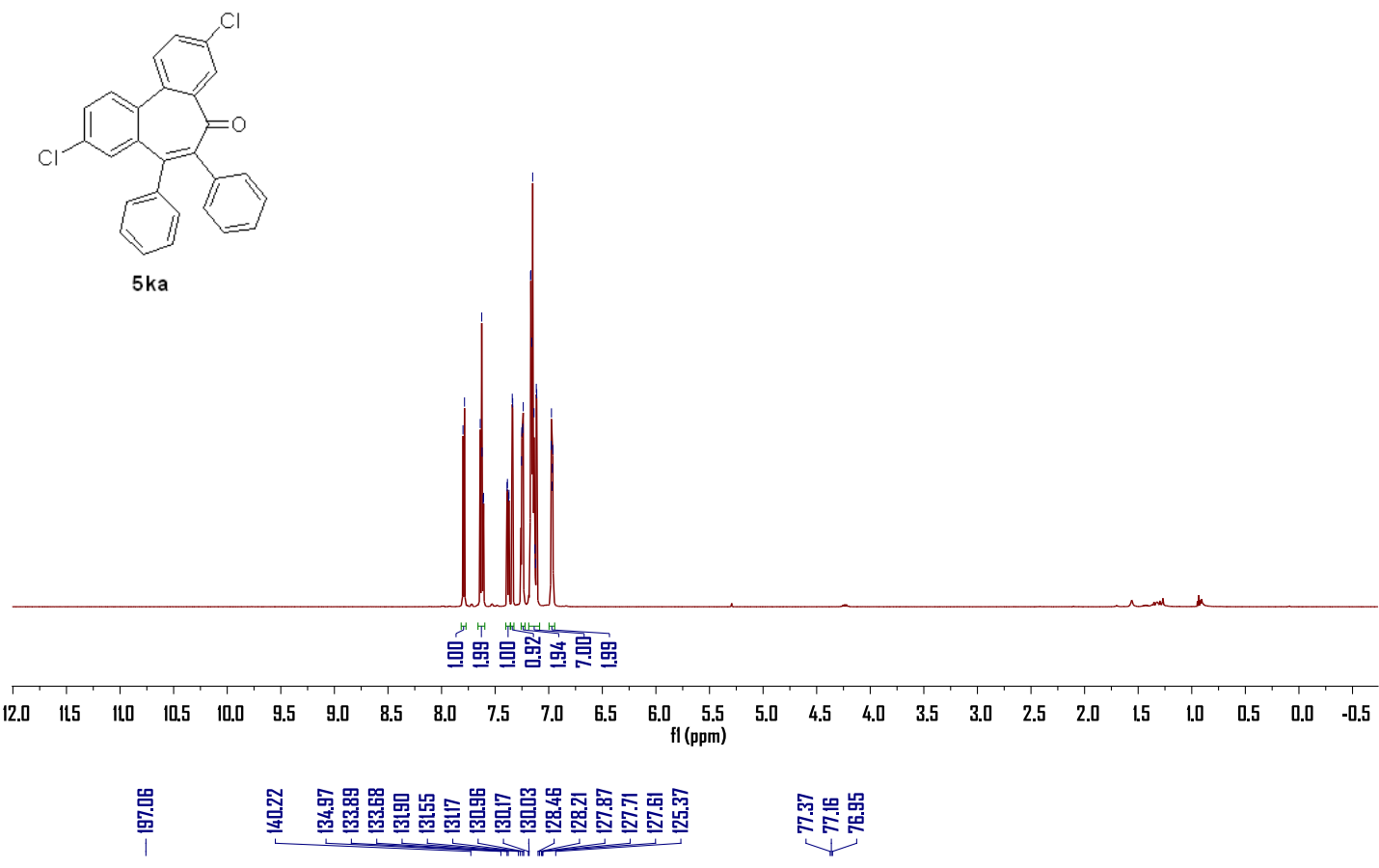

${ }^{13} \mathrm{C} \mathrm{NMR}\left(150 \mathrm{MHz}, \mathrm{CDCl}_{3}\right)$

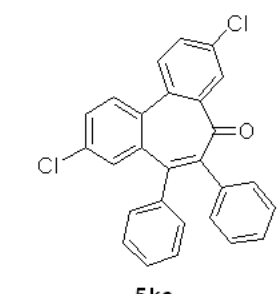

$5 \mathrm{ka}$

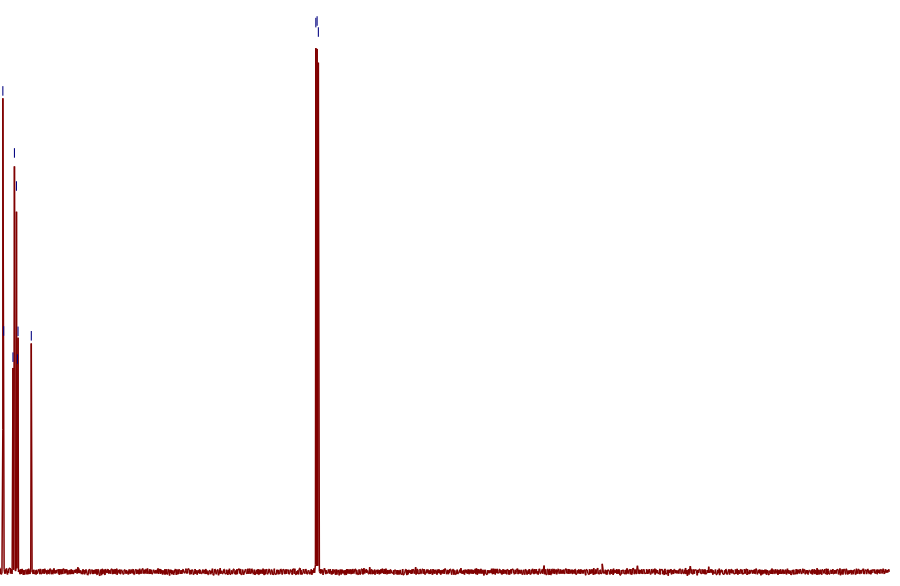

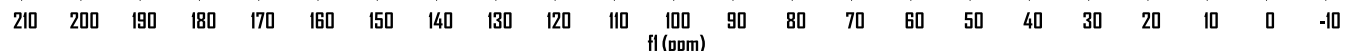




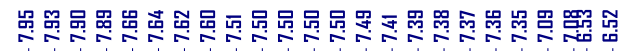

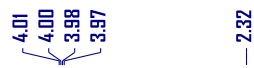

${ }^{1} \mathrm{HNMR}\left(600 \mathrm{MHz}, \mathrm{CDCl}_{3}\right)$

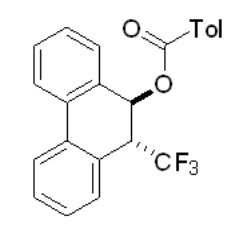

6

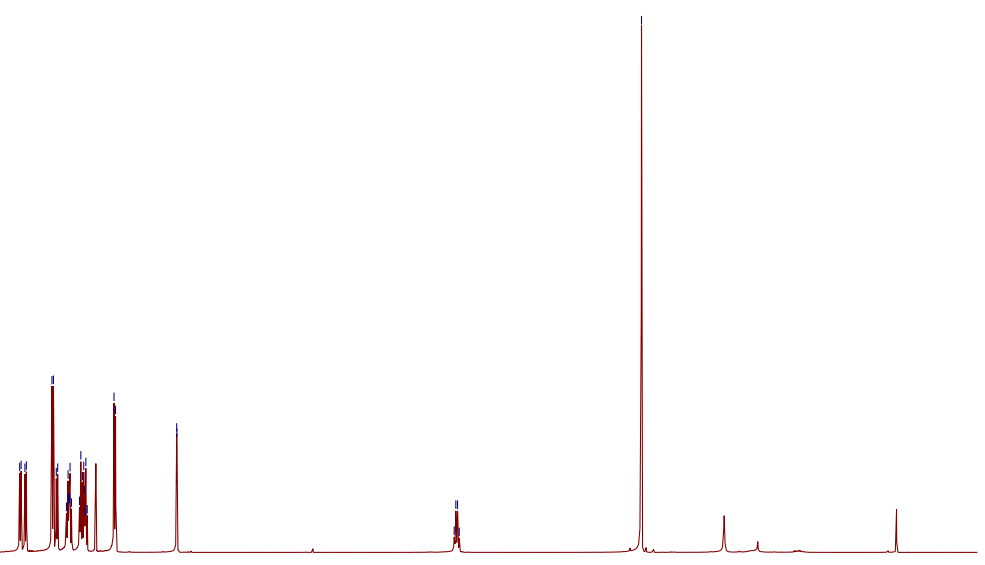

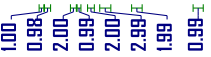

亘葛

$\begin{array}{lllllllllllllllllllllllllllllll}1 & 12.0 & 11.5 & 11.0 & 10.5 & 10.0 & 9.5 & 9.0 & 8.5 & 8.0 & 7.5 & 7.0 & 6.5 & \begin{array}{c}6.0 \\ \mathrm{f}(\mathrm{ppm})\end{array} & 5.0 & 4.5 & 4.0 & 3.5 & 3.0 & 2.5 & 2.0 & 1.5 & 1.0 & 0.5 & 0.0 & -0.5\end{array}$

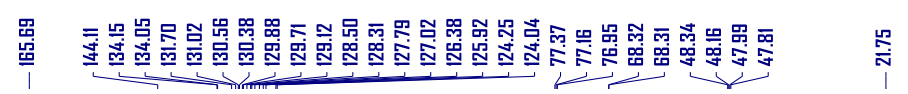

${ }^{13} \mathrm{C} \mathrm{NMR}\left(150 \mathrm{MHz}, \mathrm{CDCl}_{3}\right)$

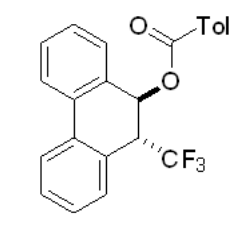

6

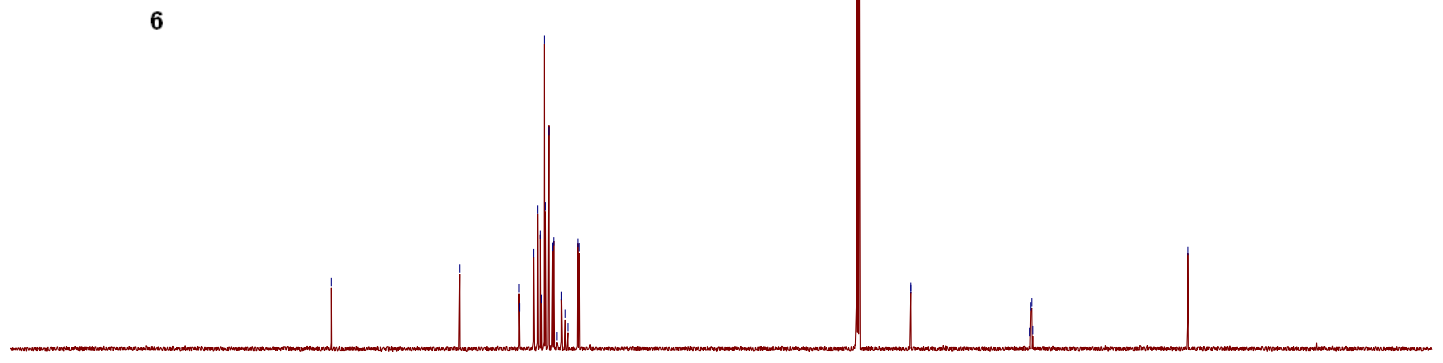

$\begin{array}{lllllllllllllllllllllll}210 & 200 & 190 & 180 & 170 & 160 & 150 & 140 & 130 & 120 & 110 & 100 & 90 & 80 & 70 & 60 & 50 & 40 & 30 & 20 & 10 & 0 & -10\end{array}$ 
${ }^{19} \mathrm{~F} \mathrm{NMR}\left(565 \mathrm{MHz}, \mathrm{CDCl}_{3}\right)$

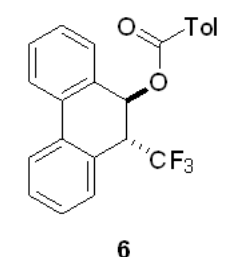

6

$\begin{array}{lllllllllllllllllllllllllllllllllllllllll}10 & 0 & -10 & -20 & -30 & -40 & -50 & -60 & -70 & -80 & -90 & -100 & -110 & -120 & -130 & -140 & -150 & -160 & -170 & -180 & -190 & -200 & -210\end{array}$

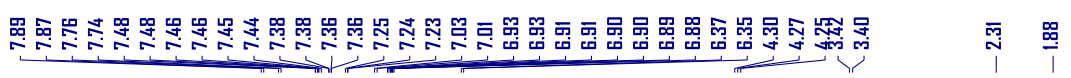

${ }^{1} \mathrm{H} \mathrm{NMR}\left(400 \mathrm{MHz}, \mathrm{CDCl}_{3}\right)$
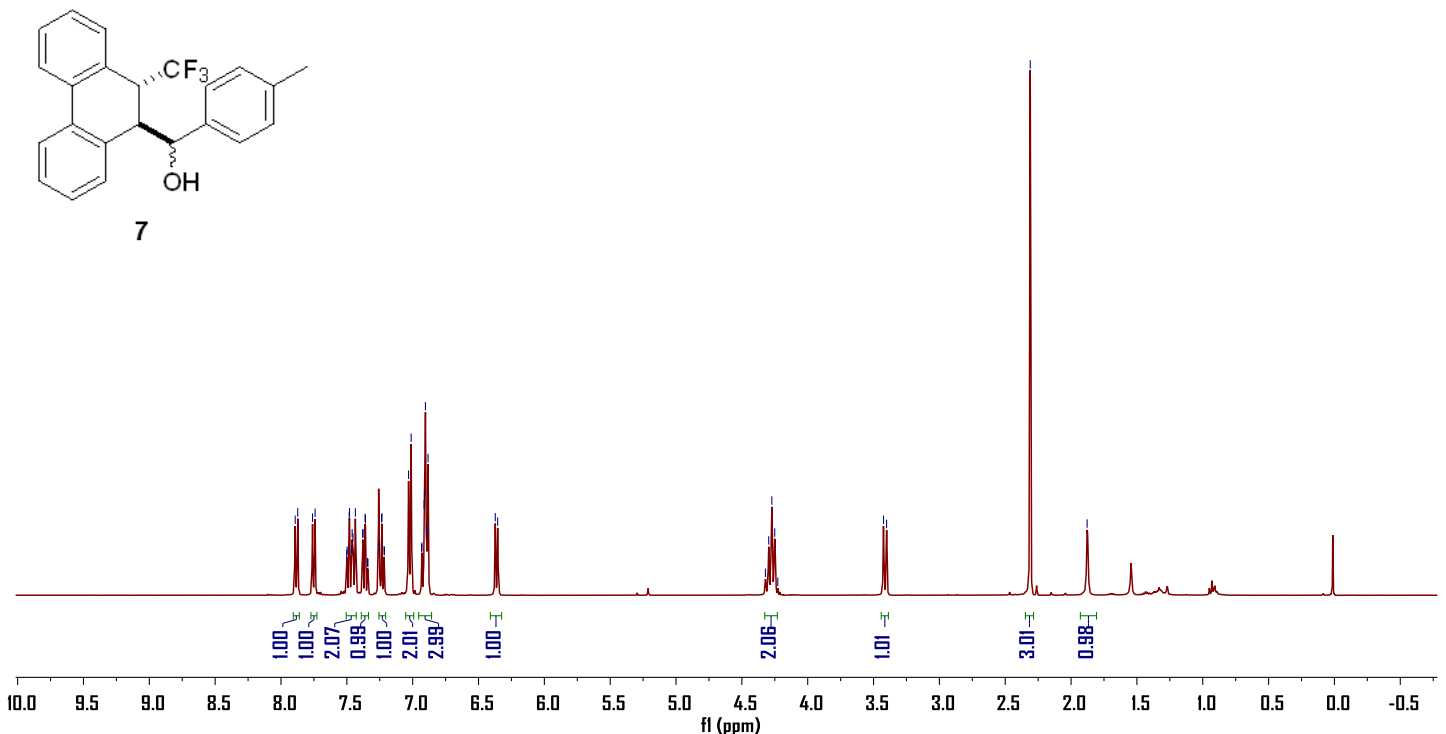


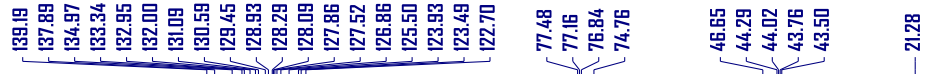

${ }^{13} \mathrm{C} \mathrm{NMR}\left(100 \mathrm{MHz}, \mathrm{CDCl}_{3}\right)$
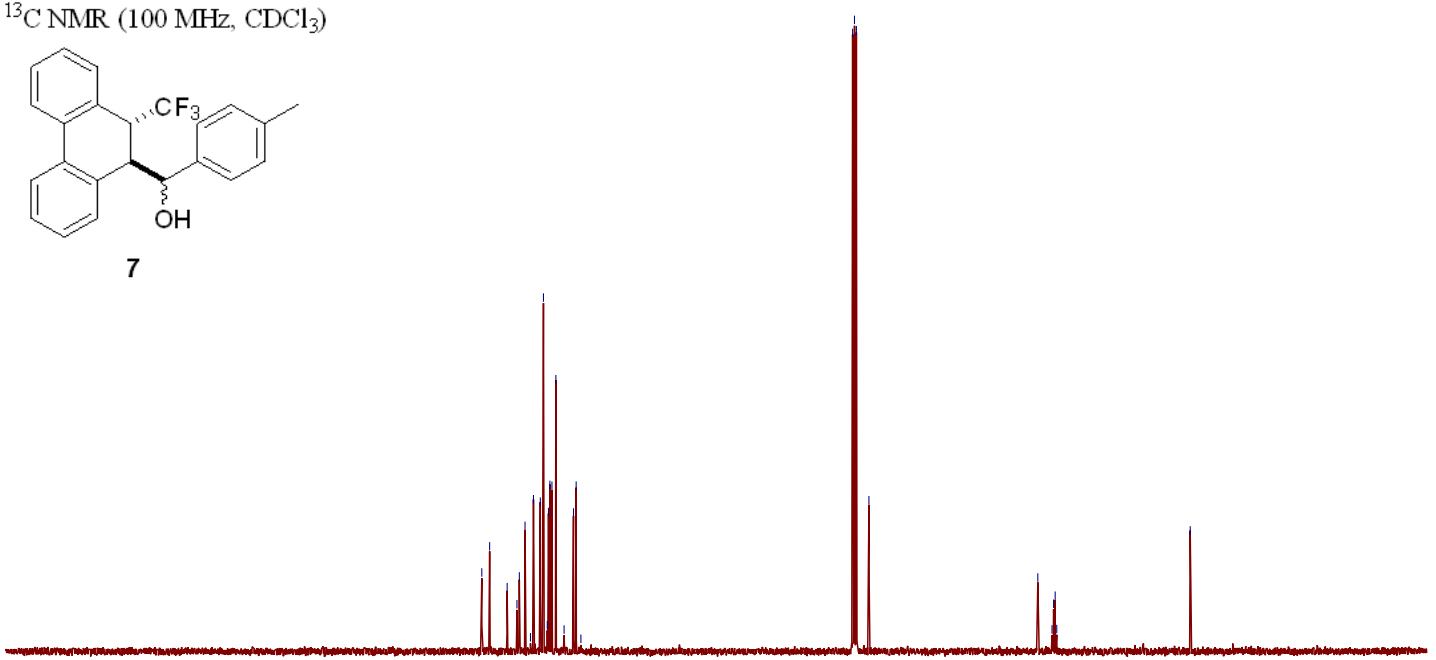

$\begin{array}{llllllllllllllllllllllll}210 & 200 & 190 & 180 & 170 & 160 & 150 & 140 & 130 & 120 & 110 & \begin{array}{l}100 \\ \mathrm{fl}(\mathrm{pmm})\end{array} & 90 & 80 & 70 & 60 & 50 & 40 & 30 & 20 & 10 & 0 & -10\end{array}$

\section{㚿骂}

${ }^{19} \mathrm{~F} \mathrm{NMR}\left(376 \mathrm{MHz}, \mathrm{CDCl}_{3}\right)$

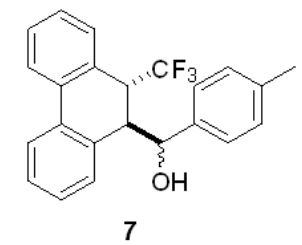

7

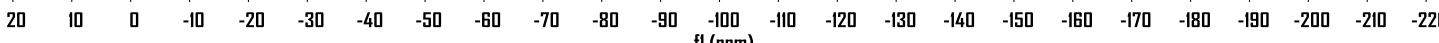


${ }^{1} \mathrm{H} \mathrm{NMR}\left(400 \mathrm{MHz}, \mathrm{CDCl}_{3}\right)$
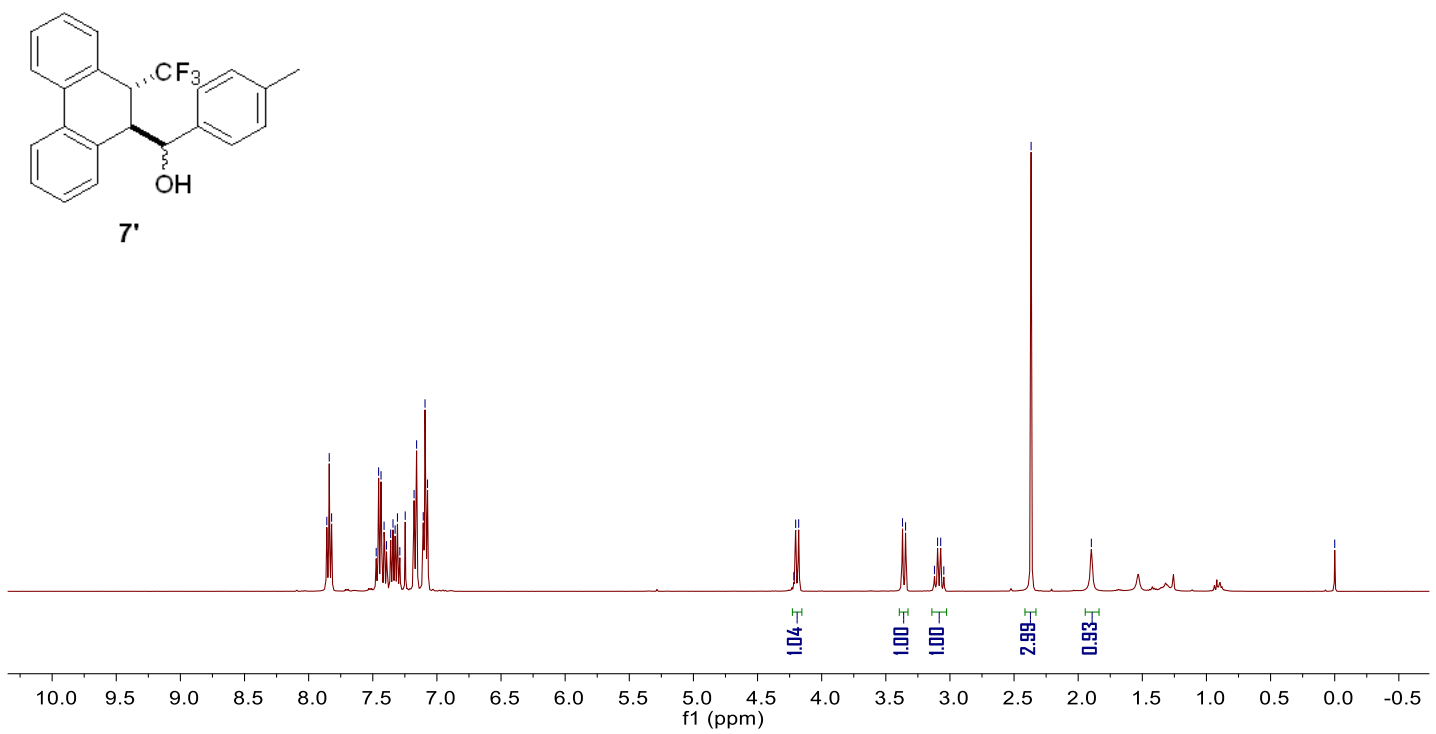

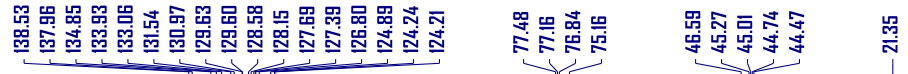

${ }^{13} \mathrm{C} \mathrm{NMR}\left(100 \mathrm{MHz}, \mathrm{CDCl}_{3}\right)$
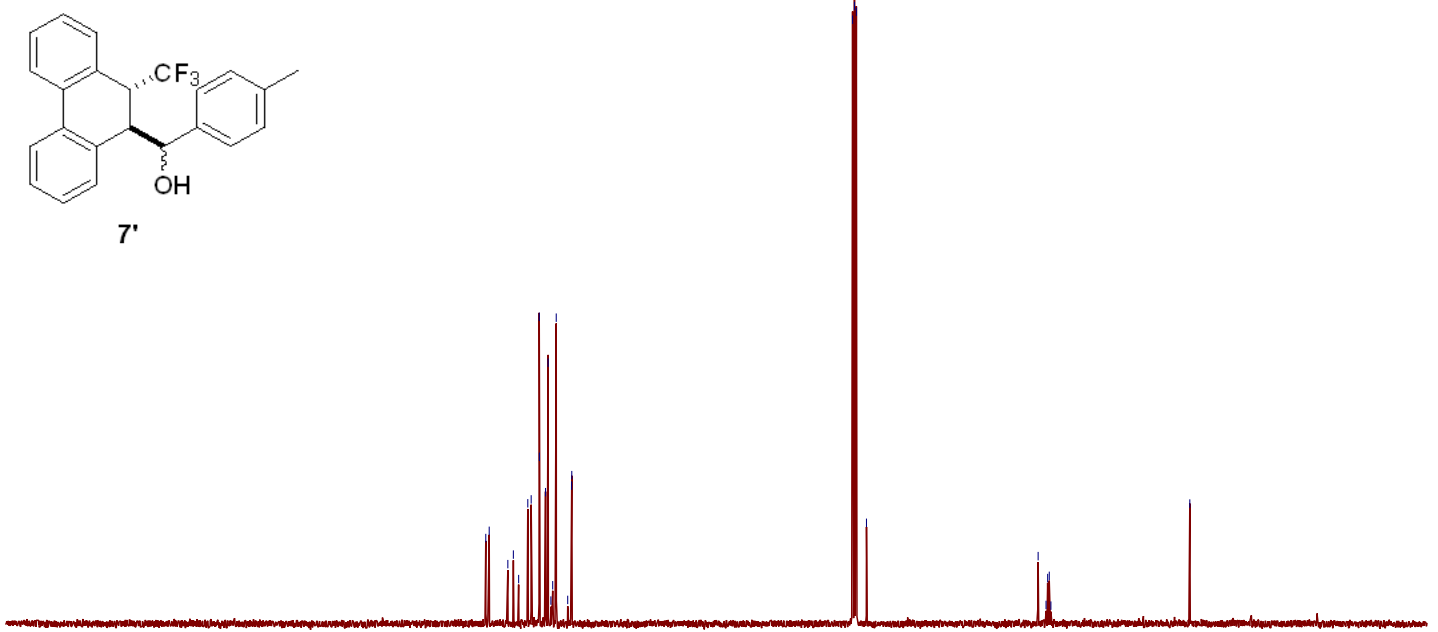

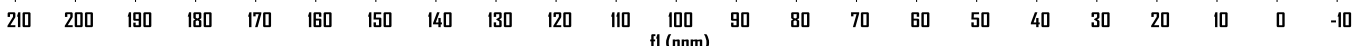


${ }^{19} \mathrm{~F} \mathrm{NMR}\left(376 \mathrm{MHz}, \mathrm{CDCl}_{3}\right)$<smiles>Cc1ccc(C(O)C2c3ccccc3-c3ccccc3[C@@H]2F)cc1</smiles>

7'

\begin{tabular}{llllllllllllllllllllllllllll}
\hline 20 & 10 & 0 & -10 & -20 & -30 & -40 & -50 & -60 & -70 & -80 & -90 & -100 & -110 & -120 & -130 & -140 & -150 & -160 & -170 & -180 & -190 & -200 & -210 & -220
\end{tabular}

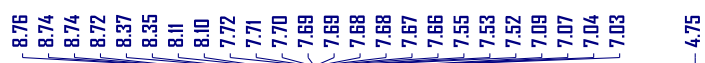

${ }^{1} \mathrm{HNMR}\left(600 \mathrm{MHz}, \mathrm{CDCl}_{3}\right)$
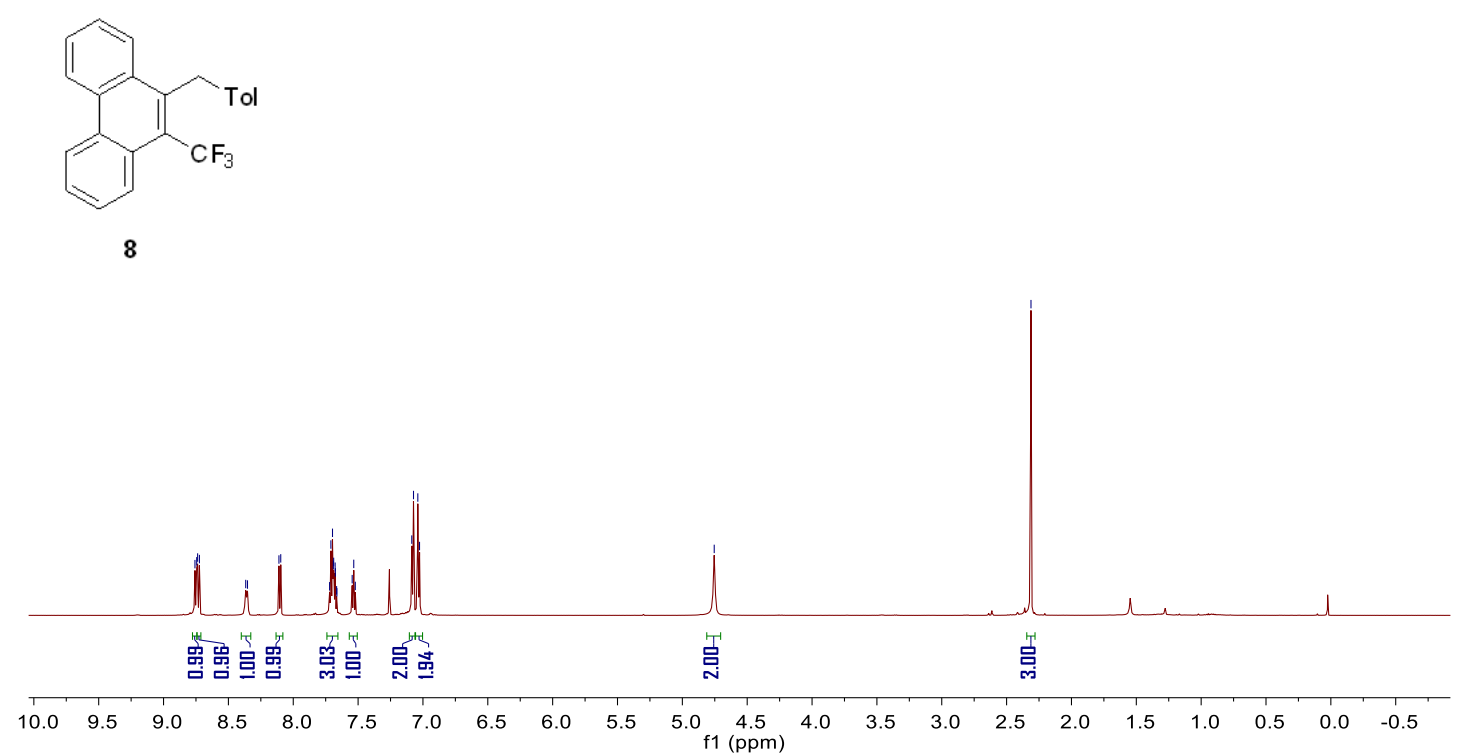


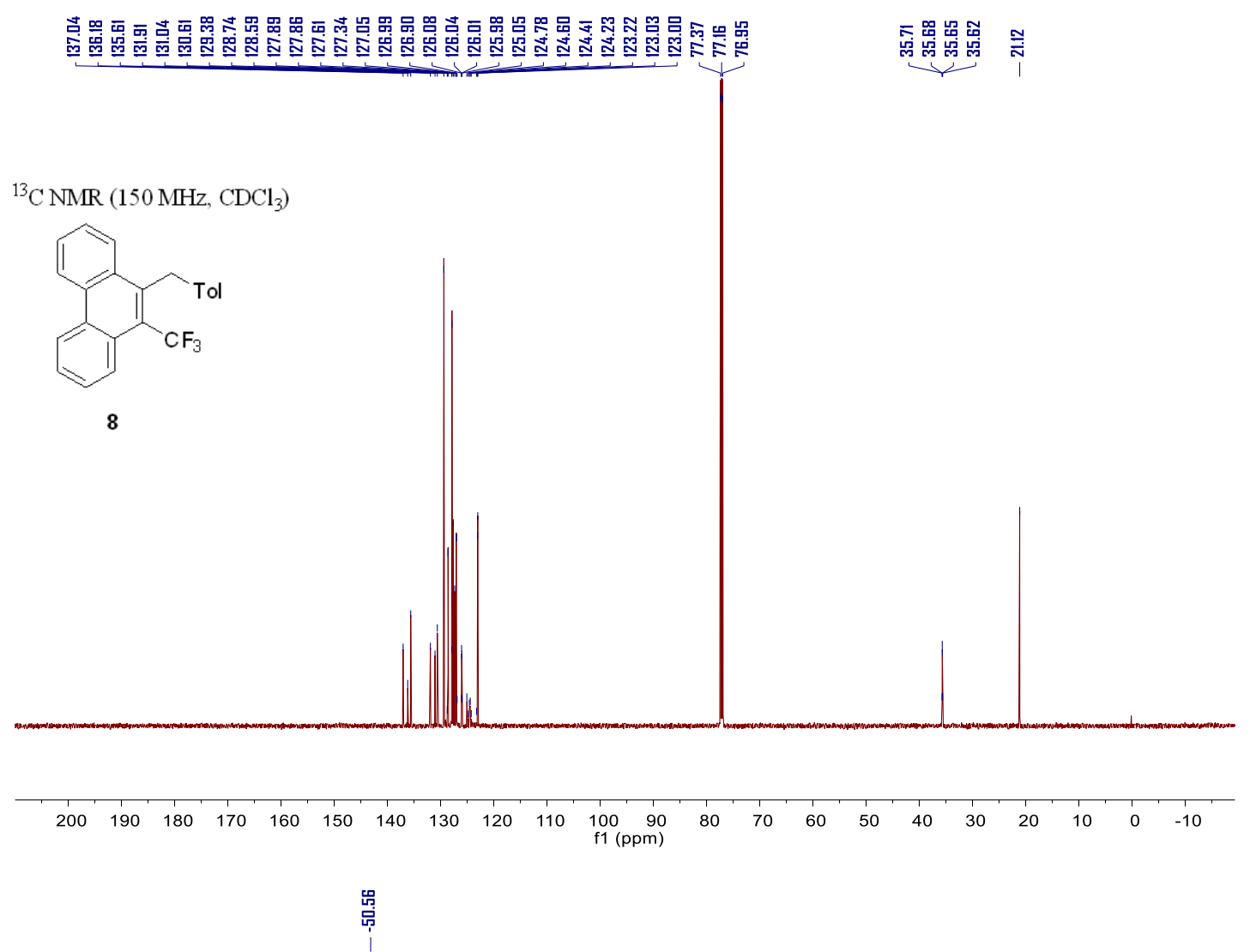

${ }^{19} \mathrm{~F} \mathrm{NMR}\left(565 \mathrm{MHz}, \mathrm{CDCl}_{3}\right)$

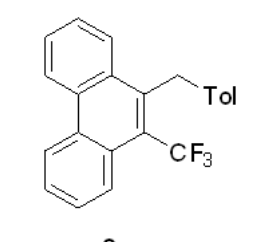

8

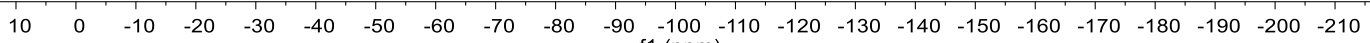




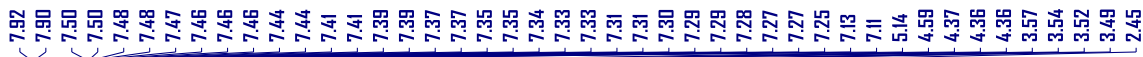

${ }^{1} \mathrm{HNMR}\left(400 \mathrm{MHz}, \mathrm{CDCl}_{3}\right)$<smiles>C=C(Cl)C1c2ccccc2-c2ccccc2[C@H]1C(F)(F)F</smiles>

9

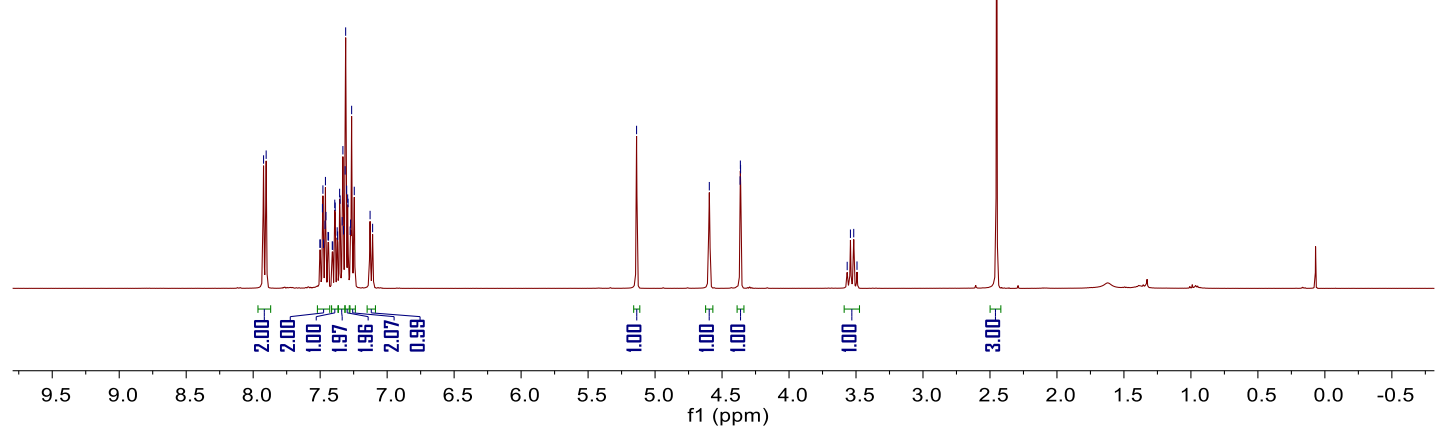

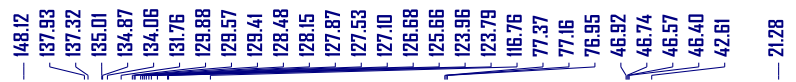

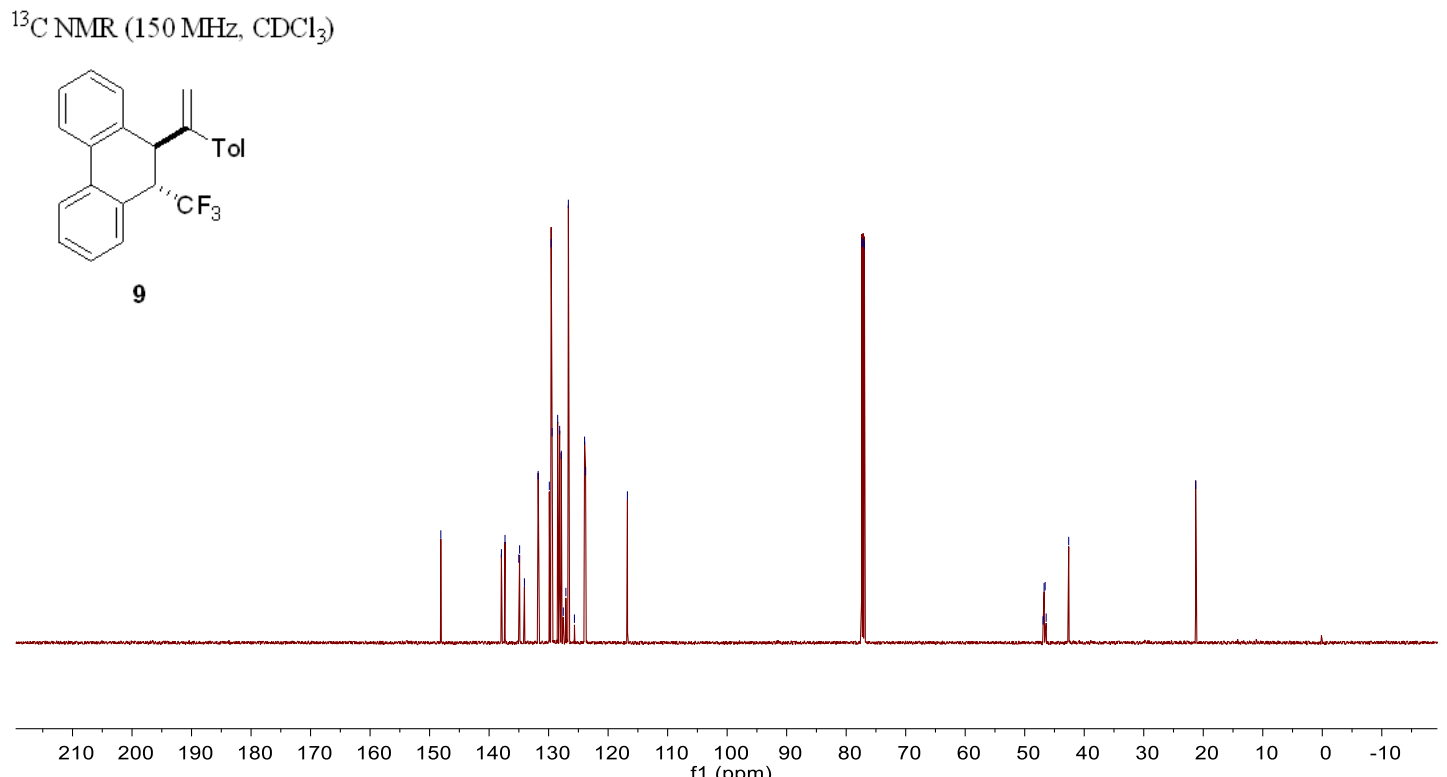


${ }^{19} \mathrm{~F} \mathrm{NMR}\left(376 \mathrm{MHz}, \mathrm{CDCl}_{3}\right)$<smiles>C=C(Cl)C1c2ccccc2-c2ccccc2C1C(F)(F)F</smiles>

9

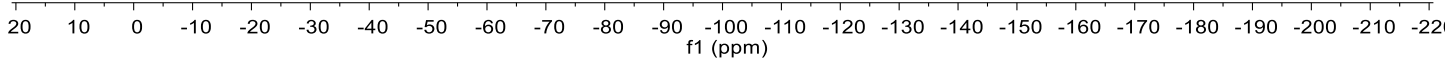

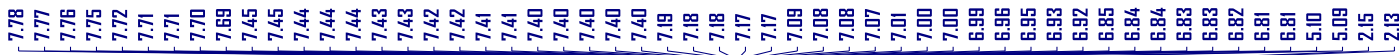

${ }^{1} \mathrm{HNMR}\left(400 \mathrm{MHz}, \mathrm{CDCl}_{3}\right)$

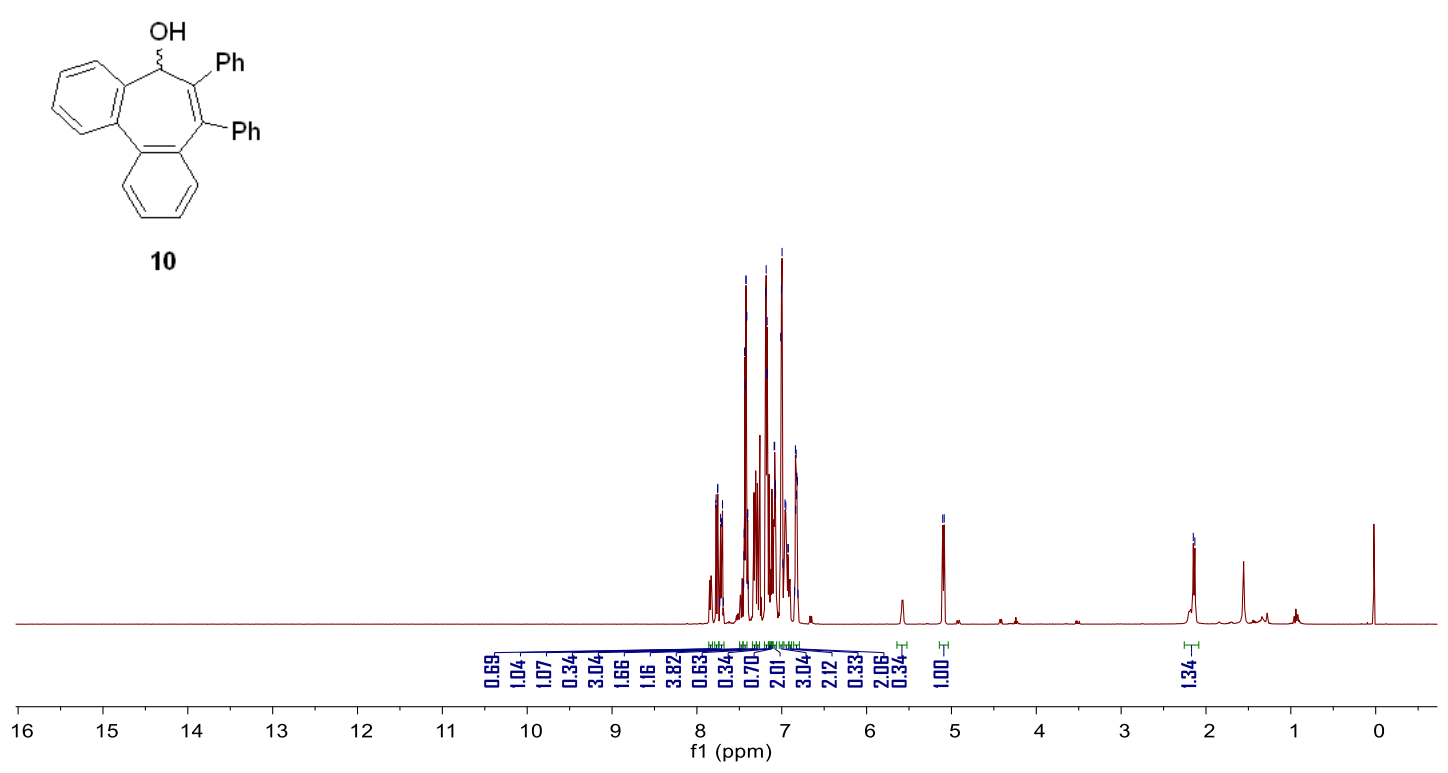




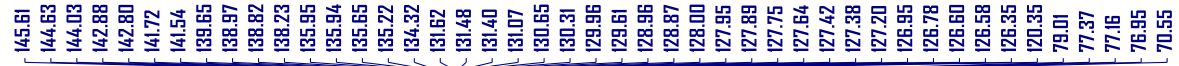

${ }^{13} \mathrm{C} \mathrm{NMR}\left(150 \mathrm{MHz}, \mathrm{CDCl}_{3}\right)$

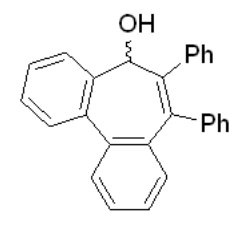

10

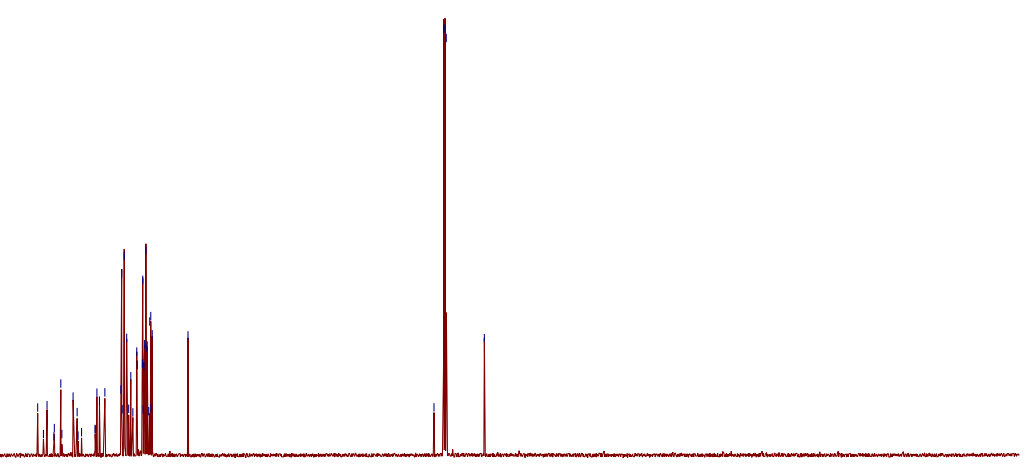

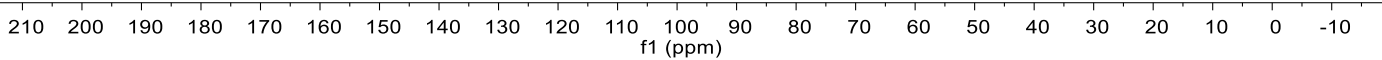

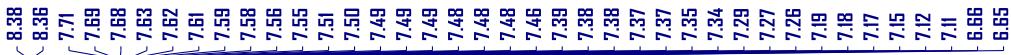

${ }^{1} \mathrm{H} \mathrm{NMR}\left(600 \mathrm{MHz}, \mathrm{CDCl}_{3}\right)$

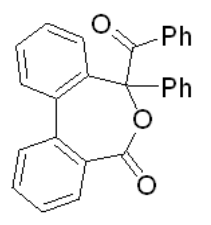

11

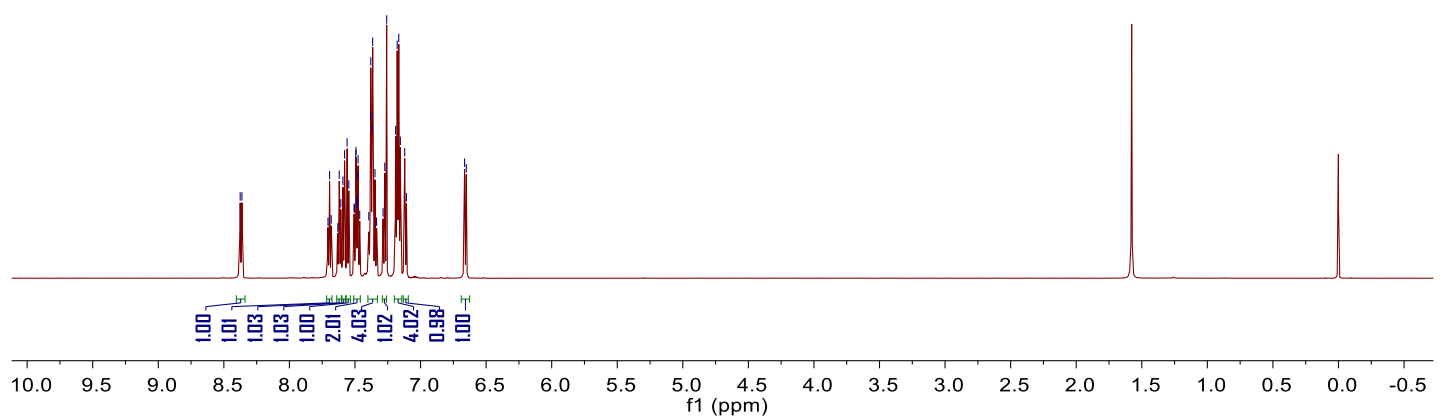




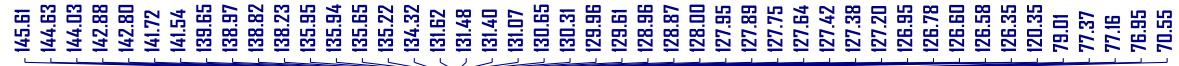

${ }^{13} \mathrm{CNMR}\left(150 \mathrm{MHz}, \mathrm{CDCl}_{3}\right)$

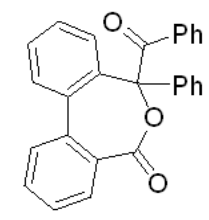

11

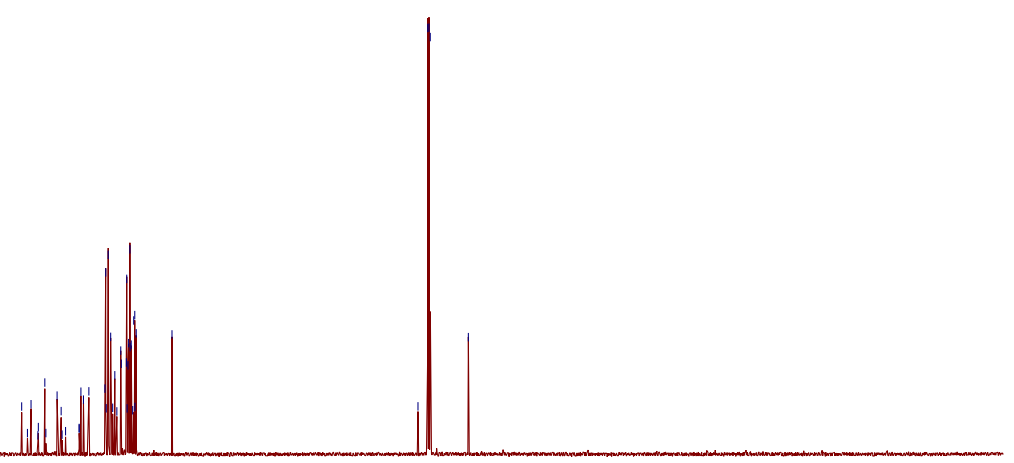

$\begin{array}{lllllllllllllllllllllll}210 & 200 & 190 & 180 & 170 & 160 & 150 & 140 & 130 & 120 & 110 & 100 & 90 & 80 & 70 & 60 & 50 & 40 & 30 & 20 & 10 & 0 & -10\end{array}$

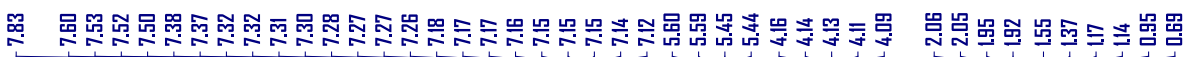

${ }^{1} \mathrm{HNMR}\left(600 \mathrm{MHz}, \mathrm{CDCl}_{3}\right)$

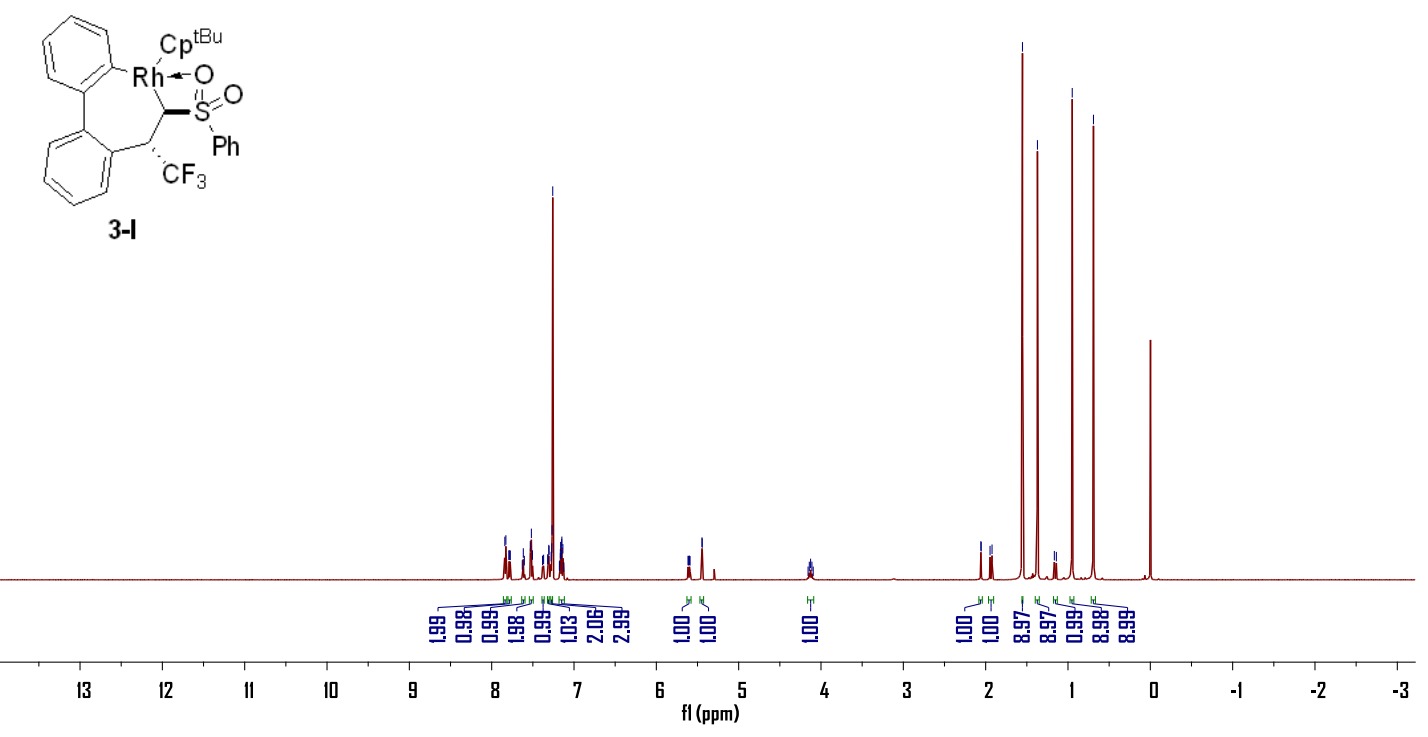



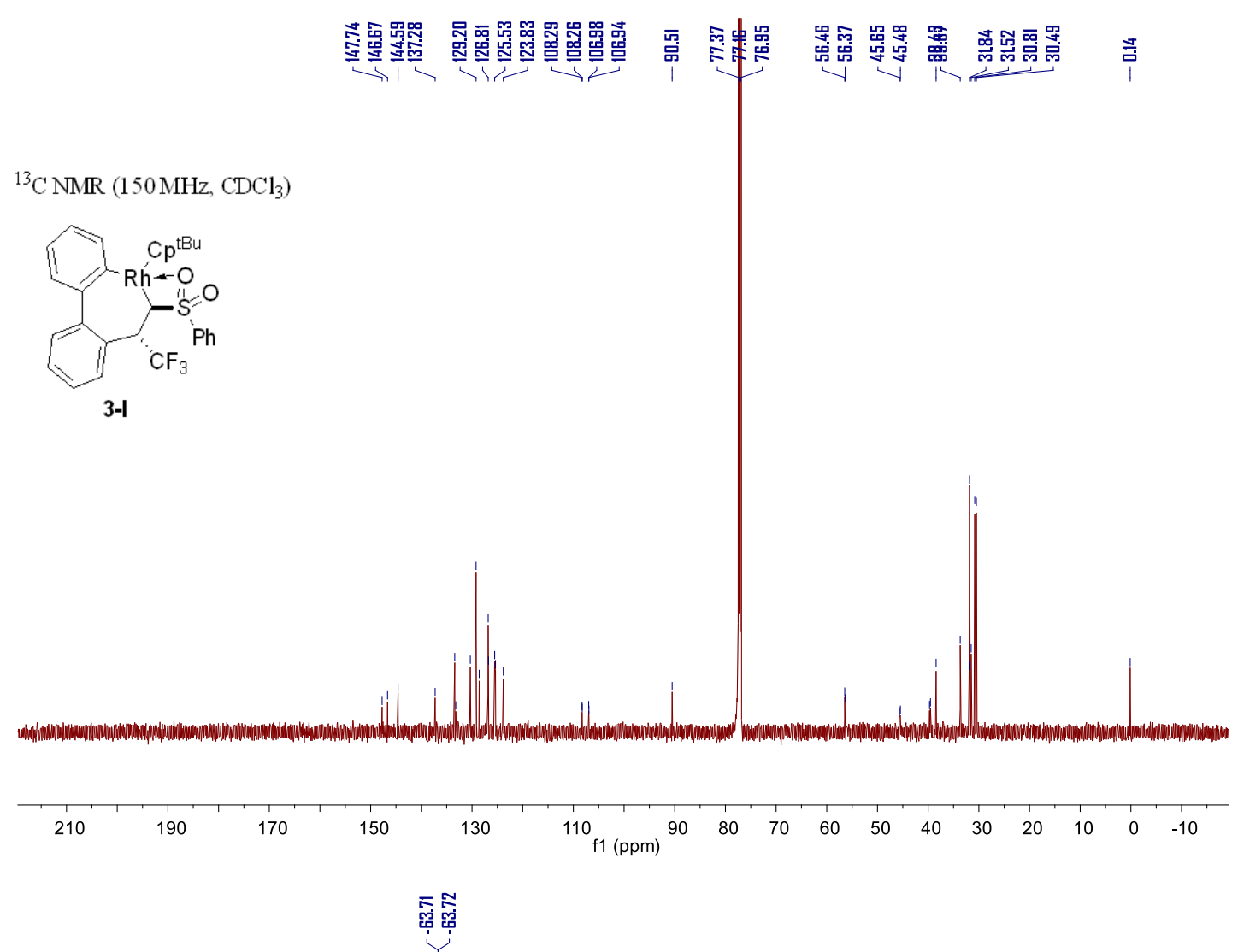

${ }^{19} \mathrm{FNMR}\left(565 \mathrm{MHz}, \mathrm{CDCl}_{3}\right)$

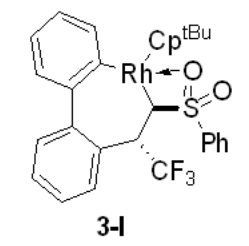

3-I

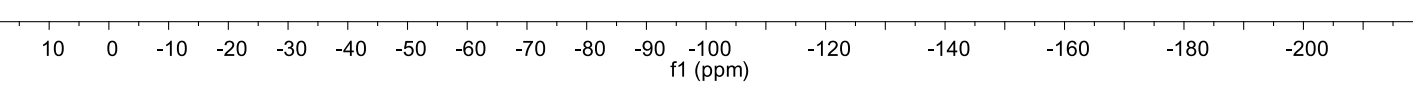




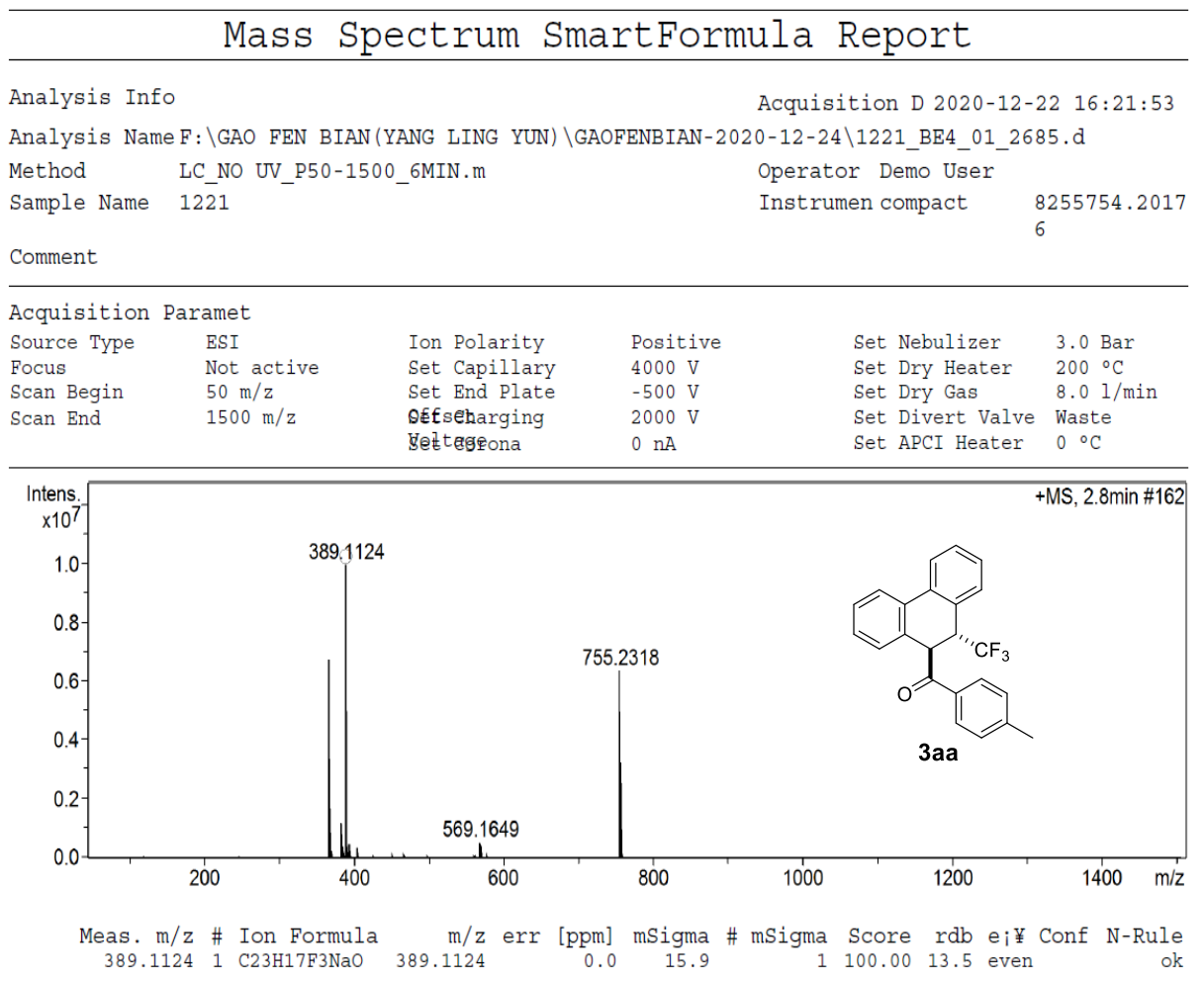

\section{Mass Spectrum SmartFormula Report}

Analysis Info

Acquisition D 2021-05-16 11:39:28

Analysis Name F:\GAO FEN BIAN(YANG LING YUN) \GAOFENBIAN-2021-05-14\0514_RC5_01_6019.d

Method LC_NO UV_P50-1500_20MIN.m Operator Demo User

Sample Name $0514 \quad$ Instrumen compact

8255754.2017

Comment

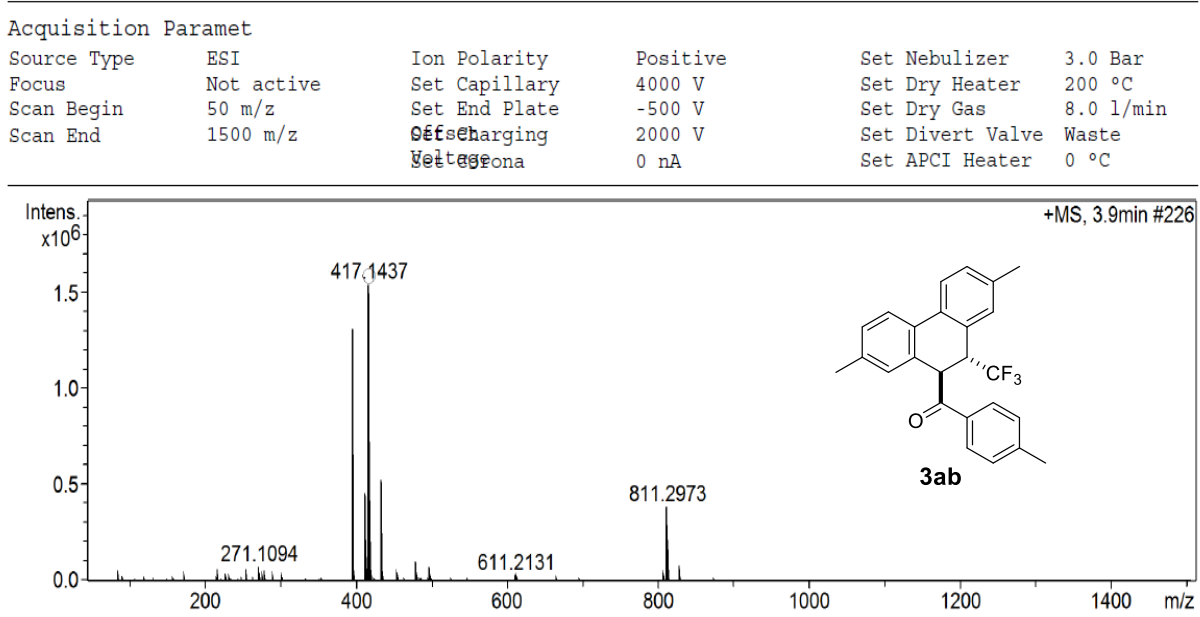

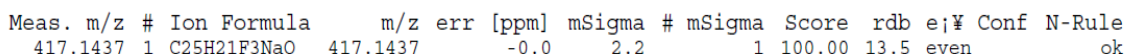
417.1437

$\begin{array}{rrr}-0.0 & 2.2\end{array}$

1100.0013 .5 even 


\section{Mass Spectrum SmartFormula Report}

Analysis Info

Acquisition D 2021-01-28 4:52:09

Analysis Name F:\GAO FEN BIAN(YANG LING YUN) \GAOFENBIAN-2021-1-29\0125_BB4_01_4029.d

Method LC_NO UV_P50-1500_6MIN.m Operator Demo User

Sample Name $0125 \quad$ Instrumen compact

8255754.2017

Comment

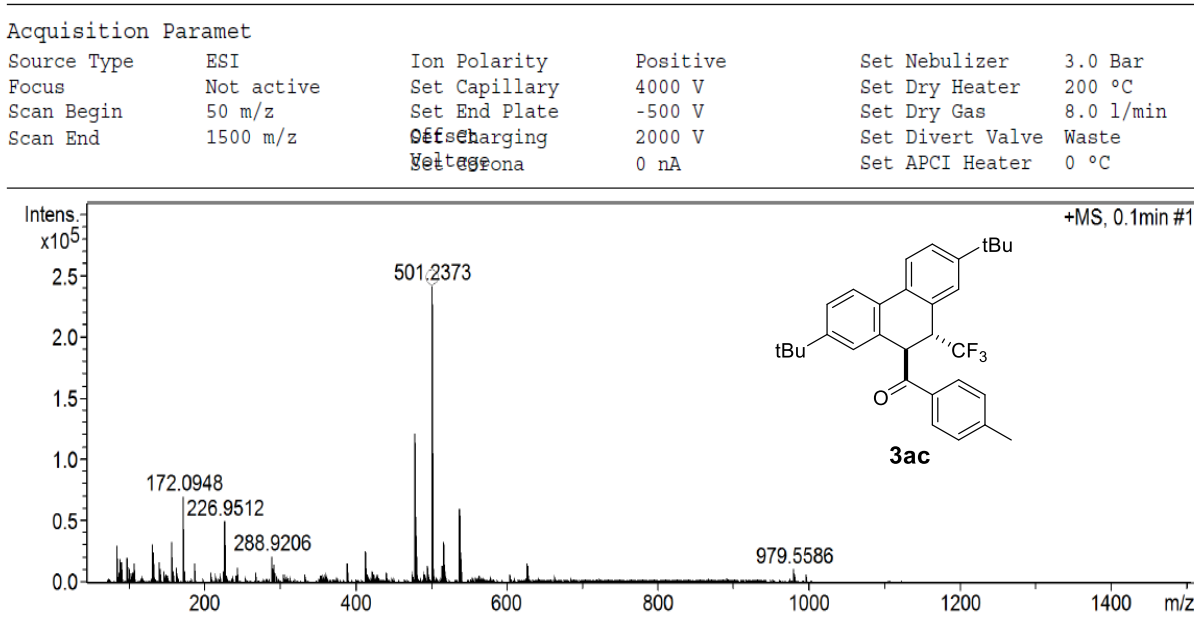

Meas. $\mathrm{m} / \mathrm{z}$ \# Ion Formula $\mathrm{m} / \mathrm{z}$ err [ppm] mSigma \# mSigma Score $\mathrm{rdb} e_{i} ¥$ Conf $\mathrm{N}-\mathrm{Rule}$

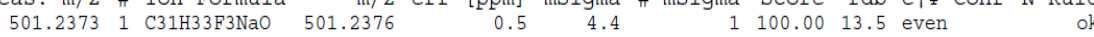

\section{Mass Spectrum SmartFormula Report}

Analysis Info

Acquisition D 2021-05-16 9:51:48

Analysis Name F: \GAO FEN BIAN(YANG LING YUN) \GAOFENBIAN-2021-05-14\0514_RB8_01_6014.d

Method LC_NO UV_P50-1500_20MIN.m Operator Demo User

Sample Name 0514

Instrumen compact

8255754.2017

Comment

Acquisition Paramet

$\begin{array}{llllll}\text { Source Type } & \text { ESI } & \text { Ion Polarity } & \text { Positive } & \text { Set Nebulizer } & 3.0 \text { Bar } \\ \text { Focus } & \text { Not active } & \text { Set Capillary } & 4000 \mathrm{~V} & \text { Set Dry Heater } & 200^{\circ} \mathrm{C} \\ \text { Scan Begin } & 50 \mathrm{~m} / \mathrm{z} & \text { Set End Plate } & -500 \mathrm{~V} & \text { Set Dry Gas } & 8.0 \mathrm{l} / \mathrm{min} \\ \text { Scan End } & 1500 \mathrm{~m} / \mathrm{z} & \text { OEf } & \text { Setharging } & 2000 \mathrm{~V} & \text { Set Divert Valve Waste } \\ & & 0 \mathrm{nA} & \text { Set APCI Heater } 0{ }^{\circ} \mathrm{C}\end{array}$

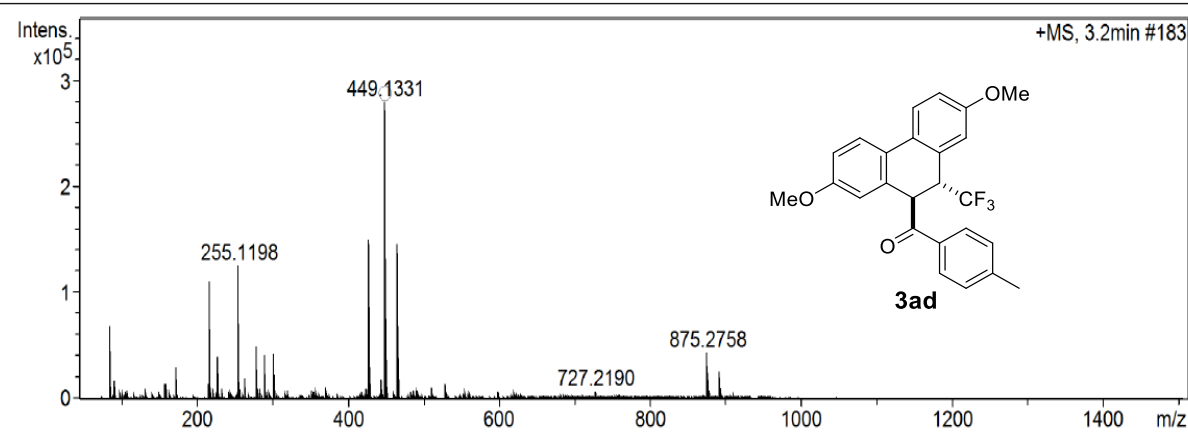

Meas. m/z \# Ion Formula $\mathrm{m} / \mathrm{z}$ err [ppm] mSigma \# mSigma Score rdb e $¥ ¥$ Conf $\mathrm{N}-$ Rule

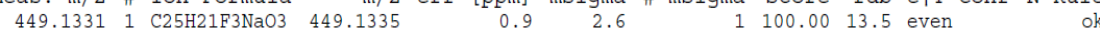


Analysis Info

Acquisition D 2020-12-22 16:52:21 Analysis Name F:\GAO FEN BIAN(YANG LING YUN) \GAOFENBIAN-2020-12-24\1221_BE8_01_2689.d

Method LC_NO UV_P50-1500_6MIN.m Operator Demo User

Sample Name $12 \overline{2} 1$

Instrumen compact

8255754.2017

Comment

\begin{tabular}{llllll}
\hline Acquisition & Paramet & & & & \\
Source Type & ESI & Ion Polarity & Positive & Set Nebulizer & $3.0 \mathrm{Bar}$ \\
Focus & Not active & Set Capillary & $4000 \mathrm{~V}$ & Set Dry Heater & $200^{\circ} \mathrm{C}$ \\
Scan Begin & $50 \mathrm{~m} / \mathrm{z}$ & Set End Plate & $-500 \mathrm{~V}$ & Set Dry Gas & $8.0 \mathrm{l} / \mathrm{min}$ \\
Scan End & $1500 \mathrm{~m} / \mathrm{z}$ & 96fseharging & $2000 \mathrm{~V}$ & Set Divert Valve Waste \\
& & Settegena & $0 \mathrm{nA}$ & Set APCI Heater $0{ }^{\circ} \mathrm{C}$
\end{tabular}

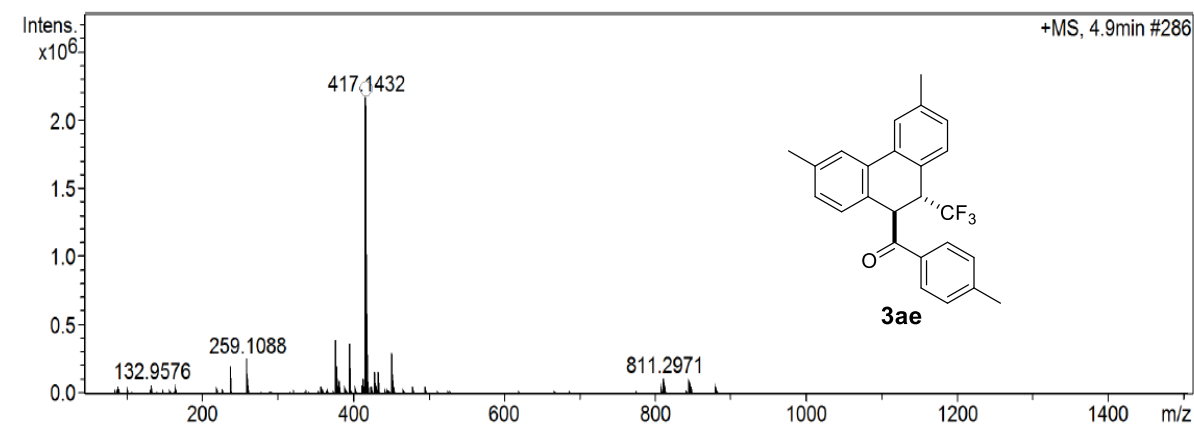

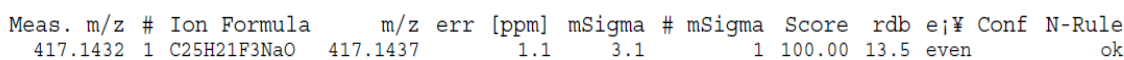

1100.0013 .5 even

\section{Mass Spectrum SmartFormula Report}

Analysis Info

Acquisition D 2021-01-28 7:57:39

Analysis Name F: \GAOFENBIAN-2021-1-29\0125_BE5_01_4054.d

Method LC_NO UV_P50-1500_6MIN.m

Operator Demo User

Sample Name $01 \overline{2} 5$

Instrumen compact

8255754.2017

Comment

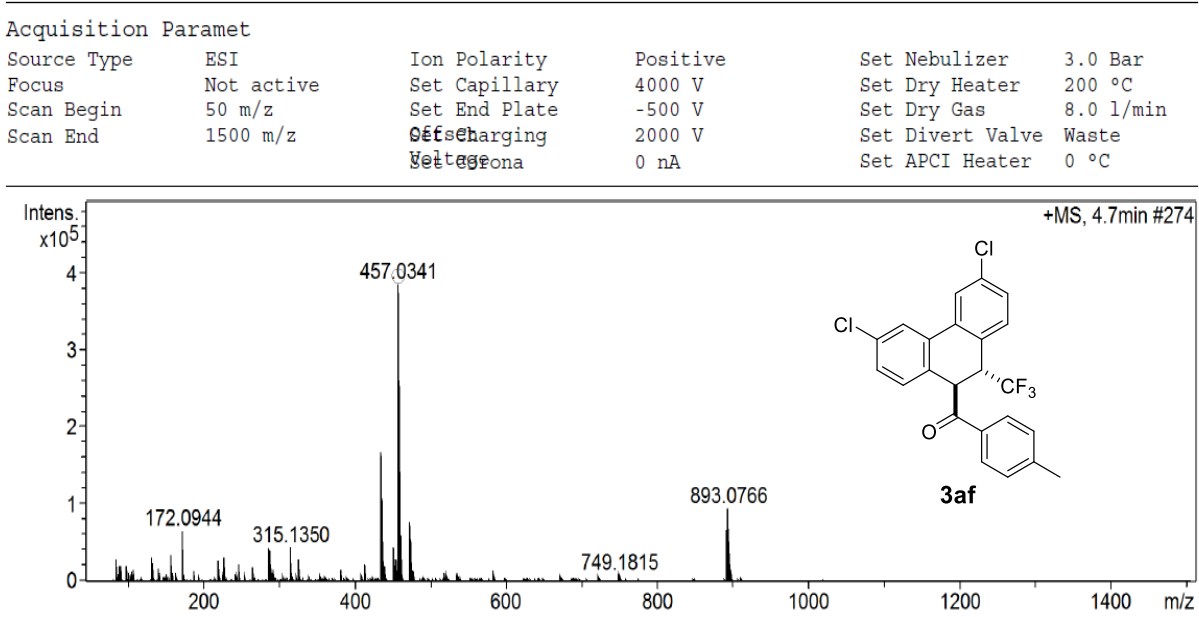

Meas. $\mathrm{m} / \mathrm{z}$ \# Ion Formula $\mathrm{m} / \mathrm{z}$ err [ppm] mSigma \# mSigma Score rdb ej¥ Conf $\mathrm{N}-\mathrm{Rul}$

457.0341 1 $233 \mathrm{H} 15 \mathrm{Cl} 2 \mathrm{~F} 3 \mathrm{NaO} \quad 457.0344 \quad 0.8 \quad 3.0=13.5$ even 


\section{Mass Spectrum SmartFormula Report}

Analysis Info

Acquisition D 2021-01-28 5:07:21

Analysis Name F:\GAO FEN BIAN(YANG LING YUN) \GAOFENBIAN-2021-1-29\0125_BB6_01_4031.d

Method LC_NO UV_P50-1500_6MIN.m Operator Demo User

Sample Name 0125

Instrumen compact

8255754.2017

Comment

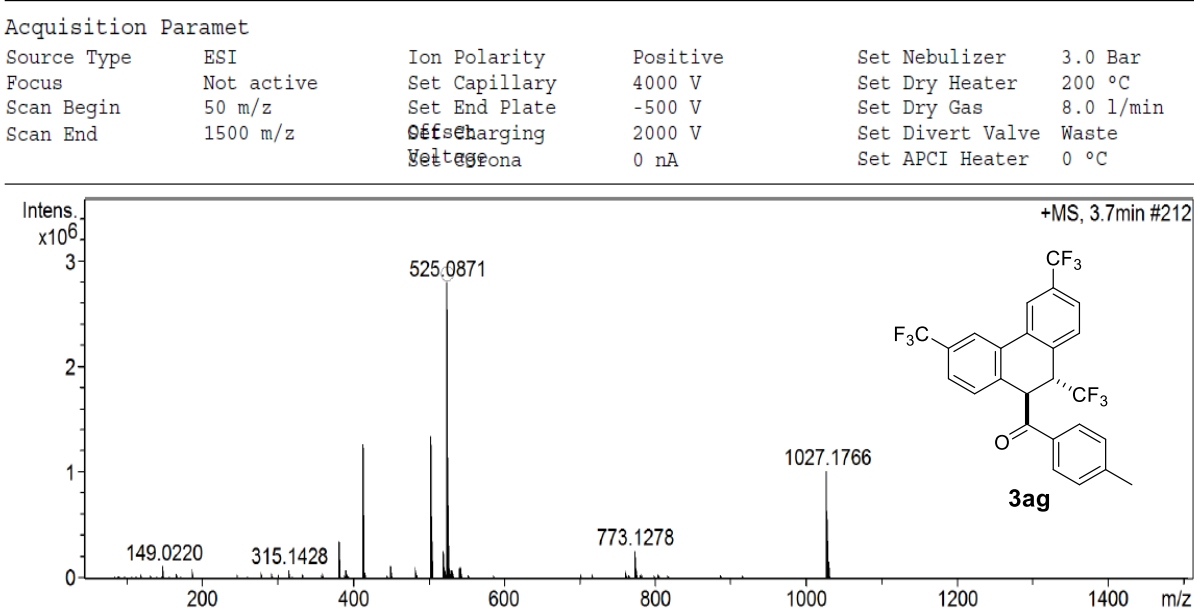

Meas. $\mathrm{m} / \mathrm{z}$ \# Ion Formula $\mathrm{m} / \mathrm{z}$ err $[\mathrm{ppm}]$ mSigma \# mSigma Score $\mathrm{rdb} e_{i} ¥$ Conf $\mathrm{N}-\mathrm{Rule}$

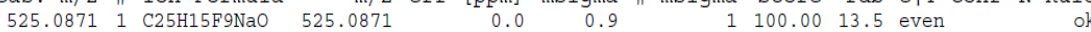

\section{Mass Spectrum SmartFormula Report}

Analysis Info

Acquisition D 2020-12-22 16:37:37

Analysis Name $\mathrm{F}: \backslash$ GAO FEN BIAN(YANG LING YUN) \GAOFENBIAN-2020-12-24\1221_BE6_01_2687.d

Method

LC_NO UV_P50-1500_6MIN.m

operator Demo User

Sample Name 1221

Instrumen compact

8255754.2017

Comment

Acquisition Paramet

$\begin{array}{llllll}\text { Source Type } & \text { ESI } & \text { Ion Polarity } & \text { Positive } & \text { Set Nebulizer } & 3.0 \text { Bar } \\ \text { Focus } & \text { Not active } & \text { Set Capillary } & 4000 \mathrm{~V} & \text { Set Dry Heater } & 200^{\circ} \mathrm{C} \\ \text { Scan Begin } & 50 \mathrm{~m} / \mathrm{z} & \text { Set End Plate } & -500 \mathrm{~V} & \text { Set Dry Gas } & 8.0 \mathrm{l} / \mathrm{min} \\ \text { Scan End } & 1500 \mathrm{~m} / \mathrm{z} & \text { Offsebarging } & 2000 \mathrm{~V} & \text { Set Divert Valve Waste } \\ & & \text { Settagena } & 0 \mathrm{nA} & \text { Set APCI Heater } 0{ }^{\circ} \mathrm{C}\end{array}$

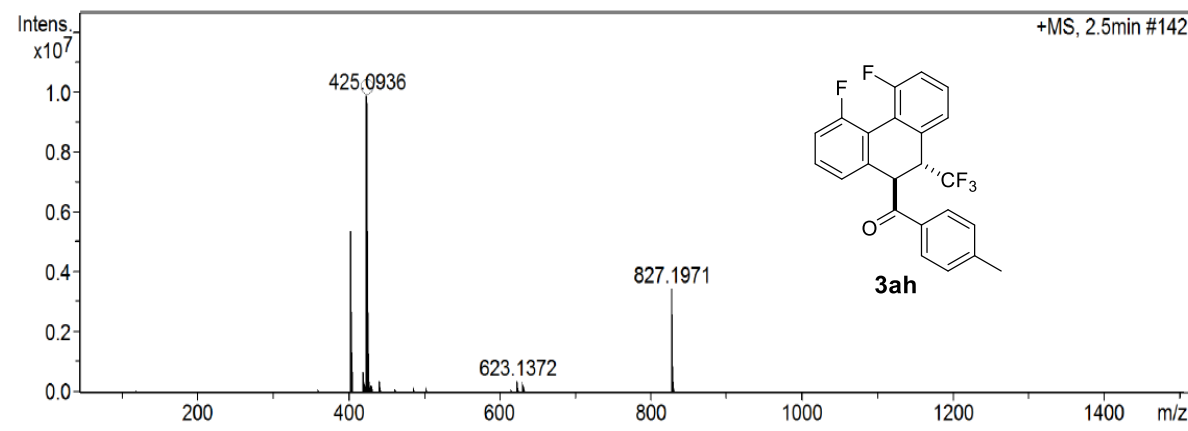

Meas. $\mathrm{m} / \mathrm{z}$ \# Ion Formula $\mathrm{m} / \mathrm{z}$ err [ppm] mSigma \# mSigma Score rdb e i i Conf $\mathrm{N}$-Rule

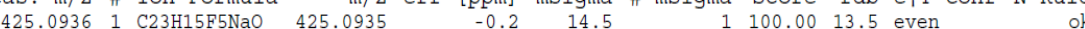




\section{Mass Spectrum SmartFormula Report}

Analysis Info

Analysis Name F:\GAOFENBIAN-2021-1-29\0125_BA7_01_4024.d

Method LC_NO UV_P50-1500_6MIN.m

Sample Name $01 \overline{2} 5$

Acquisition D 2021-01-28 4:14:01

Comment

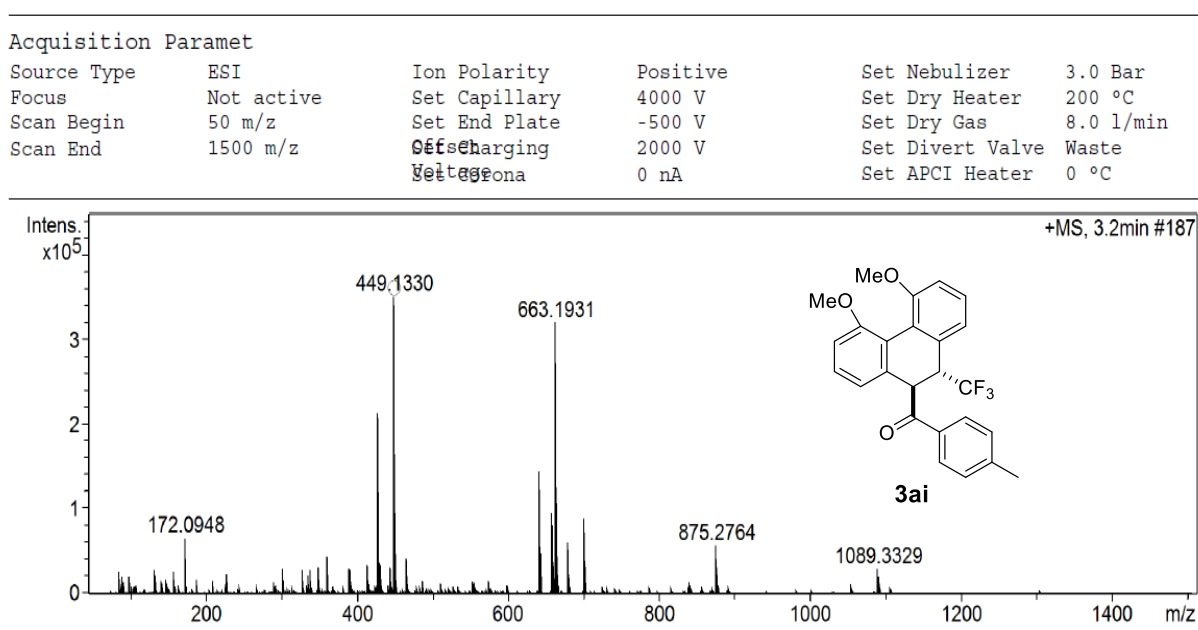

Meas. $\mathrm{m} / \mathrm{z}$ \# Ion Formula

$\mathrm{m} / \mathrm{z}$ err [ppm] mSigma \# mSigma Score $\mathrm{rdb}$ ei¥ Conf $\mathrm{N}$-Rule

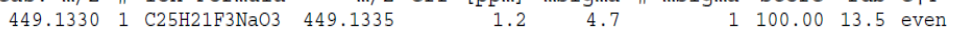

Operator Demo User

Instrumen compact

8255754.2017

Acquisition Paramet

$4000 \mathrm{~V}$

$.0 \mathrm{l} / \mathrm{min}$

ule

\section{Mass Spectrum SmartFormula Report}

Analysis Info

Acquisition D 2020-12-22 16:29:29

Analysis Name F:\GAO FEN BIAN(YANG LING YUN) \GAOFENBIAN-2020-12-24\1221_BE5_01_2686.d

Method LC_NO UV_P50-1500_6MIN.m

Operator Demo User

Sample Name 1221

Instrumen compact

8255754.2017

Comment

Acquisition Paramet

Source Type ESI

Focus

Scan Begin

Not activ

Not activ $50 \mathrm{~m} / \mathrm{z}$

Scan End

$1500 \mathrm{~m} / \mathrm{z}$

Ion Polarity

Set Capillary

Set End Plate

off seharging

Positive

$4000 \mathrm{~V}$

$-500 \mathrm{~V}$

$2000 \mathrm{~V}$

$0 \mathrm{nA}$

Set Nebulizer $\quad 3.0 \mathrm{Bar}$

Set Dry Heater $200^{\circ} \mathrm{C}$

Set Dry Gas $\quad 8.01 / \mathrm{min}$

Set Divert Valve Waste

Set APCI Heater $0{ }^{\circ} \mathrm{C}$

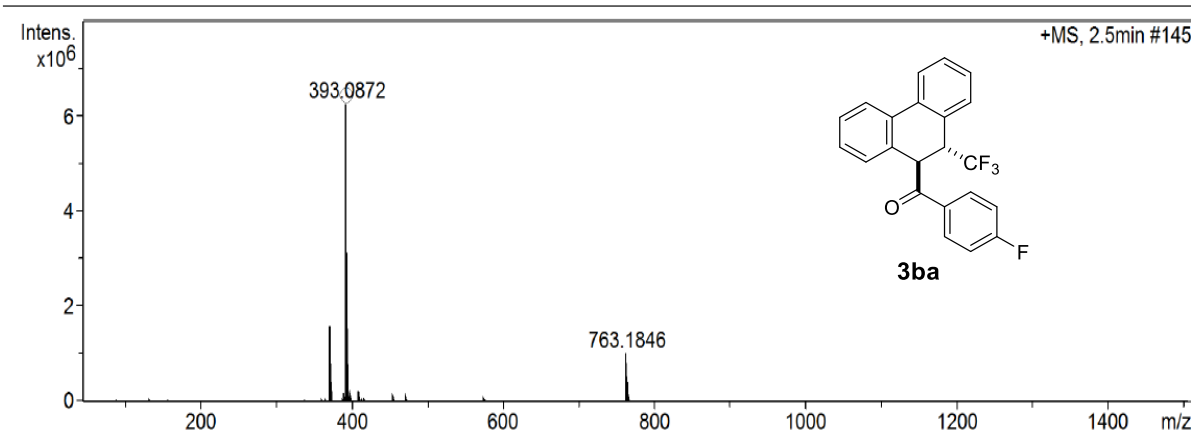

Meas. $\mathrm{m} / \mathrm{z}$ \# Ion Formul

$393.0872 \quad 1$ C22H14F4NaO

$\mathrm{m} / \mathrm{z}$ err [ppm] mSigma \# mSigma Score rdb ei¥ Conf $\mathrm{N}$-Rule

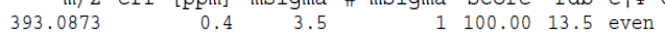




\section{Mass Spectrum SmartFormula Report}

Analysis Info

Acquisition D 2020-12-22 12:24:02

Analysis Name F:\GAO FEN BIAN(YANG LING YUN) \GAOFENBIAN-2020-12-24\1221_BA4_01_2653.d

Method LC_NO UV_P50-1500_6MIN.m Operator Demo User

Sample Name $12 \overline{2} 1-$ Instrumen compact

8255754.2017

Comment

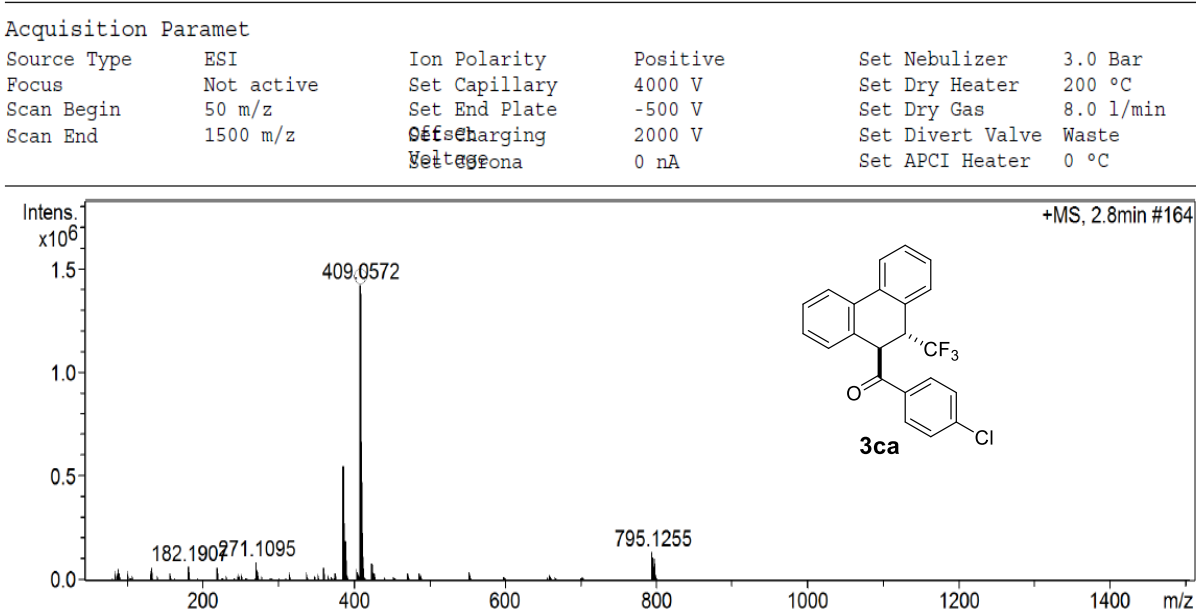

Meas. m/z \# Ion Formula $\mathrm{m} / \mathrm{z}$ err [ppm] mSigma \# mSigma Score rdb ei¥ Conf $\mathrm{N}-\mathrm{Rule}$

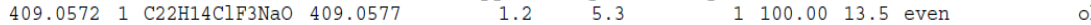

\section{Mass Spectrum SmartFormula Report}

Analysis Info

Acquisition D 2020-12-22 15:51:59

Analysis Name F:\GAO FEN BIAN(YANG LING YUN) \GAOFENBIAN-2020-12-24\1221_BD8_01_2681.d

Method LC_NO UV_P50-1500_6MIN.m Operator Demo User

Sample Name $12 \overline{21} \quad$ Instrumen compact

Comment

Acquisition Paramet

$\begin{array}{llllll}\text { Source Type } & \text { ESI } & \text { Ion Polarity } & \text { Positive } & \text { Set Nebulizer } & 3.0 \mathrm{Bar} \\ \text { Focus } & \text { Not active } & \text { Set Capillary } & 4000 \mathrm{~V} & \text { Set Dry Heater } & 200^{\circ} \mathrm{C} \\ \text { Scan Begin } & 50 \mathrm{~m} / \mathrm{z} & \text { Set End Plate } & -500 \mathrm{~V} & \text { Set Dry Gas } & 8.0 \mathrm{l} / \mathrm{min} \\ \text { Scan End } & 1500 \mathrm{~m} / \mathrm{z} & \text { OEf } & \text { Sethetharging } & 2000 \mathrm{~V} & \text { Set Divert Valve Waste } \\ & & 0 \mathrm{nA} & \text { Set APCI Heater } 0{ }^{\circ} \mathrm{C}\end{array}$

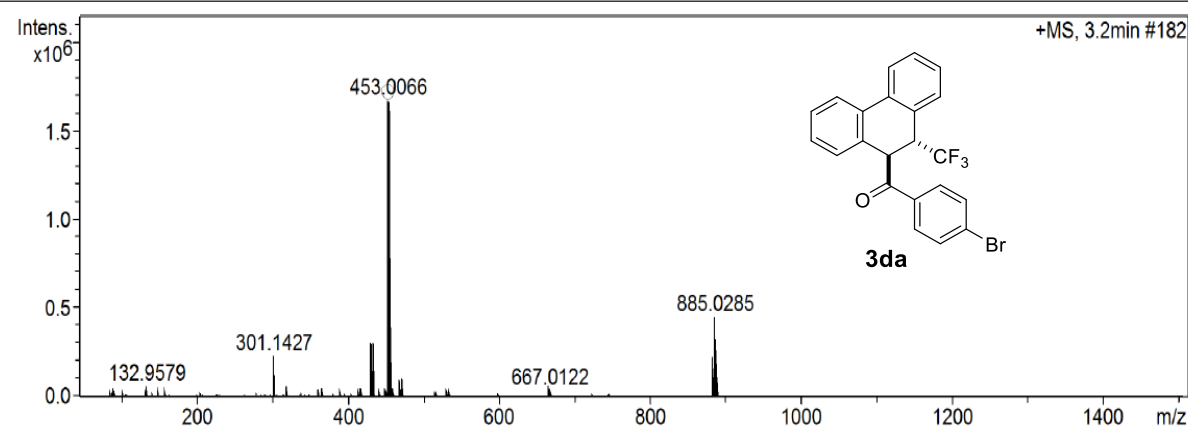

Meas. $\mathrm{m} / \mathrm{z}$ \# Ion Formula $\mathrm{m} / \mathrm{z}$ err [ppm] mSigma \# mSigma Score rdb ei¥ Conf $\mathrm{N}-\mathrm{Rule}$

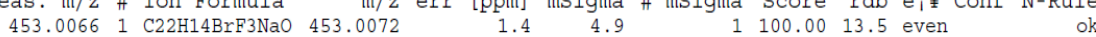




\section{Mass Spectrum SmartFormula Report}

Analysis Info

Acquisition D 2020-12-22 15:37:15

Analysis Name F:\GAO FEN BIAN(YANG LING YUN) \GAOFENBIAN-2020-12-24\1221_BD6_01_2679.d

Method LC_NO UV_P50-1500_6MIN.m Operator Demo User

Sample Name 122

Instrumen compact

8255754.2017

Comment

\begin{tabular}{llllll}
\hline Acquisition & Paramet & & & \\
Source Type & ESI & Ion Polarity & Positive & Set Nebulizer & 3.0 Bar \\
Focus & Not active & Set Capillary & $4000 \mathrm{~V}$ & Set Dry Heater & $200^{\circ} \mathrm{C}$ \\
Scan Begin & $50 \mathrm{~m} / \mathrm{z}$ & Set End Plate & $-500 \mathrm{~V}$ & Set Dry Gas & $8.0 \mathrm{l} / \mathrm{min}$ \\
Scan End & $1500 \mathrm{~m} / \mathrm{z}$ & Offetharging & $2000 \mathrm{~V}$ & Set Divert Valve Waste \\
& & Settegrona & $0 \mathrm{nA}$ & Set APCI Heater $00^{\circ} \mathrm{C}$ \\
\hline
\end{tabular}

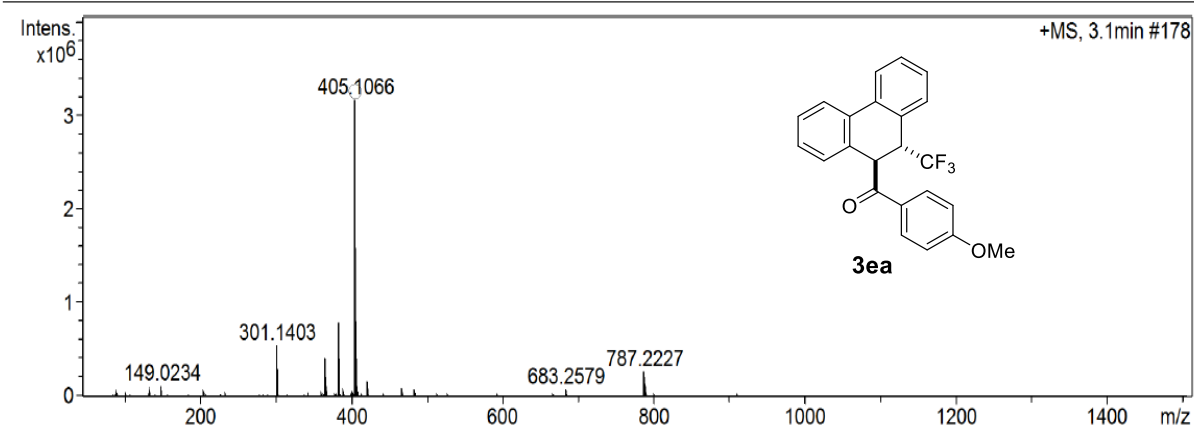

Meas. $\mathrm{m} / \mathrm{z}$ \# Ion Formula $\mathrm{m} / \mathrm{z}$ err [ppm] mSigma \# mSigma Score rdb ei¥ Conf $\mathrm{N}$-Rule

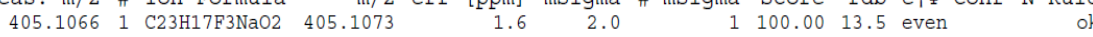

\section{Mass Spectrum SmartFormula Report}

Analysis Info

Acquisition D 2020-12-22 16:07:11 Analysis Name F: \GAO FEN BIAN(YANG LING YUN) \GAOFENBIAN-2020-12-24\1221_BE2_01_2683.d Method LC_NO UV_P50-1500_6MIN.m Operator Demo User

Sample Name $12 \overline{2} 1 \quad$ Instrumen compact

8255754.2017

Comment

Acquisition Paramet

Source Type ESI

Fource Type ESI

Scan Begin $\quad 50 \mathrm{~m} / \mathrm{z}$

Scan End $\quad 1500 \mathrm{~m} / \mathrm{z}$

Ion Polarity

Set Capillary

Set End Plate

offselharging

$4000 \mathrm{~V}$
$-500 \mathrm{~V}$

Seztagena

Set Nebulizer $\quad 3.0 \mathrm{Bar}$

Set Dry Heater $200{ }^{\circ} \mathrm{C}$

Set Dry Gas $\quad 8.01 / \mathrm{min}$

Divert Valve Waste

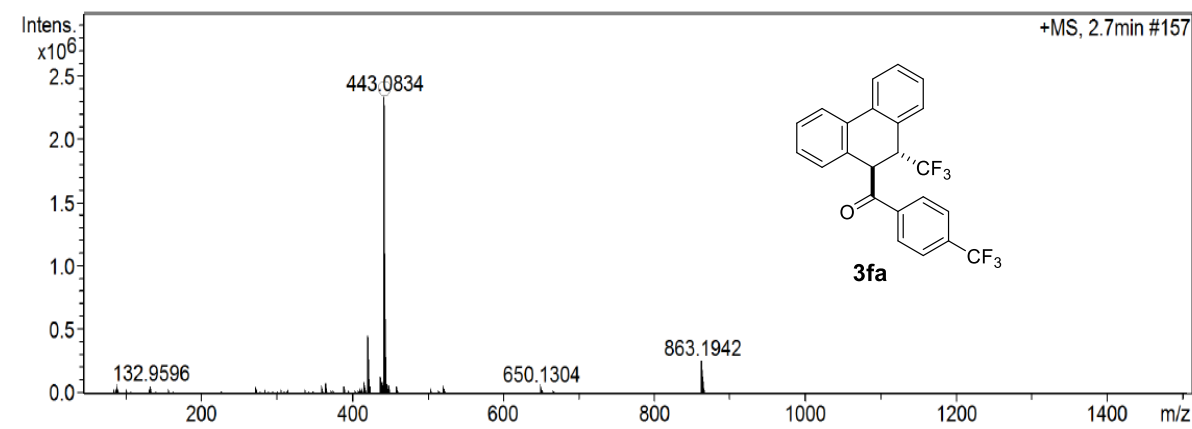

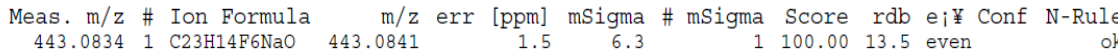
$443.08341 \mathrm{C} 23 \mathrm{H} 14 \mathrm{~F} 6 \mathrm{NaO}$

443.084

1100.0013 .5 even 


\section{Mass Spectrum SmartFormula Report}

Analysis Info

Acquisition D 2020-12-22 14:37:24

Analysis Name F: \GAO FEN BIAN(YANG LING YUN) \GAOFENBIAN-2020-12-24\1221_BC6 01_2671.d

Method LC_NO UV_P50-1500_6MIN.m

Operator Demo User

Sample Name 1221

Instrumen compact

8255754.2017

Comment

Acquisition Paramet

Source Type

Focus

Scan Begin $\quad 50 \mathrm{~m} / \mathrm{z}$

Scan End $\quad 1500 \mathrm{~m} / \mathrm{z}$

Ion Polarity

Set Capillary

Set End Plate

off selbarging

Positive

$4000 \mathrm{~V}$

$-500 \mathrm{~V}$

$2000 \mathrm{~V}$

Set Dry Heater $200^{\circ} \mathrm{C}$

Set Dry Gas $\quad 8.01 / \mathrm{min}$

Set Divert Valve Waste

\begin{tabular}{rr}
\hline Intens. \\
$\times 10^{6}-$
\end{tabular}

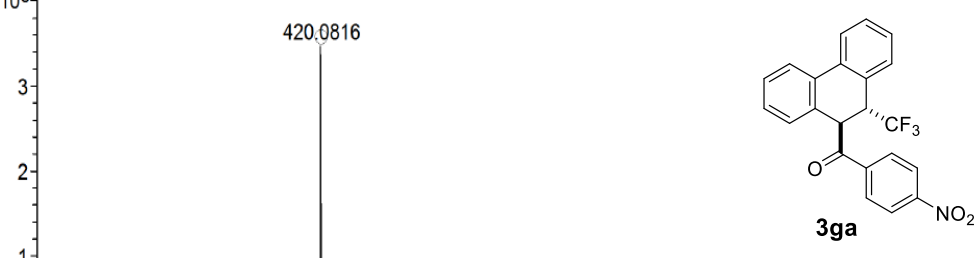

132.9618

615.6205

817.1740

200

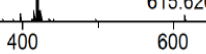

800

1000

1200

$1400 \mathrm{~m} / \mathrm{z}$

Meas. $\mathrm{m} / \mathrm{z}$ \# Ion Formul

$420.0816 \quad 1$ C22H14F3NNaO3 420.08

$\mathrm{m} / \mathrm{z}$ err [ppm] mSigma \# mSigma Score $r d b$ ej¥ Conf $\mathrm{N}$-Rule

\section{Mass Spectrum SmartFormula Report}

Analysis Info

Acquisition D 2020-12-22 13:37:32

Analysis Name F: \GAO FEN BIAN(YANG LING YUN) \GAOFENBIAN-2020-12-24\1221_BB6_01_2663.d

Method LC_NO UV_P50-1500_6MIN.m Operator Demo User

Sample Name $122 \overline{2} \quad$ Instrumen compact

Comment

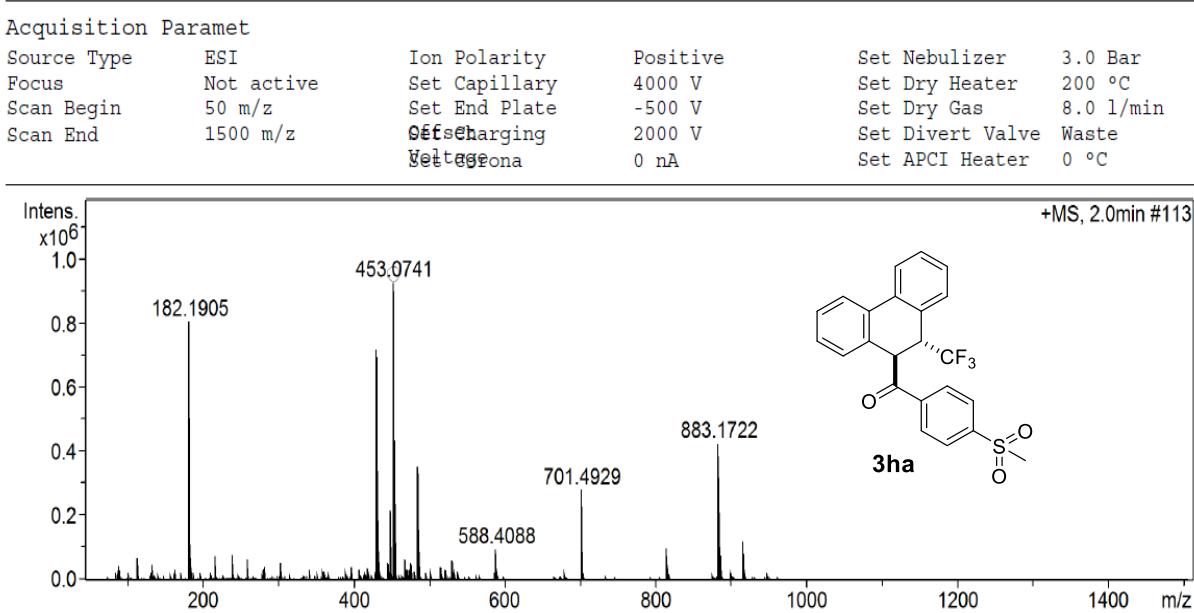

Meas. $\mathrm{m} / \mathrm{z}$ \# Ion Formula $\mathrm{m} / \mathrm{z}$ err [ppm] mSigma \# mSigma Score $\mathrm{rdb}$ e $¥$ ¥ Conf $\mathrm{N}-\mathrm{Rule}$ $\begin{array}{lllllllll}453.0741 & 1 & \mathrm{C} 23 \mathrm{H} 17 \mathrm{~F} 3 \mathrm{NaO} S \mathrm{~S} & 453.0743 & 0.4 & 1.5 & 1 & 100.00 & 13.5 \text { even }\end{array}$ 


\section{Mass Spectrum SmartFormula Report}

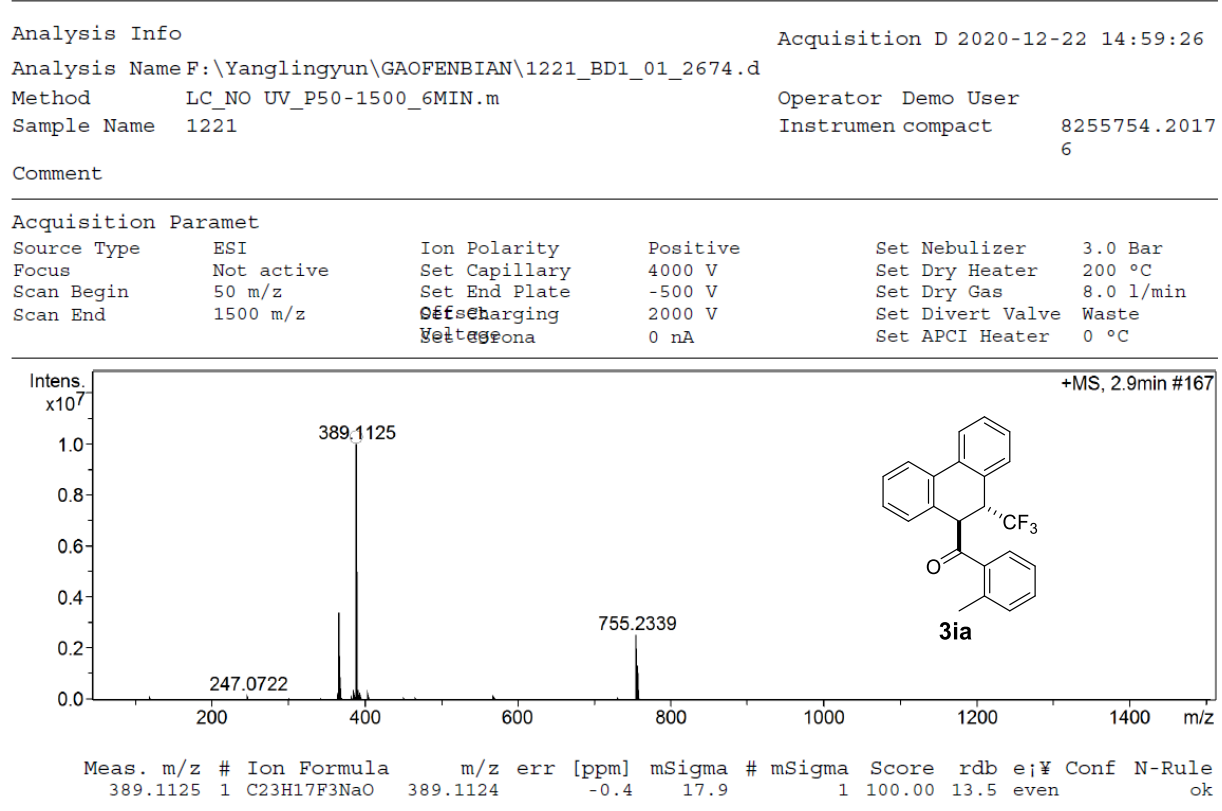

\section{Mass Spectrum SmartFormula Report}

Analysis Info

Acquisition D 2020-12-22 15:21:46

Analysis Name F: \GAO FEN BIAN(YANG LING YUN) \GAOFENBIAN-2020-12-24\1221_BD4_01_2677.d

Method LC_NO UV_P50-1500_6MIN.m Operator Demo User

Sample Name $12 \overline{2} 1 \quad$ Instrumen compact

Comment

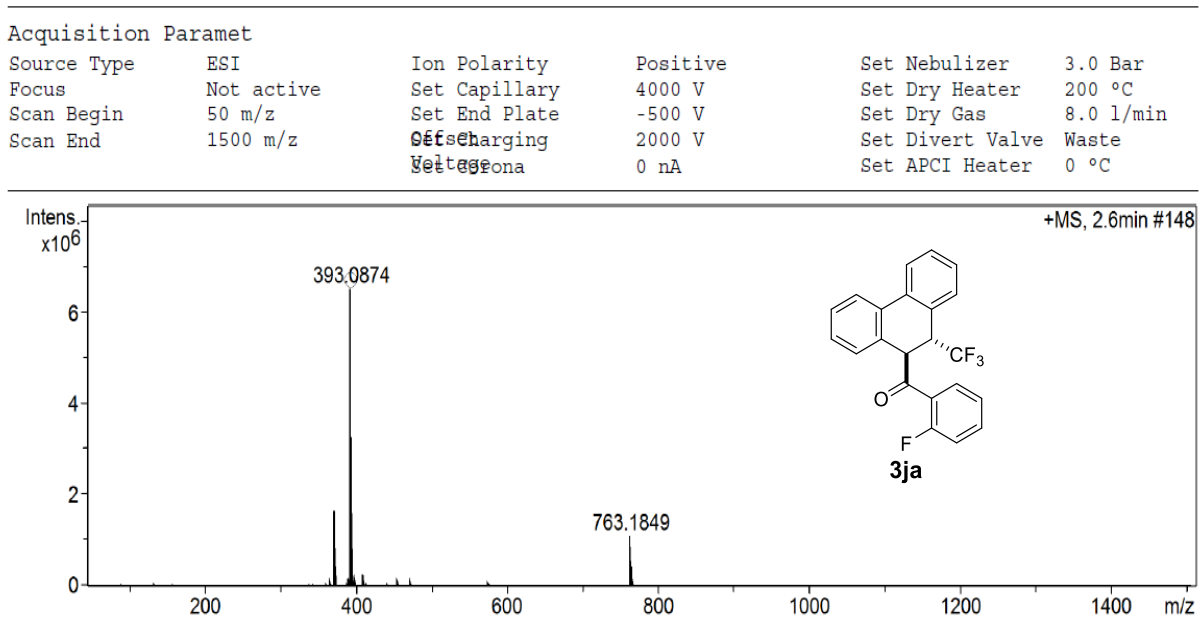

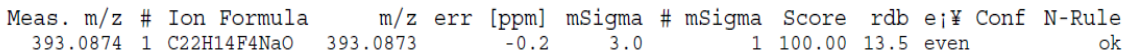
393.0873

1100.0013 .5 even 


\section{Mass Spectrum SmartFormula Report}

Analysis Info Acquisition D 2020-12-22 14:14:51

Analysis Name F: \GAO FEN BIAN(YANG LING YUN) \GAOFENBIAN-2020-12-24\1221_BC3_01_2668.d

Method LC_NO UV_P50-1500_6MIN.m Operator Demo User

Sample Name $1221 \quad$ Instrumen compact

8255754.2017

Comment

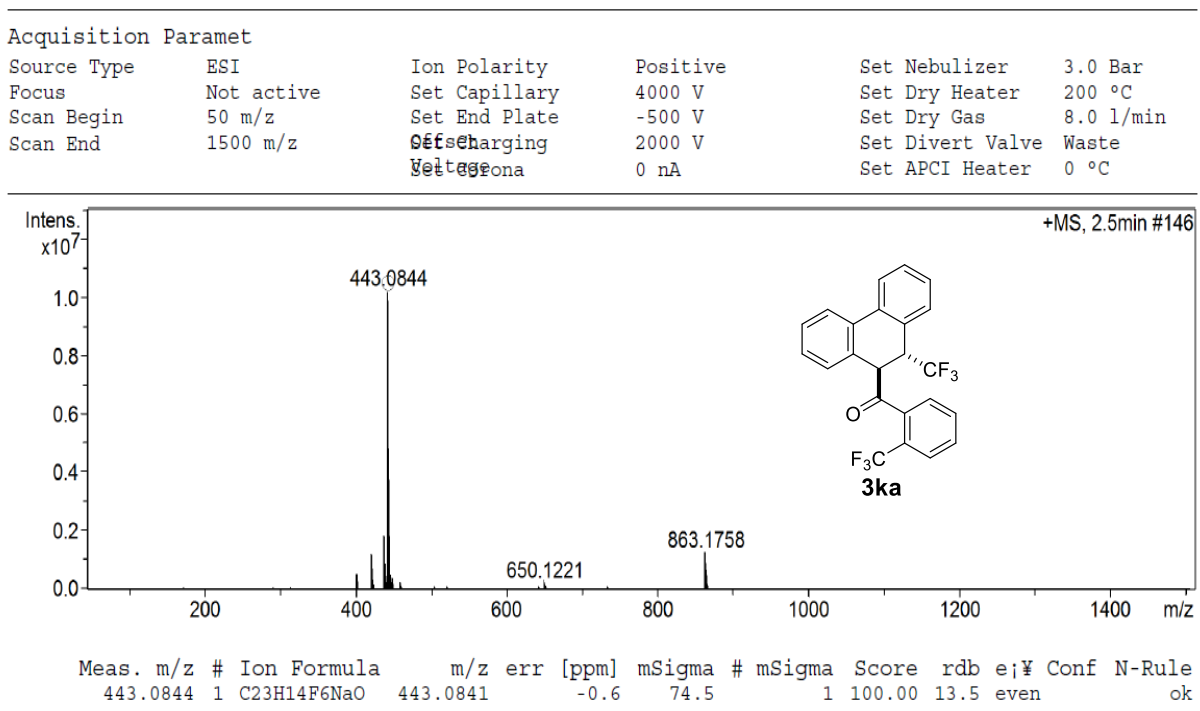

\section{Mass Spectrum SmartFormula Report}

Analysis Info

Acquisition D 2020-12-22 13:00:47

Analysis Name F:\GAO FEN BIAN(YANG LING YUN) \GAOFENBIAN-2020-12-24\1221_BB1_01_2658.d

Method LC_NO UV_P50-1500_6MIN.m Operator Demo User

Sample Name $1221 \quad$ Instrumen compact

8255754.2017

Comment

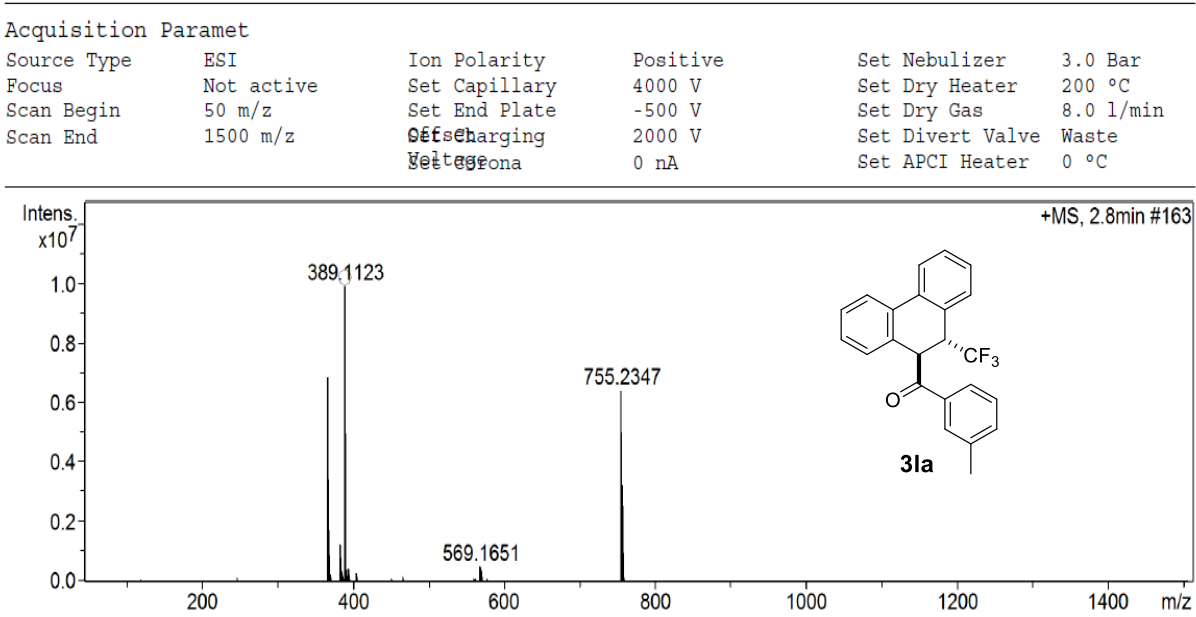

Meas. $\mathrm{m} / \mathrm{z}$ \# Ion Formula $\mathrm{m} / \mathrm{z}$ err [ppm] mSigma \# mSigma Score rdb $e_{i} ¥$ Conf $\mathrm{N}-\mathrm{Rule}$ $389.11231 \mathrm{C} 23 \mathrm{H} 17 \mathrm{~F} 3 \mathrm{NaO} \quad 389.1124-0.3 \quad 16.9-13000$ 


\section{Mass Spectrum SmartFormula Report}

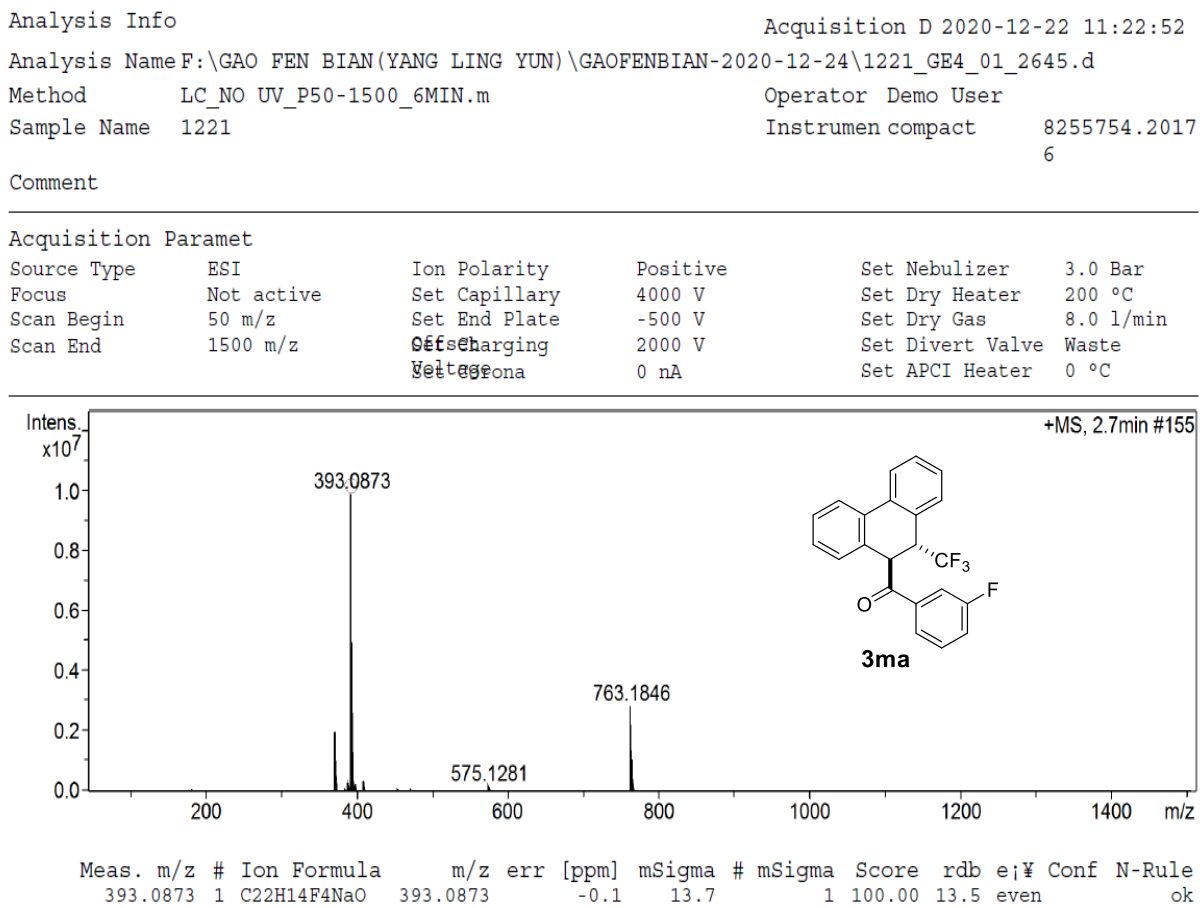

\section{Mass Spectrum SmartFormula Report}

Analysis Info

Acquisition D 2020-12-22 13:08:09

Analysis Name F:\GAO FEN BIAN(YANG LING YUN) \GAOFENBIAN-2020-12-24\1221_BB2_01_2659.d

Method LC_NO UV_P50-1500_6MIN.m Operator Demo User

Sample Name $1221 \quad$ Instrumen compact

8255754.2017

Comment

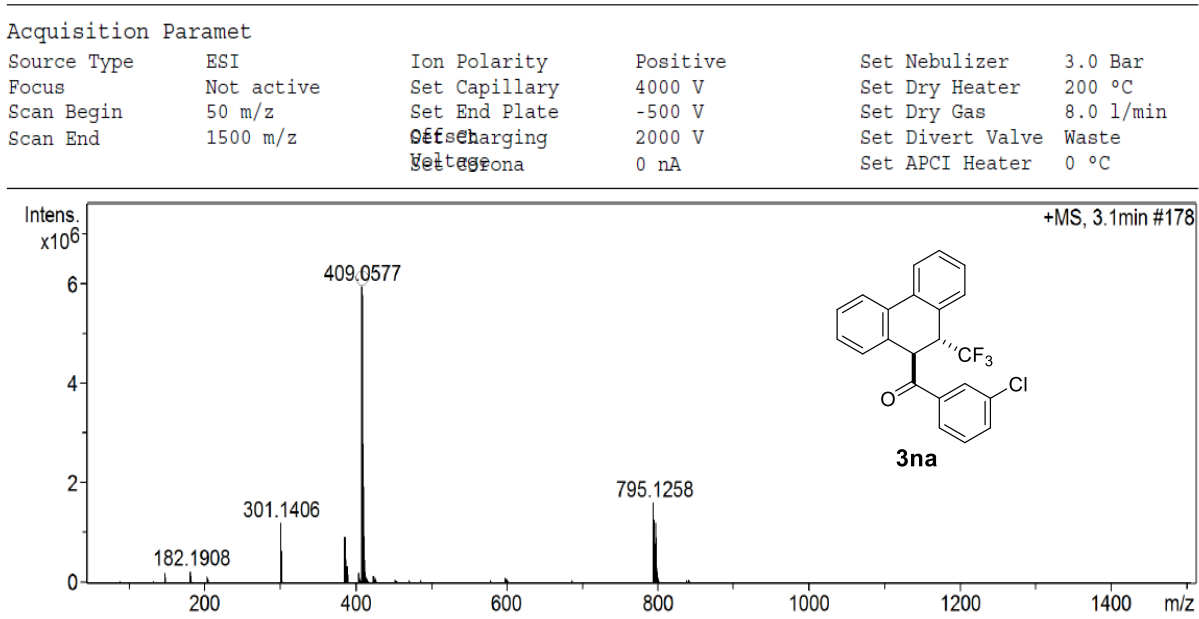

Meas. $\mathrm{m} / \mathrm{z}$ \# Ion Formula $\mathrm{m} / \mathrm{z}$ err [ppm] mSigma \# mSigma Score rdb ei¥ Conf $\mathrm{N}-\mathrm{Rule}$ $\begin{array}{lllllllll}409.0577 & 1 & \mathrm{C} 22 \mathrm{H} 14 \mathrm{ClF} 3 \mathrm{NaO} & 409.0577 & 0.2 & 5.9 & 1 & 100.00 & 13.5 \text { even }\end{array}$ 


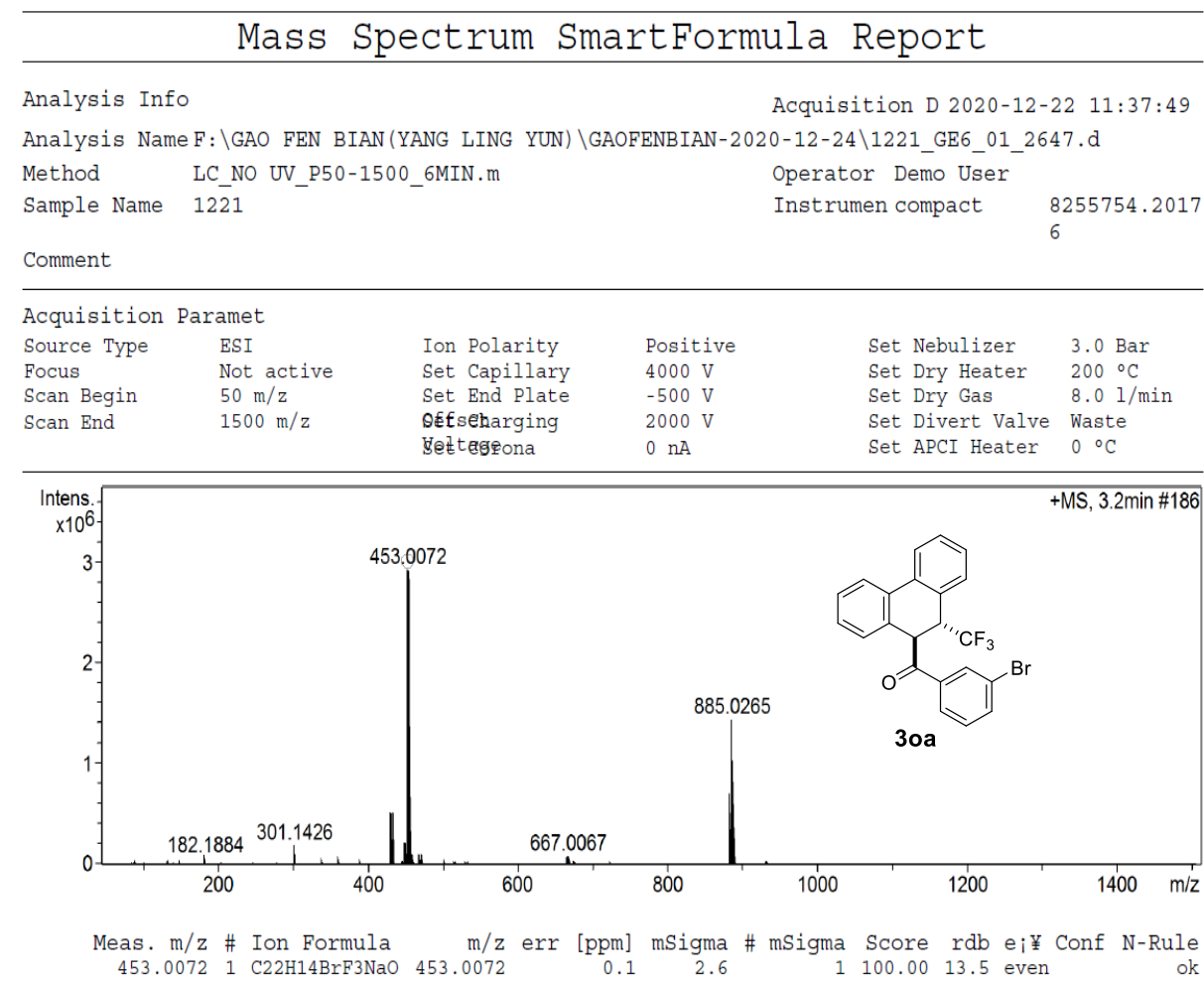

\section{Mass Spectrum SmartFormula Report}

Analysis Info

Acquisition D 2020-12-22 16:14:32

Analysis Name F:\GAO FEN BIAN(YANG LING YUN) \GAOFENBIAN-2020-12-24\1221_BE3_01_2684.d

Method LC_NO UV_P50-1500_6MIN.m Operator Demo User

Sample Name $12 \overline{2} 1$ Instrumen compact

8255754.2017

Comment

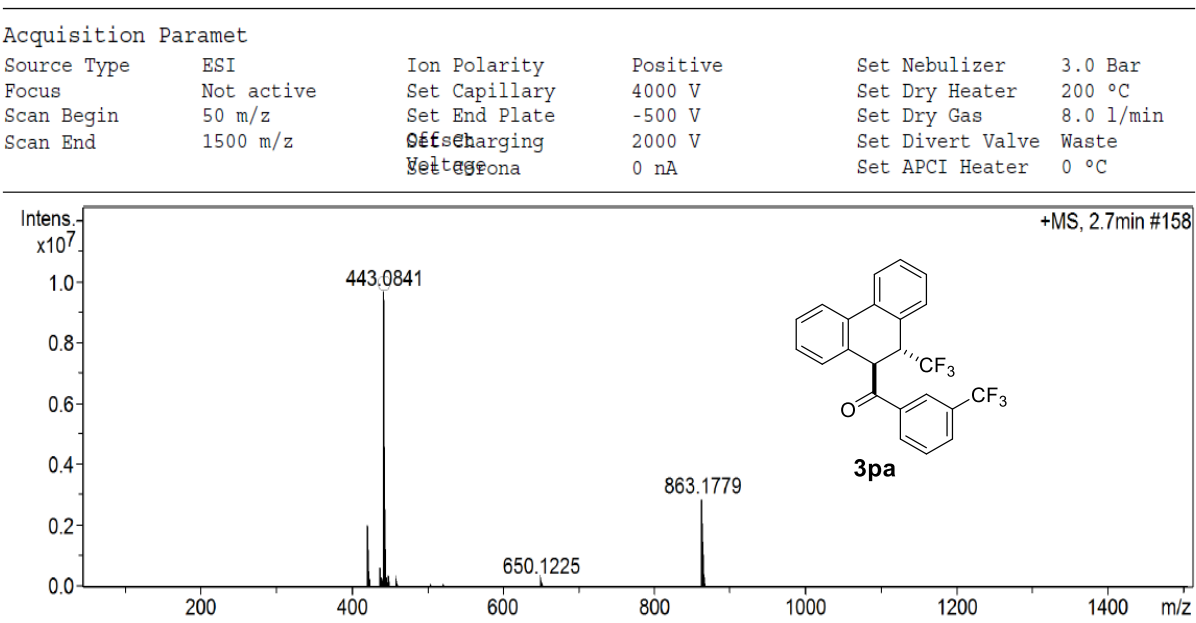

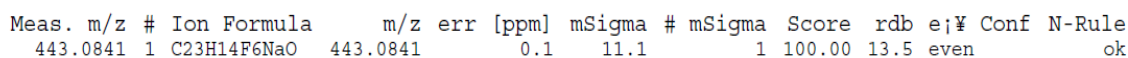

1100.0013 .5 even 


\section{Mass Spectrum SmartFormula Report}

Analysis Info

Acquisition D 2020-12-22 13:15:29

Analysis Name F:\GAO FEN BIAN(YANG LING YUN) \GAOFENBIAN-2020-12-24\1221_BB3 01 2660.d

Method LC_NO UV_P50-1500_6MIN.m Operator Demo User

Sample Name 1221 Instrumen compact

8255754.2017

Comment

\begin{tabular}{llllll}
\hline Acquisition & Paramet & & & & \\
Source Type & ESI & Ion Polarity & Positive & Set Nebulizer & $3.0 \mathrm{Bar}$ \\
Focus & Not active & Set Capillary & $4000 \mathrm{~V}$ & Set Dry Heater & $200^{\circ} \mathrm{C}$ \\
Scan Begin & $50 \mathrm{~m} / \mathrm{z}$ & Set End Plate & $-500 \mathrm{~V}$ & Set Dry Gas & $8.01 / \mathrm{min}$ \\
Scan End & $1500 \mathrm{~m} / \mathrm{z}$ & OEf & Wetharging & $2000 \mathrm{~V}$ & Set Divert Valve Waste \\
& & & Settagena & $0 \mathrm{nA}$ & Set APCI Heater $0{ }^{\circ} \mathrm{C}$
\end{tabular}

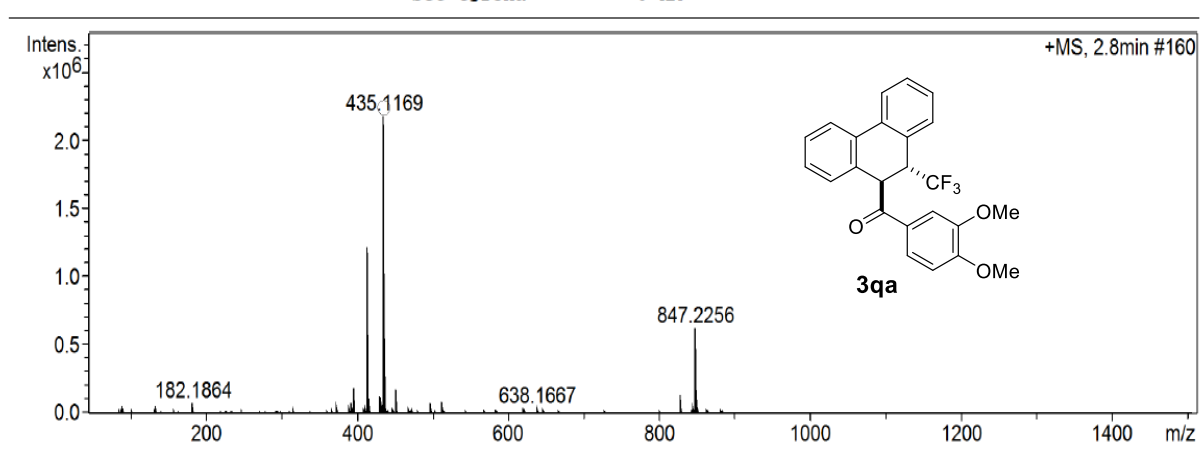

Meas. m/z \# Ion Formula $\mathrm{m} / \mathrm{z}$ err [ppm] mSigma \# mSigma Score rdb e $¥ ¥$ Conf $\mathrm{N}-\mathrm{Rule}$

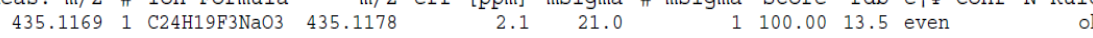

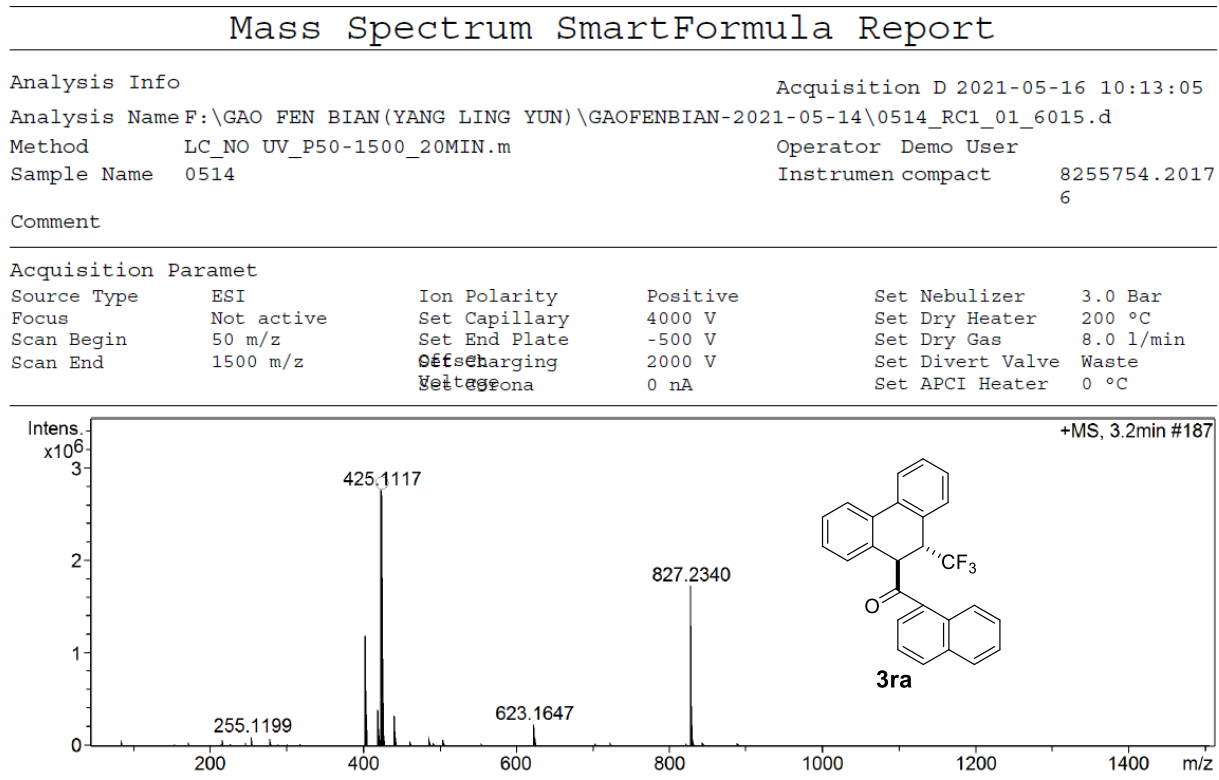

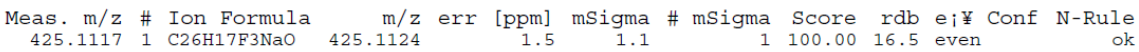




\section{Mass Spectrum SmartFormula Report}

Analysis Info

Acquisition D 2020-12-22 14:44:45

Analysis Name F:\GAO FEN BIAN(YANG LING YUN) \GAOFENBIAN-2020-12-24\1221_BC7_01_2672.d

Method LC_NO UV_P50-1500_6MIN.m

Operator Demo User

Instrumen compact $\quad 8255754.2017$

Sample Name $1221 \quad$ Instrumen compact

Comment

Acquisition Paramet

$\begin{array}{llllll}\text { Source Type } & \text { ESI } & \text { Ion Polarity } & \text { Positive } & \text { Set Nebulizer } & 3.0 \mathrm{Bar} \\ \text { Focus } & \text { Not active } & \text { Set Capillary } & 4000 \mathrm{~V} & \text { Set Dry Heater } & 2000^{\circ} \mathrm{C} \\ \text { Scan Begin } & 50 \mathrm{~m} / \mathrm{z} & \text { Set End Plate } & -500 \mathrm{~V} & \text { Set Dry Gas } & 8.0 \mathrm{l} / \mathrm{min} \\ \text { Scan End } & 1500 \mathrm{~m} / \mathrm{z} & \text { Oefsetharging } & 2000 \mathrm{~V} & \text { Set Divert Valve Waste } \\ & & \text { Settergena } & 0 \mathrm{nA} & \text { Set APCI Heater } 0{ }^{\circ} \mathrm{C}\end{array}$

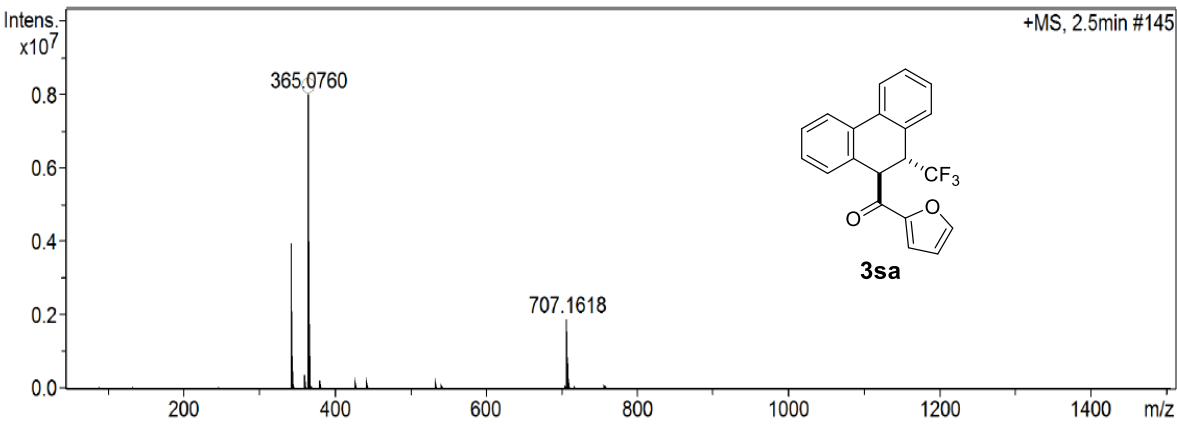

Meas. $\mathrm{m} / \mathrm{z}$ \# Ion Formula $\mathrm{m} / \mathrm{z}$ err [ppm] mSigma \# mSigma Score rdb ei¥ Conf $\mathrm{N}$-Rule

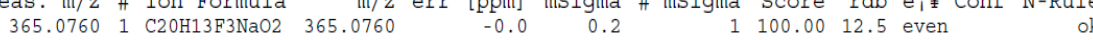

\section{Mass Spectrum SmartFormula Report}

Analysis Info

Acquisition D 2021-05-16 11:17:23

Analysis Name F:\GAO FEN BIAN(YANG LING YUN) \GAOFENBIAN-2021-05-14\0514_RC4_01 6018.d

Method LC_NO UV_P50-1500_20MIN.m Operator Demo User

Sample Name $0514 \quad$ Instrumen compact

8255754.2017

Comment

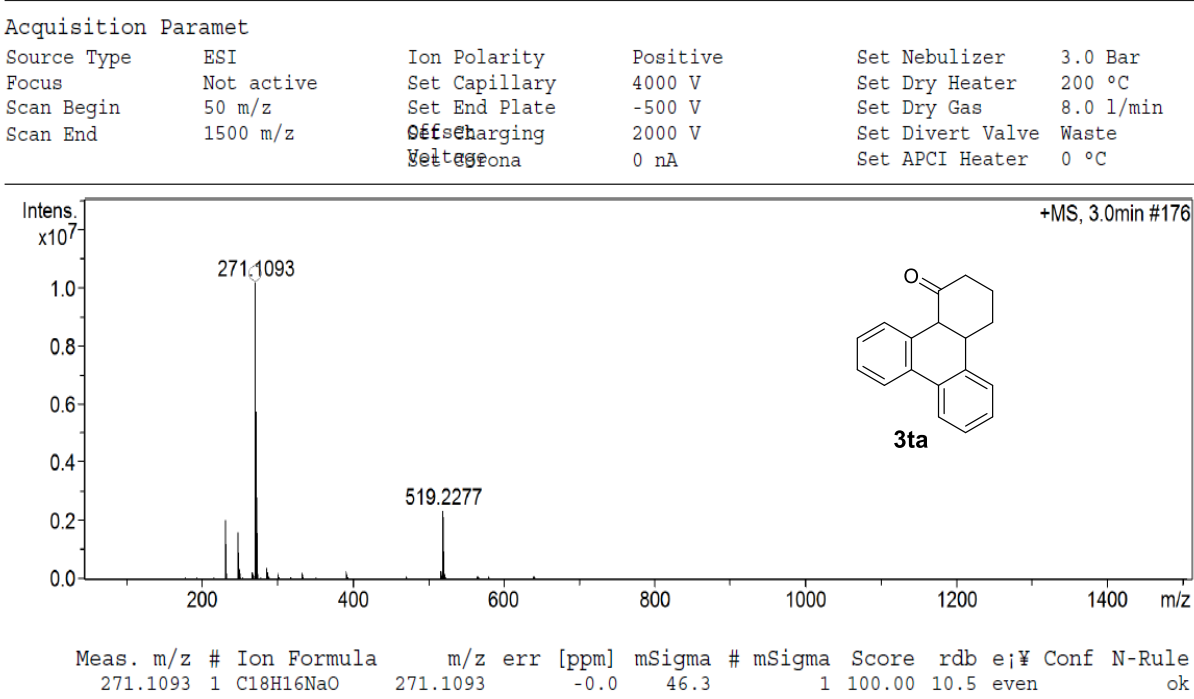




\section{Mass Spectrum SmartFormula Report}

Analysis Info

Acquisition D 2020-12-22 11:52:48

Analysis Name F: \GAOFENBIAN-2020-12-24\1221_GE8_01_2649.d

Method LC_NO UV_P50-1500_6MIN.m Operator Demo User

Sample Name $1221 \quad$ Instrumen compact Instrumen compact

8255754.2017

Comment

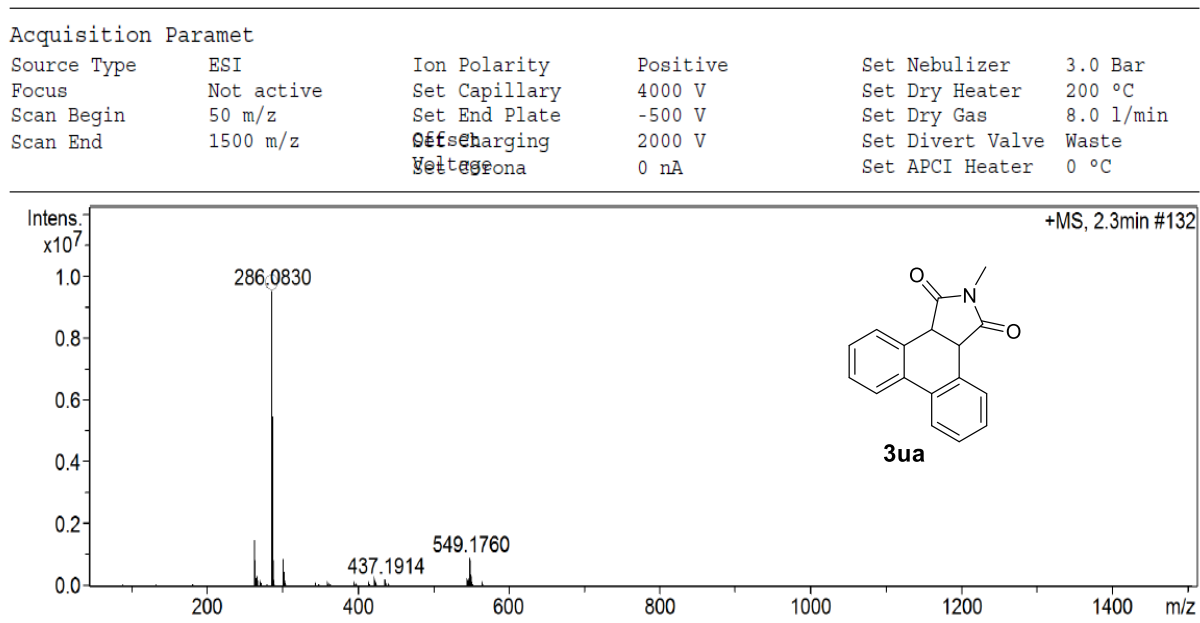

Meas. $\mathrm{m} / \mathrm{z}$ \# Ion Formula $\mathrm{m} / \mathrm{z}$ err [ppm] mSigma \# mSigma Score rdb ei¥ Conf N-Rule

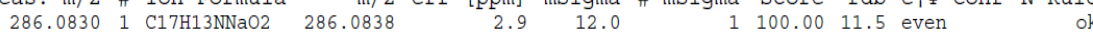

\section{Mass Spectrum SmartFormula Report}

Analysis Info

Acquisition D 2020-12-22 11:08:12

Analysis Name F: \GAOFENBIAN-2020-12-24\1221_GE2_01_2643.d

Method LC NO UV P50-1500 6MIN.m

Operator Demo User

Sample Name $1221 \quad$ Instrumen compact

Comment

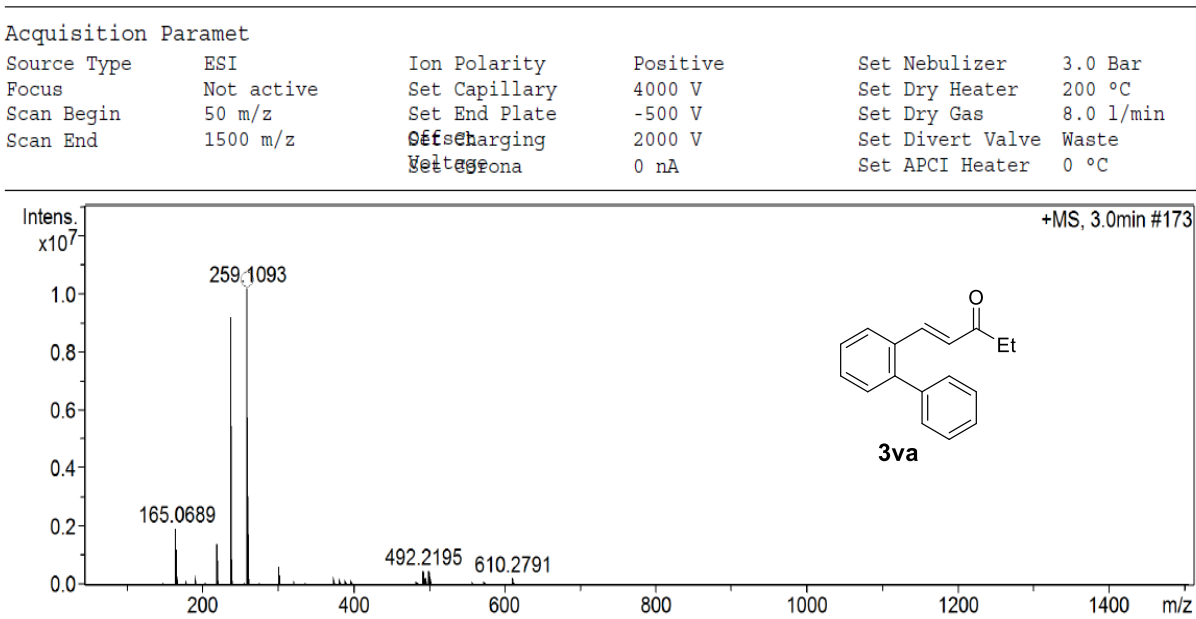

Meas. $\mathrm{m} / \mathrm{z}$ \# Ion Formula $\mathrm{m} / \mathrm{z}$ err [ppm] mSigma \# mSigma Score rdb ei¥ Conf $\mathrm{N}$-Rule

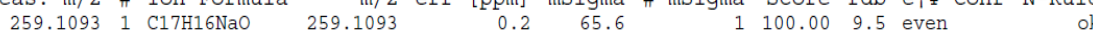




\section{Mass Spectrum SmartFormula Report}

Analysis Info

Acquisition D 2020-12-22 14:30:03

Analysis Name F:\GAO FEN BIAN(YANG LING YUN) \GAOFENBIAN-2020-12-24\1221_BC5_01_2670.d

Method LC_NO UV_P50-1500_6MIN.m Operator Demo User

Sample Name 1221 Instrumen compact

8255754.2017

Comment

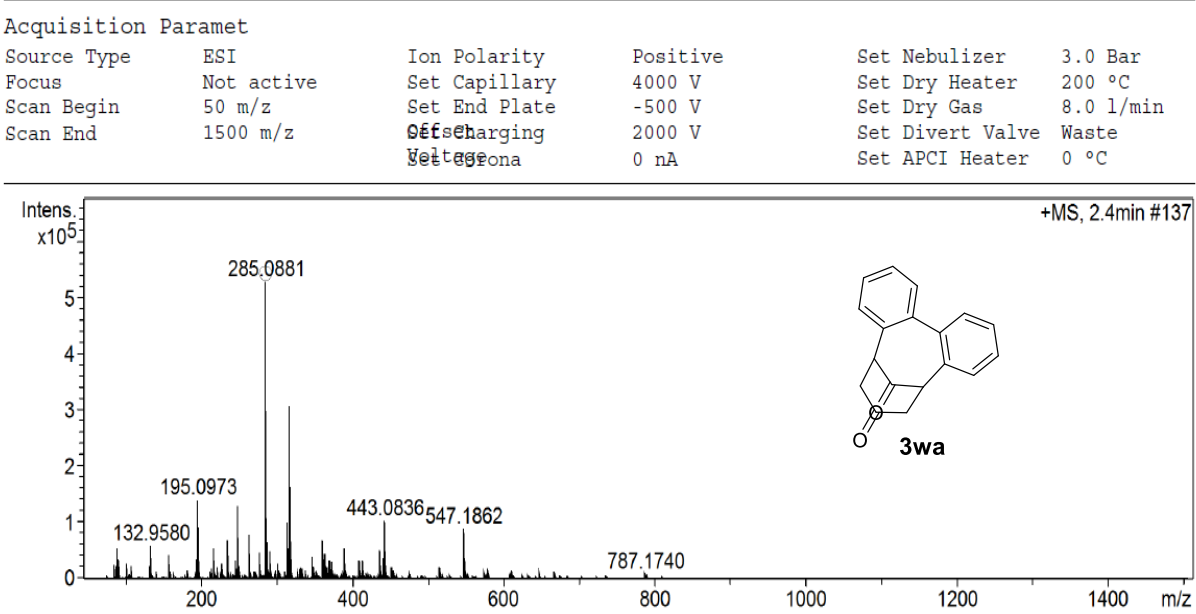

Meas. $\mathrm{m} / \mathrm{z}$ \# Ion Formula $\mathrm{m} / \mathrm{z}$ err [ppm] mSigma \# mSigma Score rdb ei¥ Conf $\mathrm{N}-\mathrm{Rule}$ $285.08811 \mathrm{C} 18 \mathrm{H} 14 \mathrm{NaO} 2$ 285.0886

$1.9 \quad$ r.6

1100.0011 .5 even

\section{Mass Spectrum SmartFormula Report}

Analysis Info

Acquisition D 2020-12-22 13:30:13

Analysis Name F:\GAO FEN BIAN(YANG LING YUN) \GAOFENBIAN-2020-12-24\1221_BB5_01_2662.d
Method
LC NO UV P50-1500 6MIN.m
Operator Demo User

Sample Name 1221

Instrumen compact

8255754.2017

Comment

$\begin{array}{llllll}\text { Acquisition } & \text { Paramet } & & & \\ \text { Source Type } & \text { ESI } & \text { Ion Polarity } & \text { Positive } & \text { Set Nebulizer } & 3.0 \mathrm{Bar} \\ \text { Focus } & \text { Not active } & \text { Set Capillary } & 4000 \mathrm{~V} & \text { Set Dry Heater } & 200^{\circ} \mathrm{C} \\ \text { Scan Begin } & 50 \mathrm{~m} / \mathrm{z} & \text { Set End Plate } & -500 \mathrm{~V} & \text { Set Dry Gas } & 8.0 \mathrm{l} / \mathrm{min} \\ \text { Scan End } & 1500 \mathrm{~m} / \mathrm{z} & \text { Oefseharging } & 2000 \mathrm{~V} & \text { Set Divert Valve Waste } \\ & & \text { Jettagena } & 0 \mathrm{nA} & \text { Set APCI Heater } 0{ }^{\circ} \mathrm{C}\end{array}$

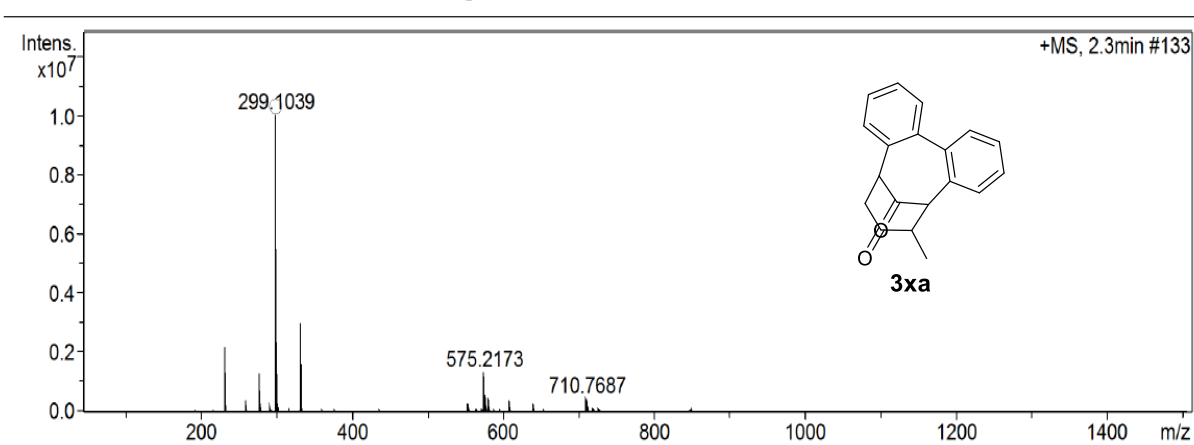

Meas. $\mathrm{m} / \mathrm{z}$ \# Ion Formula $\mathrm{m} / \mathrm{z}$ err [ppm] mSigma \# mSigma Score rdb e $¥ ¥$ Conf $\mathrm{N}-$ Rule

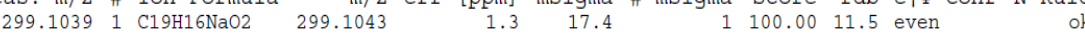




\section{Mass Spectrum SmartFormula Report}

Analysis Info

Acquisition D 2020-12-22 15:21:46

Analysis Name F:\GAO FEN BIAN(YANG LING YUN) \GAOFENBIAN-2020-12-24\1221_BD4_01_2677.d

Method LC NO UV P50-1500 6MIN.m

Operator Demo User

Sample Name $12 \overline{2} 1$

Instrumen compact

Comment

Acquisition Paramet

Source Type ESI

Scan Begin $\quad 50 \mathrm{~m} / \mathrm{z}$

Set Capillary

Set End Plate $-500 \mathrm{~V}$

$-500 \mathrm{~V}$

Efsebarging

$0 \mathrm{nA}$

Sett taøena

Set Nebulizer $\quad 3.0$ Bar

Set Dry Heater $200^{\circ} \mathrm{C}$

Set Dry Gas $\quad 8.0 \mathrm{l} / \mathrm{min}$

lve Waste

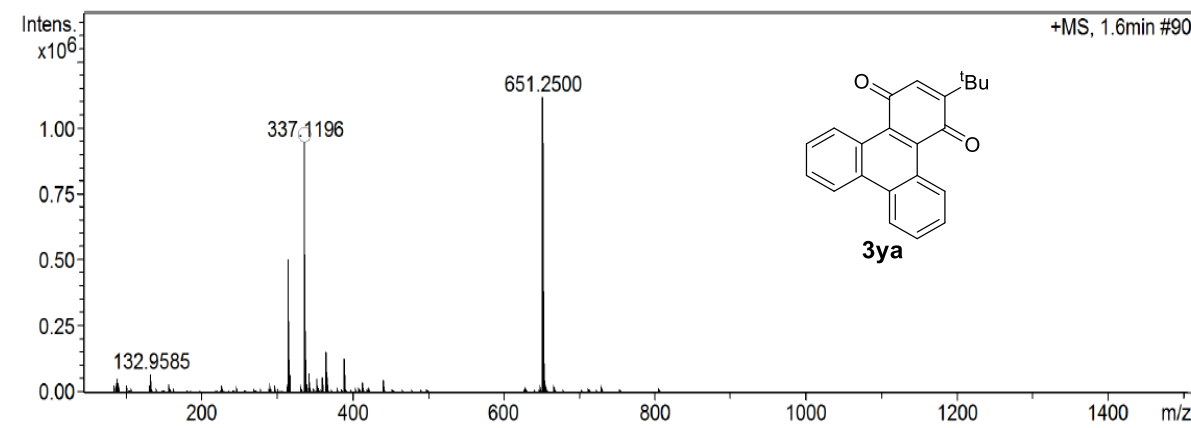

Meas. $\mathrm{m} / \mathrm{z}$ \# Ion Formula $\mathrm{m} / \mathrm{z}$ err [ppm] mSigma \# mSigma Score $r \mathrm{db}$ e $¥$ $¥$ Conf $\mathrm{N}-\mathrm{Rul}$

\section{Mass Spectrum SmartFormula Report}

Analysis Info

Acquisition D 2020-12-22 14:52:06

Analysis Name F: \Yanglingyun \GAOFENBIAN \1221_BC8_01_2673.d

Method

LC_NO UV_P50-1500_6MIN.m

Operator Demo User

Sample Name 1221

Instrumen compact

8255754.2017

Comment

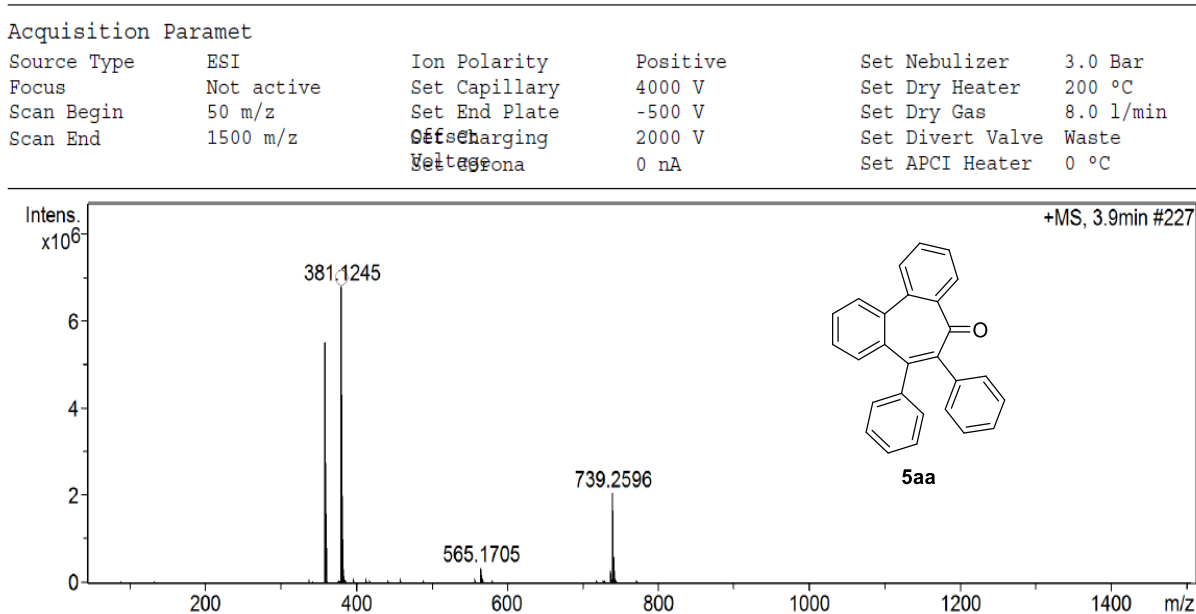

Meas. $\mathrm{m} / \mathrm{z}$ \# Ion Formula

$\mathrm{m} / \mathrm{z}$ err [ppm] mSigma \# mSigma Score rdb ei¥ Conf N-Rule $381.12451 \mathrm{C} 27 \mathrm{H} 18 \mathrm{NaO}$ 381.1250

$\begin{array}{ll}1.3 & 0.5\end{array}$

1100.0018 .5 even 


\section{Mass Spectrum SmartFormula Report}

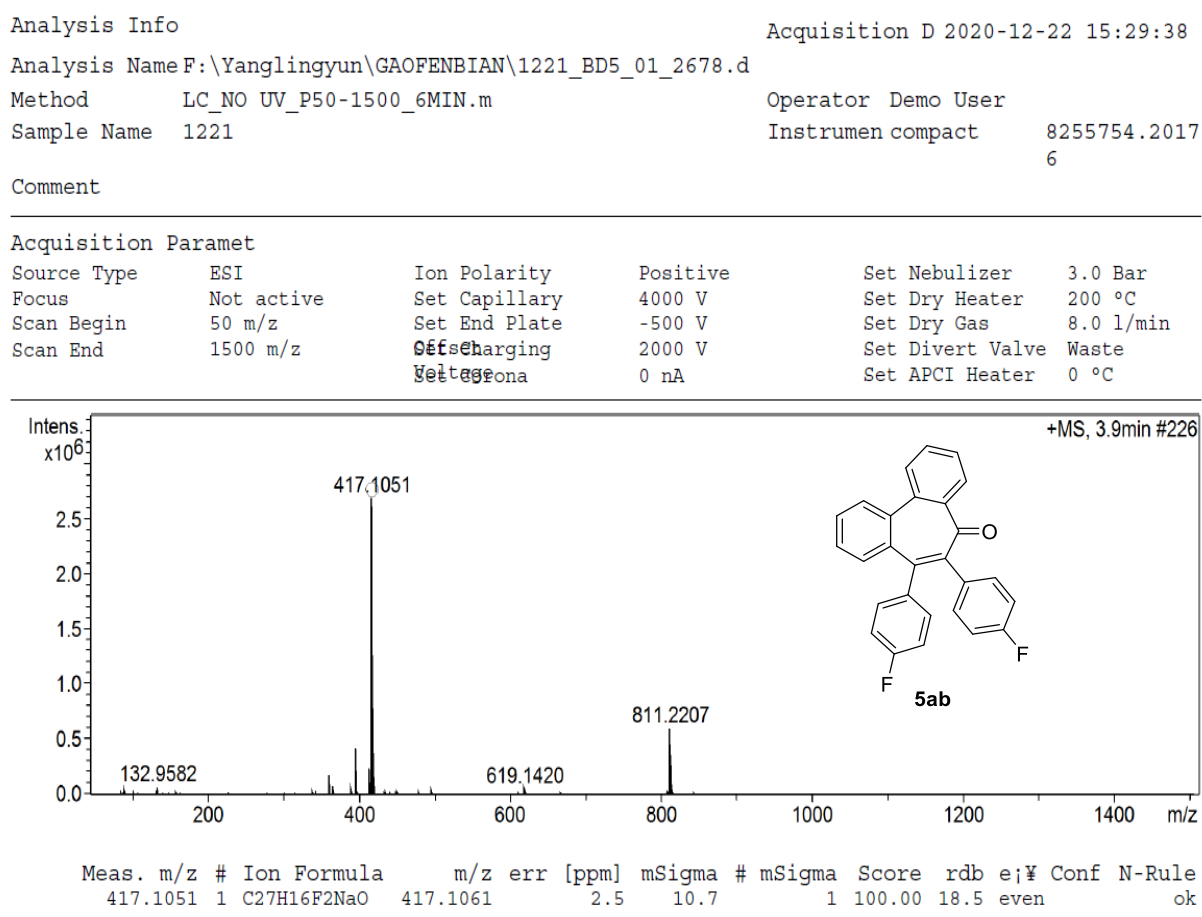

\section{Mass Spectrum SmartFormula Report}

Analysis Info

Acquisition D 2021-06-18 20:53:07

Analysis Name F:\GAO FEN BIAN(YANG LING YUN) \GAOFENBIAN-2021-06-16\0616_RD2_01_6571.d

Method LC_NO UV_P50-1500_20MIN.m Operator Demo User

Sample Name $0616 \quad$ Instrumen compact

8255754.2017

Comment

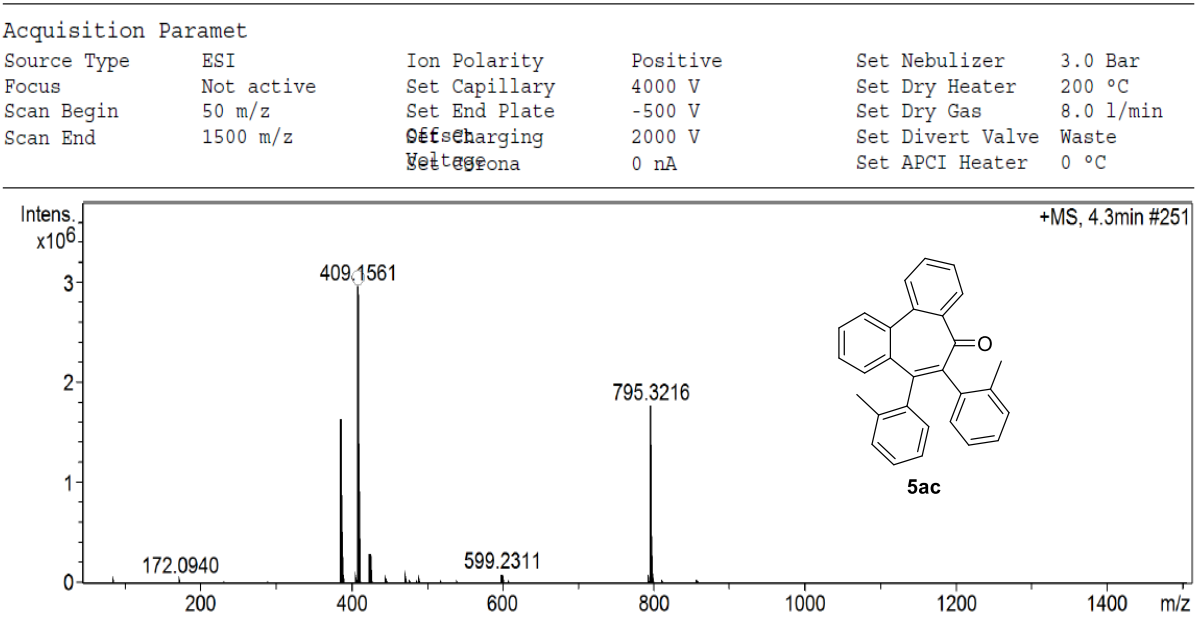

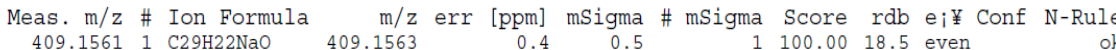
$409.1561 \quad 1 \mathrm{C} 29 \mathrm{H} 22 \mathrm{NaO}$ 


\section{Mass Spectrum SmartFormula Report}

Analysis Info

Acquisition D 2021-06-18 21:14:23

Analysis Name F: \GAO FEN BIAN(YANG LING YUN) \GAOFENBIAN-2021-06-16\0616_RD3_01_6572.d

Method LC_NO UV_P50-1500_20MIN.m

Operator Demo User

Sample Name 0616

Instrumen compact

8255754.2017

Comment

Acquisition Paramet

Source Type ESI

Focus Not active

Scan Begin $\quad 50 \mathrm{~m} / \mathrm{z}$

Scan End $\quad 1500 \mathrm{~m} / \mathrm{z}$

$\begin{array}{ll}\text { Ion Polarity } & \text { Positive } \\ \text { Set Capillary } & 4000 \mathrm{~V} \\ \text { Set End Plate } & -500 \mathrm{~V} \\ \text { Oef sebarging } & 2000 \mathrm{~V} \\ \text { gettegfona } & 0 \mathrm{nA}\end{array}$

Set Nebulizer $\quad 3.0$ Bar

Jettagena $0 \mathrm{nA}$

Valve Waste

Intens. + +MS, $3.5 \mathrm{~min} \# 204$

$\times 10^{5}$

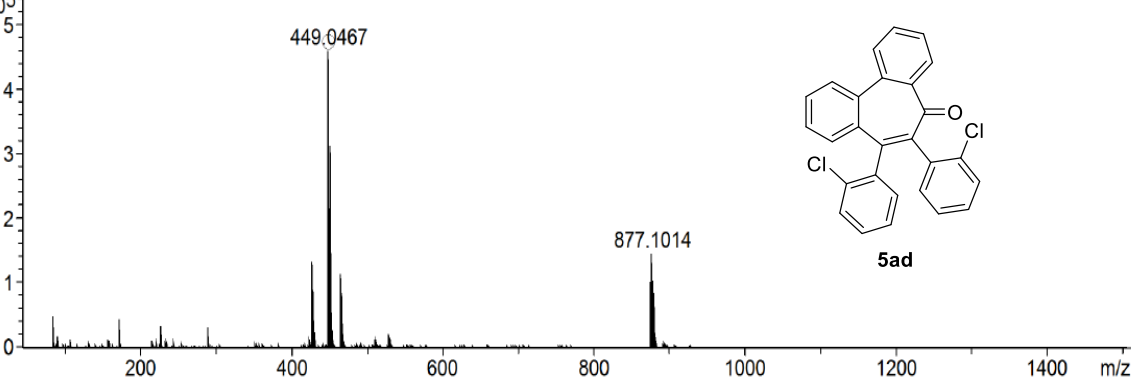

Meas. $\mathrm{m} / \mathrm{z}$ \# Ion Formula

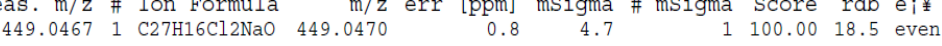

/ $z$ err [ppm] mSigma \# mSigma Score rdb ei¥ Conf $\mathrm{N}$-Rule

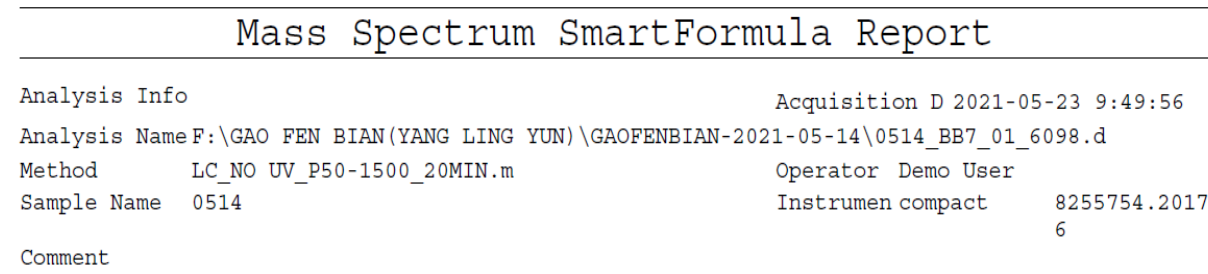

Comment

\begin{tabular}{llllll}
\hline Acquisition & Paramet & & & & \\
Source Type & ESI & Ion Polarity & Positive & Set Nebulizer & $3.0 \mathrm{Bar}$ \\
Focus & Not active & Set Capillary & $4000 \mathrm{~V}$ & Set Dry Heater & $200^{\circ} \mathrm{C}$ \\
Scan Begin & $50 \mathrm{~m} / \mathrm{z}$ & Set End Plate & $-500 \mathrm{~V}$ & Set Dry Gas & $8.01 / \mathrm{min}$ \\
Scan End & $1500 \mathrm{~m} / \mathrm{z}$ & Oefsebarging & $2000 \mathrm{~V}$ & Set Divert Valve Waste \\
& & SeEtegrona & $0 \mathrm{nA}$ & Set APCI Heater $0{ }^{\circ} \mathrm{C}$ \\
\hline
\end{tabular}

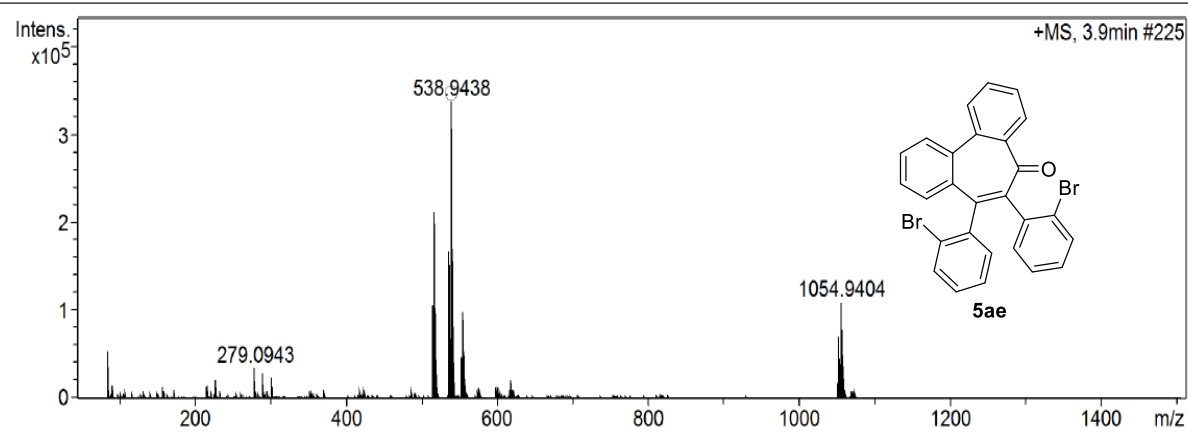

Meas. $\mathrm{m} / \mathrm{z}$ \# Ion Formula $\mathrm{m} / \mathrm{z}$ err [ppm] mSigma \# mSigma Score rdb ei¥ Conf $\mathrm{N}-\mathrm{Rule}$

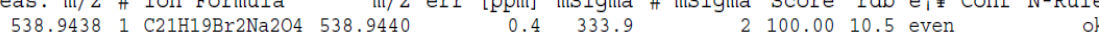




\section{Mass Spectrum SmartFormula Report}

Analysis Info

Acquisition D 2021-06-18 19:28:02

Analysis Name F:\GAO FEN BIAN(YANG LING YUN) \GAOFENBIAN-2021-06-16\0616_RC6_01_6567.d

Method LC_NO UV_P50-1500_20MIN.m Operator Demo User

Sample Name 0616 Instrumen compact

8255754.2017

Comment

\begin{tabular}{llllll}
\hline Acquisition & Paramet & & & & \\
Source Type & ESI & Ion Polarity & Positive & Set Nebulizer & $3.0 \mathrm{Bar}$ \\
Focus & Not active & Set Capillary & $4000 \mathrm{~V}$ & Set Dry Heater & $200^{\circ} \mathrm{C}$ \\
Scan Begin & $50 \mathrm{~m} / \mathrm{z}$ & Set End Plate & $-500 \mathrm{~V}$ & Set Dry Gas & $8.0 \mathrm{l} / \mathrm{min}$ \\
Scan End & $1500 \mathrm{~m} / \mathrm{z}$ & Oefétharging & $2000 \mathrm{~V}$ & Set Divert Valve Waste \\
& & Settegrona & $0 \mathrm{nA}$ & Set APCI Heater $0{ }^{\circ} \mathrm{C}$
\end{tabular}

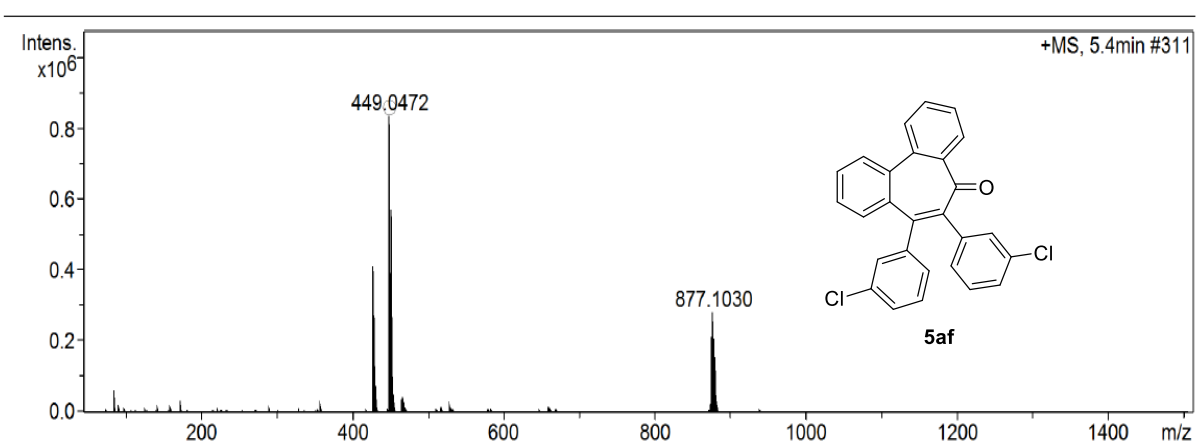

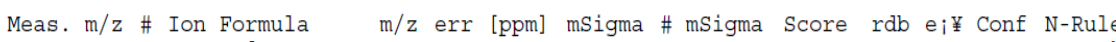

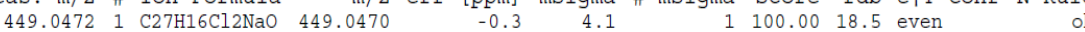

\section{Mass Spectrum SmartFormula Report}

Analysis Info

Acquisition D 2021-05-23 8:03:33

Analysis Name F:\GAO FEN BIAN(YANG LING YUN) \GAOFENBIAN-2021-05-14\0514_BB2_01_6093.d

Method LC_NO UV_P50-1500_20MIN.m Operator Demo User

Comment

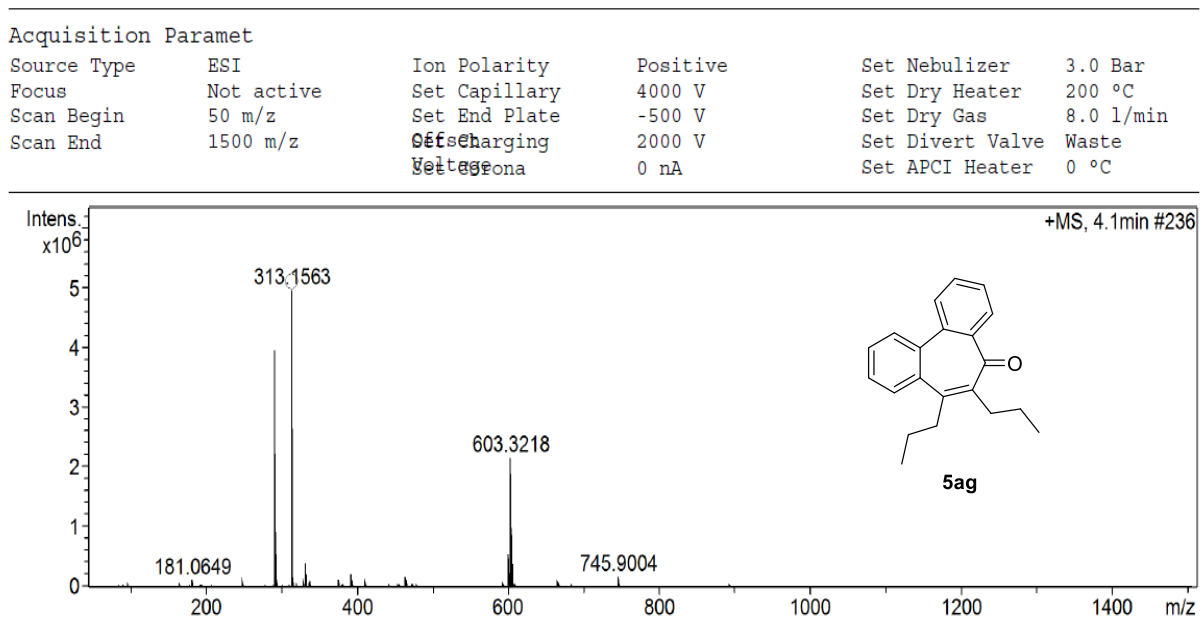

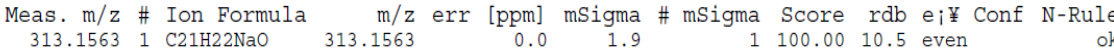
$313.1563 \quad 1$ C21H20170 313.1563 


\section{Mass Spectrum SmartFormula Report}

Analysis Info

Acquisition D 2021-01-28 4:21:21

Analysis Name F:\GAOFENBIAN-2021-1-29\0125_BA8_01_4025.d

Method

LC_NO UV_P50-1500_6MIN.m

Operator Demo User

Sample Name 0125

Instrumen compact

8255754.2017

Comment

\begin{tabular}{llllll}
\hline Acquisition & Paramet & & & \\
Source Type & ESI & Ion Polarity & Positive & Set Nebulizer & $3.0 \mathrm{Bar}$ \\
Focus & Not active & Set Capillary & $4000 \mathrm{~V}$ & Set Dry Heater & $200^{\circ} \mathrm{C}$ \\
Scan Begin & $50 \mathrm{~m} / \mathrm{z}$ & Set End Plate & $-500 \mathrm{~V}$ & Set Dry Gas & $8.0 \mathrm{l} / \mathrm{min}$ \\
Scan End & $1500 \mathrm{~m} / \mathrm{z}$ & OEf & Setharging & $2000 \mathrm{~V}$ & Set Divert Valve Waste \\
& & Setegena & $0 \mathrm{nA}$ & Set APCI Heater $0{ }^{\circ} \mathrm{C}$
\end{tabular}

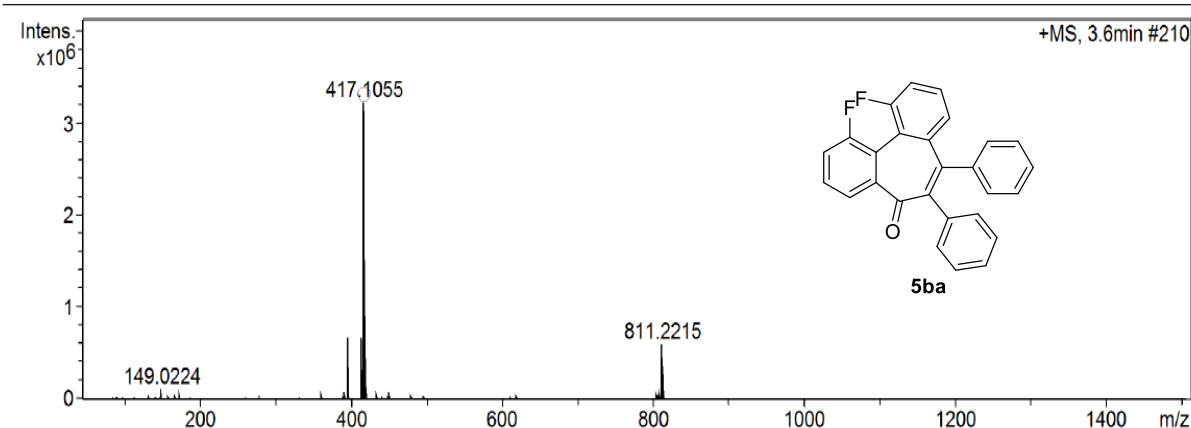

Meas. $\mathrm{m} / \mathrm{z}$ \# Ion Formula $\mathrm{m} / \mathrm{z}$ err [ppm] mSigma \# mSigma Score $\mathrm{rdb}$ e $¥$ $¥$ Conf $\mathrm{N}-\mathrm{Rule}$

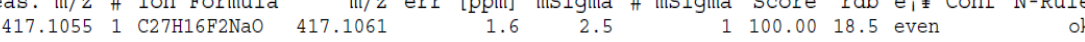

\section{Mass Spectrum SmartFormula Report}

Analysis Info

Acquisition D 2021-01-28 5:36:41

Analysis Name F: \GAOFENBIAN-2021-1-29\0125_BC2_01_4035.d

Method

LC_NO UV_P50-1500_6MIN.m

Operator Demo User

Instrumen compact

8255754.2017

Sample Name 0125

Comment

$\begin{array}{llllll}\text { Acquisition } & \text { Paramet } & & & \\ \text { Source Type } & \text { ESI } & \text { Ion Polarity } & \text { Positive } & \text { Set Nebulizer } & 3.0 \mathrm{Bar} \\ \text { Focus } & \text { Not active } & \text { Set Capillary } & 4000 \mathrm{~V} & \text { Set Dry Heater } & 200^{\circ} \mathrm{C} \\ \text { Scan Begin } & 50 \mathrm{~m} / \mathrm{z} & \text { Set End Plate } & -500 \mathrm{~V} & \text { Set Dry Gas } & 8.01 / \mathrm{min} \\ \text { Scan End } & 1500 \mathrm{~m} / \mathrm{z} & \text { Sefsebarging } & 2000 \mathrm{~V} & \text { Set Divert Valve Waste } \\ & & \text { Gettegena } & 0 \mathrm{nA} & \text { Set APCI Heater } 00^{\circ} \mathrm{C}\end{array}$

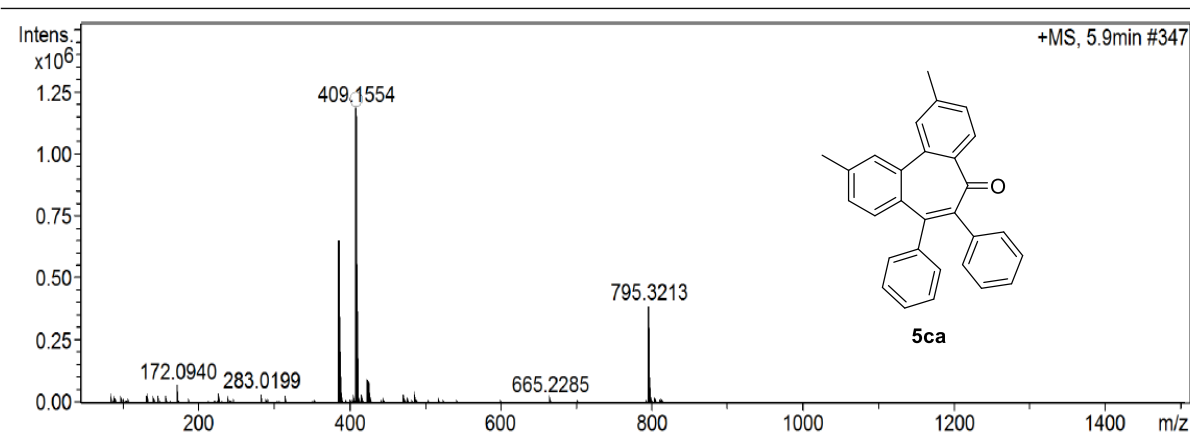

Meas. $\mathrm{m} / \mathrm{z}$ \# Ion Formula $409.1554 \quad 1$ C29H $22 \mathrm{NaO}$

$\mathrm{m} / \mathrm{z}$ err [ppm] mSigma \# mSigma Score rdb ei¥ Conf N-Rule

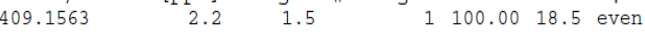




\section{Mass Spectrum SmartFormula Report}

Analysis Info

Analysis Name F: \GAOFENBIAN-2021-1-29\0125_BA4_01_4021.d

Method

LC NO UV P50-1500 6MIN.m

Sample Name $01 \overline{25}$

Comment
Acquisition D 2021-01-28 $3: 51: 42$

Operator Demo User

Instrumen compact

8255754.2017

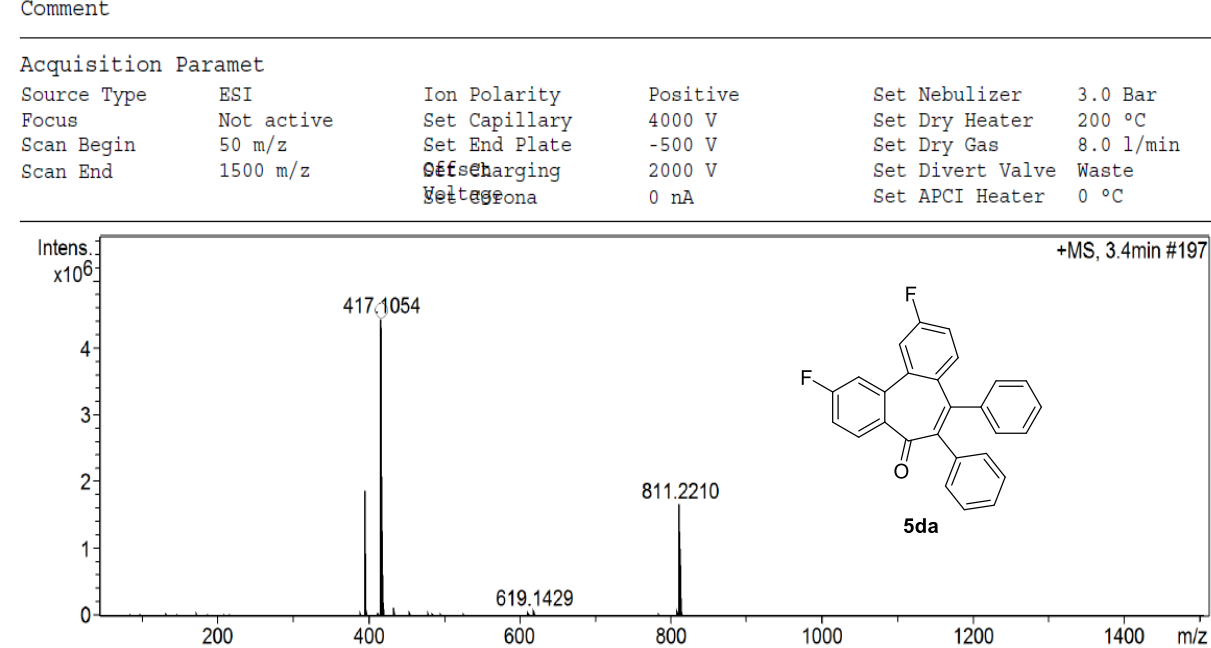

Meas. $\mathrm{m} / \mathrm{z}$ \# Ion Formula

$\mathrm{m} / \mathrm{z}$ err [ppm] mSigma \# mSigma Score $r d b$ e $¥ ¥$ Conf $\mathrm{N}$-Rule 417.1061

$1.7 \quad 0.7$

1100.0018 .5 even

\section{Mass Spectrum SmartFormula Report}

Analysis Info

Acquisition D 2021-01-28 3:59:19

Analysis Name F:\GAOFENBIAN-2021-1-29\0125_BA5_01_4022.d

Method

LC_NO UV_P50-1500_6MIN.m

Operator Demo User

Sample Name 0125

Instrumen compact

8255754.2017

Comment

Acquisition Paramet

\begin{tabular}{|c|c|c|c|c|c|}
\hline Source Type & ESI & Ion Polarity & Positive & Set Nebulizer & $3.0 \mathrm{Bar}$ \\
\hline Focus & Not active & Set Capillary & $4000 \mathrm{~V}$ & Set Dry Heater & $200^{\circ} \mathrm{C}$ \\
\hline Scan Begin & $50 \mathrm{~m} / \mathrm{z}$ & Set End Plate & $-500 \mathrm{~V}$ & Set Dry Gas & $8.01 / \mathrm{min}$ \\
\hline Scan End & $1500 \mathrm{~m} / \mathrm{z}$ & $\begin{array}{l}\text { offsebarging } \\
\text { dettageona }\end{array}$ & $2000 \mathrm{~V}$ & $\begin{array}{l}\text { Set Divert Valve } \\
\text { Set APCI Heater }\end{array}$ & $\begin{array}{l}\text { Waste } \\
0{ }^{\circ} \mathrm{C}\end{array}$ \\
\hline
\end{tabular}

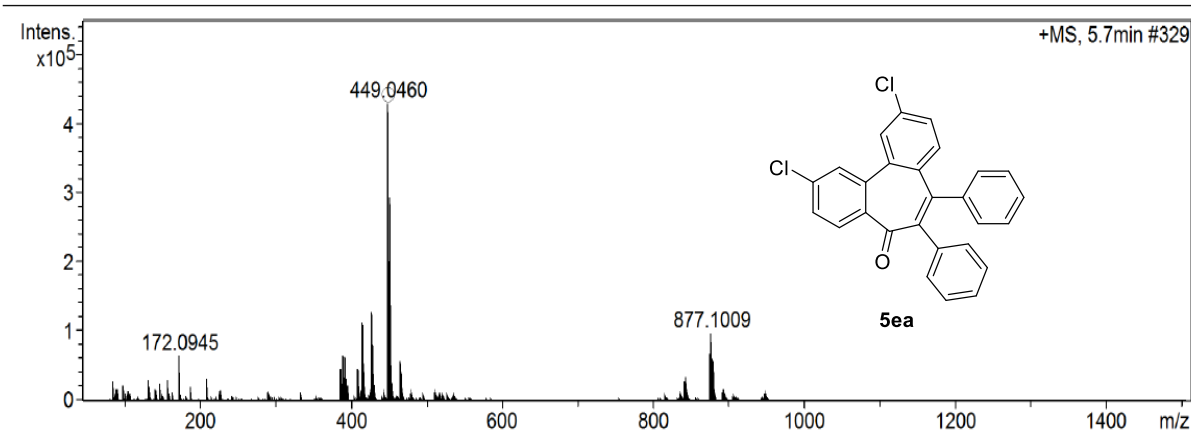

Meas. $\mathrm{m} / \mathrm{z}$ \# Ion Formul

$\mathrm{m} / \mathrm{z}$ err [ppm] mSigma \# mSigma Score rdb e $¥ ¥$ Conf $\mathrm{N}$-Rule $449.0460 \quad 1$ C27H16Cl2NaO 449.0470

$2.3 \quad 4.1$

100.0018 .5 even 


\section{Mass Spectrum SmartFormula Report}

Analysis Info

Acquisition D 2021-05-23 4:29:50

Analysis Name F:\GAO FEN BIAN (YANG LING YUN) \GAOFENBIAN-2021-05-14\0514_GE8_01_6083.d

Method LC NO UV P50-1500 20MIN.m

Operator Demo User

Sample Name $0 \overline{5} \overline{14}$

Instrumen compact

8255754.2017

Comment

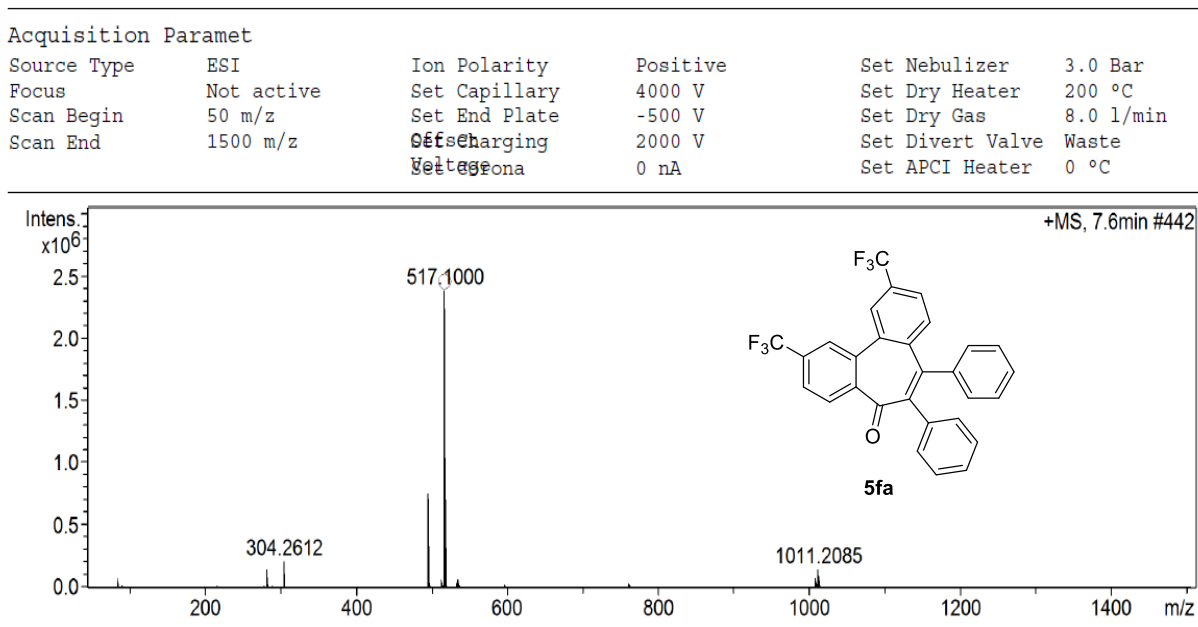

Meas. m/z \# Ion Formula $\mathrm{m} / \mathrm{z}$ err [ppm] mSigma \# mSigma Score $\mathrm{rdb}$ ej¥ Conf $\mathrm{N}$-Rule

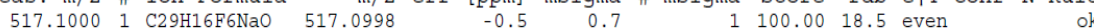

\section{Mass Spectrum SmartFormula Report}

Analysis Info

Acquisition D 2021-05-23 8:24:50

Analysis Name F:\GAO FEN BIAN(YANG LING YUN) \GAOFENBIAN-2021-05-14\0514_BB3_01_6094.d

Method LC_NO UV_P50-1500_20MIN.m Operator Demo User

Sample Name $0514 \quad$ Instrumen compact

8255754.2017

Comment

\begin{tabular}{llllll}
\hline Acquisition & Paramet & & & \\
Source Type & ESI & Ion Polarity & Positive & Set Nebulizer & 3.0 Bar \\
Focus & Not active & Set Capillary & $4000 \mathrm{~V}$ & Set Dry Heater & $200^{\circ} \mathrm{C}$ \\
Scan Begin & $50 \mathrm{~m} / \mathrm{z}$ & Set End Plate & $-500 \mathrm{~V}$ & Set Dry Gas & $8.0 \mathrm{I} / \mathrm{min}$ \\
Scan End & $1500 \mathrm{~m} / \mathrm{z}$ & OEf & Setharging & $2000 \mathrm{~V}$ & Set Divert Valve Waste \\
& & Settergana & $0 \mathrm{nA}$ & Set APCI Heater $0{ }^{\circ} \mathrm{C}$
\end{tabular}

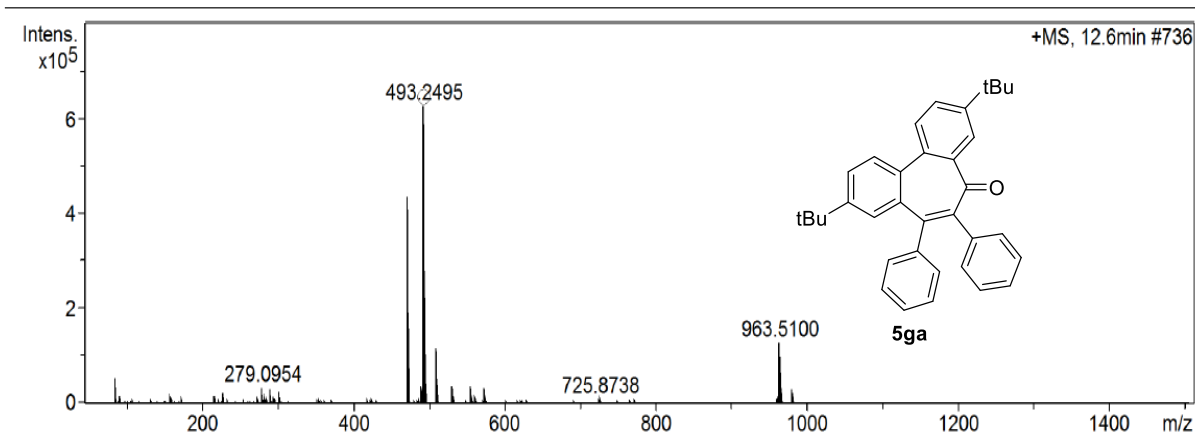

Meas. $\mathrm{m} / \mathrm{z}$ \# Ion Formula

$\mathrm{m} / \mathrm{z}$ err [ppm] mSigma \# mSigma Score rdb ei¥ Conf $\mathrm{N}-\mathrm{Rule}$ $493.2495 \quad 1 \quad \mathrm{C} 35 \mathrm{H} 34 \mathrm{NaO}$

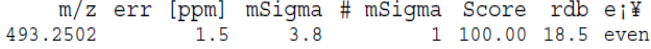




\section{Mass Spectrum SmartFormula Report}

Analysis Info

Acquisition D 2021-01-28 6:21:52

Analysis Name F: \GAOFENBIAN-2021-1-29\0125_BC8_01_4041.d

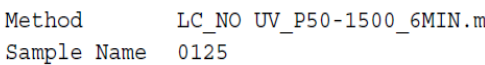

Method

LC NO UV P50-1500 6MIN.m

Sample Name 0125

Operator Demo Use

Inst rumen compact

8255754.2017

Comment

$\begin{array}{llllll}\text { Acquisition } & \text { Paramet } & & & \\ \text { Source Type } & \text { ESI } & \text { Ion Polarity } & \text { Positive } & \text { Set Nebulizer } & 3.0 \text { Bar } \\ \text { Focus } & \text { Not active } & \text { Set Capillary } & 4000 \mathrm{~V} & \text { Set Dry Heater } & 200^{\circ} \mathrm{C} \\ \text { Scan Begin } & 50 \mathrm{~m} / \mathrm{z} & \text { Set End Plate } & -500 \mathrm{~V} & \text { Set Dry Gas } & 8.0 \mathrm{l} / \mathrm{min} \\ \text { Scan End } & 1500 \mathrm{~m} / \mathrm{z} & \text { OEf } & \text { Setharging } & 2000 \mathrm{~V} & \text { Set Divert Valve Waste } \\ & & \text { Setteg甲ona } & 0 \mathrm{nA} & \text { Set APCI Heater } 0{ }^{\circ} \mathrm{C}\end{array}$

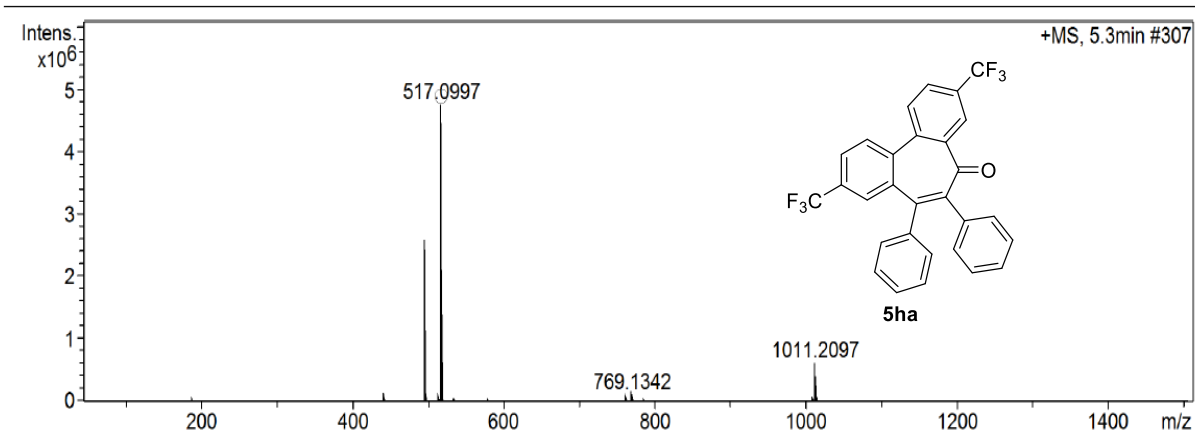

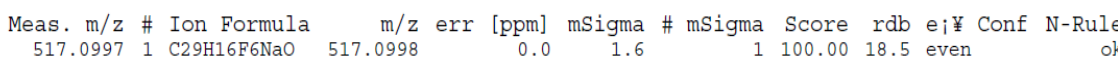

\section{Mass Spectrum SmartFormula Report}

Analysis Info

Acquisition D 2021-01-28 5:51:56

Analysis Name F:\GAO FEN BIAN(YANG LING YUN) \GAOFENBIAN-2021-1-29\0125 BC4 01 4037.d

Method LC_NO UV_P50-1500_6MIN.m Operator Demo User

Sample Name $0125 \quad$ Instrumen compact

8255754.2017

Comment

Acquisition Paramet

\begin{tabular}{llllll} 
Source Type & ESI & Ion Polarity & Positive & Set Nebulizer & $3.0 \mathrm{Bar}$ \\
Focus & Not active & Set Capillary & $4000 \mathrm{~V}$ & Set Dry Heater & $200^{\circ} \mathrm{C}$ \\
Scan Begin & $50 \mathrm{~m} / \mathrm{z}$ & Set End Plate & $-500 \mathrm{~V}$ & Set Dry Gas & $8.0 \mathrm{l} / \mathrm{min}$ \\
Scan End & $1500 \mathrm{~m} / \mathrm{z}$ & Offetsebarging & $2000 \mathrm{~V}$ & Set Divert Valve Waste \\
& & Settegena & $0 \mathrm{nA}$ & Set APCI Heater & $0^{\circ} \mathrm{C}$ \\
\hline
\end{tabular}

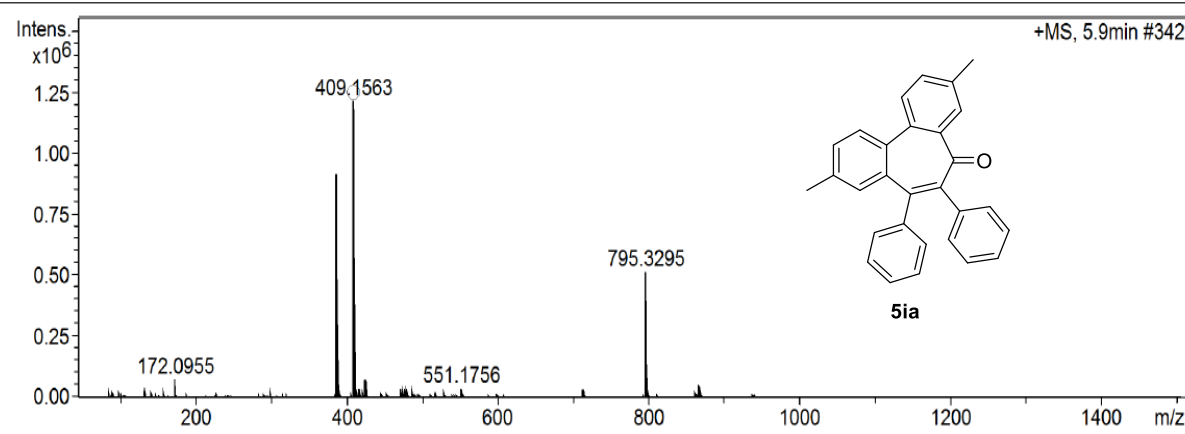

Meas. $\mathrm{m} / \mathrm{z}$ \# Ion Formula

$\mathrm{m} / \mathrm{z}$ err [ppm] mSigma \# mSigma Score rdb e ¡¥ Conf $\mathrm{N}$-Rule

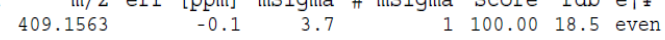




\section{Mass Spectrum SmartFormula Report}

Analysis Info

Acquisition D 2021-01-28 4:28:42

Analysis Name F: \GAOFENBIAN-2021-1-29\0125_BB1_01_4026.d LC NO UV P50-1500 6MIN.m

Operator Demo User

Sample Name 0125

Instrumen compact

8255754.2017

Comment

$\begin{array}{llllll}\text { Acquisition } & \text { Paramet } & & & \\ \text { Source Type } & \text { ESI } & \text { Ion Polarity } & \text { Positive } & \text { Set Nebulizer } & 3.0 \mathrm{Bar} \\ \text { Focus } & \text { Not active } & \text { Set Capillary } & 4000 \mathrm{~V} & \text { Set Dry Heater } & 200^{\circ} \mathrm{C} \\ \text { Scan Begin } & 50 \mathrm{~m} / \mathrm{z} & \text { Set End Plate } & -500 \mathrm{~V} & \text { Set Dry Gas } & 8.01 / \mathrm{min} \\ \text { Scan End } & 1500 \mathrm{~m} / \mathrm{z} & \text { Oefsetarging } & 2000 \mathrm{~V} & \text { Set Divert Valve Waste } \\ & & \text { Settegena } & 0 \mathrm{nA} & \text { Set APCI Heater } 0{ }^{\circ} \mathrm{C}\end{array}$

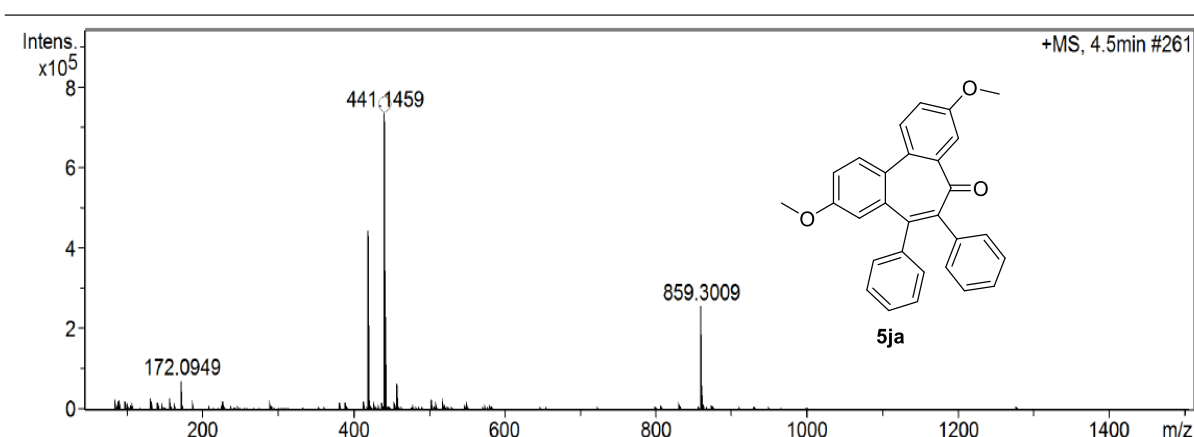

Meas. $\mathrm{m} / \mathrm{z}$ \# Ion Formul $441.14591 \mathrm{C} 29 \mathrm{H} 22 \mathrm{NaO} 3$

$\mathrm{m} / \mathrm{z}$ err [ppm] mSigma \# mSigma score rdb e $¥ ¥$ Conf $\mathrm{N}$-Rule $441.1461 \quad 0.5 \quad 2.5 \quad 1100.0018 .5$ even

\section{Mass Spectrum SmartFormula Report}

Analysis Info

Acquisition D 2021-05-23 7:42:17

Analysis Name F:\GAO FEN BIAN(YANG LING YUN) \GAOFENBIAN-2021-05-14\0514_BB1_01_6092.d

Method LC_NO UV_P50-1500_20MIN.m

Operator Demo User

Sample Name 0514

Instrumen compact

8255754.2017

Comment

Acquisition Paramet

\begin{tabular}{|c|c|c|c|c|c|}
\hline Source Type & ESI & Ion Polarity & Positive & Set Nebulizer & $3.0 \mathrm{Bar}$ \\
\hline Focus & Not active & Set Capillary & $4000 \mathrm{~V}$ & Set Dry Heater & $200{ }^{\circ} \mathrm{C}$ \\
\hline Scan Begin & $50 \mathrm{~m} / \mathrm{z}$ & Set End Plate & $-500 \mathrm{~V}$ & Set Dry Gas & $8.0 \mathrm{l} / \mathrm{min}$ \\
\hline Scan End & $1500 \mathrm{~m} / \mathrm{z}$ & $\begin{array}{l}\text { Oefsebarging } \\
\text { gettegeona }\end{array}$ & $\begin{array}{l}2000 \mathrm{~V} \\
0 \mathrm{nA}\end{array}$ & $\begin{array}{l}\text { Set Divert Valve } \\
\text { Set APCI Heater }\end{array}$ & $\begin{array}{l}\text { Waste } \\
0{ }^{\circ} \mathrm{C}\end{array}$ \\
\hline
\end{tabular}

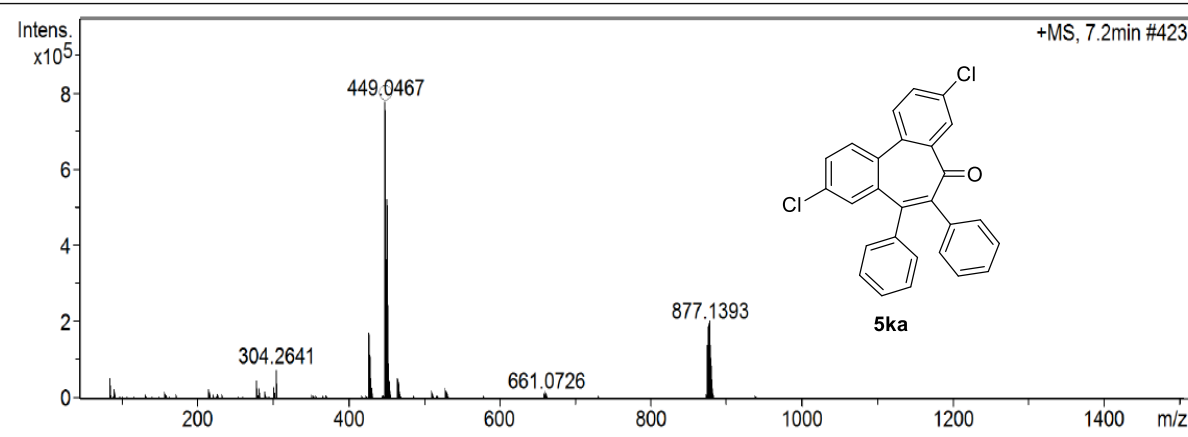

Meas. $\mathrm{m} / \mathrm{z}$ \# Ion Formula $\mathrm{m} / \mathrm{z}$ err [ppm] mSigma \# mSigma Score rdb ei¥ Conf $\mathrm{N}-\mathrm{Rul}$

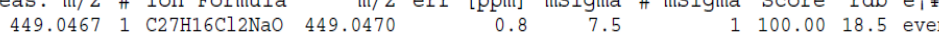




\section{Mass Spectrum SmartFormula Report}

Analysis Info

Acquisition D 2021-06-18 21:57:59

Analysis Name F:\GAO FEN BIAN(YANG LING YUN) \GAOFENBIAN-2021-06-16\0616_RD5_01_6574.d

Method LC_NO UV_P50-1500_20MIN.m Operator Demo User

Sample Name 0616 Instrumen compact

8255754.2017

Comment

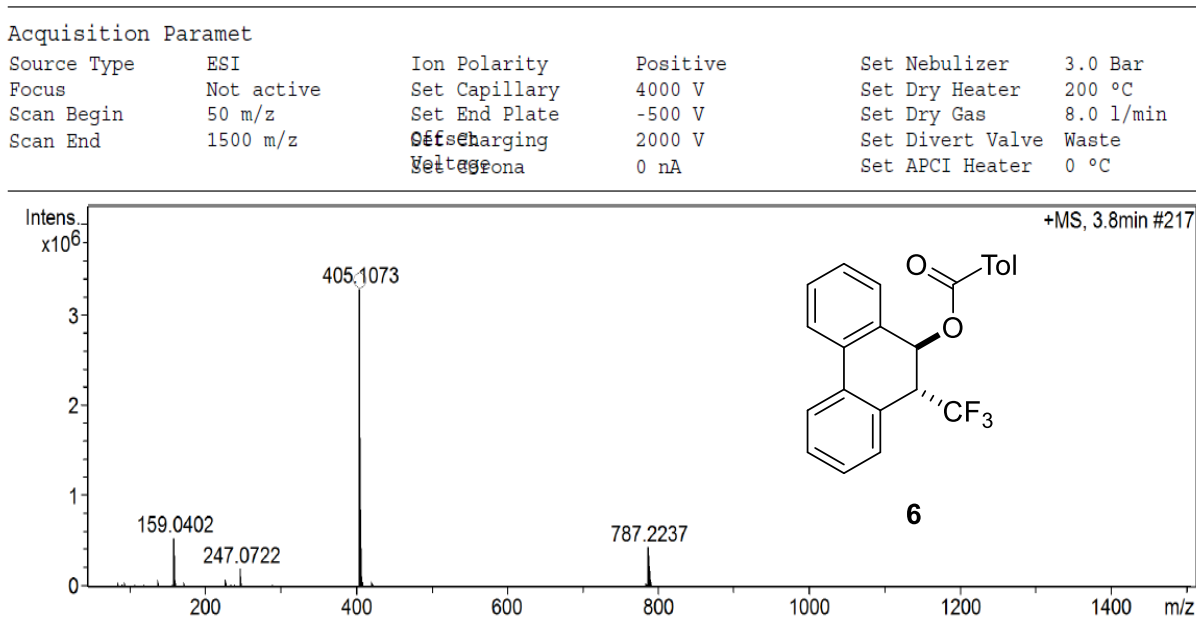

Meas. $\mathrm{m} / \mathrm{z}$ \# Ion Formula $\mathrm{m} / \mathrm{z}$ err [ppm] mSigma \# mSigma Score rdb ei¥ Conf N-Rule $405.1073 \quad 1 \mathrm{C} 23 \mathrm{H} 17 \mathrm{~F} 3 \mathrm{NaO} 2 \quad 405.1073$

\section{Mass Spectrum SmartFormula Report}

Analysis Info

Acquisition D 2021-05-16 9:30:32

Analysis Name F:\GAO FEN BIAN(YANG LING YUN) \GAOFENBIAN-2021-05-14\0514_RB7_01_6013.d

Method LC_NO UV_P50-1500_20MIN.m

Operator Demo User

Sample Name 0514

Instrumen compact

8255754.2017

Comment

Acquisition Paramet

\begin{tabular}{llllll} 
Source Type & ESI & Ion Polarity & Positive & Set Nebulizer & 3.0 Bar \\
Focus & Not active & Set Capillary & $4000 \mathrm{~V}$ & Set Dry Heater & $2000^{\circ} \mathrm{C}$ \\
Scan Begin & $50 \mathrm{~m} / \mathrm{z}$ & Set End Plate & $-500 \mathrm{~V}$ & Set Dry Gas & $8.0 \mathrm{l} / \mathrm{min}$ \\
Scan End & $1500 \mathrm{~m} / \mathrm{z}$ & OEf & Getharging & $2000 \mathrm{~V}$ & Set Divert Valve Waste \\
& & Settegrona & $0 \mathrm{nA}$ & Set APCI Heater $00^{\circ} \mathrm{C}$ \\
\hline
\end{tabular}

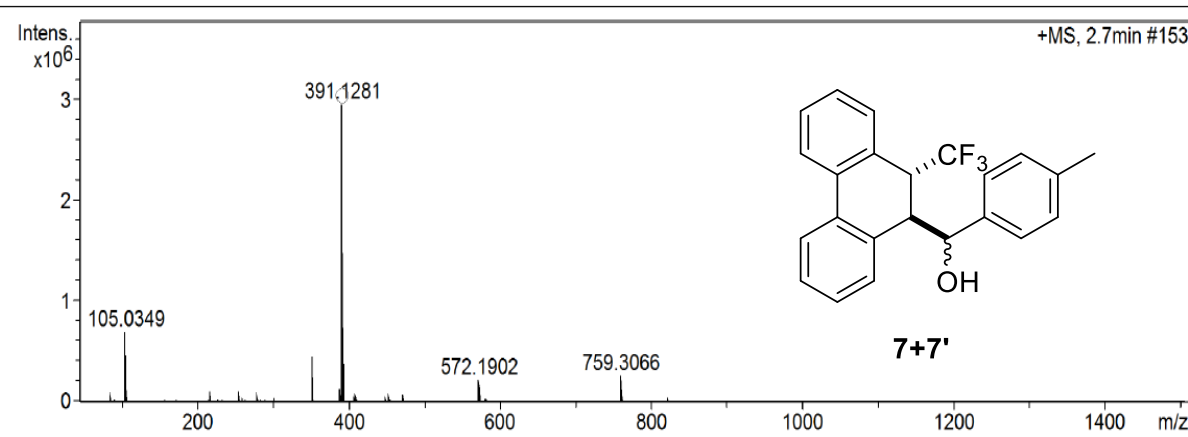

Meas. $\mathrm{m} / \mathrm{z}$ \# Ion Formula $\mathrm{m} / \mathrm{z}$ err [ppm] mSigma \# mSigma Score rdb ei¥ Conf $\mathrm{N}$-Rule 


\section{Mass Spectrum SmartFormula Report}

Analysis Info

Acquisition D 2021-06-24 13:39:15

Analysis Name F:\GAO FEN BIAN(YANG LING YUN) \0624_RC1_01_6643.d

Method LC_NO UV_P50-1500_20MIN.m Operator Demo User

Sample Name 0624 Instrumen compact

8255754.2017

Comment

\begin{tabular}{llllll}
\hline Acquisition & Paramet & & & \\
Source Type & ESI & Ion Polarity & Positive & Set Nebulizer & $3.0 \mathrm{Bar}$ \\
Focus & Not active & Set Capillary & $4000 \mathrm{~V}$ & Set Dry Heater $200^{\circ} \mathrm{C}$ \\
Scan Begin & $50 \mathrm{~m} / \mathrm{z}$ & Set End Plate & $-500 \mathrm{~V}$ & Set Dry Gas & $8.01 / \mathrm{min}$ \\
Scan End & $1500 \mathrm{~m} / \mathrm{z}$ & OEf & Setharging & $2000 \mathrm{~V}$ & Set Divert Valve Waste \\
& & Settena & $0 \mathrm{nA}$ & Set APCI Heater $0{ }^{\circ} \mathrm{C}$ \\
\hline
\end{tabular}

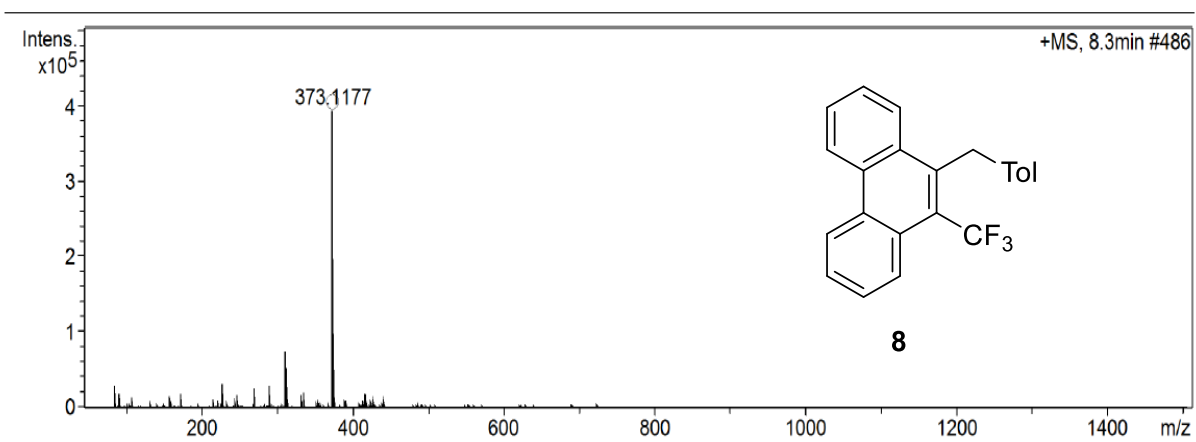

Meas. $\mathrm{m} / \mathrm{z}$ \# Ion Formula $\mathrm{m} / \mathrm{z}$ err [ppm] mSigma \# mSigma Score $\mathrm{rdb}$ e $¥$ $¥$ Conf $\mathrm{N}-\mathrm{Rule}$

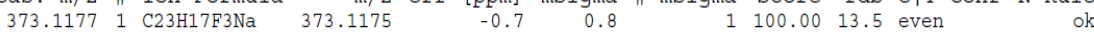

\section{Mass Spectrum SmartFormula Report}

Analysis Info

Acquisition D 2021-06-18 22:19:46

Analysis Name F:\GAO FEN BIAN(YANG LING YUN) \GAOFENBIAN-2021-06-16\0616_RD6_01_6575.d

Method LC_NO UV_P50-1500_20MIN.m Operator Demo User

Sample Name $0616 \quad$ Instrumen compact

8255754.2017

Comment

Acquisition Paramet

\begin{tabular}{llllll} 
Source Type & ESI & Ion Polarity & Positive & Set Nebulizer & 3.0 Bar \\
Focus & Not active & Set Capillary & $4000 \mathrm{~V}$ & Set Dry Heater & $200^{\circ} \mathrm{C}$ \\
Scan Begin & $50 \mathrm{~m} / \mathrm{z}$ & Set End Plate & $-500 \mathrm{~V}$ & Set Dry Gas & $8.0 \mathrm{l} / \mathrm{min}$ \\
Scan End & $1500 \mathrm{~m} / \mathrm{z}$ & Offseharging & $2000 \mathrm{~V}$ & Set Divert Valve Waste \\
& & Se\#tagena & $0 \mathrm{nA}$ & Set APCI Heater $0{ }^{\circ} \mathrm{C}$ \\
\hline
\end{tabular}

Intens: + +MS, $5.2 \mathrm{~min} \# 305$

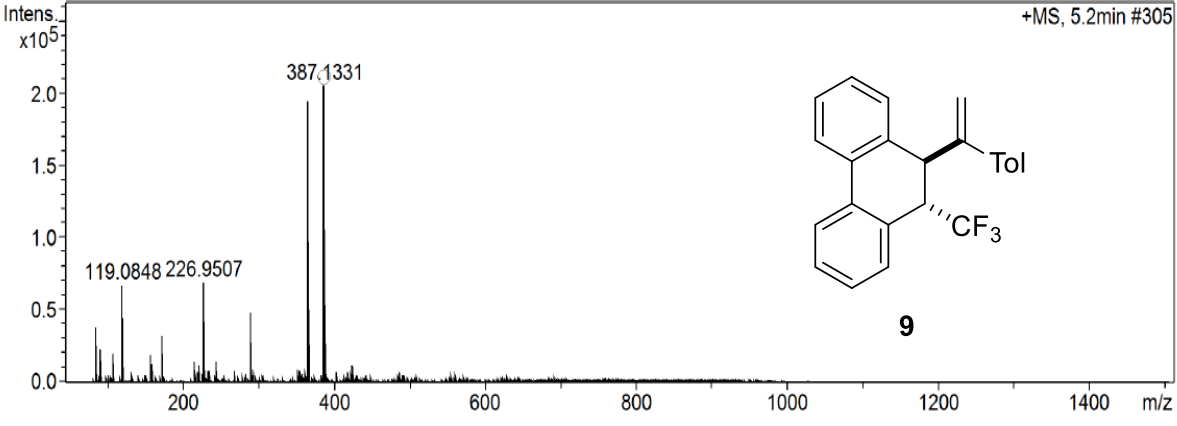

Meas. $\mathrm{m} / \mathrm{z}$ \# Ion Formul 387.1331 1 $224 \mathrm{H} 19 \mathrm{~F} 3 \mathrm{Na}$

$\mathrm{m} / \mathrm{z}$ err [ppm] mSigma \# mSigma Score rdb ei¥ Conf N-Rule

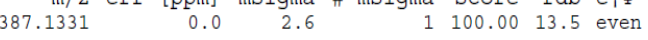




\section{Mass Spectrum SmartFormula Report}

Analysis Info

Acquisition D 2021-06-18 20:10:33

Analysis Name F: \GAO FEN BIAN(YANG LING YUN) \GAOFENBIAN-2021-06-16\0616_RC8_01_6569.d

Method LC_NO UV_P50-1500_20MIN.m Operator Demo User

Sample Name $06 \overline{16} \quad$ Instrumen compact

Comment

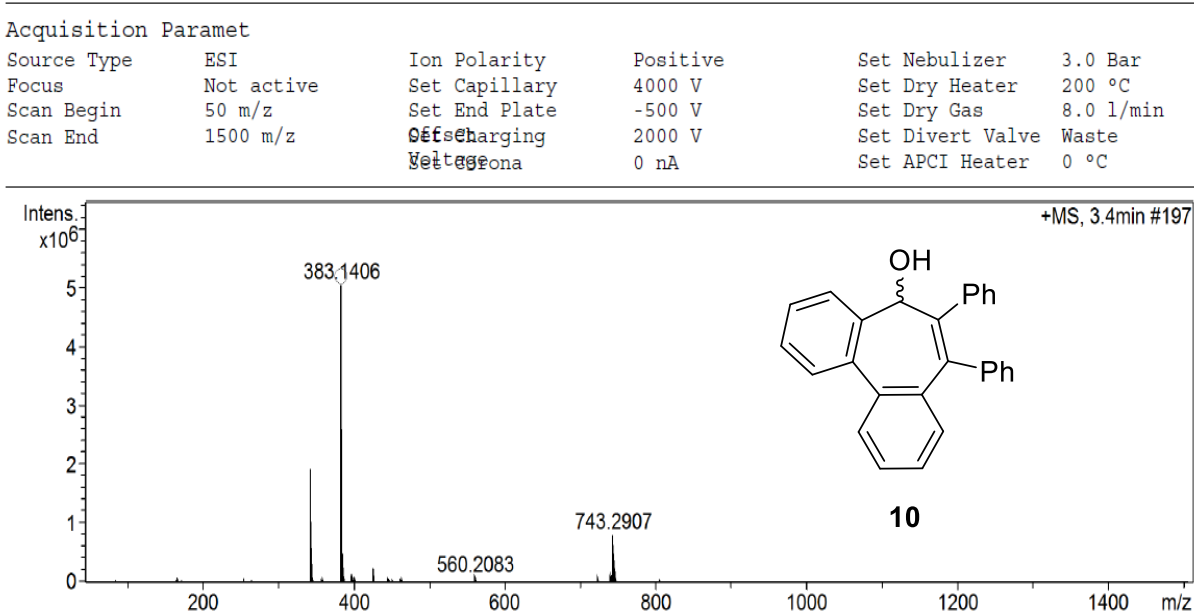

Meas. $\mathrm{m} / \mathrm{z}$ \# Ion Formula $\mathrm{m} / \mathrm{z}$ err [ppm] mSigma \# mSigma Score rdb ei¥ Conf $\mathrm{N}$-Rule $383.1406 \quad 1 \mathrm{C} 27 \mathrm{H} 20 \mathrm{NaO}$

\section{Mass Spectrum SmartFormula Report}

Analysis Info

Acquisition D 2021-06-18 20:31:49

Analysis Name F:\GAO FEN BIAN(YANG LING YUN) \GAOFENBIAN-2021-06-16\0616_RD1_01_6570.d

Method

LC NO UV P50-1500 20MIN.m

Operator Demo User

Sample Name 0616

Inst rumen compact

Comment

\begin{tabular}{llllll}
\hline Acquisition & Paramet & & & & \\
Source Type & ESI & Ion Polarity & Positive & Set Nebulizer & $3.0 \mathrm{Bar}$ \\
Focus & Not active & Set Capillary & $4000 \mathrm{~V}$ & Set Dry Heater & $200^{\circ} \mathrm{C}$ \\
Scan Begin & $50 \mathrm{~m} / \mathrm{z}$ & Set End Plate & $-500 \mathrm{~V}$ & Set Dry Gas & $8.01 / \mathrm{min}$ \\
Scan End & $1500 \mathrm{~m} / \mathrm{z}$ & Oefsebarging & $2000 \mathrm{~V}$ & Set Divert Valve Waste \\
& & SeEtegrona & $0 \mathrm{nA}$ & Set APCI Heater $00^{\circ} \mathrm{C}$ \\
\hline
\end{tabular}

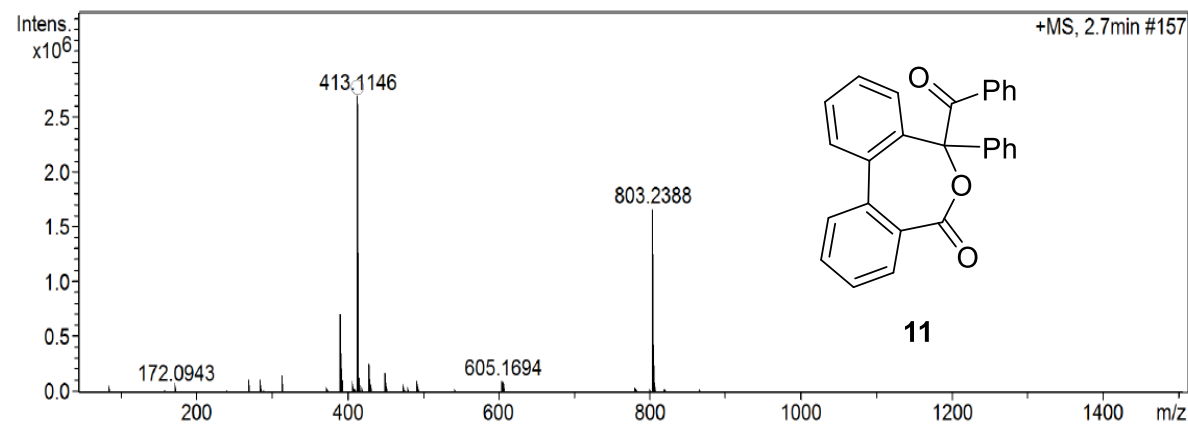

Meas. $\mathrm{m} / \mathrm{z}$ \# Ion Formula

$\mathrm{m} / \mathrm{z}$ err [ppm] mSigma \# mSigma Score rdb e $¥$ ¥ Conf $\mathrm{N}$-Rule $413.1146 \quad 1$ C27H18NaO3 413.1148

0.5 igma

$100.00 \quad 18.5$ even 


\section{Mass Spectrum SmartFormula Report}

Analysis Info

Acquisition D 2021-05-16 10:34:20

Analysis Name F: $\backslash 0514 \_R C 2 \_01 \_6016$.d

Method LC_NO UV_P50-1500_20MIN.m

Sample Name 0514

Operator Demo User

Instrumen compact

8255754.2017

Comment

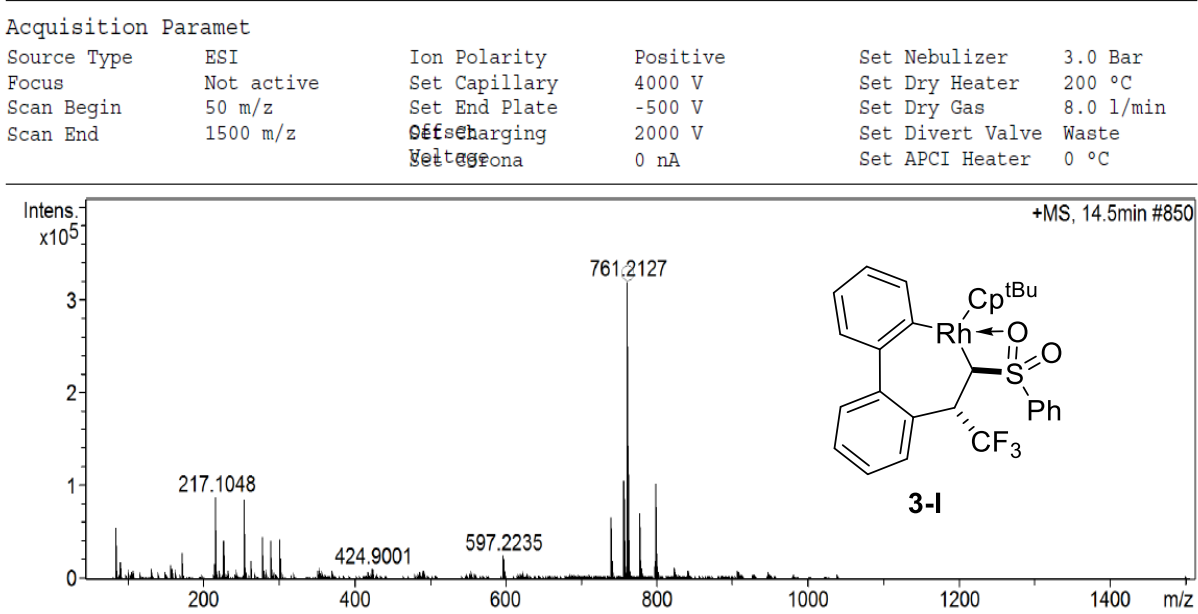

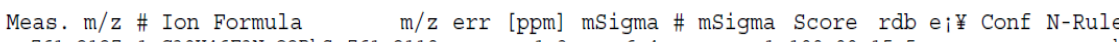

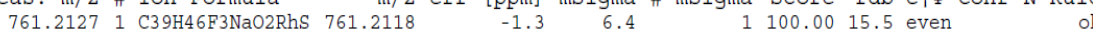

